\title{
Encapsulation of agrichemicals by renewable cellulosic derivatives
}

By

Harrison Monk

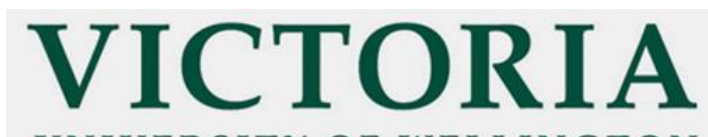

UNIVERSITY OF WELLINGTON

TE WHARE WĀNANGA

O TE ŪPOKO O TE IKA A MĀUI

A thesis submitted to Victoria University of Wellington in partial fulfilment of the requirements for the degree of Master in Science (chemistry)

Victoria University of Wellington 


\section{Abstract}

Cellulose derivatives, charged with fungicides, have been prepared as particles for use as a wood preservative. The particles were designed to encapsulate the current industry-standard chemical agents used to minimise wood degrading fungal action and to deter termites.

A detailed study on the most effective methodology that would be suitable for scaled-up production was undertaken. The methods explored included: double emulsions, solvent diffusion by dialysis membrane and phase inversion emulsification. Particles formed by these methods were characterised by scanning electron microscopy, dynamic light scattering, nuclear magnetic resonance spectroscopy and infrared spectroscopy. The fungicide incorporation was confirmed by nuclear magnetic resonance studies and gas-chromatography analytical analysis. The phase inversion emulsion process was found to be highly effective and readily manipulated to modify particle formation. Particles were successfully prepared containing fungicides in a yield of $35-75 \%$ (method dependant), containing the biocide at approximately $50 \%$ mass of biocide to total particle mass. Thus, this process was optimised through modifying the addition time of the aqueous phase, as well as variation of the surfactant and salt concentrations. With an optimised particle forming method, three fungicides were incorporated into the formulation and subsequently analysed to demonstrate successful biocide incorporation. The biocide charged mesoparticles underwent testing for antifungal action by our industrial partners Lonza. 


\section{Acknowledgments}

I would like to acknowledge my supervisor Dr Simon Hinkley, without whom I would not have gotten here. I would also like to acknowledge to entire Ferrier Research Institute team, in particular the carbohydrates analysis team, with a special thank you to Dr Alison Daines and Dr Norman Avelino for their assistance in various aspects of the project. I would also like to acknowledge Dr Sarah Spencer from Robinson Research Institute, for all her assistance with the scanning electron microscopy work in this project; her knowledge was invaluable. I would also like to acknowledge Dr David Clarke from Callaghan Innovation for his assistance in the use of the Mastersizer 3000, which was responsible for all the particle sizing carried out in this project.

I would also like to acknowledge industrial partners Lonza, in particular Dr Chris Molloy and Stephen Millward for their invaluable input both with supplying necessary chemicals and carrying out the biocide testing on the fungicide loaded particles.

Finally, a big thank you to my partner Rachael Basire for all the help in supporting me both with the thesis and financially; also thank you to my mum for her help in proofreading and financial support.

\section{Declaration and Copyright}

The copyright of this thesis resides with the author. No quotation from it should be published without his prior consent, and information derived from it should be acknowledged.

The work in this thesis was completed by the author at the Ferrier Research Institute, Victoria University of Wellington, New Zealand. It has not been submitted in part, or in whole, for any higher degree at this or any other University. 


\section{List of abbreviations}

SEM scanning electron microscopy

IR infrared spectroscopy

CA cellulose acetate

DS degree of substitution

NMR nuclear magnetic resonance spectroscopy

CAB cellulose acetate butyrate

EC ethyl cellulose

W/O water in oil

O/W oil in water

DLS dynamic light scattering

DSC dynamic scanning calorimetry

PVA polyvinyl acetate

DMA dimethylacetamide

GC-FID gas chromatography with flame ionisation detector

IPDI isophorone diisocyanate

DI Deionised

ACN Acetonitrile

IPDI Isophorone diisocyanate

DABCO 1,4-diazabicyclo[2.2.2]octane

DMSO Dimethylsulfoxide 


\section{Contents}

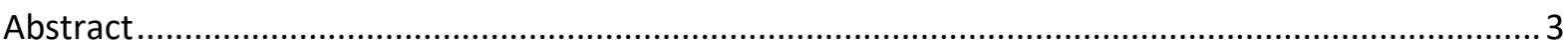

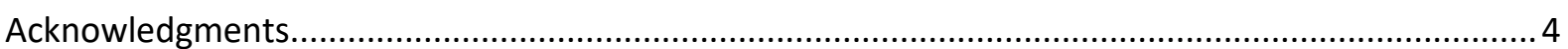

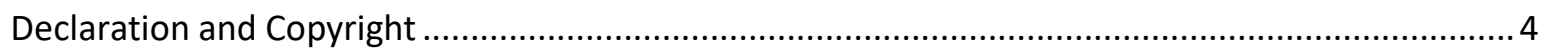

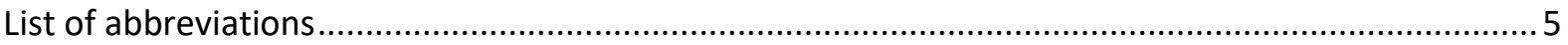

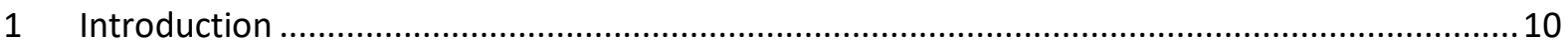

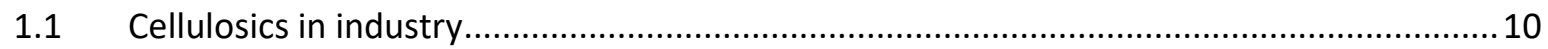

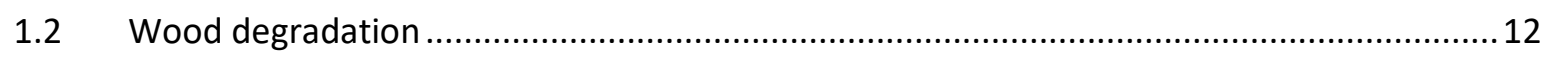

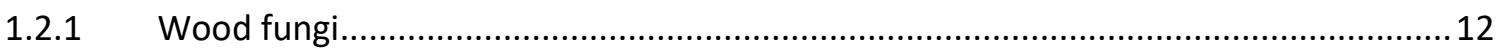

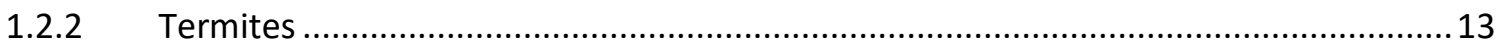

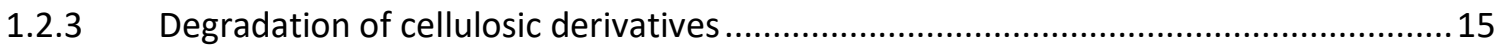

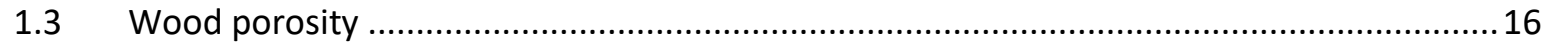

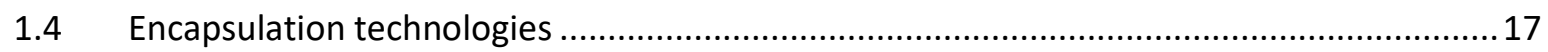

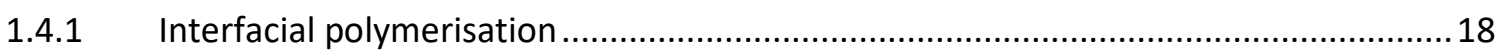

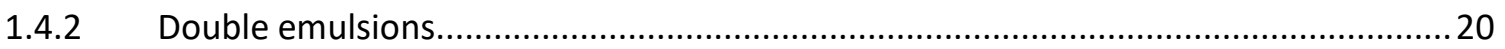

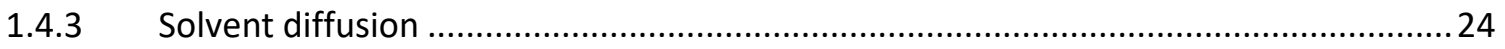

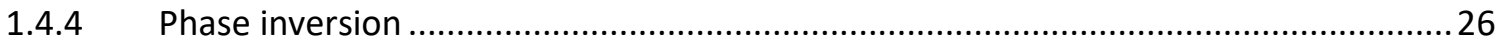

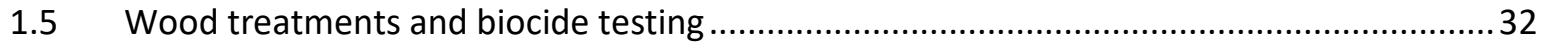

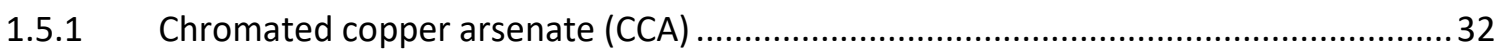

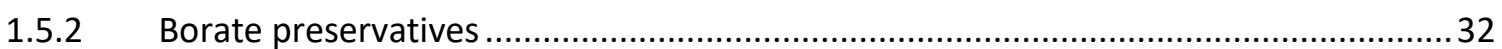

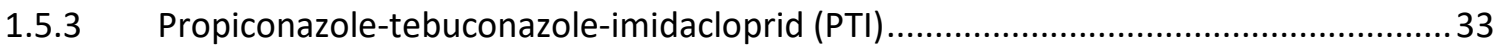

1.5.4 Assessment of wood preservatives in Australasia ......................................................... 33

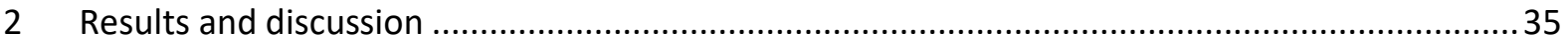

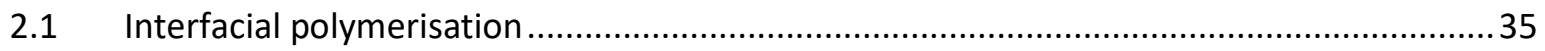

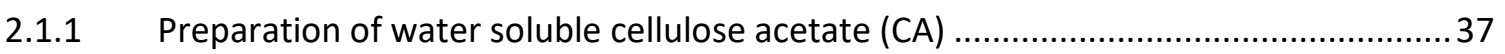

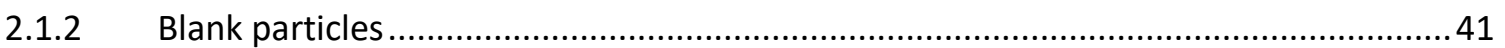

2.1.3 Incorporation of carbohydrate polymers ............................................................... 42 
2.2 Double emulsions

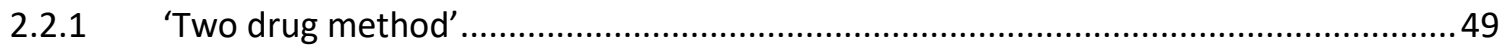

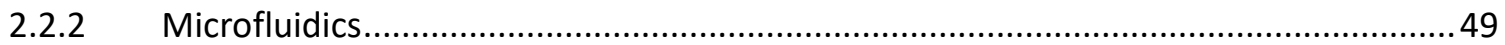

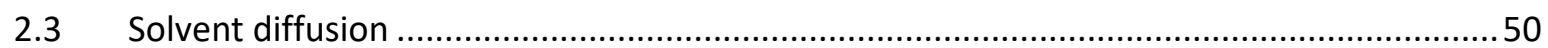

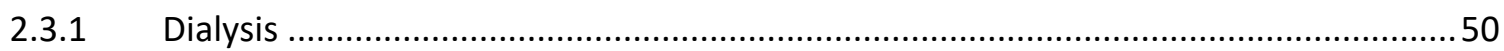

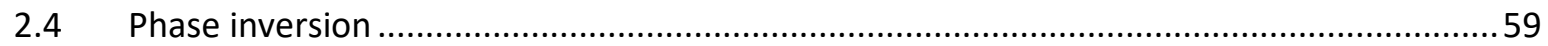

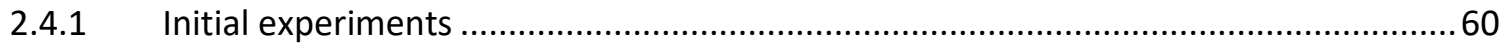

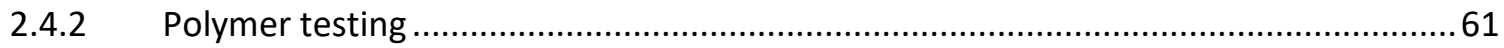

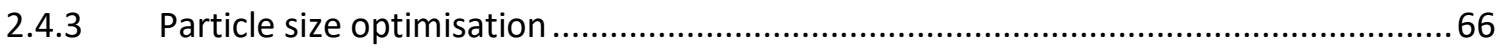

2.4.4 Model compound incorporation..................................................................... 72

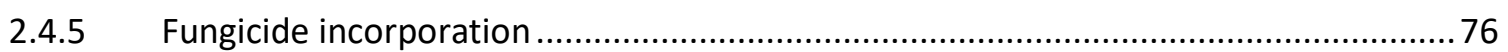

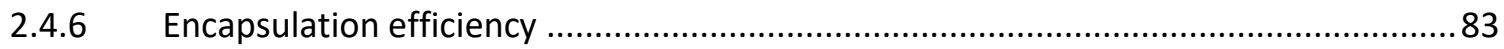

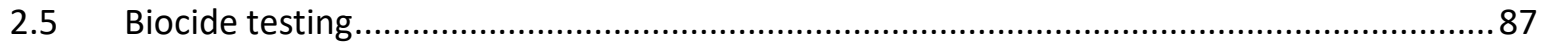

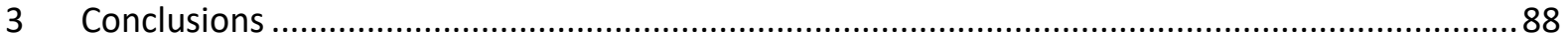

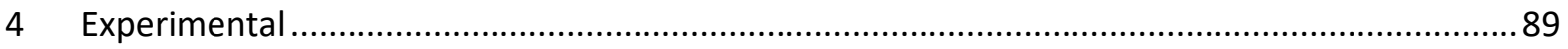

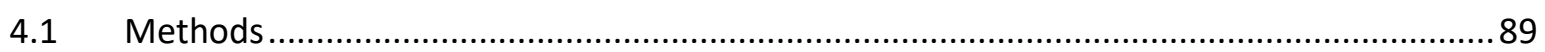

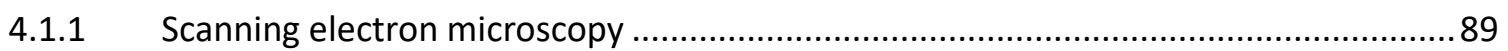

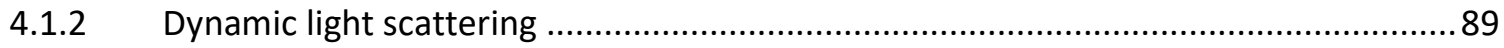

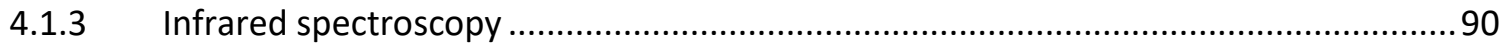

4.1.4 Nuclear magnetic resonance spectroscopy ..................................................90

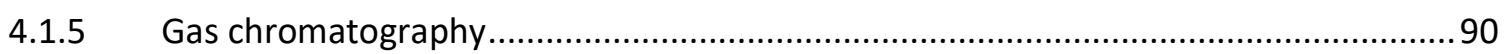

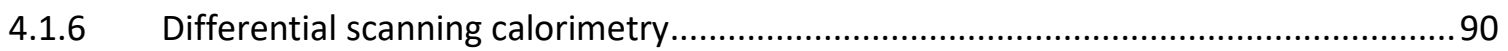

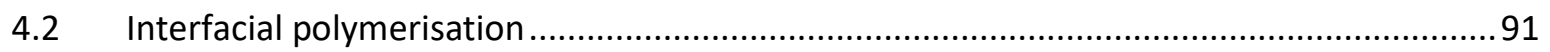

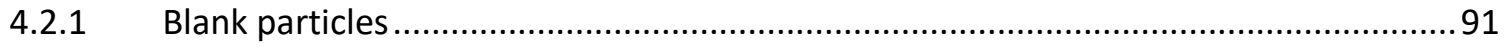

4.2.2 Particles with cellulose derivative.......................................................... 91

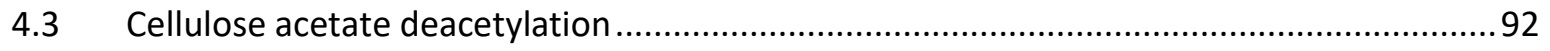

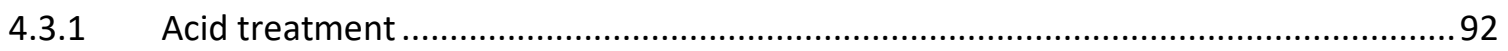

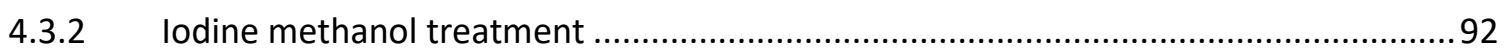




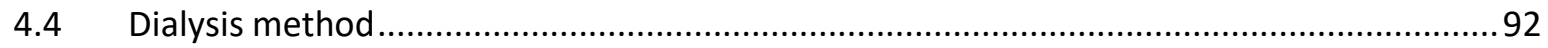

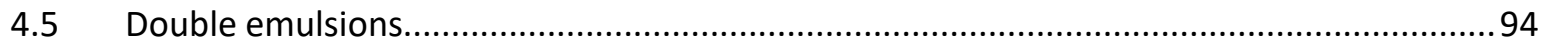

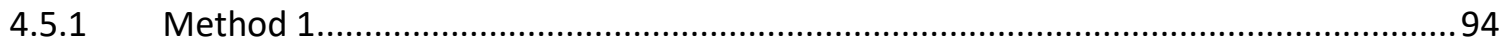

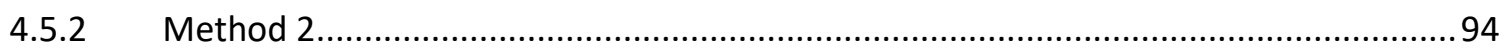

4.6 'Ouzo effect'

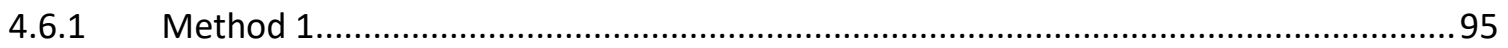

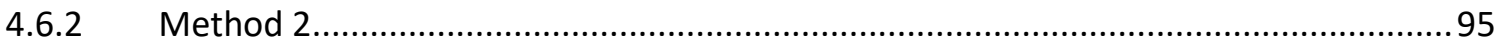

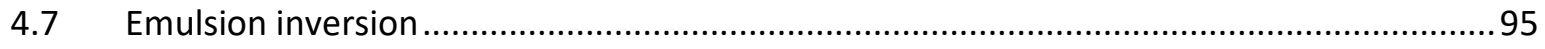

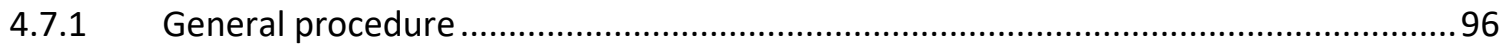

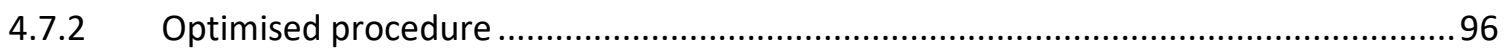

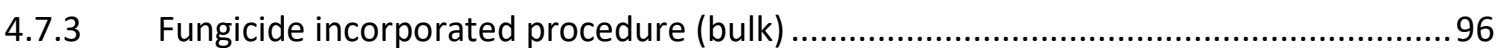

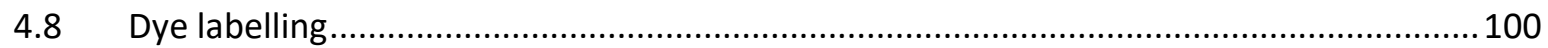

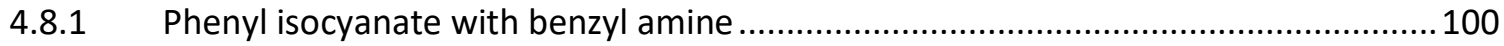

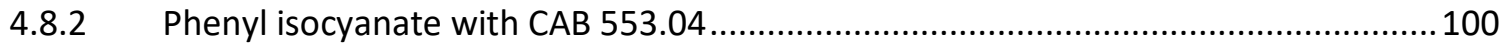

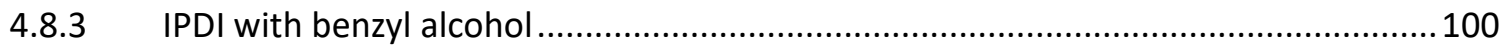

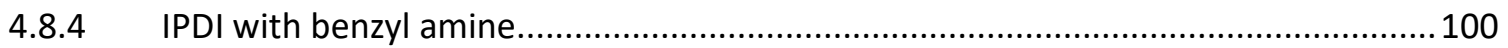

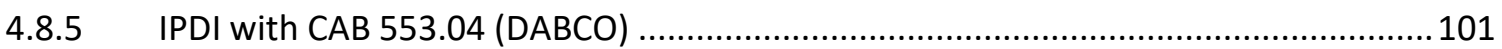

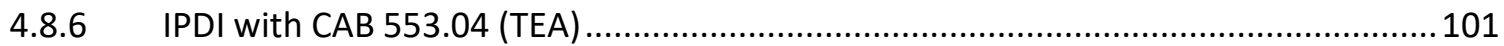

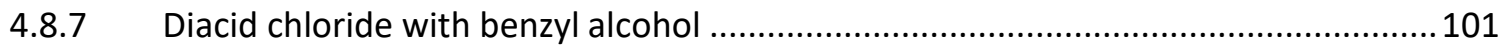

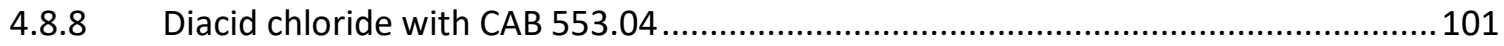

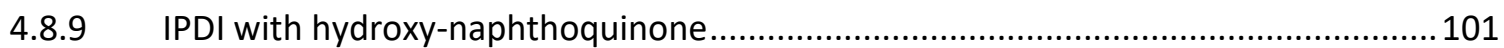

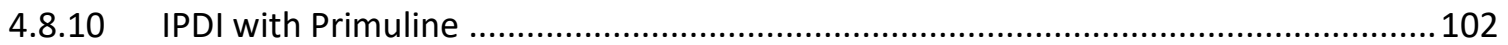

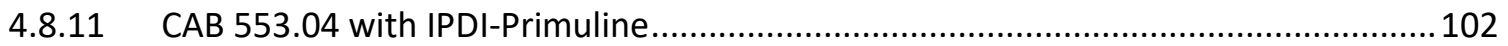

4.8.12 CAB 553.04 with IPDI- hydroxyl-naphthoquinone …............................................. 102

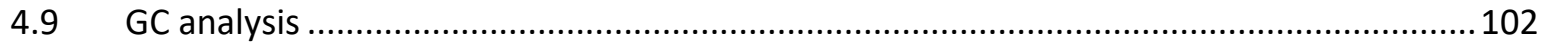

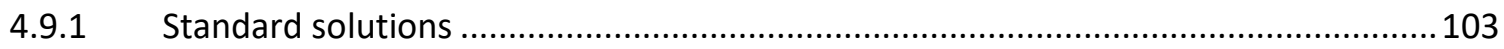

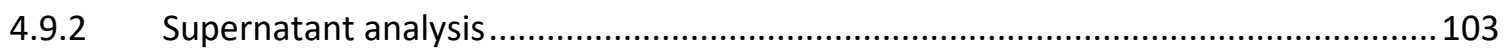

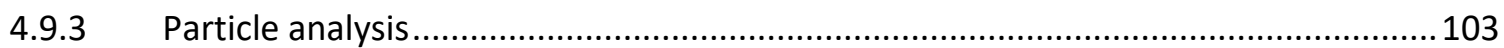




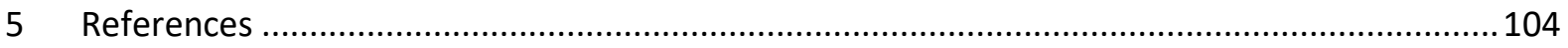

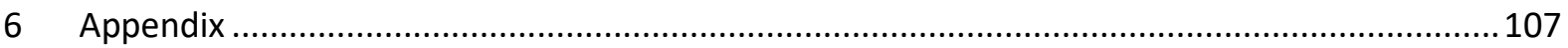

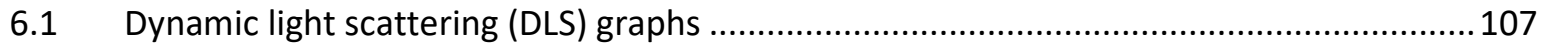

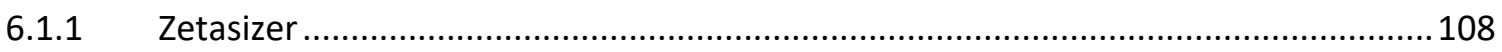

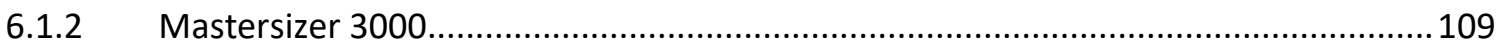

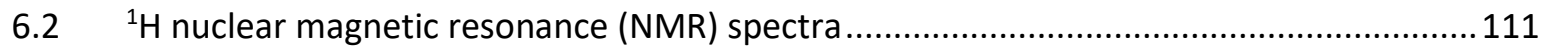

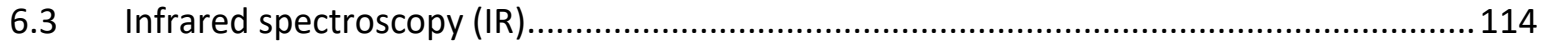

6.4 Dynamic scanning calorimetry (DSC) graphs ............................................................ 117 


\section{Introduction}

\subsection{Cellulosics in industry}

Cellulose is one of the most abundant natural polymers and is a vital component in almost all plants as a structural material. Cellulose was first isolated by French chemist Payen in $1842 .{ }^{1}$ The cellulose structure, shown in Figure 1 consists of a linear polymer composed of anhydroglucopyranose units, which are connected by $\beta(1-4)$-glycoside linkages. The strength of cellulose comes from the intermolecular $\mathrm{H}$-bonding interactions of the discrete polymer chains.

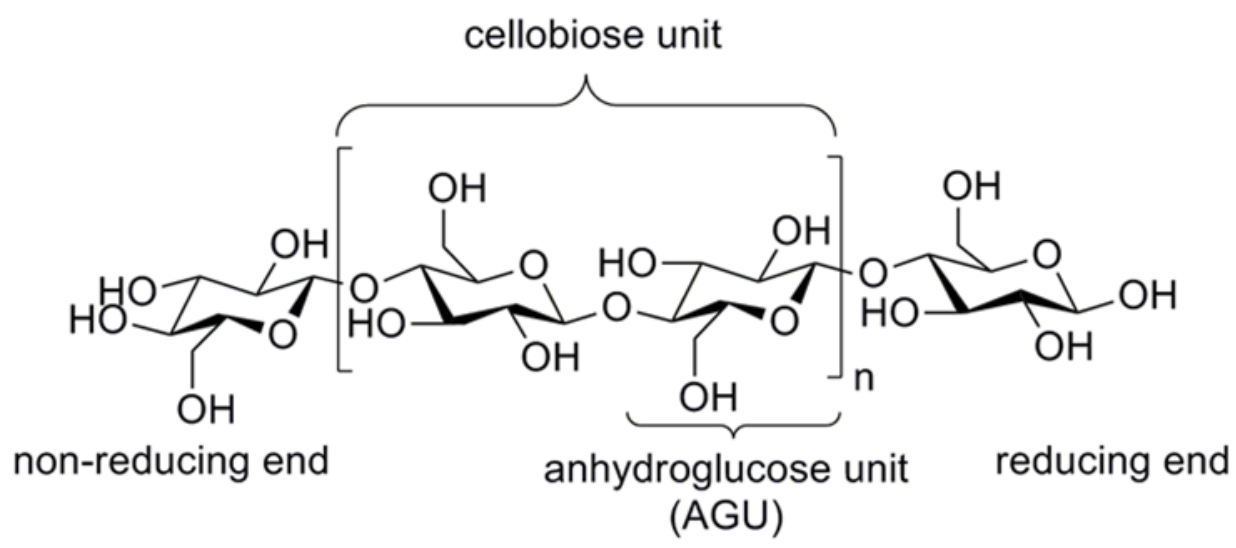

Figure 1: Structure of cellulose

In industry the main use of pure cellulose is in paper manufacture and involves harsh processing of wood into wood pulp using what is known as the Kraft process. ${ }^{2}$ This method uses a mixture of sodium hydroxide and sodium sulfate to break down the wood into wood pulp and separate the different components. To solubilise pure cellulose harsh reagents such as lithium chloride and/or concentrated acids are required, thus its usefulness in industrial applications is limited. To easily solubilise cellulose, the structure must either be chemically modified, or a specialist solvent can be used.

Rayon $^{3}$ is an industrial product that uses pure regenerated cellulose and it was developed as a competitor product to cotton. Rayon is mostly generated from cotton linters which are $95 \% \alpha-$ cellulose, ${ }^{4}$ and this highly purified cellulose is made soluble through modification as the xanthate ${ }^{5}$ or in the more recent methodology using $\mathrm{N}$-morpholine-N-oxide (NMNO) to generate a soluble form that can be precipitated into fibres. However, apart from the aforementioned example, pure cellulose has limited uses in industry because of its insolubility in almost every solvent.

Cellulose derivatives, however, are used extensively as industrial products and commodity materials (Table 1). Cellulose derivatives have different functional groups on the free hydroxyl groups and this changes the structural and electronic properties of the polymer. One of the first derivatives prepared 
commercially was cellulose nitrate, first made in 1832 by Henri Braconnot. The product was formed by the reaction of cellulose (as cotton) with nitric acid. This nitrogenous cellulose derivative's first application was in warfare, where it was used as 'gun cotton' given its high explosive tendency under applied pressure. ${ }^{6}$ Another application of nitrocellulose is its use in film in the late 1800 s, as a flexible film base. Some of the more industrially important cellulose derivatives are the cellulose acetates. These polymers have acetate groups in place of the hydroxyl groups on the glucose monomers of cellulose. They are generated by the reaction of acetic anhydride and acetic acid with pure cellulose in the presence of sulfuric acid. A subsequent controlled hydrolysis reaction is used to cleave sulfate and some of the acetates to achieve the desired properties. The number of acetate groups per monomer is described as the degree of substitution or DS. ${ }^{7}$ The different DS cellulose acetates will have different physical proprieties, for example solubility, glass transition temperature and hardness. These different properties arise from the different steric and electronic effects of the varying number average of acetate groups on the cellulose polymer. Due to the wide variety of different properties that can be tuned into the polymer they have many uses in various industrial and scientific applications.

Table 1: Applications of different cellulose derivatives

\begin{tabular}{lll}
\hline Cellulose derivative & Application & Reference \\
\hline Nitro cellulose & Film base & 6 \\
\hline Cellulose acetate & Gun cotton & \\
& Film base & 8 \\
& Cigarette filter tips & 9 \\
\hline Cellulose acetate butyrate & Filter membranes & 10 \\
& Drug delivery & 11 \\
\hline Ethyl cellulose & Nanocomposites & 12 \\
\hline Methyl cellulose & Food additives & 13 \\
\hline
\end{tabular}

Other cellulose derivatives used by industry are methyl cellulose and ethyl cellulose. These two derivatives have similar properties and thus similar uses in industry. Methyl celluloses are primarily used as viscosity modifiers in common cosmetic products such as hair shampoos, liquid soaps and toothpastes. This is due to their inert nature along with their ability to gel at low concentrations. Ethyl cellulose is used as a food thickener, however it has also been used in applications of drug encapsulation. ${ }^{15-16}$ The general structure of these cellulose derivatives is demonstrated in Figure 2, 
where the R groups are either alkyl or acetyl groups. Other cellulose derivatives of note are the cellulose acetate butyrates, which have a combination of hydroxyl, acetate and butyrate groups bound to the cellulose monomers. These cellulose derivatives are primarily used in coating applications and pharmaceutical research ${ }^{17}$ where their physical properties give them the advantage over other cellulosics.

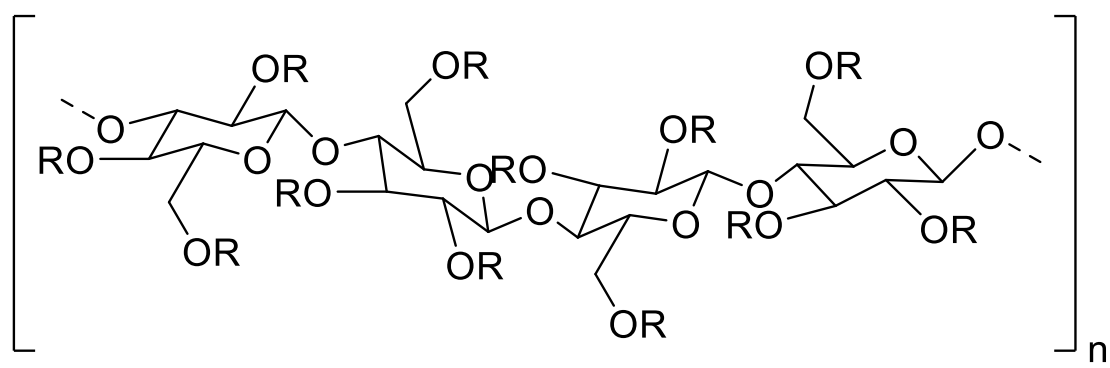

$\mathrm{R}=\mathrm{H}$ or Me or Et or Ac

Figure 2: Cellulose derivative general structure

\subsection{Wood degradation}

Wood degradation due to irreversible parasitic damage is an issue that is commonly dealt with by crude methodologies which have not changed for many decades. It is caused primarily by two different pests which differ in their method of cellulose digestion; termites and wood fungi. Termites will physically eat the wood and break down the cellulose through digestion, whereas wood fungi release cellulase enzymes to break down the wood. This project aims to produce a scale suitable method for the production of cellulosic particles which will encapsulate a fungicide and/or insecticide. Thus, the process by which wood fungi and termites degrade wood is a key factor to be considered, as degradation of the particle shell by these pests is a critical step in the success of the particles as a preventative formulation.

\subsubsection{Wood fungi}

Wood fungi are separated into three different groups; brown rot, white rot and soft rot. The different colours of rot signify the different subspecies, each of which have distinct preferred environmental conditions and enzyme types. ${ }^{18}$ Brown rot fungi typically degrades cellulose through both noncatalytic and catalytic methods. Lignin is normally not broken down by brown rot fungi and as a result, the degradation normally leaves a brown crumbly lignin-rich residue. White rot fungi on the other hand are typically found to degrade hardwoods and use both cellulosic and lignin enzymes to break down the wood. The damage caused by white rot can be greater than brown rot due to their ability to degrade all the materials in wood. Soft rot fungi are typically found to attack high moisture and low lignin woods. As these woods are less used in buildings, preventing the manifestation of this type of 
fungi is not as important. A recent study ${ }^{19}$ into the degradation patterns of two white rot fungi, Pleurotus ostreatus and Trametes versicolor, was able to show that they both exhibit similar degradation pathways. Scanning electron micrographs of the degradation of wood are shown in Figure 3.

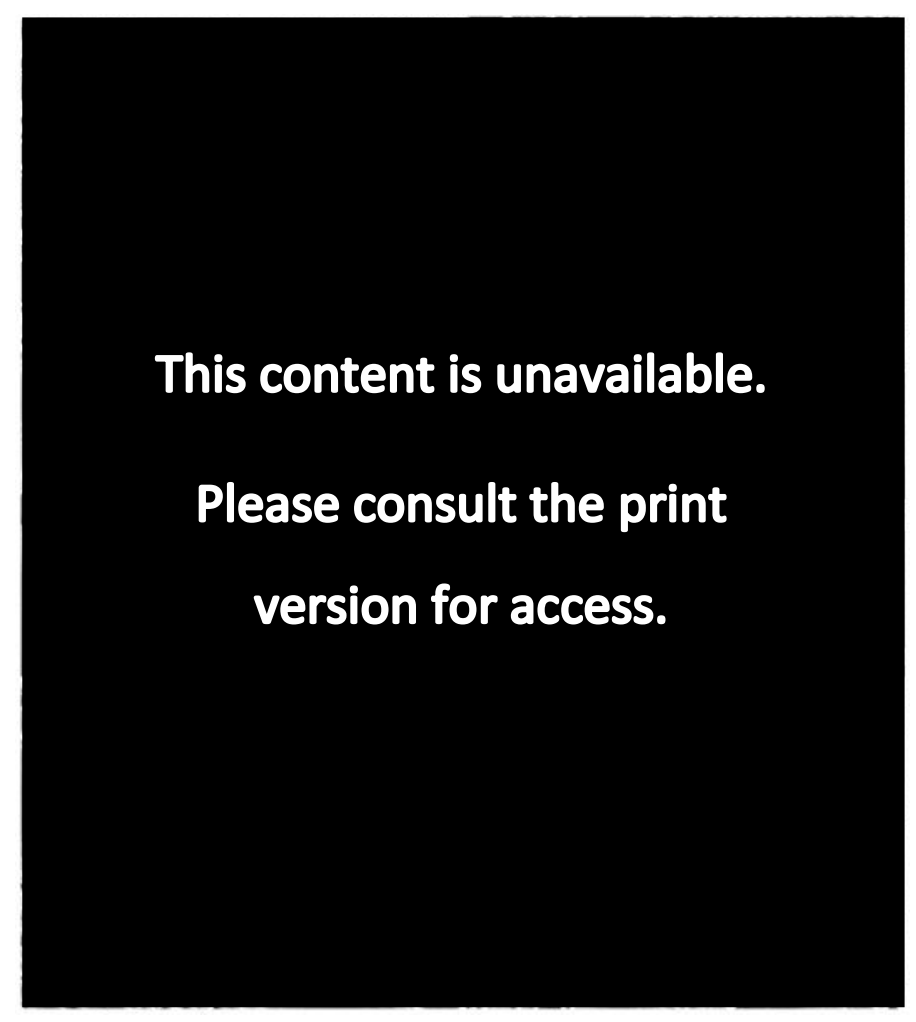

Figure 3: SEM images of white rot fungal degradation of wood, reproduced from ${ }^{19}$

Their data showed a significant percentage of lignin was degraded when compared to cellulose after a 120-day exposure period to both fungi. This is indicative of a white rot fungus and it can be concluded that white rot fungi pose the largest threat to wood constructed buildings, due to their non-selective degradation of all different materials in wood.

\subsubsection{Termites}

Termites are a wood eating insect closely related to cockroaches. They are found in almost every continent in the world and over 2000 species are currently known. In the developing world, the use of renewable building materials like wood can be threatened by degradation caused by termites. The degradation of wood by termites is substantial and the cost of termite related damage was estimated in the south-western United States to be approximately $\$ 1.5$ billion each year. ${ }^{20}$ Their digestion of cellulose is said to be mediated by gut microbiota that allow the full breakdown of cellulose and lignin in wood. A recent article ${ }^{21}$ on the microbiota in the hindgut of higher termites used metagenomic analysis of the bacterial diversity in the hindgut and indicated that previously unknown cellulose 
degrading organisms were present. Figure 4 shows the breakdown process of cellulose in the hindgut of the termite.

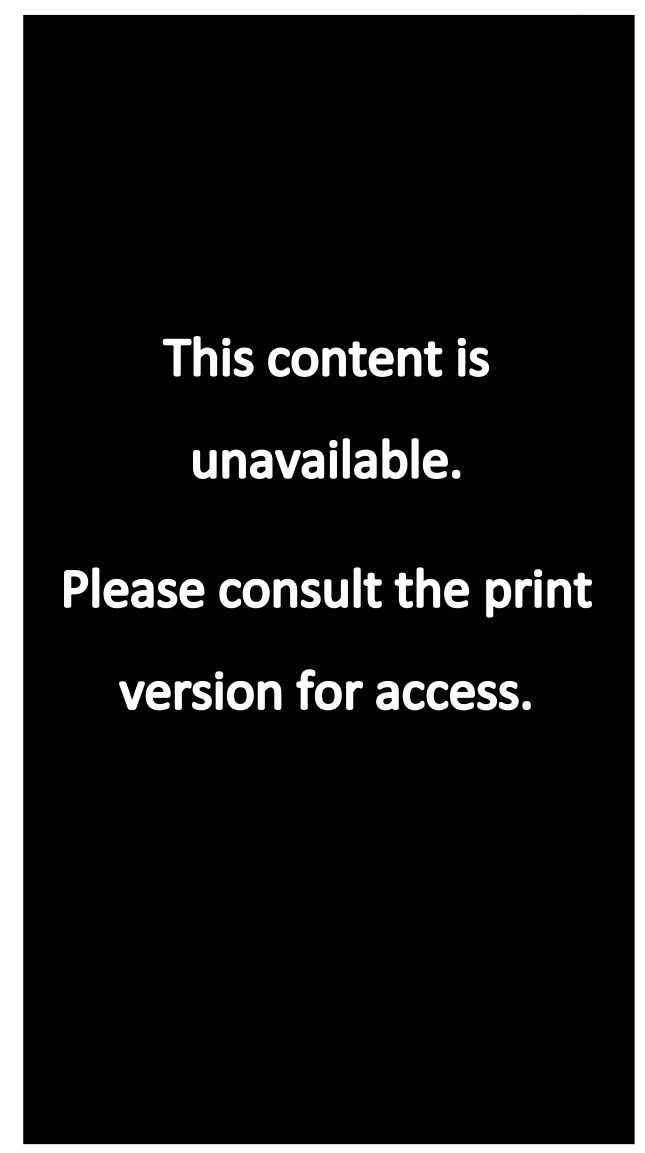

Figure 4: Breakdown process of cellulose in termite hindgut, reproduced from ${ }^{22}$

The diagram shows that the breakdown of cellulose is carried out by various enzymes, starting with the hydrolysis of the cellulose, leading to depolymerisation. Next fermentation of the oligomers form intermediates that are then further degraded to acetate, which is then reabsorbed by the termites. ${ }^{22}$ The diversity of the microbiota in termites is large and varies from species to species and lower to higher termites (Figure 5). 


\section{This content is unavailable.}

\section{Please consult the print}

\section{version for access.}

Figure 5: Comparison of microbiota levels in various species and types of termites, reproduced from ${ }^{22}$

\subsubsection{Degradation of cellulosic derivatives}

The work described herein proposes to generate cellulose-derived particles to encapsulate agrichemicals. Thus, an important aspect of this project is the ability of the fungi to degrade the cellulose derivatives. Early studies on cellulose derivative degradation showed varying results; some found that the level of degradation was less than that of pure cellulose ${ }^{23}$ others that there was no significant degradation occurring. ${ }^{24}$ However, recent studies on the degradation of cellulosic derivatives by various natural entities showed that some fungi do have enzymes that can degrade modified celluloses. ${ }^{25}$ The level of degradation is determined by several factors, including the degree of substitution, distribution of substitution, and the specific structure of the enzyme. An example of an enzyme known to degrade cellulose derivatives is shown in Figure 6.

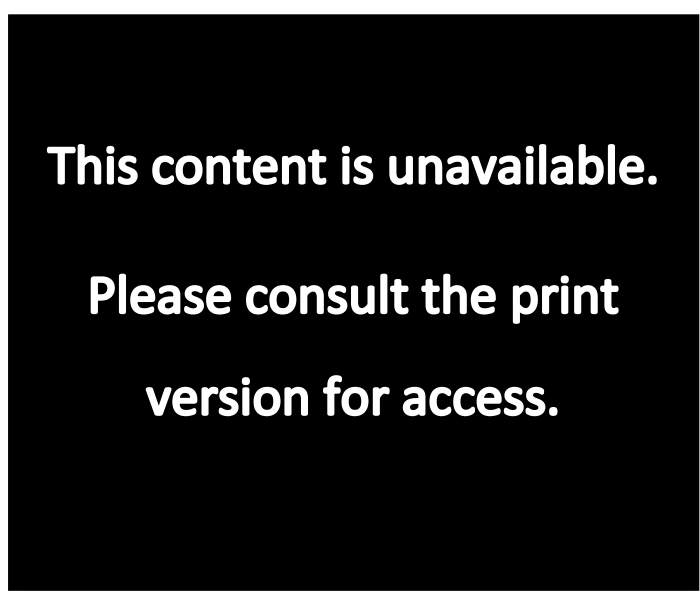

Figure 6: Known cellulase enzyme, endoglucanase Cel7B, reproduced from ${ }^{25}$ 
The enzyme above (Figure 6) was isolated from the fibrous fungi Trichoderma reesei. This fungi was demonstrated to have high cellulase and hemicellulase activity by Ooshima and co-workers. ${ }^{26}$ They discovered that cellulose derivatives with a lower degree of substitution had higher degradation rates. Thus, some of the fungal cellulases can degrade some cellulose derivatives. A key question is whether this organism and associated enzymes can break down the capsule structure to release the active agent. Further research of the degradation of cellulose derivatives show acetyl esterase enzymes to be of importance in this process. ${ }^{27}$ In this work they came to a very similar conclusion as the aforementioned work, that the lower substituted cellulose derivatives had better degradation profiles than higher substituted ones. This correlates well with the most recent work in this area, indicating that the DS of a cellulose derivative is the key component which controls how well it can be digested by cellulose degrading enzymes. ${ }^{26}$

\subsection{Wood porosity}

Wood has an extensive network of micro-pores that extends from its surface. These pores are vital for the plant to get water and nutrients. There are three primary pore sizes found in processed wood; micro-, macro- and meso-pores (see figure 7). Common tree types have different distributions of these pore sizes, and these distributions dictate their pore densities. The importance of pore density is that it determines properties of wood such as flow, adsorption and impregnability. ${ }^{28}$ These parameters are important to understand for this project as the primary objective is to impregnate wood pores with cellulosic encapsulated agrichemicals. The diagram shown in Figure 7 shows the distributions of these pore sizes in various woods.

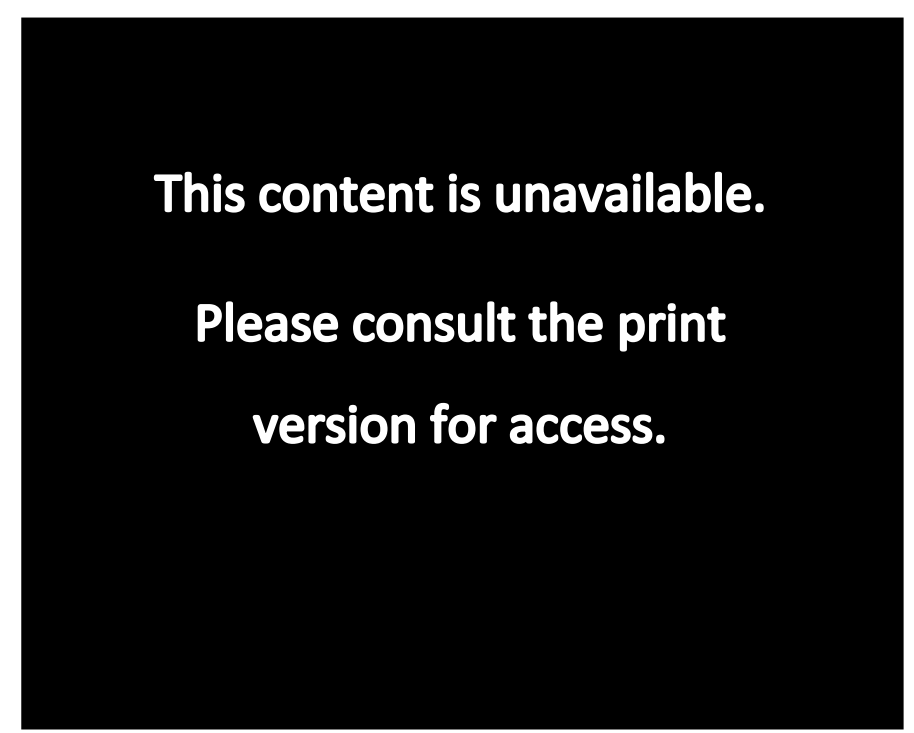

Figure 7: Distributions of pore sizes in different wood types, reproduced from ${ }^{28}$ 
The diagram above shows the wide variety of pore sizes in the three wood types presented. Of most importance are the wood types commonly used in building construction, normally hardwoods, due to their inherent strength. The pore sizes prominent in these woods are the micro- and macro- pore sizes, which gives a desired particle size range of approximately $80-500 \mathrm{~nm}$.

\subsection{Encapsulation technologies}

Encapsulation is a technique that has been used for many decades, particularly in the food and cosmetic industries. ${ }^{29-30}$ Encapsulation is used primarily to protect active compounds from the surrounding environment in which the system was designed to operate, such as high or low $\mathrm{pH}$ and temperature changes. Another reason to utilise encapsulation would be for slow release treatments, primarily used in drug delivery systems. These systems can vary from liposomes ${ }^{31}$ to emulsion formed hollow particles. ${ }^{32}$ The use of emulsion technologies for encapsulation is one of the most common techniques used and is used in a variety of applications.

An emulsion is where an oil or organic phase is mixed under high sheer with a water or aqueous phase and generates droplets due to the surface interactions of the two phases. This is shown diagrammatically in the following Figure 8.

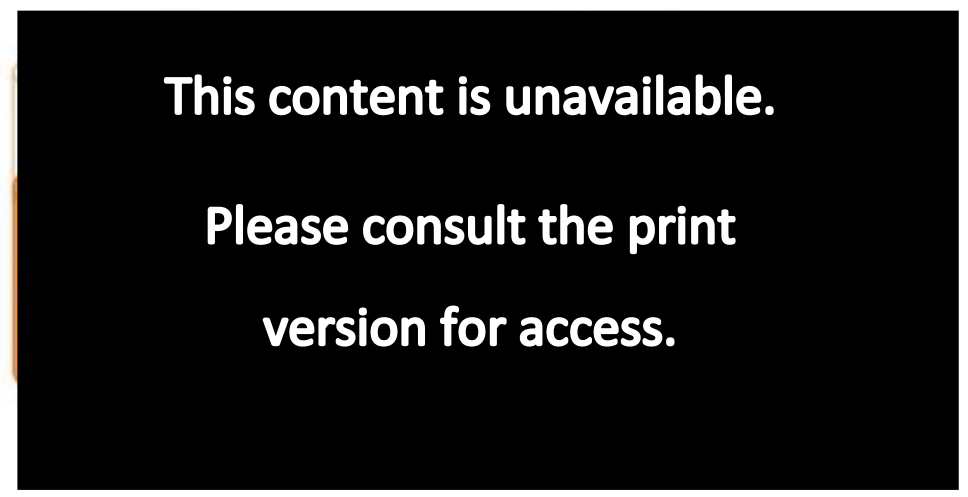

Figure 8: Diagram of a simple emulsion, right is $\mathrm{O} / \mathrm{W}$, left is $\mathrm{W} / \mathrm{O}$, reproduced from ${ }^{33}$

Whichever phase is added under sheer will determine which are the external and internal phases of the droplet. The figure above shows an oil in water emulsion $(\mathrm{O} / \mathrm{W})$ in the right image and water in oil emulsion (W/O) in the left image. Droplets will form within the external phase and if a polymer is dissolved into the internal phase, removal of the internal phase (i.e. it is volatile and removed by evaporation) will cause the polymer to precipitate and form capsules. The control of the emulsion properties is determined using surface active agents that will modify the surface energy of the oil/water interface, allowing for the formation of a stable emulsion. 
The encapsulating matrix to be permeable to the active compound, however; to the extent they release the active agent at a desired rate. Examples of such an application are demonstrated by utilisation of cellulose acetate butyrate to encapsulate the water-soluble drug Tetracycline $\mathrm{HCl}$ for a self-propelled drug release system. ${ }^{10}$ The capsules were produced by using an $\mathrm{O} / \mathrm{W}$ emulsion and were hardened by solvent evaporation to precipitate the cellulose. This methodology has many applications for cellulose based encapsulation processes for drug delivery systems. The capsules produced by this method are shown in Figure 9. The capsules can have several spheres inside the primary capsule, which is the result of mini-emulsified particles within the main droplet.

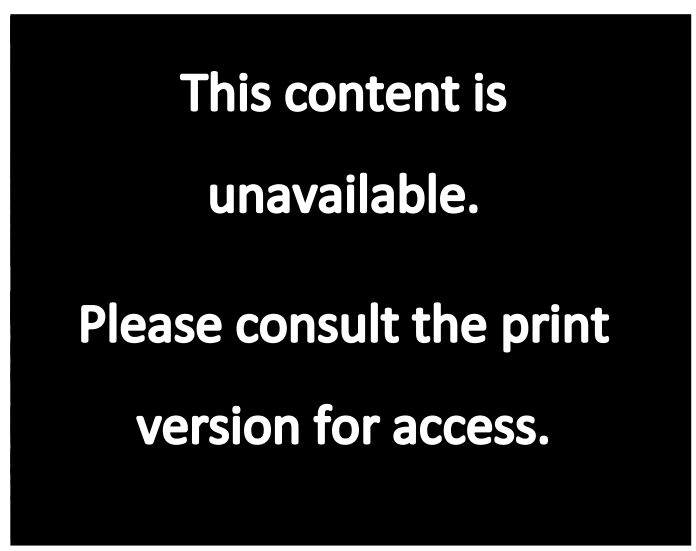

Figure 9: Cellulose acetate butyrate capsules, reproduced from ${ }^{10}$

\subsubsection{Interfacial polymerisation}

Interfacial polymerisation is a process used to produce hard polymeric particles by the polymerisation of two monomers at the phase interface of an emulsion. The most common reaction used for this process is a polycondensation reaction between two reactive monomers, where the reaction is localised at the phase interface between two immiscible phases. The process was first developed by Emerson Wittbecker in $1959^{34}$ who used a diacid chloride and a diamine as the monomers for the polycondensation reaction. There are a range of monomers that can be used in this process that will generate different polymers and consequently allows the properties of the polymers to be tuned to suit the application. Interfacial polymerisation is used widely in industry due to the ease with which the process can be scaled up, along with the range of particles that can be made.

A recent review ${ }^{35}$ on interfacial polymerisation methods and compounds used shows this variety in substrates and the resulting polymer that are produced. Polyurethanes and polyureas are amongst the most common polymers produced by this method to form microparticles. These are used because of their inherent resistance to surrounding chemical environments ${ }^{35}$ and the ease with which they form hollow capsules containing a liquid core. Therefore, they are good candidates for application in drug delivery, where the capsule wall thickness will determine the rate at which the active compound 
is released. Polyurethanes are formed from the condensation of a diisocyanate with a diol, whereas polyureas are formed by the reaction of a diioscyanate and a diamine. Due to the higher nucleophilic properties of an amine group verses a hydroxyl group, the polyureas form with much higher rates. Another advantage for polyurea based microparticles is the reactive hydrogens that urea groups contain, which can be sites for cross-linking between polymers, which then further changes the properties of the material. Cross-linking can lead to stronger, more chemically resistant particles, which is a quality highly sought after by industry. A general reaction scheme for the formation of polyureas is shown in Figure 10.

$$
\mathrm{OCN}^{-\mathrm{R}_{1}} \mathrm{NCO}+\mathrm{H}_{2} \mathrm{~N}^{-\mathrm{R}_{2}} \mathrm{NH}_{2} \longrightarrow \mathrm{OCN}^{-\mathrm{R}_{1}} \underset{\mathrm{H}}{\mathrm{H}} \mathrm{N}^{-\mathrm{R}_{2}} \mathrm{NH}_{2}
$$

Figure 10: General reaction scheme for a diisocyante with a diamine

The process for the formation of hollow particles using this method is shown diagrammatically in Figure 11.

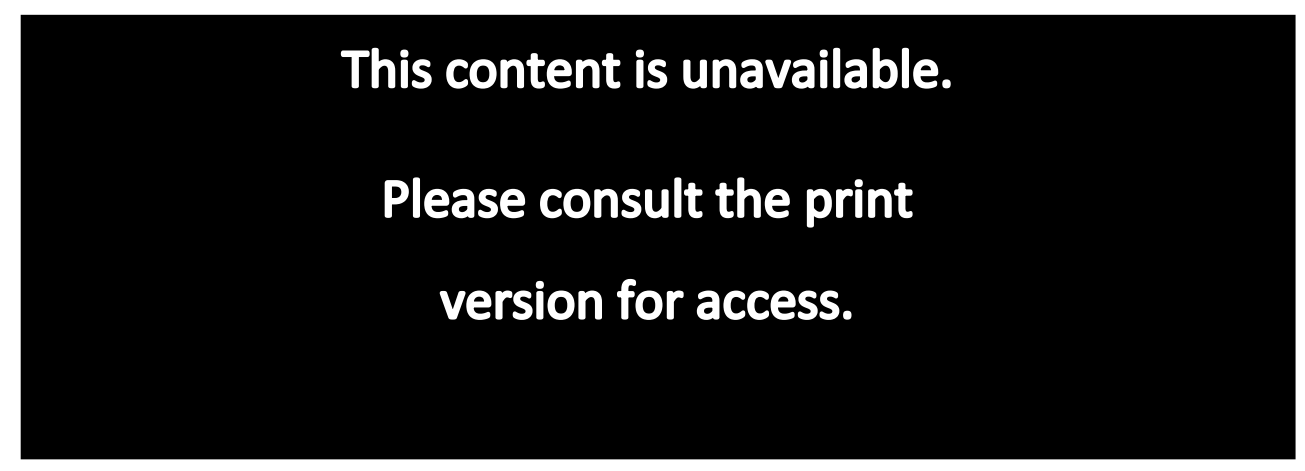

Figure 11: General interfacial polymerisation flow diagram, reproduced from ${ }^{36}$

The diagram above shows the oil phase will have the active compound dissolved in it along with monomer 1 , which will be the diisocyanate as they are normally organic soluble. An emulsion is then formed using this organic phase with an aqueous phase containing surfactant and stabiliser compounds. Monomer 2 is added after the initial oil in water (O/W) emulsion is formed, which will be the diamine as this is water soluble. Both monomers will meet at the phase interface and will react with each other once is heat applied to the system. This will form the polyurea polymer at the outside of the droplets, forming hollow particles, after the evaporation of volatile internal phase, with the active compound encapsulated inside.

An example of this process being used in the application of delivering pesticides was a study by Zeneca Ag Products in Richmond, California. ${ }^{37}$ In this work they encapsulated various pesticide oil formulations using interfacial polymerisation of toluene diisocyante and polymethylene- 
polyphenylisocyanate to form a multifunctional particle surface. They used just these two diisocyanates for the formulation, instead of using a diisocyanate and a diamine to form the polyurea polymer. This is possible because an isocyanate, when reacted with water, will form an amine with the elimination of $\mathrm{CO}_{2}$, and this pre-reaction of the isocyanate to provide the reactive amines that were then polymerised by the remaining isocyanate groups. This formed a mixed polyurea that encapsulated the active compound, thus enabling the polymer to have varied properties throughout its cross-section.

They demonstrated the wall of the particles were formed in an asymmetric way due to the water initiating the reaction, and because of its unavailability in the organic phase the polymer forms differently over the course of the reaction time. This was found to generate a denser outer layer and a less dense inner layer, which lead to limited leaching of the pesticide. This was a highly desirable quality for this work, as they were looking to produce particles that would retain the pesticide inside and only release it once the particle was broken. With the leaching studies that were carried out, they found the more pronounced this polymer shell orientation was, the lower the leaching rate of the pesticide was. This control of the leaching characteristics of particles is an important part of encapsulation technologies for the application in drug delivery. This work demonstrates this aspect of the particles can be tuned to give the desired properties.

\subsubsection{Double emulsions}

Double emulsion techniques have been used for many decades for the generation of hollow particles, and cellulosic polymers have been used widely to form the outer layer. The first in-depth studies into double emulsions were done a few decades ago ${ }^{38}$, however its application for drug delivery has been a more recent development. A double emulsion is where an initial primary emulsion of either water in oil $(\mathrm{W} / \mathrm{O})$ or oil in water $(\mathrm{O} / \mathrm{W})$ is further emulsified in another phase of either water or oil. $\mathrm{A}$ diagrammatic example of a double emulsion is shown in Figure 12.

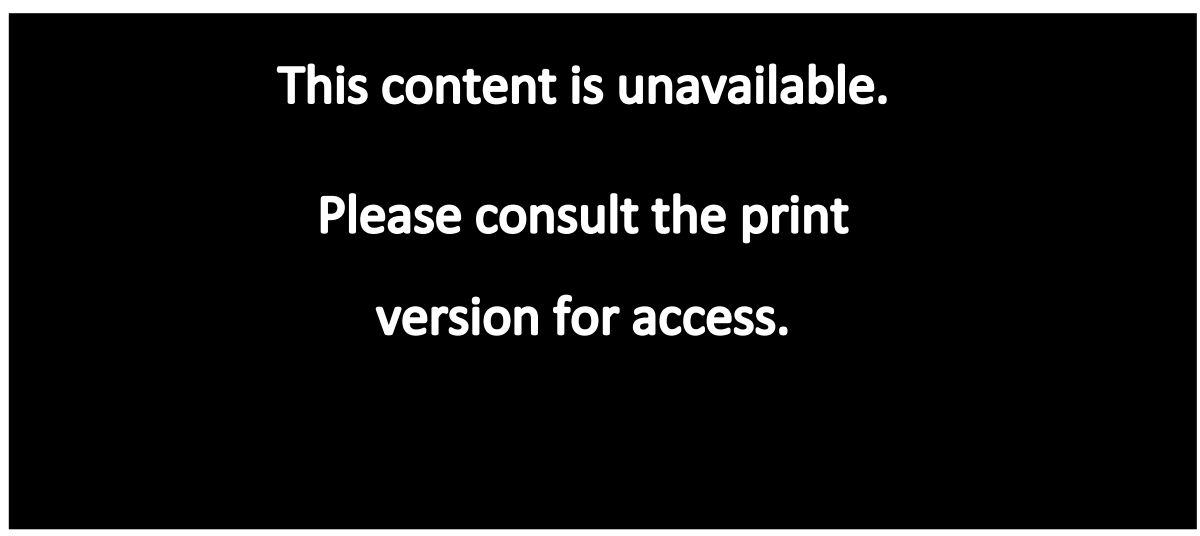

Figure 12: Double emulsion process, reproduced from ${ }^{32}$ 
Double emulsion systems can form as several types; the external phase droplets encapsulate either a single inner phase droplet, several droplets or many. Figure $\mathbf{1 3}$ depicts these three main double emulsion forms.

\title{
This content is unavailable.
}

\section{Please consult the print}

\author{
version for access.
}

Figure 13: Types of double emulsion forms, reproduced from ${ }^{38}$

A single double emulsion form will predominate in any given system over the others, thus combinations of these forms are not found in double emulsion systems. This is primarily because the energetics of a system will prefer one over the others. Type $A$ is the simplest system with a single droplet encapsulated, and is the predominnt type that will be used in this project. The type $\mathrm{C}$ system has shown to be the most effective system for slow release applications. This is due to the reduction in surface tension of the outer droplet and the higher surface tension of the smaller droplets inside, thus generating a driving force for the inner phase to diffuse out. An important factor for generating a double emulsion system is understanding how different surfactants will affect the target system. A recent review ${ }^{39}$ discusses the factors governing surfactant choice in a given double emulsion system. The formation of a double emulsion can be either one-step or two-step; the two-step method was previously discussed where a primary emulsion is further emulsified. The one-step method has been shown to give more stable double emulsions ${ }^{40}$, due to the fact that the two-step method may introduce instability at the second step. Given this, the choice to use a two-step over a one-step process will determine the surfactant choice as well.

Double emulsions have several advantages over normal single emulsions. These include less propensity for the inner phase to diffuse out (for type A systems), and the ability to encapsulate both hydrophilic and hydrophobic active compounds. The ability to encapsulate two active compounds in the one system has many benefits, such as in a recent study ${ }^{41}$ using a double emulsion system of two drugs that had a synergistic effect. These drugs were metformin hydrochloride and glipizide, which are used in the treatment of type II diabetes. They proposed a novel drug delivery system using a $\mathrm{W} / \mathrm{O} / \mathrm{O}$ double emulsion system with ethyl cellulose as the encapsulation polymer. This system was intended as a slow release method, thus porosity in the external phase was necessary. An SEM image of the particles is shown in Figure 14. 


\title{
This content is unavailable.
}

\section{Please consult the print}

\author{
version for access.
}

Figure 14: SEM micrograph of double emulsion particles, reproduced from ${ }^{41}$

\subsubsection{Microfluidics}

Microfluidics encompasses a large variety of different disciplines and areas ${ }^{42}$. However, in general it is the manipulation of fluids on the macroscale, involving their mixing and subsequently generating emulsions. One of the primary forces at play in this area is the use of capillary action. Capillary action is the ability for a fluid to move through tubing that is sufficiently small enough for surface tension to dictate its movement. Figure 15 shows how different surface tensions of fluids affect their capillary action.

\section{This content is unavailable.}

\section{Please consult the print} version for access.

Figure 15: Capillary action of mercury vs water, reproduced from ${ }^{43}$

Water is a polar fluid and has a low surface tension. This means that when interacting with glass, it will cause the meniscus to angle up and the force acting on the fluid in the capillary will be up. The opposite is true for mercury which has its meniscus angling down. Microfluidics manipulate this natural process by adjusting the surface tension of the fluids using surfactants. In our application, only the microfluidic technologies that relate to the production of drug delivery systems will be of interest. 
Microfluidic systems are well known to be used in the generation of double emulsion-like products, and given the ease of use, this system is highly applicable for a scaled-up method for producing hollow particles. A recent review ${ }^{44}$ of the application of microfluidics for drug delivery systems shows the depth of application and interest in this particular field. One of the most important advantages of this process vs double emulsion methods is the ability to tightly control particle size and distribution. Figure 16 shows the various capillary designs that can be used for the encapsulation.

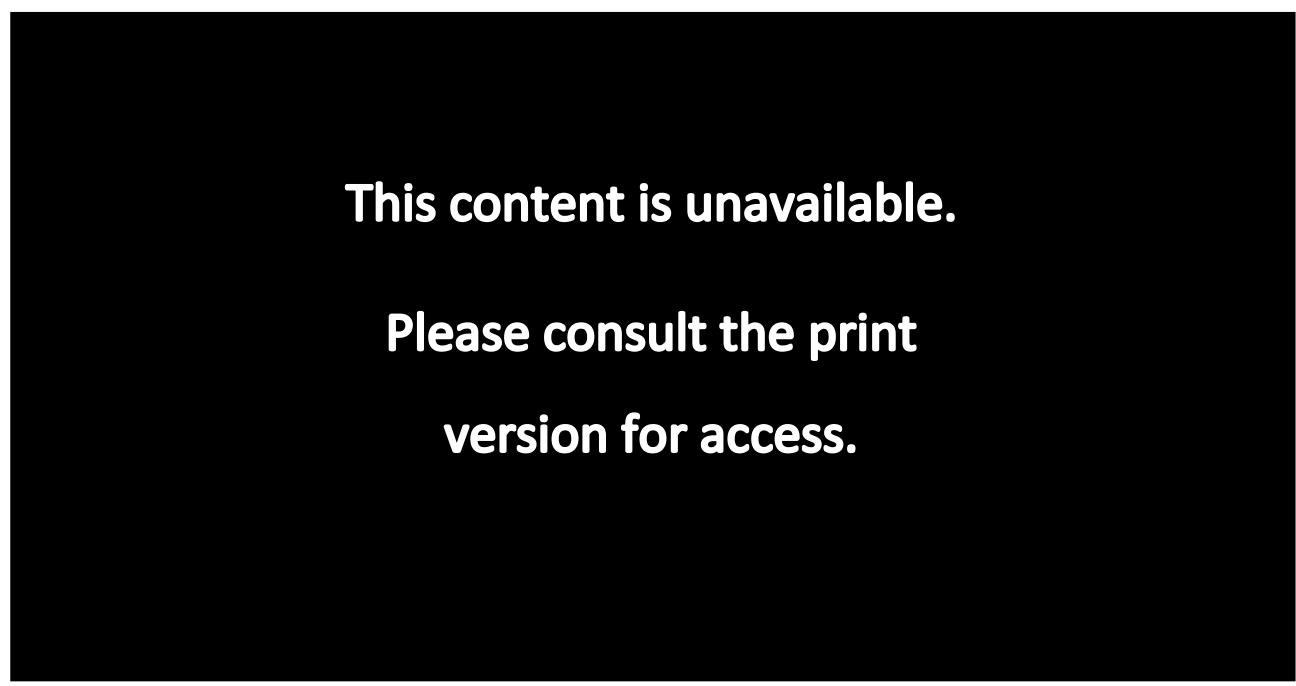

Figure 16: Capillary designs for microfluidic particle producing systems, reproduced from ${ }^{44}$

These different junctions can be applied to give the desired outcome, be it a primary W/O emulsion or a multiphase single droplet. However, the general principle lies in the shearing of the droplets at the exit of the capillary, where the capillary exit diameter and the surface tension of the fluid determines the droplet size. The methods shown in the above diagram are for single or primary emulsion-based systems; to produce a double emulsion with this technique, a two-step process must be used. The double emulsion systems $\mathrm{W} / \mathrm{O} / \mathrm{W}$ and $\mathrm{O} / \mathrm{W} / \mathrm{O}$ are typically generated with this method. The process in general consists of simplifying a two-step flow system utilising two of the capillary designs, either a T-junction or co-flowing. The process can be demonstrated by a previous study ${ }^{45}$ using microfluids with ethyl cellulose for a drug delivery system; Figure 17 shows this diagrammatically. The process shown generates hollow $\mathrm{W} / \mathrm{O} / \mathrm{W}$ double emulsion particles which show very high monodispersity (Figure 18). 


\section{This content is unavailable.}

\section{Please consult the print}

version for access.

Figure 17: Schematic of a microfluidic device to produce double emulsion particles, reproduced from ${ }^{45}$

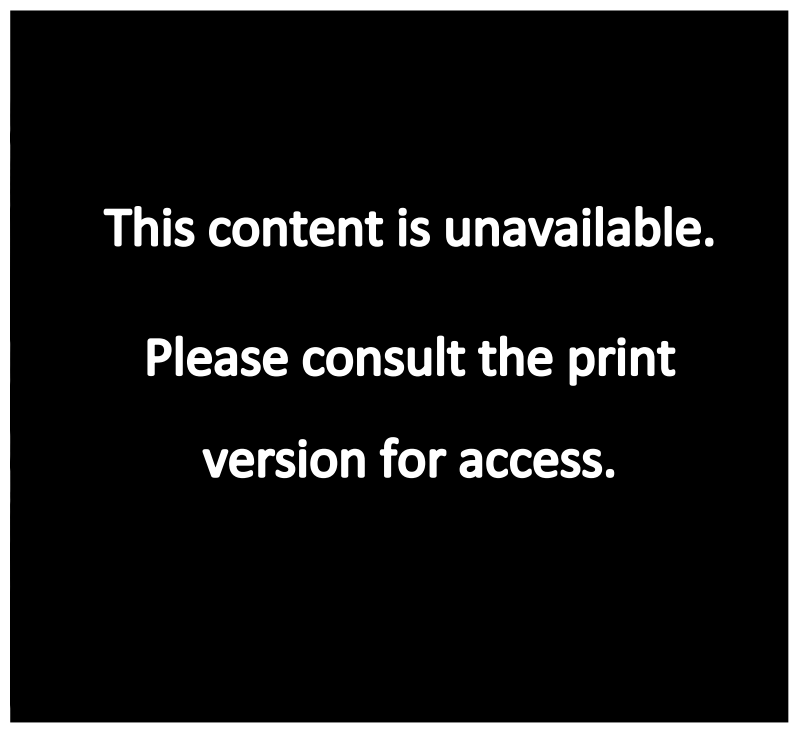

Figure 18: SEM micrograph of hollow cellulosic particles, reproduced from ${ }^{45}$

Monodisperse multiple emulsions can be formed using microfluidic systems and applied to particle formation for drug delivery. ${ }^{46}$ Ethyl cellulose was demonstrated to successfully encapsulate the active compounds. This application of microfluidics provides a highly controllable method with scale-up potential.

\subsubsection{Solvent diffusion}

Solvent diffusion is a process that can be utilised for the application of producing microparticles. The slow diffusion of solvent allows the nanoprecipitation of the polymer to occur as a monodisperse distribution of particles. The primary principle of particles generated from solvent diffusion. There are many different approaches for using solvent diffusion in the production of microparticles, including the 'Ouzo effect' ${ }^{47}$, dialysis ${ }^{48}$, solvent evaporation ${ }^{49}$ and solvent displacement ${ }^{49}$. All of these approaches are simply variations of the principle of solvent diffusion which produce solid particles 
with a suitable polymer dissolved into the organic phase. Two solvent diffusion processes will be discussed in the following section; 'Ouzo effect' and dialysis.

\subsubsection{1 'Ouzo effect'}

The use of solvent diffusion to produce hollow particles based on the 'Ouzo effect' is a very simple yet effective process. The 'Ouzo effect' gets its name from the Greek drink Ouzo that upon the addition of water, will spontaneously form particles from the anise oil dissolved in the Ouzo. This happens due to the supersaturation of anise oil in Ouzo that will nucleate as nanoparticles as an aqueous solution is poured into it. Particles generated from the 'Ouzo effect' are normally very monodisperse and sizes between $50-500 \mathrm{~nm}$ can be observed. The effect is described as the nanoprecipitation of a polymer by what can also be referred to as an anti-solvent precipitation. ${ }^{50}$ However, this differs from an antisolvent precipitation in which the addition of the solvent generates a stable emulsion; in the case of the 'Ouzo effect' it forms an unstable emulsion. The important advantage of the Ouzo effect is that it allows one to create relatively stable droplet dispersions, without the use of surfactants, stabilisers or any form of mechanical agitation. This contrasts with many of the other particle forming processes that utilise these components, which makes the 'Ouzo effect' a relatively green process in comparison.

A recent review by Elise Lepeltier ${ }^{51}$ and co-workers, discusses the use of the 'Ouzo effect' for the generation of nanocarriers for drugs. Here the biodegradable carrier is a desirable material as it can reduce toxic effects and allows for a greener process to be used. Figure 19 shows the process used to generate hollow nanoparticles using the simple 'Ouzo effect'.

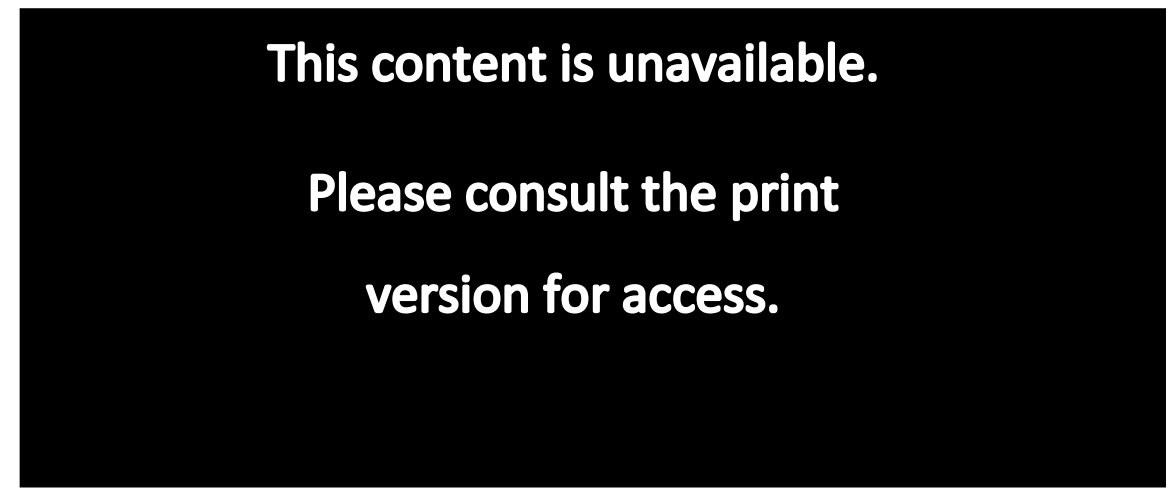

Figure 19: Nanoparticles made by the 'Ouzo effect', reproduced from ${ }^{51}$

The yellow region, shown in the middle diagram above, is what is known as the 'Ouzo region'. In this region, the polymer will come out of solution and form particles. In this domain, there is a large amount of water and polymer in the solution and only a small amount of solvent. As the solvent concentration in the solution decreases due to dilution, the polymer will precipitate out and a surfactant is used to stabilise the resulting emulsion. The primary factors controlling the process are the solvents of choice, temperature, $\mathrm{pH}$ and addition/diffusion rates. 


\subsubsection{Dialysis}

The dialysis method for producing nanoparticles uses the principle of solvent diffusion through a porous membrane. This then forces a polymer that is dissolved into the organic phase to nanoprecipitate, given that the organic phase is miscible with water.

Recent work ${ }^{49}$ by Thomas Heinze and co-workers investigated the use of three different solvent diffusion methods for the production of cellulosic nanoparticles. These methods included controlled precipitation, dialysis and emulsification evaporation. The dialysis method is of interest as it uses the principle of the 'Ouzo effect' through a porous cellulosic membrane to control the addition of solvent into the system. Figure $\mathbf{2 0}$ shows this process diagrammatically.

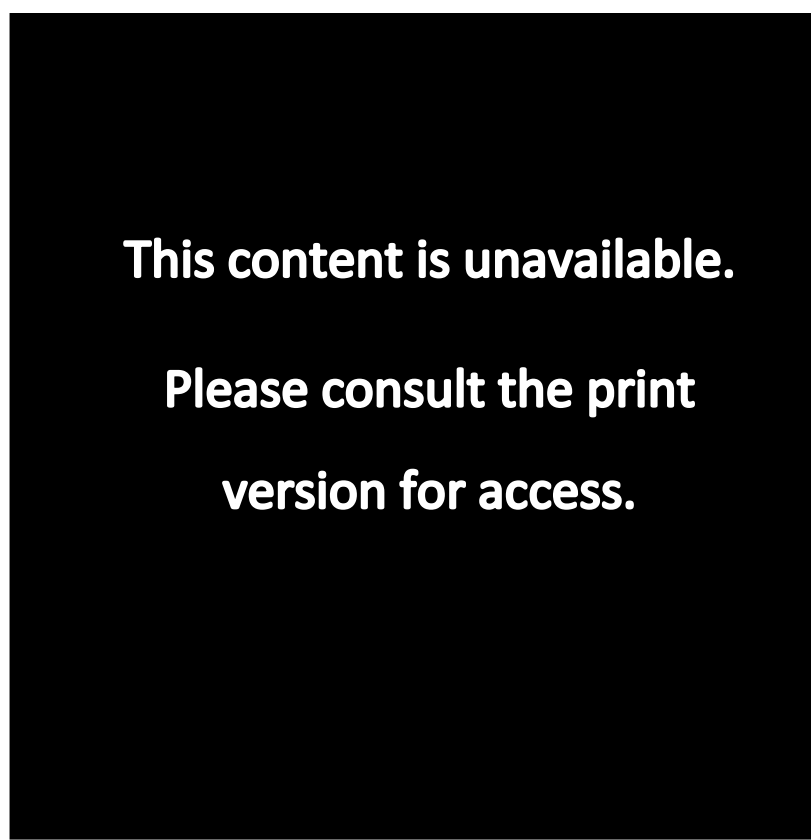

Figure 20: Dialysis method approach to cellulosic nanoparticles, reproduced from ${ }^{44}$

This process works by the diffusion of water through the porous membrane, where the cellulosic polymer and active compound are dissolved in an organic solvent. As the water diffuses in, and the solvent out of the membrane, the cellulosic polymer precipitates to form nanoparticles. The process was found to generate a moderately monodisperse mixture of particle sizes on the nanoscale. This process has the advantage of being very simple and green, and thus would be highly desirable to scale up. However, many factors would need to be considered to scale up this process, such as polymer concentration, solvent and diffusion time.

\subsubsection{Phase inversion}

Phase inversion is an emulsification technique that has low energy requirements in relation to traditional high energy emulsification methods, such as double emulsions. Here the dispersed phase is the internal phase and the continuous phase is the external phase (refer to figure 8). The process 
occurs with the slow addition of the dispersed phase to the continuous phase under sheer. During this addition the internal phase inverts to be the external phase; this process is mediated by surfactants, temperature and solvent compatibility. Figure $\mathbf{2 1}$ shows the process diagrammatically over time with addition of aqueous phase in this case.

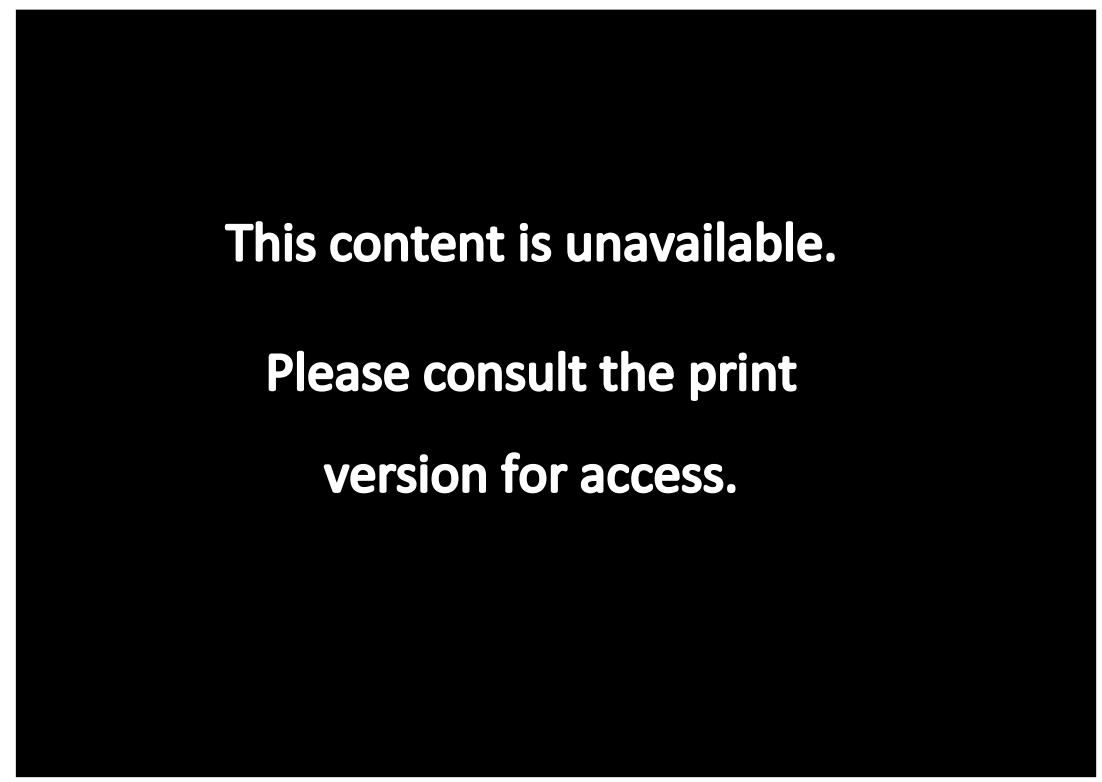

Figure 21: Phase inversion process, reproduced from ${ }^{52}$

In the above example, the phase being added is the water phase and during its initial addition the emulsion is a W/O, however after the first half of the water is added the emulsion inverts to an $\mathrm{O} / \mathrm{W}$ emulsion. The intermediate conditions of the emulsion go through a bicontinuous or lamellar phase where the phase interface goes through zero curvature before the phase inverts. This process can be finely tuned by careful selection of surfactants and phase selection, and the addition of salts to raise the ionic strength. An advantage of phase inversion over conventional emulsion techniques is that it can result in monodisperse and high internal phase volume emulsions, both of which are important characteristics for drug delivery systems.

An example of hollow particles produced by phase inversion emulsification is shown by work from 2010 by a group from Fudan University, Shanghai..$^{53}$ In this study they developed hollow particles using chlorinated polypropylene polymers grafted with methyl methacrylate, butyl acrylate and acrylic acid. The phase inversion emulsification proceeded by the slow addition of water to the polymer solution, which was made up of butanone, trimethylamine and the grafted polymer. Over time with the addition of water the emulsion inverts and forms solid hollow particles as the butanone diffuses into the water phase to precipitate the polymer. This is shown diagrammatically in Figure 22. 


\section{This content is unavailable.}

\section{Please consult the print version for access.}

Figure 22: Phase inversion process showing the diffusion of butanone, reproduced from ${ }^{53}$

The diffusion of the butanone is the key step to the formation of the solid hollow particles, and this is made possible due to the semi-miscibility of butanone with water. Thus, for the application of forming hollow particles using phase inversion emulsification, the ability for the non-solvent to diffuse into the water phase is a critical component.

\subsubsection{Mechanisms}

The phase inversion process may be explained by two different mechanisms, each of which lead to very different outcomes. These two mechanisms are known as catastrophic and transitional phase inversion. ${ }^{54}$ Catastrophic phase inversion occurs when the viscosity of the solution changes as a result of an increase in the volume fraction of the dispersed phase, whereas transitional inversion occurs when the surfactant distribution over the phases changes. The diagram shown in Figure $\mathbf{2 3}$ shows the different outcomes of these two mechanisms with respect to particle size distributions. 


\section{This content is unavailable.}

\section{Please consult the print}

version for access.

Figure 23: Particle size distributions of transitional and catastrophic phase inversion, reproduced from ${ }^{55}$

Catastrophic phase inversion leads to more polydisperse droplets and is a less controlled process. On the other hand, transitional phase inversion is more controllable and can lead to highly monodisperse droplets. Therefore, to obtain particles that are monodisperse using phase inversion, the transitional mechanism is favoured. Investigation into the nature of phase inversion mechanisms was studied in a recent work from $2006 .{ }^{56}$ In this work they used a model cyclohexane-in-water emulsion system with two polyoxyethylene nonylphenyl ether surfactants, which were soluble in water or organic solution. Using this model emulsion, they found the drop size was highly dependent on which of the two mechanisms predominated in the system. For catastrophic inversion they found the drop size peak was higher and the distribution broader than that of transition inversion. Their results are shown in the graph Figure 24 below.

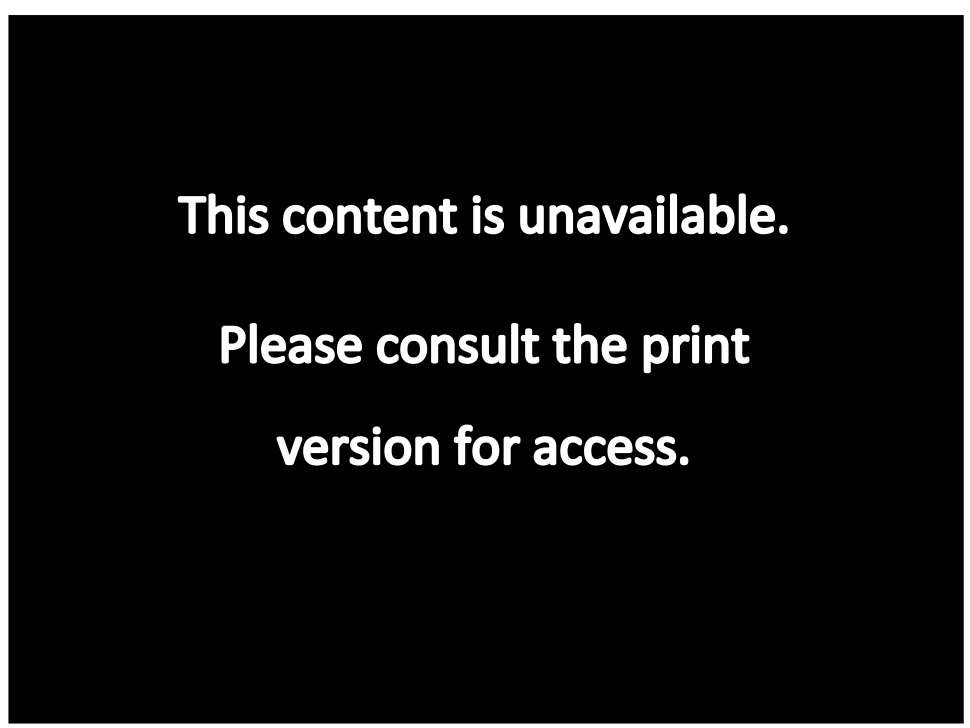

Figure 24: Particle size distributions of different paths to emulsification, reproduced from ${ }^{56}$

The different distributions represent the different paths that were taken, in terms of the order of mixing and the phase containing the surfactant. Paths $P$ and $A O$ represent phase inversion, with $P$ 
going through transitional and $A O$ going through catastrophic. Paths $D$ and $A W$ represent direct emulsion where the oil phase was added to the water continuous phase, with the location of the surfactant the only difference. The distributions attained from the two phase inversion paths show the distinct difference in particle size distributions between the two mechanisms. The study was able to conclude that transitional phase inversion emulsification is a far better option for making fine emulsions in comparison to the other routes studied in this work.

Two main conditions can be manipulated to direct which inversion path dominates. These are surfactant concentration and addition time of the discontinuous phase. For longer addition times, up to 60 seconds, transitional phase inversion could be favoured over catastrophic. ${ }^{57}$ Along with the same trend being true for higher surfactant concentrations, and sometimes to favour transitional inversion, a combination of the two would be required. It was concluded that the reasoning behind this was that with longer addition times a thermodynamic semi-equilibrium could be achieved, if the optimal compositions of phases were achieved at the same time. This would lead to the more thermodynamically favoured transitional inversion and the shorter addition times would lead to catastrophic inversion that is more kinetically favoured. The study also showed that these two mechanisms of phase inversion could occur at the same time if the conditions allowed it. This would lead to a bimodal distribution as the transitional phase inversion leads to a population of smaller particles with a sharp peak, and catastrophic inversion leads to a much broader peak with larger particle sizes. Therefore, each mechanism is not mutually exclusive to the other and depending on the conditions of the formulation, both mechanisms can occur at the same time.

\subsubsection{Salting out}

Salting out is a process that uses high ionic strength solutions to precipitate out products or dissolve a product that can then be precipitated by lowering the ionic strength of the solution. This process is similar to that of anti-solvent recrystallization, where two different solvents are used to control the precipitation of a product in order for it to crystallise successfully. This process is often used for the crystallisation of industrially produced chemical products and protein isolation. ${ }^{58}$ It can be applied to the phase inversion emulsification process to allow the different solubility of the polymers and active compound/s to be incorporated into a solid particle upon precipitation of the polymer. In Figure 25 a generic process for forming solid particles using salting out is demonstrated. 


\title{
This content is unavailable.
}

\section{Please consult the print}

\author{
version for access.
}

Figure 25: Salting out procedure using phase inversion emulsification, reproduced from ${ }^{59}$

Once an emulsion is formed between the organic and water phases, the addition of excess water causes the acetone to diffuse out of the organic phase, thus the polymer in the organic phase will precipitate forming hollow particles. In the case above, the use of acetone is critical as acetone is miscible in water, thus when a saturated salt solution is emulsified with an acetone phase they will form an emulsion due to the change in solubility of a salt solution. However, once the ionic strength is reduced by the addition of excess water the acetone can diffuse into the water.

Encapsulation of a sunscreen agent demonstrated that the process was viable for a slow release application. ${ }^{60}$ Ethyl cellulose was used as the polymer and the active compound was 2-ethylhexyl-pmethoxycinnamate, known to be an active ingredient in sunscreen. Good particle morphologies and relatively low dispersity were achieved (Figure 26).

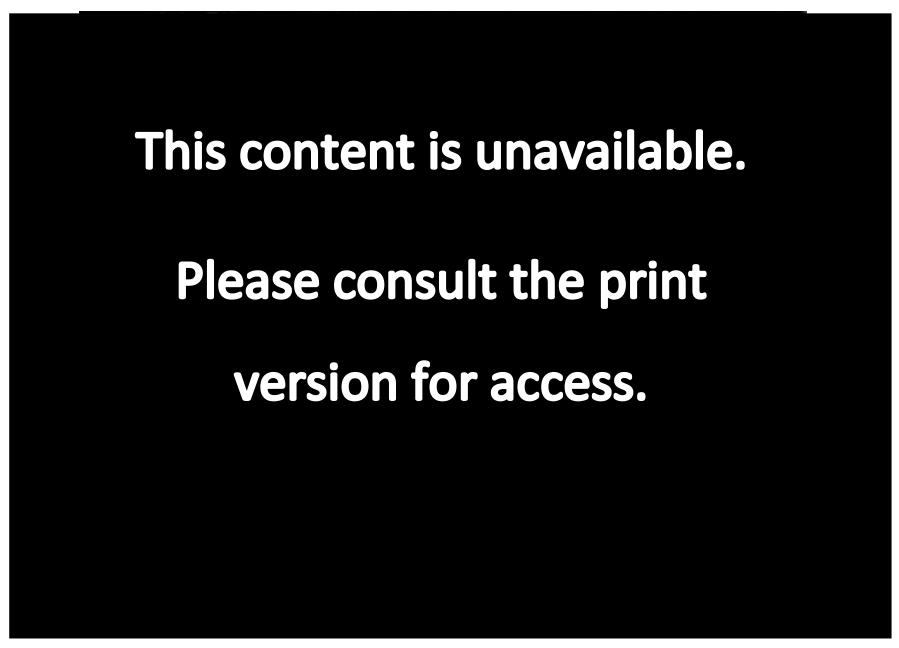

Figure 26: SEM micrograph of ethyl cellulose particles by 'salting out', reproduced from ${ }^{60}$ 
This compound was encapsulated using both 'salting out' and phase inversion techniques. The aqueous phase used polyvinyl alcohol as the surfactant and calcium chloride as the salt in the aqueous phase and acetone was used to dissolve the polymer for the organic phase. Acetone and water are miscible in standard conditions, thus would not be suitable solvents for forming emulsions. However, the use of a saturated salt solution changes the solubility profile of the aqueous phase thus allowing these two solvents to be immiscible and form an emulsion. The method used in this study also utilised the high ionic strength of the aqueous phase to allow controlled precipitation of the polymer, by the addition of excess water after the emulsion was formed by phase inversion. The polymers tested in the study were ethyl cellulose (E-cell) and poly-D,L-lactide-co-glycolide (PLGA). They found that the PLGA formed particles had the best encapsulation efficiency over E-cell along with more uniform particle size distributions. They also noted that the inclusion of the active sunscreen agent did not have any significant impact on particle morphologies and size distributions.

\subsection{Wood treatments and biocide testing}

Biocides used for protection of wood based products are a vital component to the reduction of the natural biodegradation of wood in commercial applications. ${ }^{61}$ However, these biocides must first be tested, not only to quantify their efficacy but also toxicity to non-target organisms. Another aspect is the leaching of the active components from the wood and their respective off target toxicity.

\subsubsection{Chromated copper arsenate (CCA)}

One of the most commonly used wood preserving agents used is CCA. This formulation is a combination of three metals as oxides or salts where each of the metals has a specific role in the mode of action. The chromium is a chemical fixing agent - it binds the lignin and cellulose in the wood, the copper acts as the primary biocide against fungi and/or bacteria and the arsenic is insecticidal and acts against termites. CCA was first introduced in the $1933^{62}$ and has been implemented as a wood preserving formulation since. It is an effective formulation, however the heavy metals used can be very hazardous to off-target organisms. Many studies ${ }^{63-65}$ have been carried out on the toxicity of CCA, most of which have generated conflicting data, but it is generally recognised that CCA is harmful to marine life. This is mediated by the leaching of CCA from the wood. A review on this showed the leaching of CCA to be higher than previous studies had shown and proposed the standardisation of leach testing protocols. ${ }^{66}$

\subsubsection{Borate preservatives}

Borate preservatives are another very common biocide used for the protection of wood. Borates are oxyanions of boron and can exist in various different forms, such as boric acid and polymeric borates. 
The formulations have become much more complex over time, with many of them including copper, chromium, quaternary ammonium or organic ligands. Borate wood preservatives have the advantage of being relatively benign to humans and other terrestrial organisms, however it has the disadvantage of not being chemically fixed to the wood. Long term studies on the potency of borate wood preservatives, have shown them to be very effective against a variety of wood degrading targets. ${ }^{67}$ Leaching is the primary downside to borate preservatives. The reduction of borate leaching in commercial applications ${ }^{68}$ showed that when borates are used in exterior applications (where water can wash over it) will cause the borate formulation to leach from the wood at a very high rate compared to other applications.

\subsubsection{Propiconazole-tebuconazole-imidacloprid (PTI)}

PTI wood preservatives are formulations containing three organic fungicides and insecticides as the active ingredients. This formulation was developed as a response to the potential for metallic based preservatives to have harmful off target effects. All three of these biocides are commonly used in food crop applications, thus there was no need to approve these for use in wood preserving. PTI has the primary advantage of not carrying any metallic components in the formulation, whereas the other two discussed here use metallic components as primary additives. Introducing new compounds for use as wood preserving formulations is difficult due to current regulations, ${ }^{69}$ therefore much of this area uses compounds or formulations that are well studied.

\subsubsection{Assessment of wood preservatives in Australasia}

The assessment of any potential wood preservative in Australasia must conform to the guidelines as presented by the Australasian Wood Preservation Committee (AWPC). These guidelines were first introduced in 1997, and included procedures for all hazard classes ${ }^{70}$ (Table 2), with the latest incarnation of these guidelines being updated in 2015. The hazard classes corresponding to termite infections are; $\mathrm{H} 1, \mathrm{H} 2, \mathrm{H} 3, \mathrm{H} 4$ and $\mathrm{H} 5$, whereas the hazard classes corresponding to wood fungi are; $\mathrm{H} 1.2, \mathrm{H} 3, \mathrm{H} 4$ and $\mathrm{H} 5$. The standard testing procedure for these hazard classes is covered in the AWPC protocols 2015. 
Table 2: Hazard class selection guide, adapted from AWPC protocols ${ }^{70}$

\begin{tabular}{|c|c|c|c|c|}
\hline Hazard class & Exposure & $\begin{array}{l}\text { Specific service } \\
\text { conditions }\end{array}$ & Biological hazard & Typical uses \\
\hline H1 & $\begin{array}{l}\text { Outside, } \\
\text { above ground }\end{array}$ & $\begin{array}{l}\text { Completely } \\
\text { protected from the } \\
\text { weather and well } \\
\text { ventilated and } \\
\text { protected from } \\
\text { termites }\end{array}$ & $\begin{array}{l}\text { Insects other } \\
\text { than termites } \\
\text { (lyctines in } \\
\text { Australia, } \\
\text { and lyctines and } \\
\text { anobiids in } \\
\text { New Zealand) }\end{array}$ & $\begin{array}{l}\text { Framing, } \\
\text { flooring, } \\
\text { furniture, } \\
\text { interior joinery }\end{array}$ \\
\hline H1.2 New Zealand only & $\begin{array}{l}\text { Inside, } \\
\text { above ground }\end{array}$ & $\begin{array}{l}\text { Inadequately } \\
\text { protected from the } \\
\text { weather, and risk } \\
\text { of moisture } \\
\text { entrapment/nondrying }\end{array}$ & Decay & House farming \\
\hline $\mathrm{H} 2$ and $\mathrm{H} 2 \mathrm{~F}_{\text {Australia only }}$ & $\begin{array}{l}\text { Inside, } \\
\text { above ground }\end{array}$ & $\begin{array}{l}\text { Protected from } \\
\text { wetting. Nil } \\
\text { leaching }\end{array}$ & $\begin{array}{l}\text { Borers } \\
\text { and termites }\end{array}$ & $\begin{array}{l}\text { Framing, } \\
\text { flooring, etc. } \\
\text { used } \\
\text { in dry situations }\end{array}$ \\
\hline $\begin{array}{l}\text { H3 Australia } \\
\text { H3.1 and H3.2 New Zealand }\end{array}$ & $\begin{array}{l}\text { Outside, } \\
\text { above ground }\end{array}$ & $\begin{array}{l}\text { Subject to } \\
\text { periodic wetting } \\
\text { and leaching }\end{array}$ & $\begin{array}{l}\text { Moderate } \\
\text { decay, borers } \\
\text { and termites }\end{array}$ & $\begin{array}{l}\text { Weatherboard, } \\
\text { fascia, window } \\
\text { joinery and } \\
\text { decking }\end{array}$ \\
\hline H4 & $\begin{array}{l}\text { Outside, } \\
\text { in ground }\end{array}$ & $\begin{array}{l}\text { Subject to } \\
\text { severe wetting } \\
\text { and leaching }\end{array}$ & $\begin{array}{l}\text { Severe } \\
\text { decay, borers } \\
\text { and } \\
\text { termites }\end{array}$ & $\begin{array}{l}\text { Fence posts, } \\
\text { greenhouses, } \\
\text { pergolas and } \\
\text { landscaping } \\
\text { timbers }\end{array}$ \\
\hline H5 & $\begin{array}{l}\text { Outside, } \\
\text { in ground } \\
\text { contact, with } \\
\text { or in fresh } \\
\text { water }\end{array}$ & $\begin{array}{l}\text { Subject to } \\
\text { extreme wetting } \\
\text { and leaching } \\
\text { and/or where the } \\
\text { critical use requires } \\
\text { a higher degree } \\
\text { of protection }\end{array}$ & $\begin{array}{l}\text { Very severe } \\
\text { decay, borers } \\
\text { and termites }\end{array}$ & $\begin{array}{l}\text { Retaining walls, } \\
\text { piling, house } \\
\text { stumps, building } \\
\text { poles, cooling } \\
\text { tower fill }\end{array}$ \\
\hline H6 & $\begin{array}{l}\text { Marine waters } \\
\text { exposure }\end{array}$ & $\begin{array}{l}\text { Subject to } \\
\text { prolonged } \\
\text { immersion in } \\
\text { sea water }\end{array}$ & $\begin{array}{l}\text { Marine wood } \\
\text { borers } \\
\text { and decay }\end{array}$ & $\begin{array}{l}\text { Marine piles, } \\
\text { jetty cross- } \\
\text { bracing, } \\
\text { landing steps, etc }\end{array}$ \\
\hline
\end{tabular}

There are various different test procedures used, and the scale of these will depend on the level of development of the wood preservative being tested. Larger scale test procedures will use treated wood blocks that are constructed to form relevant building structures. The testing procedures relevant to this project are the small-scale tests; the primary test procedure for a new wood preservative will use the 'rot jar test' (Figure 27). 


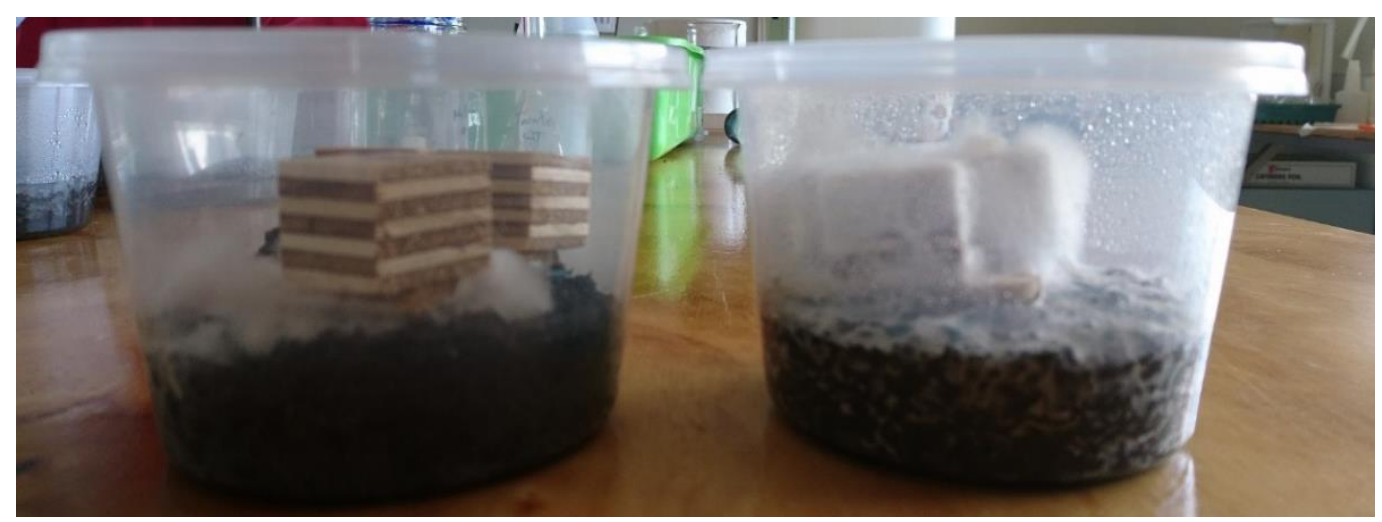

Figure 27: 'rot jar test' of plywood blocks against wood fungi

This will involve a selection of wood species, some inoculated with the preservative and others without, to act as the control. Each jar will have either 10 grams of termites inside or 10 grams of the select wood fungi species. Under ideal growing conditions, the termites or fungi will degrade the wood and the effectiveness of the preservative to impede this degradation is measured by the weight difference in the sample over a set period of time. This weight difference will indicate the quantity of wood mass degraded over the time period, thus showing how well the wood preservative has worked.

\section{Results and discussion}

\subsection{Interfacial polymerisation}

The initial body of work carried out in this project was investigating particle formation using interfacial polymerisation. This method uses two monomers present in both phases of an emulsion, which under the correct conditions will undergo a polycondensation reaction to form a polymer. This reaction will occur at the phase interface of the emulsion that has been formed, thus forming a solid polymeric particle that is hollow and encapsulates the organic phase. The basis for this work was the industrial process of forming polyurea particles using an interfacial polymerisation method (Figure 28), where the inclusion of cellulose acetates would be investigated. The inclusion of cellulose acetates was hypothesised to make these particles a target for termites and/or wood fungi when applied into a wood glue system that would aid in preventing proliferation of these pests. 


\section{This content is unavailable.}

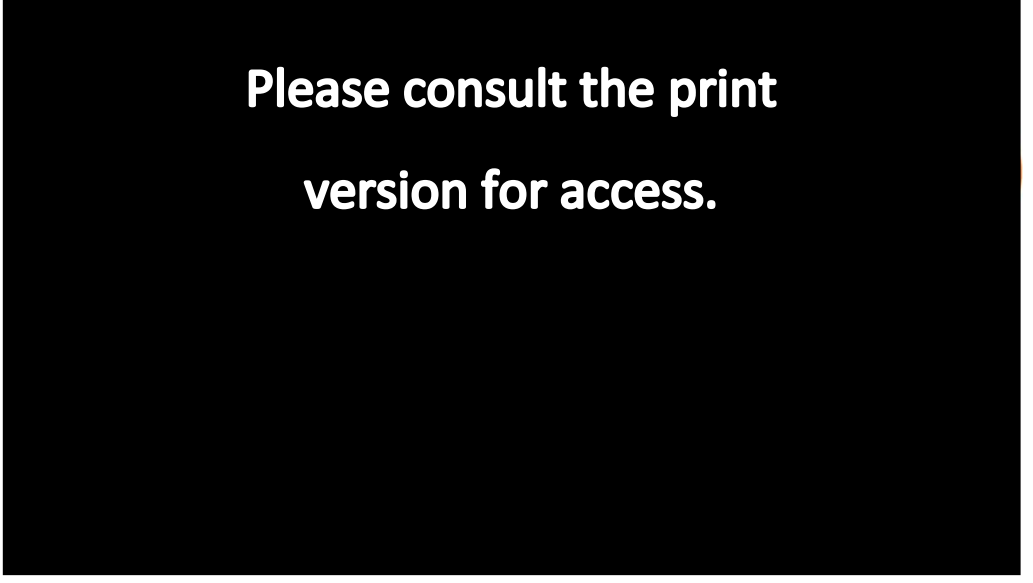

Figure 28: Interfacial polymerisation particle forming process, reproduced from ${ }^{71}$

In the above diagram the oil phase will contain the active compound along with the diisocyante. This is mixed under shear into the water phase with the appropriate surfactant present, forming the oil in water emulsion. Once the emulsion has been formed, the diamine is added and the solution is heated. This promotes the reaction of the two monomers which occurs at the oil water interface, forming a solid polymeric shell around the active compound. The reaction that takes place is a polycondensation reaction, where an amine reacts with an isocyanate functional group at an elevated temperature of approximately $60^{\circ} \mathrm{C}$. The reaction will form a carbamate between the two $\mathrm{R}$ groups and because a diisocyanate and a diamine are used, the reaction will form a long chain polyurea polymer.

One method to include cellulose into the polymer matrix of these particles is to use a water soluble cellulosic. It was desirable to have an esterified cellulose as it had been reported these demonstrated some decomposition under fungal action and did not affect the solution viscosity as in the case of water soluble hydroxyl cellulose ethers or cellulosic carboxy species. As water soluble cellulose esters are not readily available, the first step was to generate a cellulose acetate with a degree of substitution (DS) such that it was water soluble (DS=0.5-0.9). To achieve water soluble cellulose acetate, the DS must be below that of any commercially available compound. Once this modified cellulose acetate was made, the particles were formulated without any additives to observe their morphology and particle sizes. Finally, the inclusion of additives to the formulation such as chitosan, methyl cellulose and the modified cellulose acetate were investigated. The morphologies and particle sizes of all 
particle forming experiments were analysed by SEM and glass transition temperatures of the novel polymers were analysed by DSC.

\subsubsection{Preparation of water soluble cellulose acetate (CA)}

The first step was to synthesis a water soluble cellulose acetate, so it could be incorporated into the aqueous phase of the particle forming experiments. Cellulose acetate can have different solubility profiles depending on its degree of substitution of acetates to hydroxyl groups. ${ }^{72}$ The different solubilities achieved are shown in Figure 29. The degree of substitution refers to the number of acetates per monomer as an average over the whole polymer ( 3 is the maximum for cellulose acetates).

\begin{tabular}{|c|c|c|c|c|}
\hline Product & $\begin{array}{l}\text { Worldwide } \\
\text { production (t/a) }\end{array}$ & Functional group & $\begin{array}{l}\text { Degree of } \\
\text { functionalisation }\end{array}$ & $\begin{array}{l}\text { Examples of } \\
\text { solubility }\end{array}$ \\
\hline \multirow[t]{4}{*}{ Cellulose acetate } & \multirow[t]{4}{*}{900,000} & \multirow[t]{4}{*}{$-\mathrm{C}(\mathrm{O}) \mathrm{CH}_{3}$} & $0.6-0.9$ & Water \\
\hline & & & $1.2-1.8$ & 2-Methoxy ethanol \\
\hline & & & $2.2-2.7$ & Acetone \\
\hline & & & $2.8-3.0$ & Chloroform \\
\hline \multirow[t]{2}{*}{ Cellulose nitrate } & \multirow[t]{2}{*}{200,000} & \multirow[t]{2}{*}{$-\mathrm{NO}_{2}$} & $1.8-2.0$ & Ethanol \\
\hline & & & $2.0-2.3$ & Methanol \\
\hline Cellulose xanthate & $3,200,000$ & $-\mathrm{C}(\mathrm{S}) \mathrm{SNa}$ & $0.5-0.6$ & Aqueous $\mathrm{NaOH}$ \\
\hline $\begin{array}{l}\text { Carboxymethyl } \\
\text { cellulose }\end{array}$ & 300,000 & $-\mathrm{CH}_{2} \mathrm{COONa}$ & $0.5-2.9$ & Water \\
\hline \multirow[t]{3}{*}{ Methyl cellulose } & \multirow[t]{3}{*}{150,000} & \multirow[t]{3}{*}{$-\mathrm{CH}_{3}$} & $0.4-0.6$ & $4 \%$ Aqueous $\mathrm{NaOH}$ \\
\hline & & & $1.3-2.6$ & Cold water \\
\hline & & & $2.5-3.0$ & Organic solvents \\
\hline \multirow[t]{3}{*}{ Ethyl cellulose } & \multirow[t]{3}{*}{4,000} & \multirow[t]{3}{*}{$-\mathrm{CH}_{2} \mathrm{CH}_{3}$} & $0.5-0.7$ & $4 \%$ Aqueous $\mathrm{NaOH}$ \\
\hline & & & $0.8-1.7$ & \multirow{2}{*}{$\begin{array}{l}\text { Cold water } \\
\text { Organic solvents }\end{array}$} \\
\hline & & & $2.3-2.6$ & \\
\hline Hydroxyethyl & 50,000 & $-\mathrm{CH}_{2} \mathrm{CH}_{2} \mathrm{OH}$ & $0.1-0.5$ & $4 \%$ Aqueous $\mathrm{NaOH}$ \\
\hline cellulose & & & $0.6-1.5$ & Water \\
\hline
\end{tabular}

Figure 29: Table of solubilities of cellulose acetate with respect to DS, adapted from ${ }^{72}$

The table shows that a degree of substitution of 0.6-0.9 for cellulose acetate should make the polymer soluble in water, so this degree of substitution was the target of this synthesis. The building blocks used for this synthesis were the two commercially available cellulose acetates CA 320-S and CA 3983 , with degrees of substitution of 1.8 and 2.5 respectively. Given these are higher than the target, a reaction that would selectively deacetylate the polymer was investigated. 


\subsubsection{Methanol iodine deacetylation}

The first reaction attempted for the deacetylation of cellulose acetate used iodine to deacetylate the polyester in methanol. ${ }^{73} \mathrm{It}$ is proposed that primary acetates are most susceptible to elimination due to steric considerations (Figure 30).

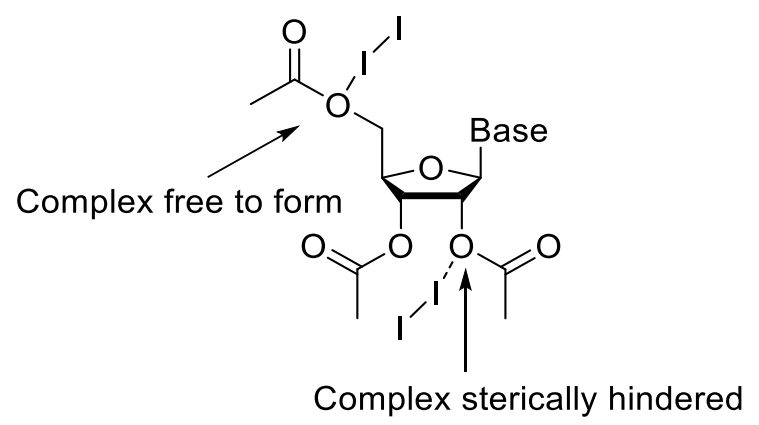

Figure 30: Proposed interactions for deacetylation, lodine methanol process, adapted from ${ }^{73}$

The proposed interactions shown above demonstrates the iodine-methanol process is selective for the elimination of primary acetates. The secondary acetates are sterically hindered and cannot form the iodine complex needed for the reaction to proceed. The primary acetate carbonyl is readily accessible to iodine and has the lowest steric hindrance to nucleophilic attack by methanol to hydrolyse the acetate.

Cellulose acetate was dissolved in DMSO, then solid iodine was dissolved into methanol and the solutions were combined. The reaction was carried out in a sealed reaction vessel for 12 hours at $80^{\circ} \mathrm{C}$. The reaction mixture was cooled to room temperature and quenched with sodium thiosulfate and a white precipitate was observed. This cloudy solution was then evaporated to dryness using methanol to co-evaporate the water. Once the solid was dried, proton nuclear magnetic resonance spectroscopy $\left({ }^{1} \mathrm{H}\right.$ NMR) revealed that the product was not appreciably modified from the starting material. No deacetylation had been affected, as evidenced by the product precipitating out in water, demonstrating it did not have the correct solubility properties. Further experiments were conducted at $120^{\circ} \mathrm{C}$ for 24 hours in the same sealed reaction vessel. Once these reactions were quenched, no precipitate was observed. The product was then isolated by co-evaporation with methanol and a proton NMR recorded (Figure 31). This revealed the reaction had caused some deacetylation to the cellulose, however as the peaks were no longer broad, the reaction had likely depolymerised the cellulose into discrete monomers along with deacetylating it. 


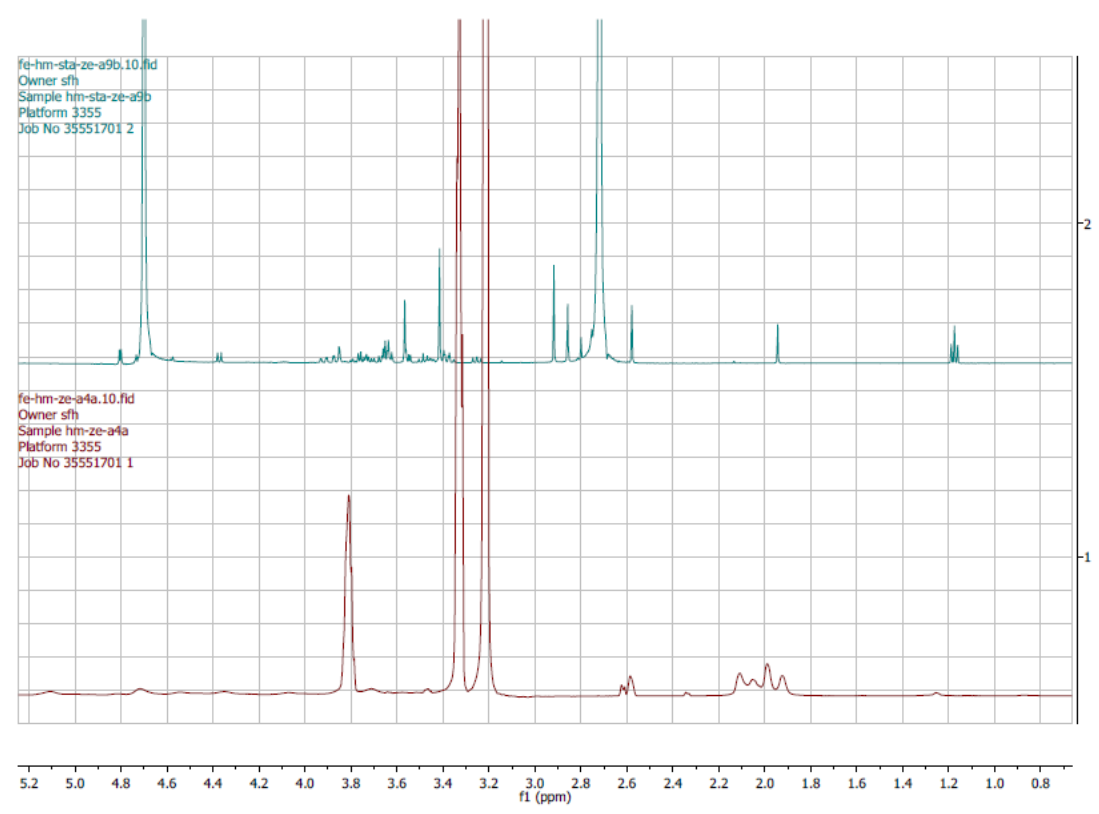

Figure 31: ${ }^{1} \mathrm{H}$ NMR $\left(\mathrm{D}_{2} \mathrm{O}\right)$ of modified cellulose acetate, depolymerised (top) and unreacted (bottom)

Polymeric species exhibit broad spectra due to both inter- and intra-molecular interactions that give rise to multiple environments for individual atoms on repeating units. In addition, on the polymer backbone different environments are experienced due to different substitution patterns for species that are not fully substituted ( $D S=3$ ). For oligomeric species end-group effects can also give rise to broadened spectra. The sharp and defined spectral features are either the result of a polymer with the same repeating monomer, or due to reduction to small identical oligomeric species. This method was not further investigated due to the observation that after 12 hours there was no reaction, and after 24 hours complete depolymerisation and degradation of the polymer had occurred; hence it was unlikely that a CA of defined DS could be reproducible prepared. In addition, the unintended depolymerisation was a concern.

\subsubsection{Acid hydrolysis}

An acid hydrolysis reaction was attempted. Dissolution of the cellulose polymer in a mixed solvent system of methanol and acetic acid was completed before a portion of $3 \mathrm{M}$ sulfuric acid was added to the solution and heated to reflux for 6 hours. ${ }^{74}$ The initial reactions showed no discernible change to the composition of the cellulose, so changes to the reaction were made after several attempts with the two different cellulose starting materials. First change that was made was increasing the time period was increased to 16 hours, with no change to the other conditions. This yielded a lower DS cellulose product, however only the lower DS starting material (CA 320-S) gave the target DS of 0.50.7. A proton NMR of the polymer is shown in Figure 32. 


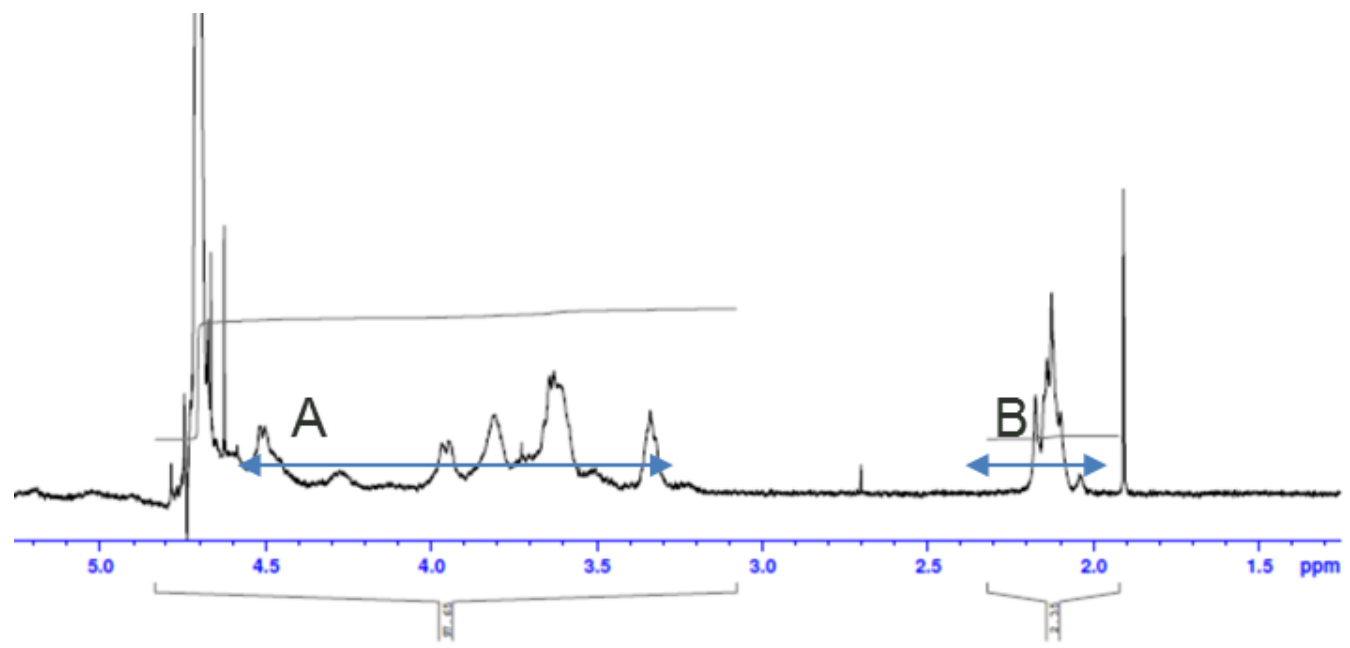

Figure 32: ${ }^{1} \mathrm{H}$ NMR $\left(\mathrm{D}_{2} \mathrm{O}\right)$ of water soluble cellulose acetate, intergrated areas for the calculation of DS shown The area marked $A$ is the protons of the cellulose unit, whereas the area marked $B$ is the protons that represent the methyl groups on the acetates. By calculating the integration of these two areas, finding the ratio of the two and dividing by the number of protons per area, one can identify the exact DS of the polymer, using the following formula; $(B / 3) /(A / 7)=D S$. This formula yielded a DS of 0.56 for the product attained in this spectrum. The spectrum of the water soluble cellulose acetate differs from the parent polymer (CA 320-S) as the peaks are much less prominent (Figure 33).

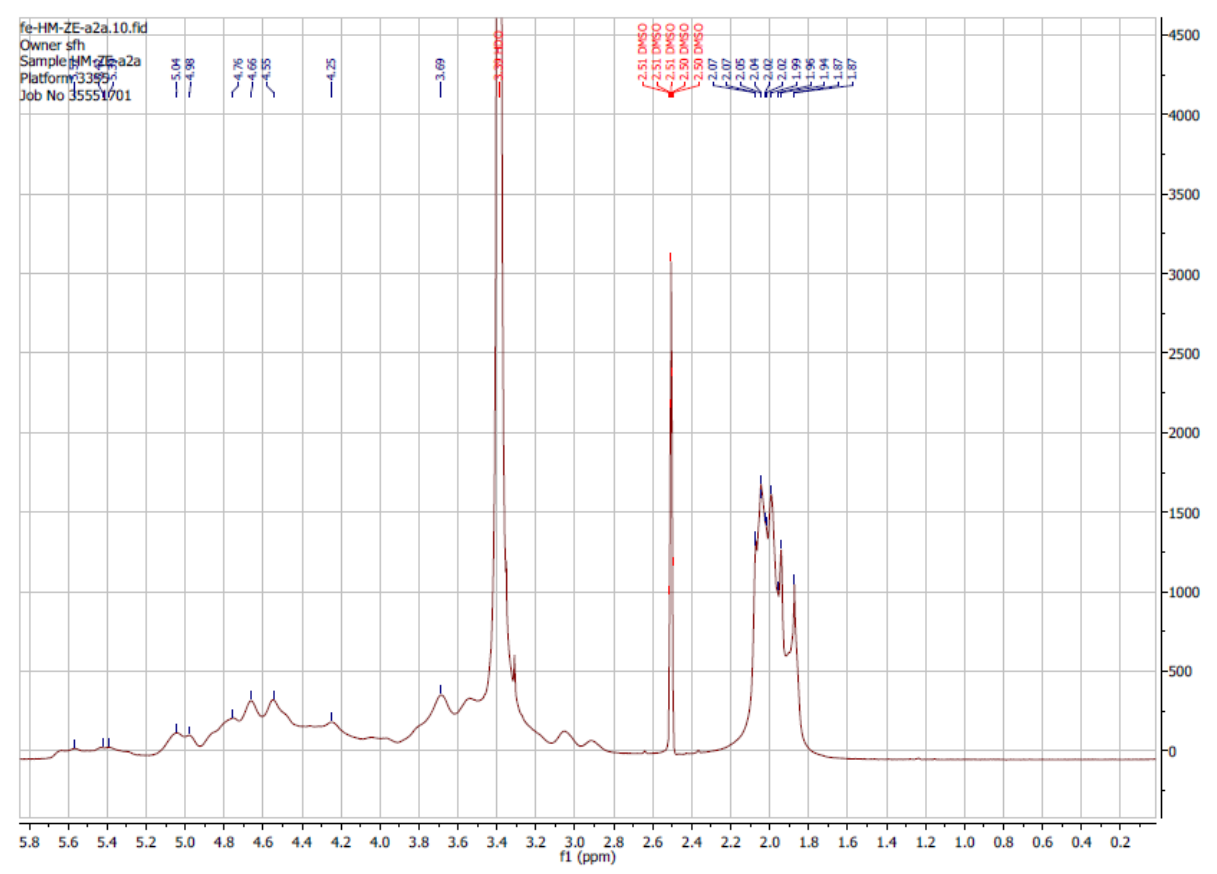

Figure 33: ${ }^{1} \mathrm{H}$ NMR (D6-DMSO) of cellulose acetate polymer CA 320-S

Although the spectra of the water-soluble cellulose was taken in $\mathrm{D}_{2} \mathrm{O}$ whereas the polymer CA 320-S was taken in $D^{6}$-DMSO which does change where the peaks are recorded, a comparison can be readily 
made for this compound with the large separation between the acetate resonances and the rest of the polymer spectral features. The primary difference is in the ratio of the acetate to anhydroglucose peaks, which is dependent on the number average of acetates on the cellulose monomer. Given the success of this reaction a larger scale reaction was set up to produce adequate material for testing in the particle formulations.

\subsubsection{Blank particles}

A method was generated to use the chemicals provided by our industry partners Lonza, with polymeric diioscyanate and diethyl triamine, used as monomers 1 and 2 respectively (in reference to Figure 11). A reaction scheme for the interfacial polymerisation reaction carried out in these experiments is shown in Figure 34.<smiles>CC(Cc1cc([N+](=O)[O-])cc(C(C)(C)C)c1)c1cccc([N+](=O)[O-])c1</smiles>

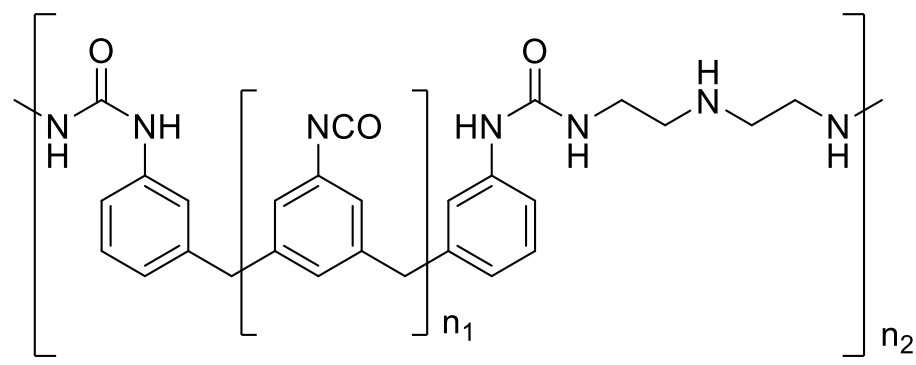

Figure 34: Reaction scheme for the interfacial polymerisation of PDI and diethyl triamine

The initial interfacial polymerisation experiments were completed without any active components (insecticides or cellulosics). This was to provide particles as a reference and to observe what morphology an 'unloaded' or 'blank' particle would assume. The method was done on a $10 \mathrm{~mL}$ scale to reduce solution wastage,$^{75}$ and these initial experiments used a reaction time of approximately 3 minutes. The process starts with the formation of an emulsion with $8 \mathrm{mLs}$ of aqueous phase (which contains $10 \mathrm{w} / \mathrm{w} \%$ polyvinyl acetate (PVA) and sodium lignosulfonates) and $2 \mathrm{mLs}$ of aromatic 200 (which contains xylene and naphthalene, with other aromatic solvents as a mixture) with $0.06 \mathrm{mLs}$ of polymeric diisocyanate (PMDI) dissolved in it. This emulsion is then heated to $60^{\circ} \mathrm{C}$ and sheer applied to the system. During this step, diethyl triamine (DETA) is added and the reaction proceeds over 3-5 minutes. The particles are then formed, and they are filtered over cellulose acetate filter paper under vacuum. The particles generated were analysed using SEM to assess particle morphology (Figure 35). 

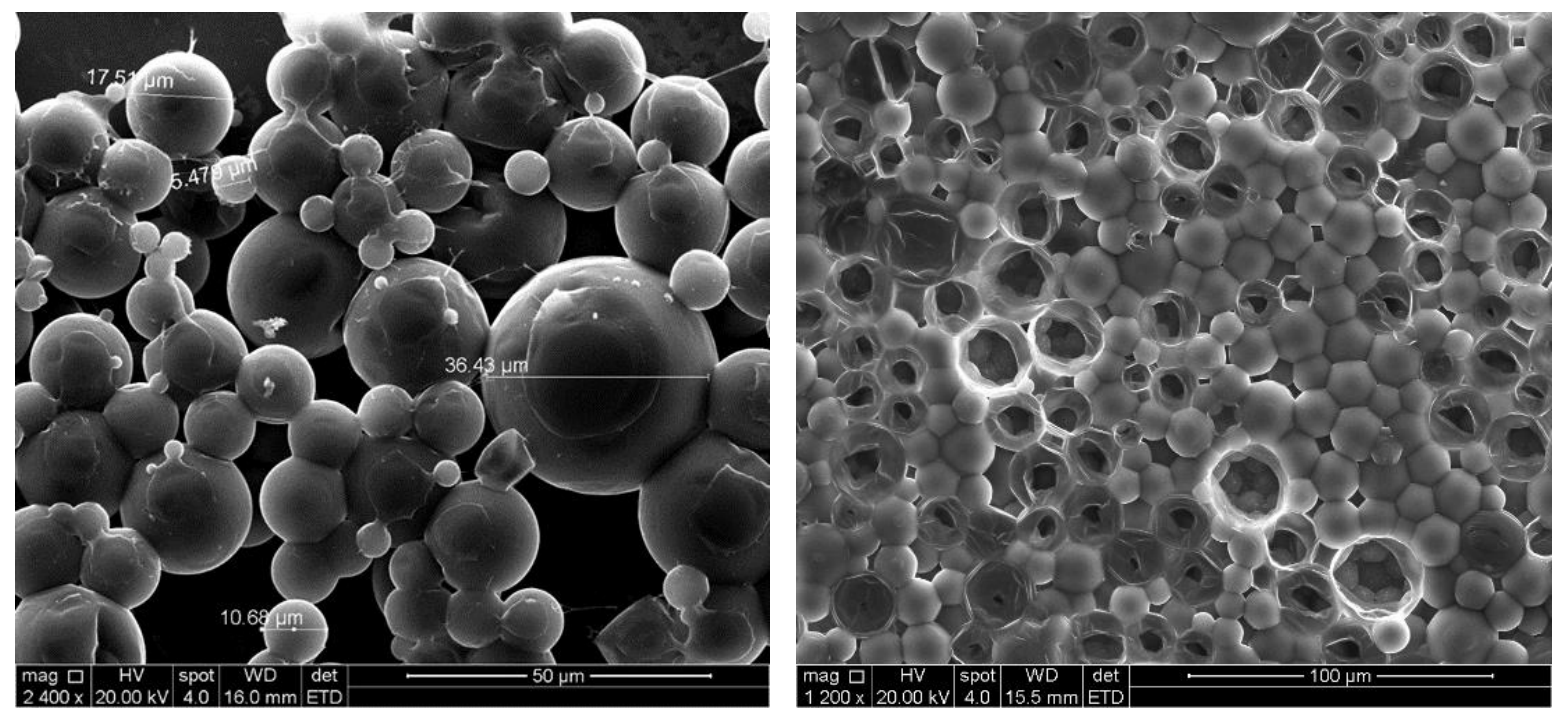

Figure 35: SEM images of blank particles produced using interfacial polymerisation

Many of the particles burst under the conditions of preparation and recording the SEM (see image on right). This is likely due to the low glass transition temperature $\left(T_{g}\right)$ of the polymer, which results in a particle that is more easily deformed at room temperature. When the particles are dried on the SEM stub, the solvent inside the particles boils and can burst the particles. The particle sizes found under the SEM ranged from $10 \mu \mathrm{m}$ to $40 \mu \mathrm{m}$. This distribution was larger than what was reported previously, ${ }^{75}$ where a particle size average of $1 \mu \mathrm{m}$ was observed, but they also state there is a large population of smaller particles that have an average size of approximately $0.1 \mu \mathrm{m}$. However, they do not state if a dynamic light scattering machine was used to give this value or if they simply measured the sizes from the SEM images. Thus, it is difficult to conclude why their experimental data differs from that generated in this work. One possible reason why the samples generated in this project had such a wide distribution of particle sizes is the time period for which the emulsion is under shear once the reaction vessel is heated. Increased shear would be expected to generate smaller particle sizes. Also, longer reaction time would be expected to lead to larger particles (through Ostwald ripening) and size distributions (if continued reaction was occurring).

\subsubsection{Incorporation of carbohydrate polymers}

Several repeat experiments to produce blank particles indicated that they could be manufactured in a reproducible fashion. Additives were incorporated into the particle formulation to observe the effects on the system. Methyl cellulose was used first as a model compound as it is water soluble and thus of similar solubility to the modified cellulosed acetates. Chitosan was then attempted to be used as an additive, and lastly the modified cellulose acetates were used. The samples were analysed by SEM to observe morphology and some of the samples had their glass transition temperatures measured by dynamic scanning calorimetry (DSC). 


\subsubsection{Methyl cellulose}

Methyl cellulose was used as an additive to the particle formulation to model the effects of the incorporation of a cellulose derivative. Concentrations of $2-3 w / v \%$ in the aqueous phase were used. The experimental analysis of the particles that were generated shows the size distribution and morphology has changed from the standard experiment. A high amount of coagulation of the particles, and a large number of small particles surrounding the larger particles was observed (Figure 36).
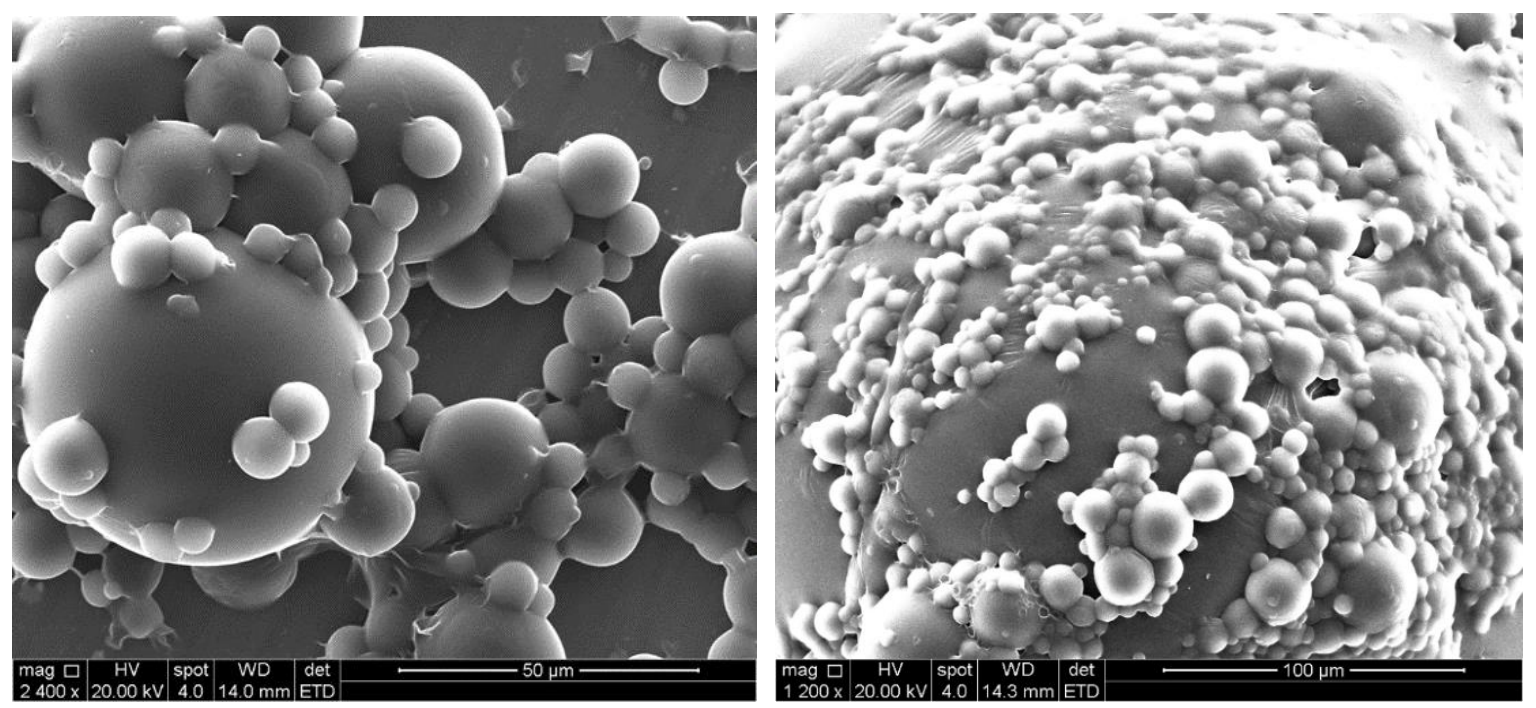

Figure 36: SEM images of particles formulated with methyl cellulose using interfacial polymerisation

The observation of smaller particles surrounding larger particles demonstrates the effects of Ostwald ripening having a significant effect of these samples ${ }^{76}$ It has been shown that the rates of Ostwald ripening in emulsions are highly dependent upon the interfacial tension created by the surfactant. Thus, this behaviour can be attributed to the increased viscosity of the aqueous phase by the addition of the methyl cellulose. This caused the sheer to be applied only to a local area in the reaction vessel, thus causing unreacted starting material to react under little sheer force. Also, with a more viscous aqueous phase, larger particles are expected to form due to diffusion of solvent being reduced, and along with Ostwald ripening having an effect of spreading the particle size distribution.

\subsubsection{Chitosan incorporated particles}

Further mesoparticle preparations were carried out using chitosan as the additive. Early experiments on chitosan were carried out without any modification to the aqueous phase and thus the chitosan was present in solution but as a dispersion. This led to the dispersion being interrupted and resulted in a poor and unstable emulsion with the addition of the organic phase. The following SEM images, of the particles, in Figure 37, show how the disruption to the emulsion affected their morphology. 

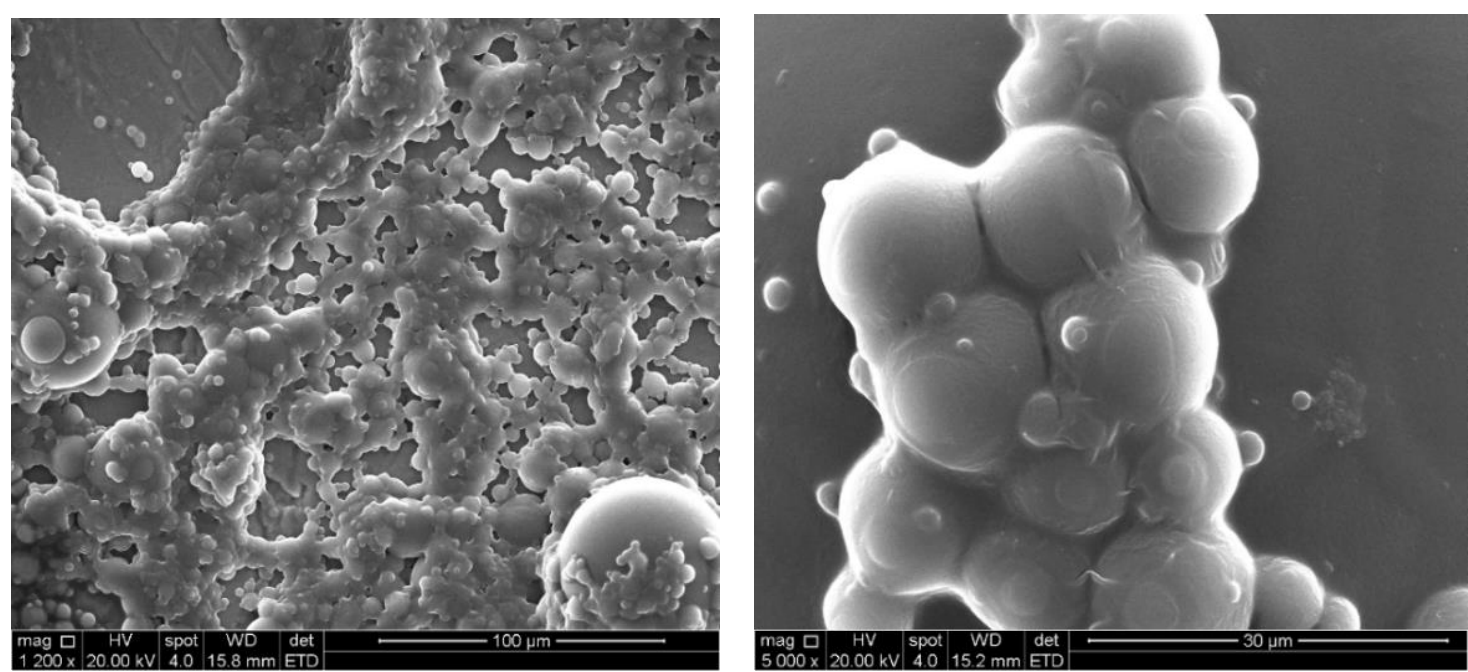

Figure 37: SEM images of particles formulated with chitosan using interfacial polymerisation, without acetic acid (left), with acetic acid (right)

The particles have coagulated much more than the previous experiments. This shows that the presence of an insoluble solid in solution disrupts the emulsion stability, leading to large particle size distributions and non-spherical particle morphologies.

Addition of acetic acid to the aqueous phase effected dissolution of the chitosan. Subsequent experiments were done using a 5-10\% acetic acid aqueous phase, which was found to fully dissolve the chitosan (at $5 \% \mathrm{w} / \mathrm{v}$ ) after a few hours of stirring. These experiments again produced a very viscous aqueous phase that resulted in large particle sizes and evidence of coagulation. The smaller particle with a size of $40 \mu \mathrm{m}$ has an uneven surface, due to the drying of the excesses chitosan onto the particle surface. The shape is irregular with smaller particles bound to the larger ones. This morphology is likely due to the viscosity of the aqueous phase during high sheer, which causes the sheer to be applied only locally in the solution. This leads to a varied concentration of the components throughout the solution, thus formation of particles will vary, leading to the observed particle size distribution (Figure 37)

\subsubsection{Role of polyvinyl acetate (PVA)}

Investigation into the role of PVA on the particle forming reaction was conducted initially using NMR spectroscopy. It was found that the PVA which was in the aqueous phase before reaction was not present in the aqueous fraction after the reaction. Evidence for this is shown in the following NMR spectra, Figure 38. 


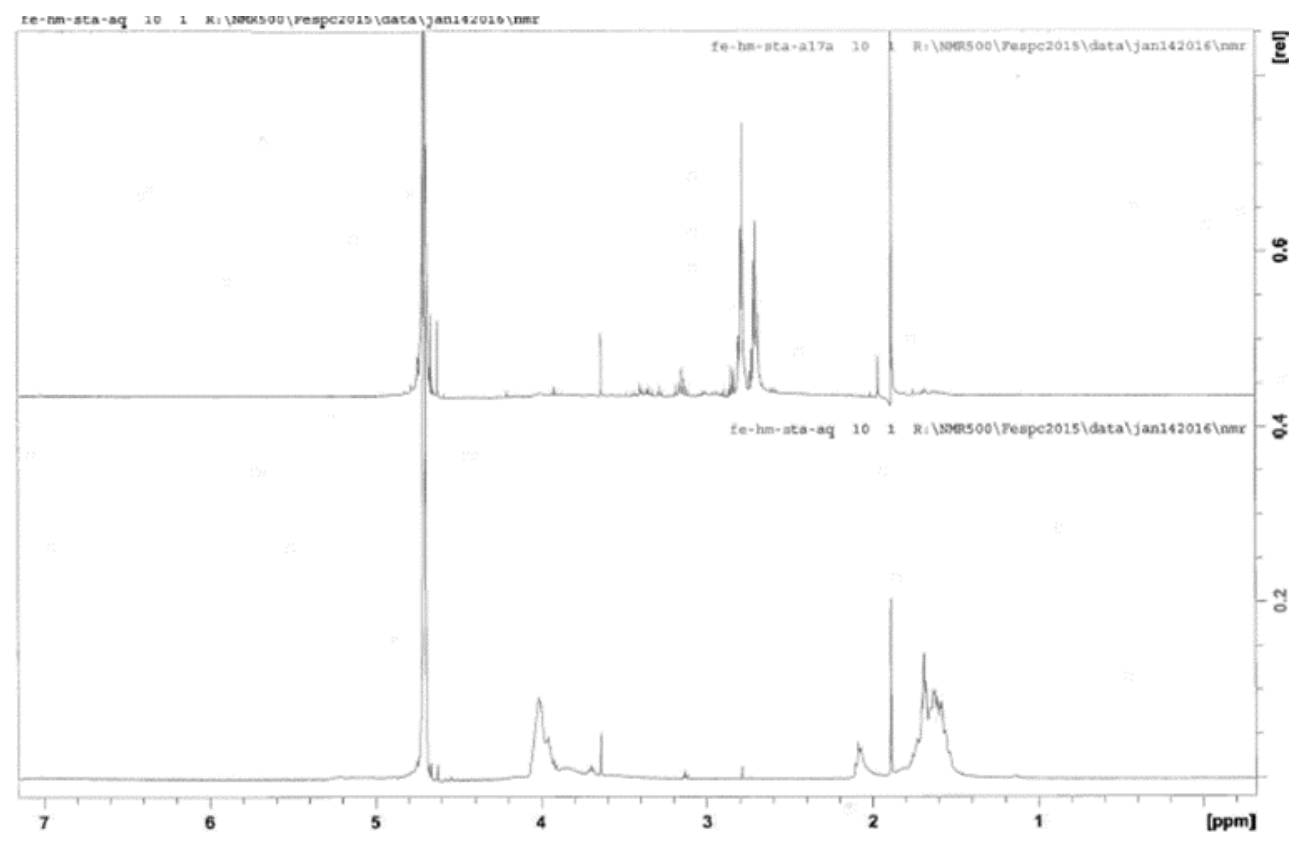

Figure 38: ${ }^{1} \mathrm{H}$ NMR $\left(\mathrm{D}_{2} \mathrm{O}\right)$ of aqueous phase after reaction (top) and aqueous phase before reaction (bottom)

The NMR spectra on top is the aqueous fraction after reaction, and the bottom spectra is the aqueous fraction before reaction. The peaks at 2.1-1.6ppm and 4ppm in the below represent PVA and are the methylene protons and the methine proton respectively. Because there are no peaks attributed to PVA in the spectrum, it is assumed that the majority of the PVA in the aqueous phase has been incorporated into the particles. The PVA is likely incorporated onto the particle surface by surfactant adsorption, which could be occurring during the polymerisation reaction or once the particles have formed. Aqueous PVA is an emulsion; precipitation of particles in emulsions often leads to coagulation and destabilisation of the emulsion.

PVA can be adsorbed onto the surface of polymeric particles and this was proven by the use of X-ray Photoelectron Spectroscopy (XPS). ${ }^{77}$ This spectroscopic method fires X-rays onto a sample which eject core electrons from the atoms, causing a valence electron to take the place of this core electron and release energy as a result. This release of energy is characteristic to the specific atom from which the electron originated, so this can be quantified to give the relative amounts of different atoms in a sample. The method gives information of surface composition, thus was used in this study to find the amounts of PVA on the surface of the samples. They found that some of the polymers tested had higher amounts of adsorbed PVA than others. Polymers such as polylactic acid (PLA) and polylacticco-glycolic acid (PLGA) had higher contributions of PVA, whereas the block co-polymers of PLA or PLGA with polyethylene glycol (PEG) segments showed much lower contributions of PVA. This was attributed to the PEG segments' presence at the water oil interface reducing the requirement for adsorbed PVA to form a stable emulsion. 
Given this result, particle forming experiments were conducted using an aqueous phase but without the presence of PVA. To formulate the aqueous phase without PVA a new aqueous phase formulation was required. In all previous experiments the aqueous phase was a proprietary formulation kindly provided by Lonza, which was made up of $10 \%$ PVA and an unknown quantity of sodium lignosulfonates. The new (non-PVA) phase used a 10\% solution of sodium lignosulfonates in water and this was chosen to replicate the aqueous phase used prior. The lignosulfonates are present to help with the dispersion of the active compounds in the aqueous phase. The initial results of these experiments showed changes to particle morphology and size distribution from those that had additives in the aqueous phase. However, the blank particles (Figure 39) showed little change to morphology, size distributions and averages.
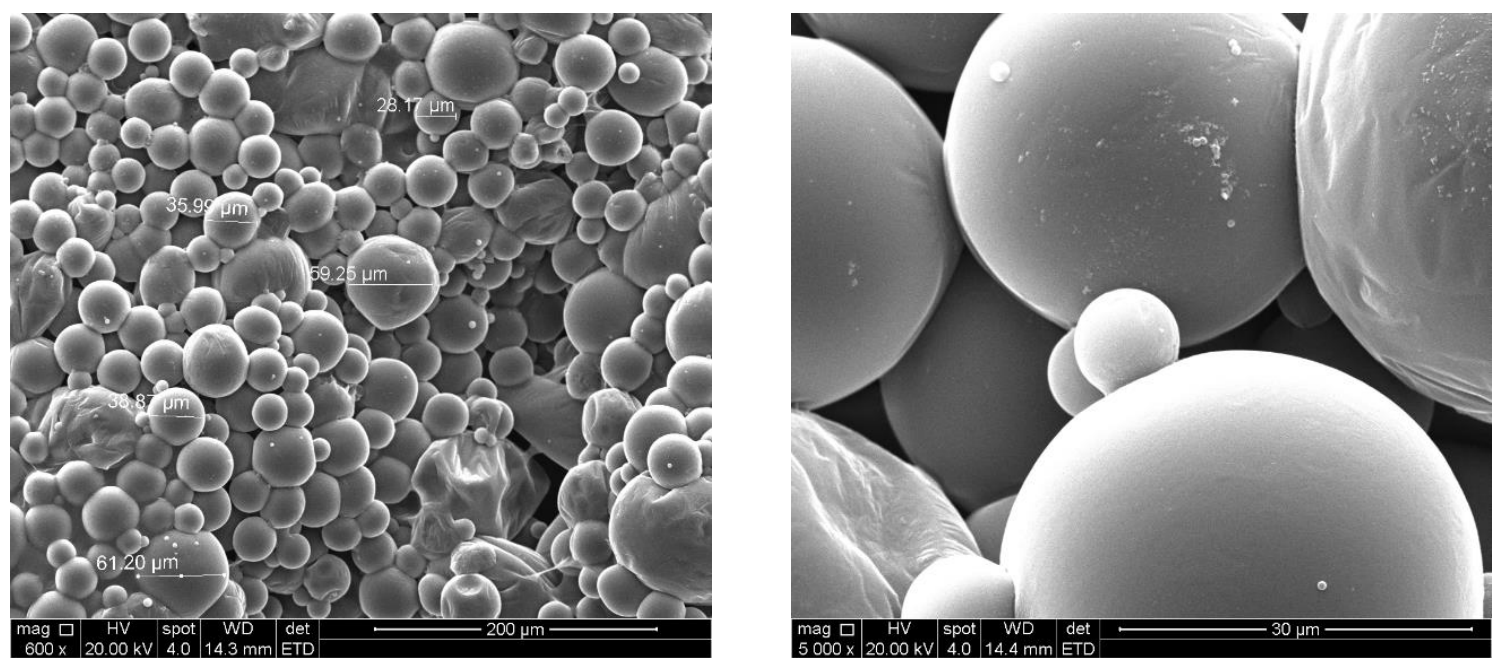

Figure 39: SEM images of particles formulated without PVA, formed using interfacial polymerisation

Particles made without PVA (or insecticide and additives, Figure 39) have smooth, uniform morphology and SEM data indicated a narrow size distribution of $25-60 \mu \mathrm{m}$. The image to the right shows the particles are deforming slightly, likely due to the evaporation of the organic solvent inside the particles when the samples are prepared for SEM. These experiments demonstrated that PVA is not necessary for the formation of particles using interfacial polymerisation, but the stability of the particles is reduced significantly without PVA in the formulation.

\subsubsection{CA incorporated particles}

The final part of this body of work was to incorporate the newly synthesised water soluble cellulose acetates into the polymeric structure of the particles and observe how they affect the system. The first test reaction used approximately $2 \mathrm{w} / \mathrm{v} \%$ of the cellulose acetate (with PVA) and this was first fully dissolved into the aqueous phase before reaction. It was noted when the cellulose acetate was dissolved into the aqueous phase, the viscosity did increase but this was only a subtle change and did not appear to impact particle sizes. The SEM images obtained for this sample do not come from the 
bulk of the sample, the dispersion used for the images was too dilute and did not contain enough particles to give an idea of the bulk morphologies. A repeat experiment was completed using an aqueous phase, this time without PVA. This solution then had the cellulose acetate dissolved into it and the particles were formulated as per the general method. SEM images of these two samples are shown below in Figure 40.
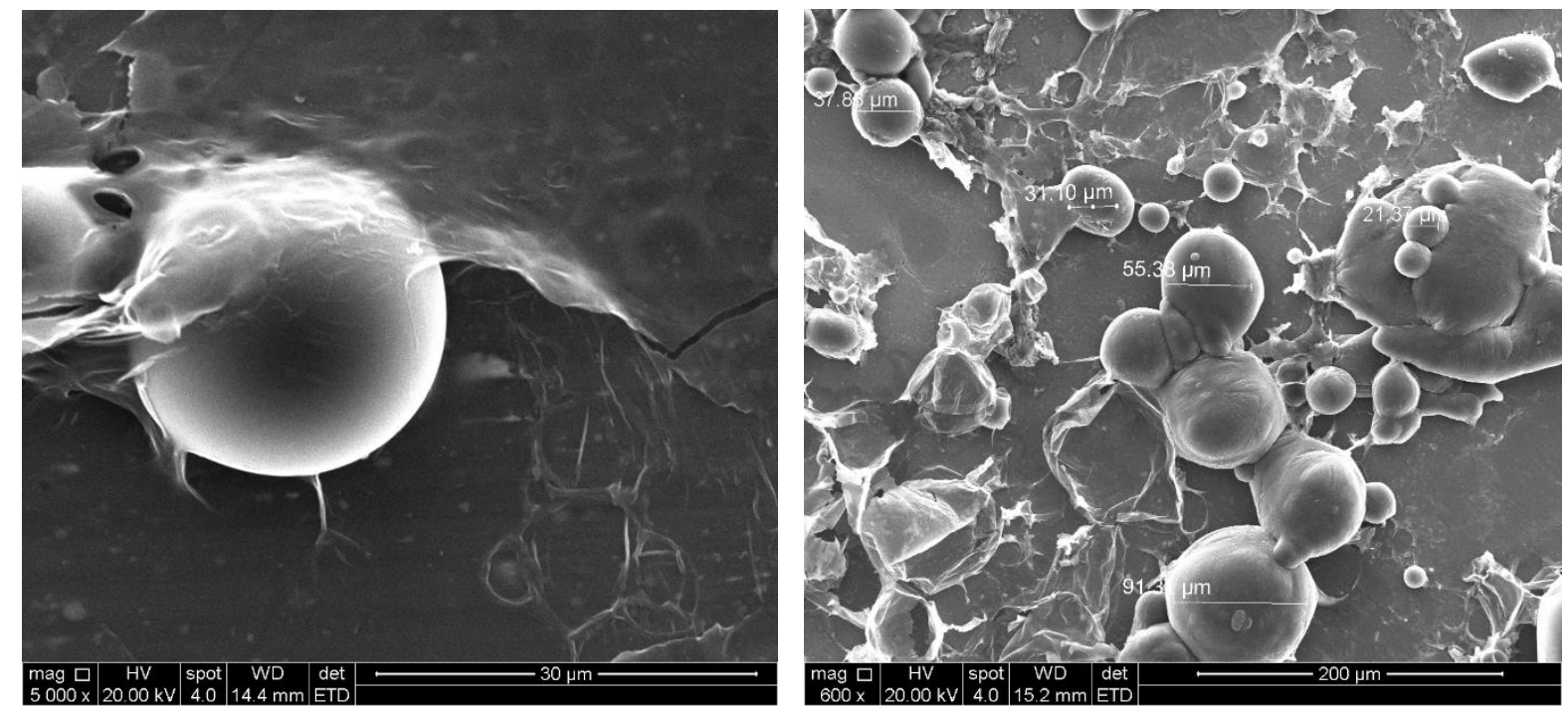

Figure 40: SEM images of particles incorporated with modified cellulose acetate, formed using interfacial polymerisation, with PVA (left), without PVA (right)

The image to the left is the particle formulation carried out with PVA and the image to the right is the formulation without PVA added to the aqueous phase. The 'no PVA' sample showed a high number of destabilised particles, many of which have burst. However, some particles have a smooth and spherical morphology, particularly in the image shown above. The presence of these particles showed promise for this method. However, given that the majority of the particles had a low $T_{g}$ (summarised in Table 3, graphs shown in appendix) and also weak polymeric structures, this process was not investigated further.

Table 3: Glass transition data for samples generated by interfacial polymerisation

\begin{tabular}{lll}
\hline Sample number & Additives & $\mathbf{T}_{\mathbf{g}}\left({ }^{\circ} \mathbf{C}\right)$ \\
\hline $12 b$ & None & 7 \\
$18 \mathrm{a}$ & Cellulose acetate & 7 \\
$16 \mathrm{~b}$ & Methyl cellulose & 19 \\
\hline
\end{tabular}

This body of work showed that cellulose acetates could be incorporated into a hollow polyurea particle. But the particles that were produced were weak and had a low level of incorporation of cellulose derivatives. Uncertainty around whether the wood fungi or termites could digest these 
mixed polymers (that are low in cellulosic materials), drove the decision to not further pursue this process. Future work progressed the production of particles that were made almost entirely of cellulosics and how an active compound could be incorporated into such particles.

\subsection{Double emulsions}

Double emulsions are a very common method used by industry to encapsulate an active compound, where its protection from external elements is essential. Double emulsions can have the phase boundaries as $\mathrm{W} / \mathrm{O} / \mathrm{W}, \mathrm{O} / \mathrm{W} / \mathrm{O}$ or $\mathrm{W} / \mathrm{O} / \mathrm{O}$, with the innermost phase containing the active compound. The diagram below (Figure 41) shows a procedure for the formation of a W/O/O double emulsion.

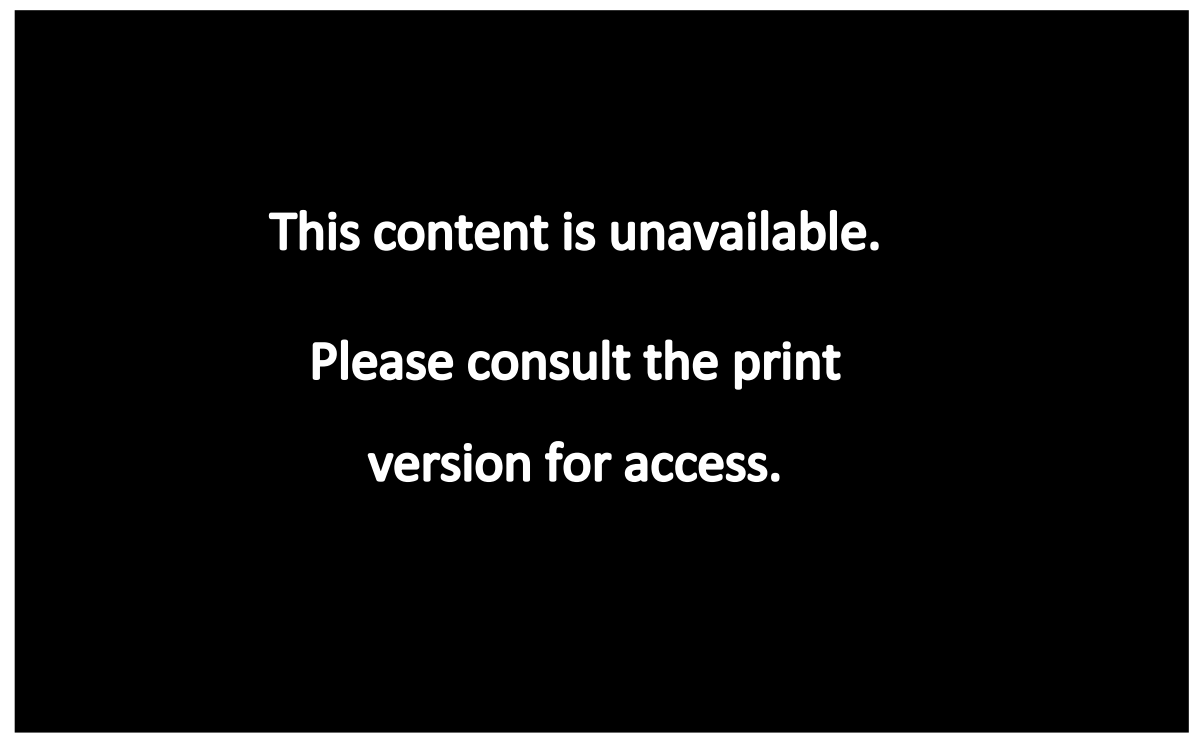

Figure 41: Example diagram of double emulsion procedure ${ }^{41}$

In this project, two different double emulsion techniques were used to attempt to form particles using cellulose derivatives as the encapsulating polymer. Firstly was the 'two drug method', based on work by Sappidi and co-workers. ${ }^{41}$ Here they used ethyl cellulose for the encapsulation of metformin hydrochloride and glipizide. These two drugs were encapsulated between the two phase boundaries of the double emulsion and thus could deliver these two drugs simultaneously in one formulation. It was hypothesised this could be applied to this project by encapsulating both an insecticide and a fungicide into a single formulation. The second method used was based on a microfluidics process carried out in 2003 at the School of Chemical Engineering, Sichuan University. ${ }^{45}$ The method was adapted to a more conventional double emulsion process, as acquiring the necessary equipment was beyond the scope of the project. Only the microfluidics method was analysed by SEM, due to limitations encountered in purification. 


\subsection{1 'Two drug method'}

This method was carried out using traditional multi step double emulsion techniques, the primary emulsion consisted of ethyl cellulose dissolved in a mixed solvent of DMA and acetonitrile (ACN), this organic phase is then emulsified with water. The primary emulsion is then emulsified in a single step with a liquid paraffin solution (including surfactant). $300 \mathrm{mg}$ of ethyl cellulose was dissolved in a 1:1 solution of DMA and ACN, $10 \mathrm{~mL}$ in total was used. This solution was added to $4 \mathrm{~mL}$ of water and this solution mixed at $450 \mathrm{rpm}$ over 15 minutes. This formed the primary emulsion and it was observed to be a cloudy solution with small droplets forming. This solution was then added to a liquid paraffin solution (including $0.15 \mathrm{~mL}$ surfynol $104 \mathrm{E}$ heated to $50^{\circ} \mathrm{C}$ ) and mixed at $750 \mathrm{rpm}$ for 2 hours. The paraffin had to be heated to $50^{\circ} \mathrm{C}$ to become a liquid, as the paraffin used was a soft solid at room temperature. The liquid paraffin solution was extremely viscous, even at the elevated temperature of the solution, leading to the difficulties in purification that were encountered using this method.

The initial experiments using this technique showed the purification of the particles to be the most difficult part of the process. Isolating the particles produced using the method as stated in the literature proved to be difficult to execute. This may have been due to the use of a more viscous and lower boiling point paraffin compared to what was used in the literature. Removal of the paraffin was very difficult. This meant the SEM analysis could not be done on the particles as they could not be effectively isolated from the highly hydrophobic wax. After being unable to isolate the particles using this procedure, it was concluded that the use of liquid paraffin and the purification procedures were too complex for a scale suitable method, and this method was not investigated further.

\subsubsection{Microfluidics}

A method was developed from a literature procedure, ${ }^{45}$ adapting the microfluidic double emulsion process to use conventional double emulsion processes. The adapted method used $1047 \mathrm{mg}$ of ethyl cellulose dissolved in $20 \mathrm{~mL}$ of ethyl acetate along with $0.4 \mathrm{~mL}$ of surfynol $104 \mathrm{E}$. This organic phase is emulsified with the aqueous phase consisting of $40 \mathrm{~mL}$ of water, $1.6 \mathrm{~mL}$ of ethyl acetate and $0.4 \mathrm{~mL}$ of Termul 203. The primary emulsion is then emulsified with another portion of aqueous phase (the same formulation as previously stated) to form a W/O/W emulsion. The polymer was precipitated using $10 \mathrm{~mL}$ of a 1:4 ethanol:water solution and purified using centrifugation. The resulting particles were analysed using SEM and the images are shown in Figure 42. The particles produced using this method were irregular in size and many had collapsed under evaporation (sample preparation). Also, coagulation can be observed which may be due to excess polymer or surfactant in the purified formulation leading to soft readily deformed particles. 

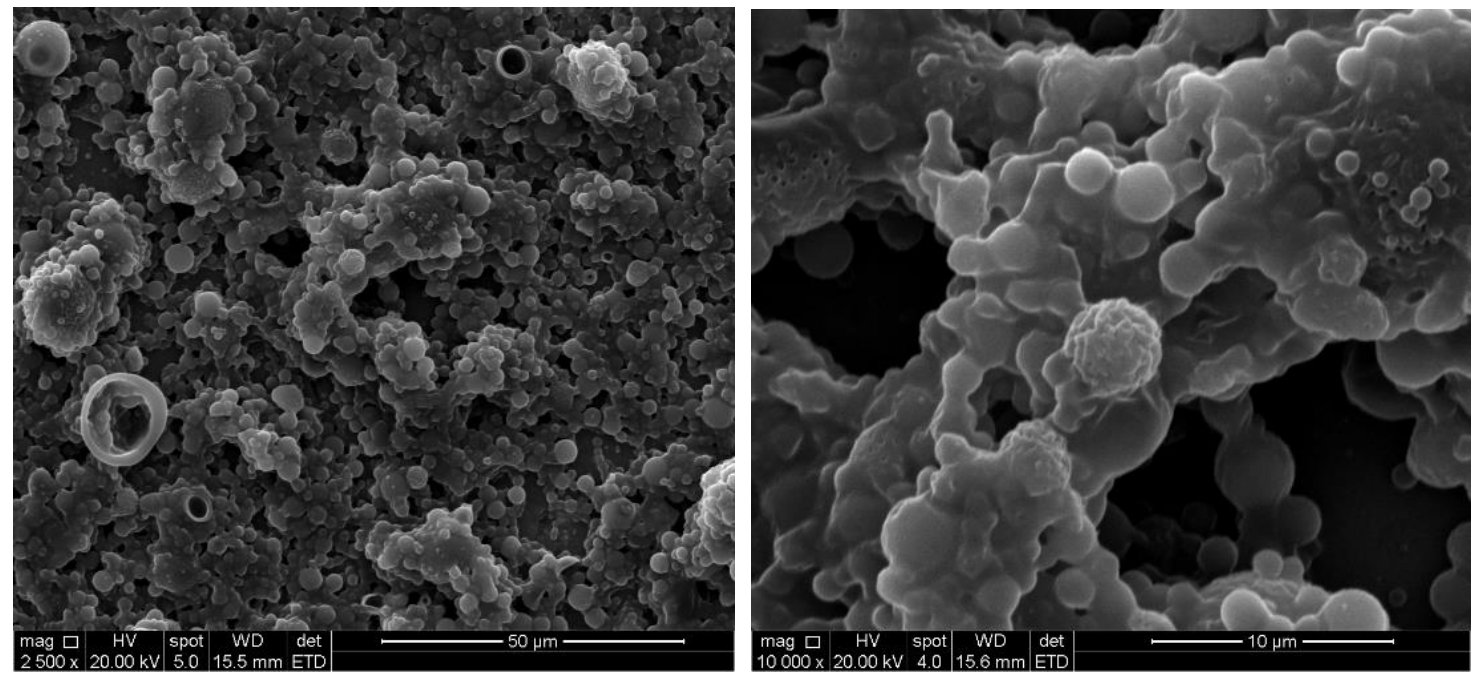

Figure 42: SEM micrographs of double emulsion produced particles for microfluidic method

Given these initial unfavourable results and the complexity of the process, this method was not pursued any further.

\subsection{Solvent diffusion}

The solvent diffusion processes extended the work of Heinze and co-workers ${ }^{49}$. They demonstrated the production of nanoparticles using cellulose derivatives and incorporation of dyes into the polymer matrix. The methods used in this work include emulsification solvent evaporation procedure and low energy solvent displacement (dialysis and controlled precipitation). These methods were tested for their applicability to this project and were analysed by SEM and DLS.

\subsubsection{Dialysis}

This process uses the principle of nanoprecipitation by the slow exchange of solvent through a porous membrane, which allows passive transport of the solvent outside the membrane to be exchanged with the solvent inside the membrane. This is shown diagrammatically in Figure 43. This technique begins with a polymer dissolved in an organic phase, and this solution is then placed into a dialysis membrane and sealed. The sealed bag is placed into a beaker of water, where the aqueous phase slowly diffuses through the membrane and the polymer precipitates very slowly, forming nanoparticles. The process used in this project was adapted from recent work ${ }^{78}$ by Heinze and co-workers. They incorporated various dyes into these nanoparticles, which were made up of cellulose acetate or cellulose acetate phthalate. Their work demonstrated that the dye was incorporated into the particle unevenly throughout its cross-section and that the particles formed were solid, not hollow. This was shown through UV/Vis spectroscopy and SEM. This is an important finding in the context of this project as it suggested that the active compounds may be incorporated throughout the particle unevenly and 
potentially leading to non-uniform leaching characteristics. However, with sufficient small particles in a distribution it was proposed that this undesirable aspect could be overcome in our application of timber treatment. Heinze and co-workers have demonstrated that using acetone as the organic solvent caused the polymer to form non-spherical particle shapes, whereas the use of dimethylacetamide (DMAc) gave spherical particles. ${ }^{48}$ This was observed in various conditions and the reasoning was concluded to be due to the different polarity of the two solvents, interacting with the acetate groups of the cellulose differently and producing these different particle shapes. Because of this finding, DMAc was used exclusively in this body of work.

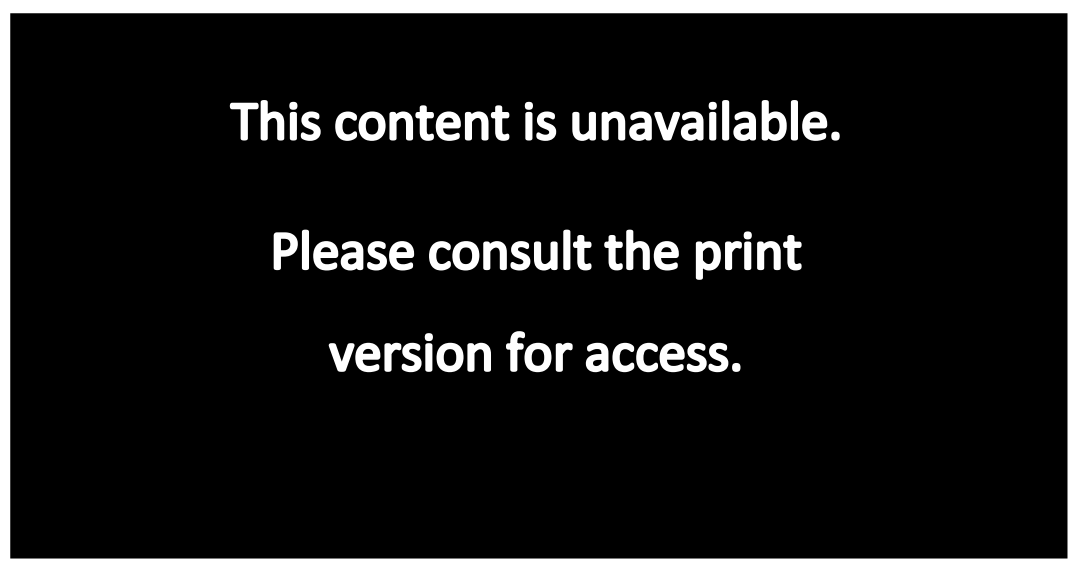

Figure 43: Diagram of particle forming using a dialysis procedure, adapted from ${ }^{79}$

\subsubsection{Initial experiments}

The initial experiments used commercial cellulose acetate (Eastman, CA 398.01) as a particle forming polymer. SEM demonstrates very consistent particle sizes and an average size of $450 \mathrm{~nm}$ was observed under SEM. This was achieved using a polymer concentration of $4 \mathrm{mg} / \mathrm{mL}$ and a solvent exchange time of approximately 15 hours in which the water was replaced after every 3 hours. SEM images for this sample are shown below in Figure 44. This method does not require any additional components such as surfactants or co-solvents, which is an attractive feature of the dialysis method. 

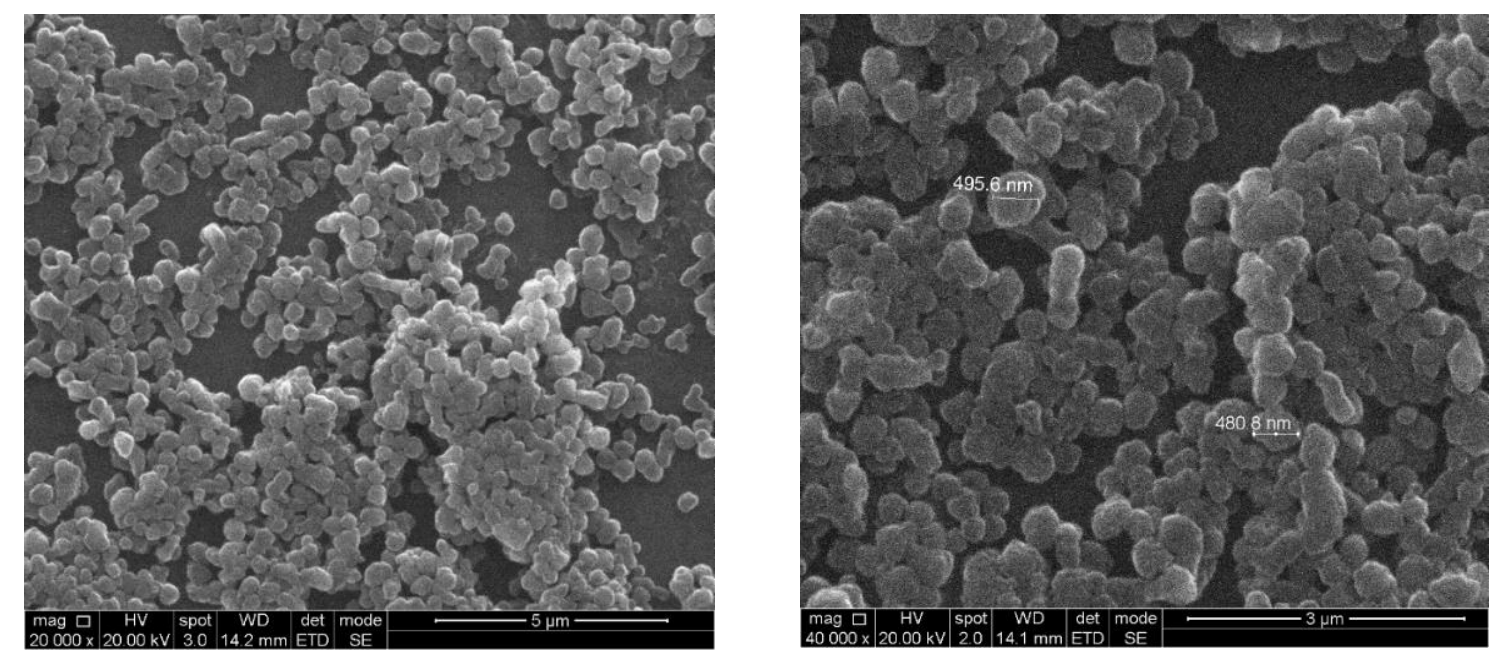

Figure 44: SEM images for particles formed by dialysis method (early experiments)

The particles that were generated have a non-spherical shape and a somewhat rough appearance, and although the particle size distribution appears to be narrow, they do show some evidence of coalescing. This was also observed in the studies carried out by Heinze and co-workers, and they suggested the coalescence and aggregation observed on the SEM was from the drying procedure carried out to prepare the samples for SEM.

The first set of samples produced were isolated with a single centrifugation step and this led to the SEM images showing high coalescence and masked the particles from being observed. An attempt was made to reduce the coalescence observed on the SEM by carrying out further purification steps to remove excess polymer from the suspension. The purification consisted of centrifugation of the suspension and decanting the excess water then re-suspending the particles, with the hypothesis that this process would wash out any contaminants, then the particles would be freeze dried to remove the water. A comparison of the non-purified verses the purified samples can be observed in Figure 46, where the top two samples were not purified, and the rest were purified further.

The particle sizes achieved at a concentration of $4 \mathrm{mg} / \mathrm{mL}$ were slightly small for the scope of this project, for which a size average of $1 \mu \mathrm{m}$ is ideal given the size of the pores in wood. Thus, further work was carried out to manipulate the particle sizes to achieve this target. Carried out in tandem with the particle size manipulation was the incorporation of dyestuff to the particle formulation. The dye tested was Sudan black B (Figure 45), to model the effects of incorporating an active compound in this system, in particular particle size and morphology. ${ }^{78}$ 


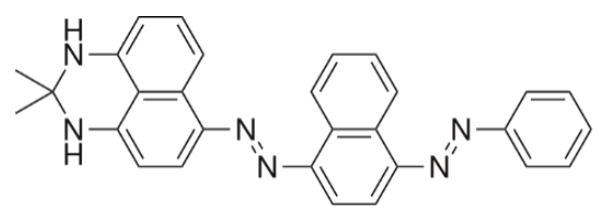

Figure 45: Structure of Sudan black B

With the dye included in the organic phase at a concentration of $1-2 \mathrm{mg} / \mathrm{mL}$, the particles obtained showed very limited morphological changes compared to that of the blank experiments. SEM images of these are shown below in the top two images, however as explained earlier they appear differently to the rest of the samples due to further processing in later samples. The polymer concentrations tested were $4 \mathrm{mg} / \mathrm{mL}, 6 \mathrm{mg} / \mathrm{mL}, 8 \mathrm{mg} / \mathrm{mL}, 12 \mathrm{mg} / \mathrm{mL}$ and $16 \mathrm{mg} / \mathrm{mL}$, and the dye was included only in samples 12a, 12b, 22a and 22b. SEM images acquired for these samples are shown below. Between the 6 samples, the particle sizes that can be observed on the SEM do not change significantly with the changing polymer concentration. This result is puzzling as in the literature they observed an increase in particle sizes with higher polymer concentrations. To further analyse the particle size distributions the use of a dynamic light scattering sizing machine was employed to give a size distribution for the bulk sample. Two different machines were used for this analysis; Zetasizer and a Mastersizer 3000. Each was tested to see which of these would be the most appropriate for this work. 

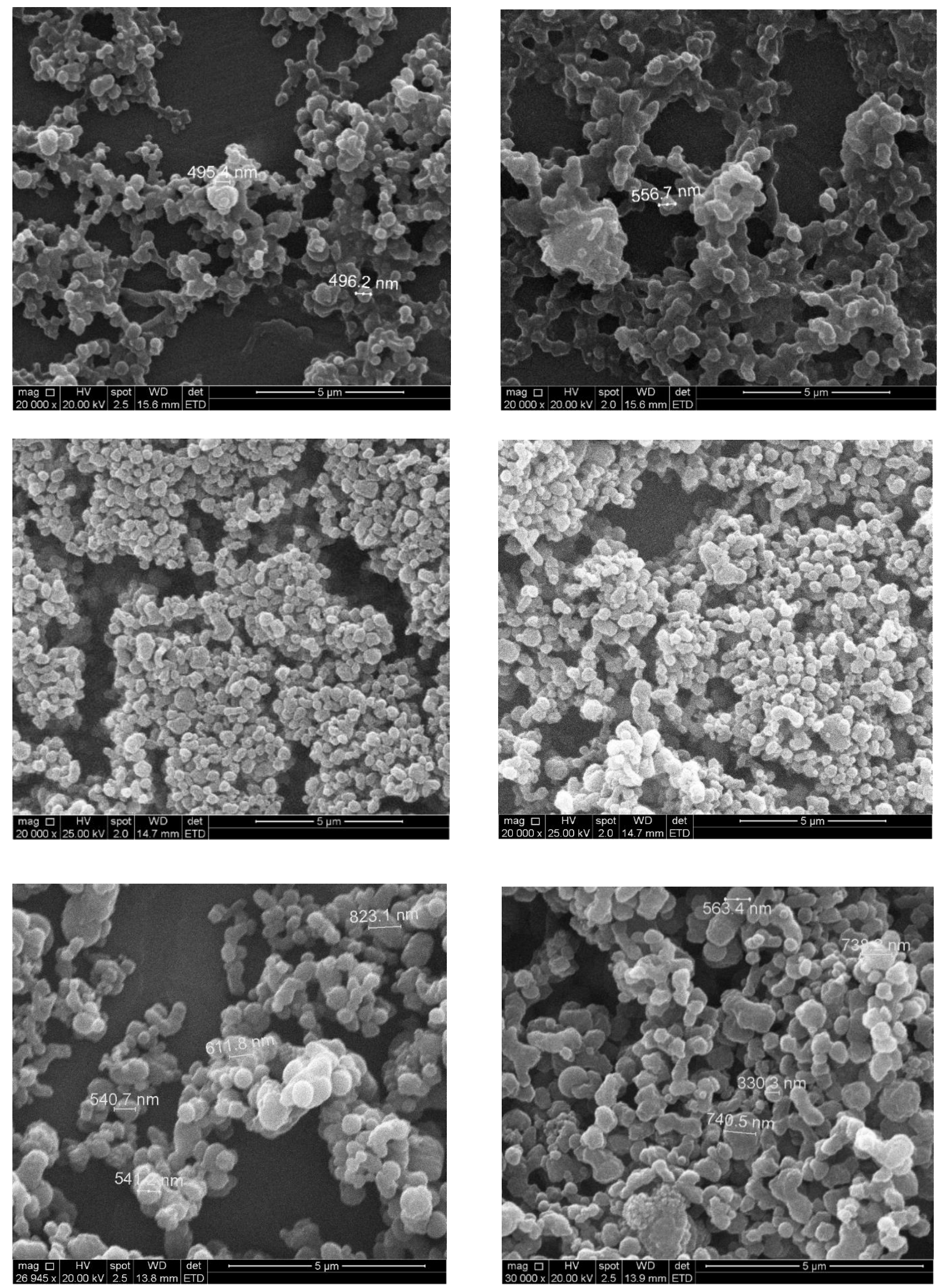

Figure 46: Cellulose acetate nanoparticles produced by dialysis method (Top left: $2 \mathrm{mg} / \mathrm{mL}$, Top right: $4 \mathrm{mg} / \mathrm{mL}$, Middle left: $6 \mathrm{mg} / \mathrm{mL}$, Middle right: $8 \mathrm{mg} / \mathrm{mL}$, Bottom left: $12 \mathrm{mg} / \mathrm{mL}$, Bottom right: $16 \mathrm{mg} / \mathrm{mL}$ ) 


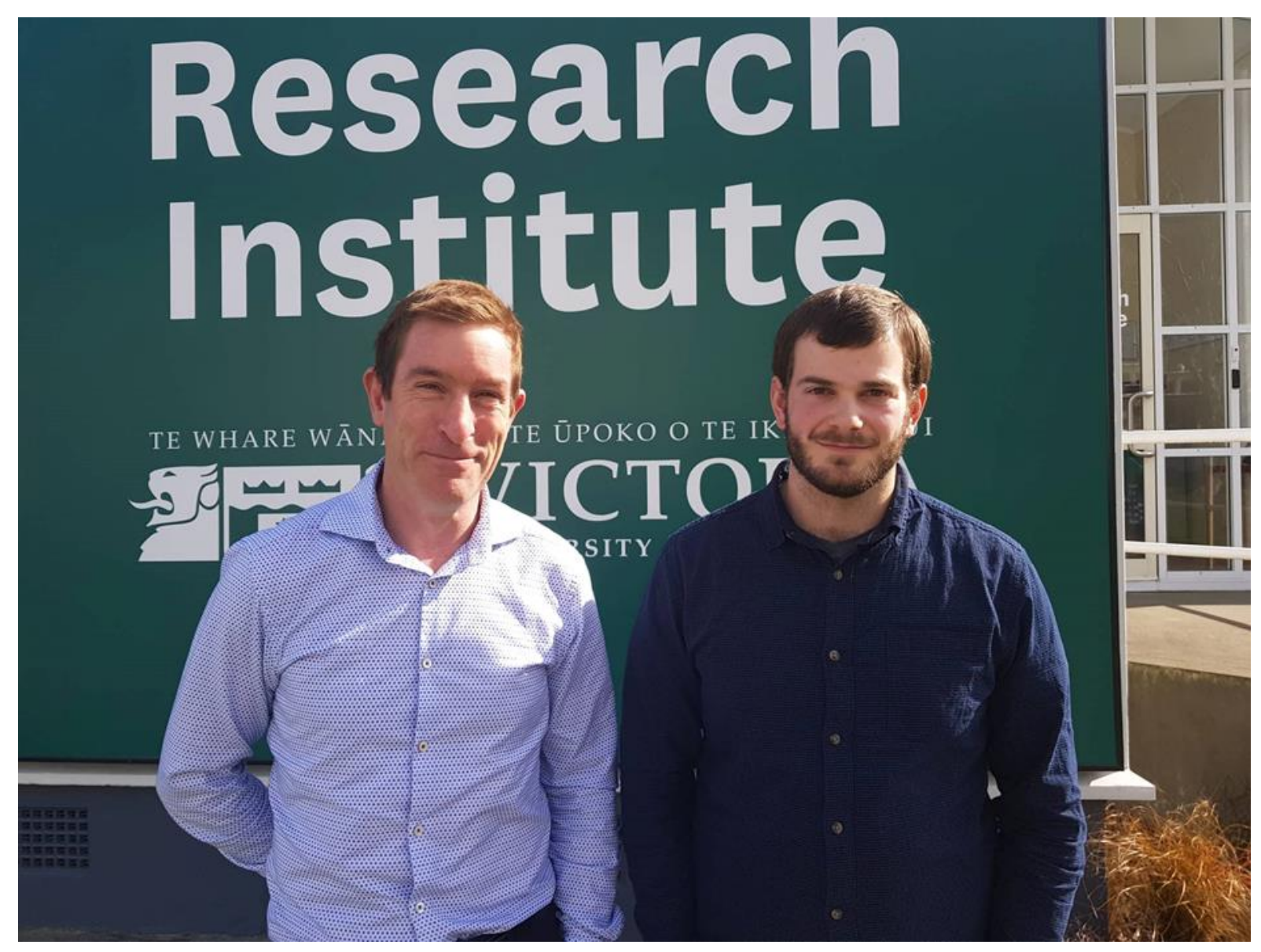




\subsubsection{Particle sizing experiment}

Particle sizing was investigated with both a Zetasizer and a Mastersizer 3000 and the particle size distribution graphs shown are an average of 3 or 5 runs (the repeat analysis was designed to remove any inconsistency in the sampling method). Zetasizer samples are measured in a cuvette (with no stirring or agitation) which is placed in the path of the laser which measures the particle sizes. This machine is designed to use very little sample and is suited to particles on the nanoscale. The first set of three graphs in Figure $\mathbf{4 7}$ show an average particle size of approximately $1 \mu \mathrm{m}$ with very few if any particles below this size. Samples 14a, 22a and 22b (Table 4) were the only samples that were analysed by both the Zetasizer and the Mastersizer. This appears to differ significantly from the particle distribution evidence found through SEM, where most of the samples showed particle sizes of $300 \mathrm{~nm}$ to $700 \mathrm{~nm}$. An interesting point consistent with this data is that SEM images show the particles in a dehydrated state; one would expect the sizes obtained from this to be smaller than those obtained in a hydrated state by light scattering techniques.

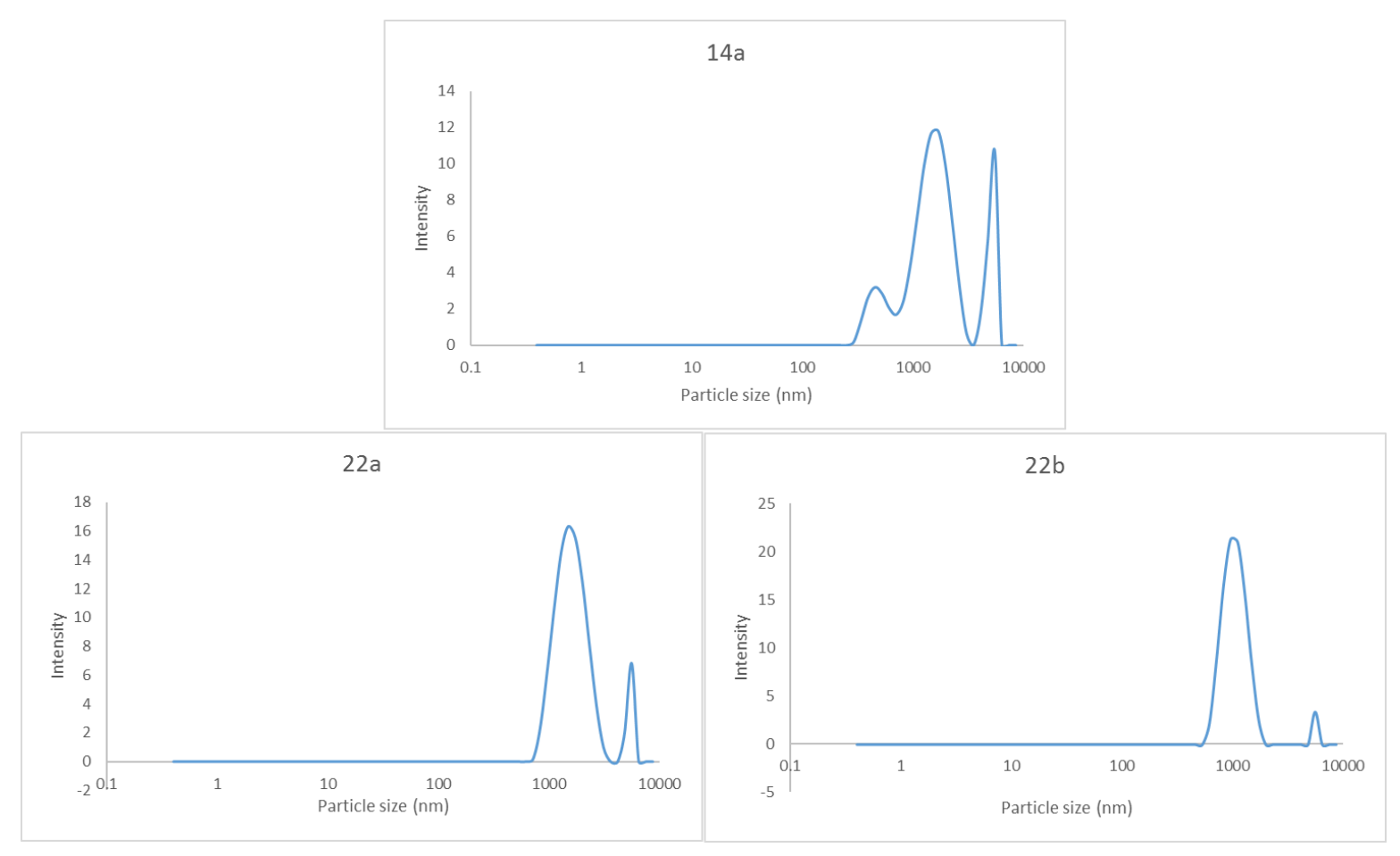

Figure 47: Zetasizer data for samples 14a, 22a and 22b

Aggregation of the particles could also impact the results; therefore, the samples were sonicated for 20 minutes before the measurements were recorded. However, sonication had limited effect on the output attained by the Zetasizer, possibly due to samples not being constantly mixed like they are during Mastersizer experiments, or that aggregation had already occurred in solution. Supporting the aggregation theory was the observation that all three samples showed a large peak at $5.5 \mu \mathrm{m}$ and particles this large could be consistent with the aggregates observed under SEM. There is another 
important point that light scattering is a volume measurement and non-spherical particles could provide artificially high values.

The Zetasizer-data was giving a particle size distribution that less closely matched the evidence from SEM analysis and so was not pursued further. While the data recovered from the Mastersizer (Figure 48) was also not an exact match to SEM observations, it did provide a representation of the smaller particles that were observed only as aggregates in the SEM. It was proposed that this aggregation was occurring during sample preparation for SEM analysis and that the Mastersizer was giving a representation of the smaller particles (albeit at a smaller calculated size than observed by SEM) that were in solution.

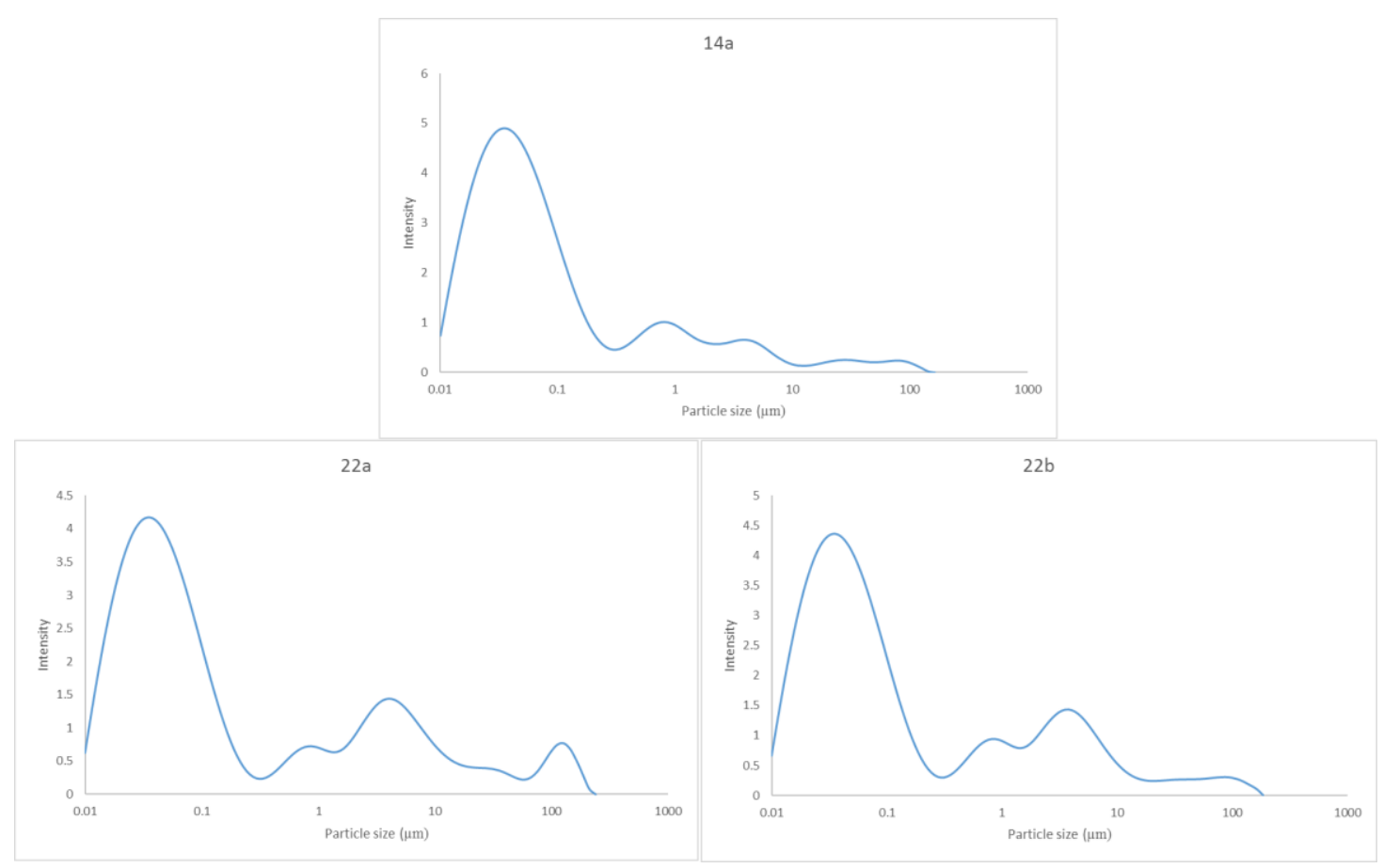

Figure 48: Mastersizer data for samples 14a, 22a and 22b

This assumption was further borne out by observations for particles analysed in later work. For reference compare the SEM data (Figure 49) with that from the Mastersizer data in Figure 51 to that and the Zetasizer spectra (Figure 50).

For particle size comparison between samples, the D-value (D90, D50 and D10) method was used These values show the diameter of which the bulk sample is below the stated percentage; i.e. $90 \%$, $50 \%$, or $10 \%$. For example, the D10 is the diameter at which $10 \%$ of the sample's mass is comprised of particles with a diameter less than this value and so on for $50 \%$ and $90 \%$. This then provides an overall 
picture of the distribution. All samples collected using the Mastersizer will be compared using these values.

The Mastersizer is designed to provide accurate sizing of particles in the $1-100 \mu \mathrm{m}$ range and was used to measure the particle size distributions of all the samples generated throughout this project.

Table 4: D-values for samples generated by dialysis

\begin{tabular}{lllll}
\hline Sample number & Polymer & D10 $(\mu \mathrm{m})$ & $\mathrm{D} 50(\boldsymbol{\mu m})$ & $\mathrm{D90}(\boldsymbol{\mu m})$ \\
\hline $\mathbf{1 4 a}$ & CA-398-3 & 0.019 & 0.057 & 2.03 \\
\hline $\mathbf{2 2 a}$ & CA-398-3 & 0.020 & 0.075 & 12.17 \\
\hline $\mathbf{2 2 b}$ & CA-398-3 & 0.019 & 0.068 & 5.63 \\
\hline
\end{tabular}

\subsubsection{Summary of results}

All the data acquired on the samples produced by the dialysis method are shown in Table 5. It can be concluded that:

- the particle size does not increase significantly with higher polymer concentration

- the polydispersity becomes larger with increasing polymer concentration

- the concentration of dye present has little effect on particle morphology or dispersity.

Table 5: Data acquired for particle forming experiments using the dialysis method

\begin{tabular}{|c|c|c|c|c|c|c|}
\hline $\begin{array}{l}\text { Sample } \\
\text { number }\end{array}$ & Polymer & $\begin{array}{l}\text { Dye conc } \\
\text { (mg/mL) }\end{array}$ & $\begin{array}{l}\text { Polymer } \\
\text { conc } \\
(\mathrm{mg} / \mathrm{mL})\end{array}$ & $\begin{array}{l}\text { Particle size } \\
\text { Range }(\mu \mathrm{m})\end{array}$ & Morphology & Observations \\
\hline $6 a$ & CA-398-3 & N/A & 4 & $0.35-1$ & $\begin{array}{l}\text { Small non-spherical } \\
\text { particles, appear to be solid }\end{array}$ & \\
\hline $12 a$ & CA-398-3 & 1 & 2 & $0.4-0.85$ & $\begin{array}{l}\text { Small non-spherical } \\
\text { particles, appear to be solid }\end{array}$ & \\
\hline $12 b$ & CA-398-3 & 1 & 4 & $0.45-1.1$ & $\begin{array}{l}\text { Small non-spherical } \\
\text { particles, appear to be solid }\end{array}$ & \\
\hline $14 a$ & CA-398-3 & $\mathrm{N} / \mathrm{A}$ & 6 & $0.4-0.75$ & $\begin{array}{l}\text { Small non-spherical } \\
\text { particles, appear to be solid }\end{array}$ & $\begin{array}{l}\text { DLS shows evidence } \\
\text { of agglomeration in } \\
\text { dispersion with } \\
\text { water }\end{array}$ \\
\hline $14 b$ & CA-398-3 & N/A & 8 & $0.4-1.9$ & $\begin{array}{l}\text { Small non-spherical } \\
\text { particles, appear to be solid }\end{array}$ & \\
\hline $16 a$ & CA-320-S & 2 & 2 & $0.5-1.9$ & $\begin{array}{l}\text { Small spherical particles } \\
\text { with a smooth surface }\end{array}$ & $\begin{array}{l}\text { Particles appear to } \\
\text { be coalescing }\end{array}$ \\
\hline $17 a$ & CA-320-S & 2 & 12 & $0.7-1.4$ & $\begin{array}{l}\text { Small spherical particles } \\
\text { with a smooth surface }\end{array}$ & \\
\hline $22 a$ & CA-398-3 & 2 & 12 & $0.4-0.8$ & $\begin{array}{l}\text { Particle size more } \\
\text { polydispersed than } 14 a\end{array}$ & $\begin{array}{l}\text { SEM shows } \\
\text { evidence of } \\
\text { unknown particle } \\
\text { with different } \\
\text { morphology }\end{array}$ \\
\hline $22 b$ & CA-398-3 & 2 & 16 & $0.35-0.93$ & $\begin{array}{l}\text { Particle size more } \\
\text { polydispersed than } 14 a\end{array}$ & \\
\hline
\end{tabular}


The inconsistent results attained by the dialysis method proved difficult to explain. The two DLS machines used gave conflicting data in comparison to the SEM evidence. This was put down to the process generating irregular particles that would aggregate far too easily under normal conditions. Without the aid of surfactants to prevent aggregation this process was deemed to be inappropriate for the project. The success of other methods being tested meant developing this method further was unnecessary. An attempt was made to include a surfactant in the organic phase to prevent excess agglomeration of the particles but was not successful. The inclusion of surfactants in this process produced particles that could not be purified successfully due to the strength of the surfactant used (Maxemul 7101), thus this method was no longer pursued.

\subsection{Phase inversion}

The technique of phase inversion is a very common industrial method of producing particles where low polydispersity is required. This method was applied in conjunction with 'salting out' to produce cellulose derivative particles with the best morphology and size distributions attained by any of the methods used to date. The term 'salting out' refers to the process of utilising solutions containing high ionic strength to change the effective solubility of a non-electrolyte in an aqueous solution.

The method required dissolving ethyl cellulose into acetone along with the active ingredient, then preparing an aqueous phase with polyvinyl acetate and calcium chloride dissolved into it. ${ }^{60}$ The high ionic strength of the aqueous phase makes it immiscible with the acetone, so an emulsion can be formed between these two phases. Once the emulsion has been formed, excess water is added which forces the polymer to precipitate by changing the solubility profile of the aqueous phase. In our work we required the particles to have a size average of approximately $1 \mu \mathrm{m}$ and for the particles to be robust under harsh conditions. As such, the method was adapted to use cellulose acetate butyrate (CAB) in place of the ethyl cellulose, as its physical properties better suited the project requirements. Ethyl cellulose polymer is better for drug delivery as it is more flexible when formed into a film and is more porous. ${ }^{13}$ The CABs form much more durable and stronger films in comparison, which was desired for this project due to the harsh environment the particles will have to endure during the wood processing procedures.

The initial experiments were conducted to compare the difference of particles generated using ethyl cellulose and CABs. Further experiments then tested the different commercially available CABs along with different polymer concentrations. A model active ingredient was incorporated to test the robustness of the process. The particle size was then optimised by manipulation of several key factors; addition time of the aqueous phase and surfactant concentration. Finally, incorporation of the fungicides into the formulation and the analysis of encapsulation efficiencies, was undertaken. 


\subsubsection{Initial experiments}

Preliminary experiments used ethyl cellulose (EC-10) as the polymer, and a separate set of experiments used CAB 381.01 as the polymer; both sets of experiments were done without any active compounds present. The concentration of the polymer was kept at $25 \mathrm{mg} / \mathrm{mL}$ for both the ethyl cellulose and $C A B$ experiments. The surfactant used was PVA at a concentration of $3 w / w \%$ and the salt used was calcium chloride at $20 \mathrm{w} / \mathrm{w} \%$. The resulting particles from these experiments were consistent with what was obtained from the literature and the morphologies were consistently smooth and spherical (Figure 49).
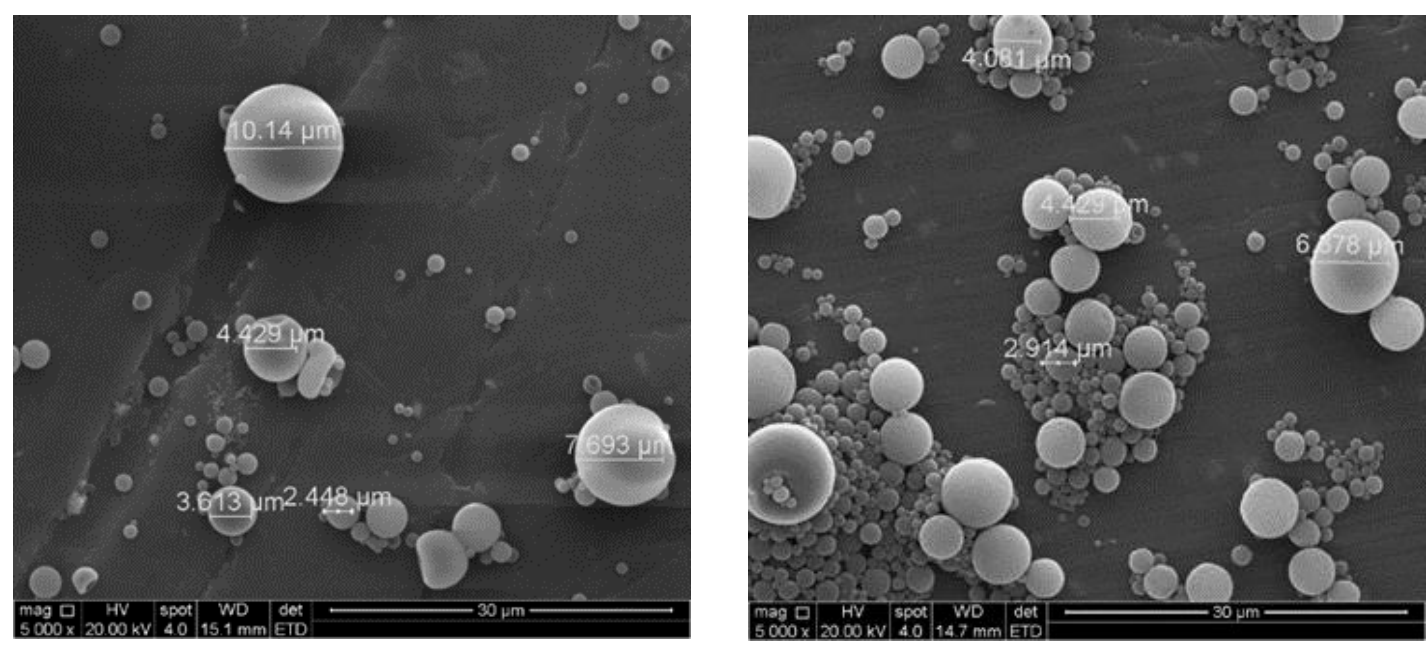

Figure 49: SEM micrographs, EC-10 particles (left), CAB 381.01 particles (right)

The particles produced using ethyl cellulose had a very smooth surface and were regular spheres, which was also true of the particles produced using cellulose acetate butyrate. Also, particle size distributions of the two samples using the different cellulosic polymers were very similar (Figure 50).

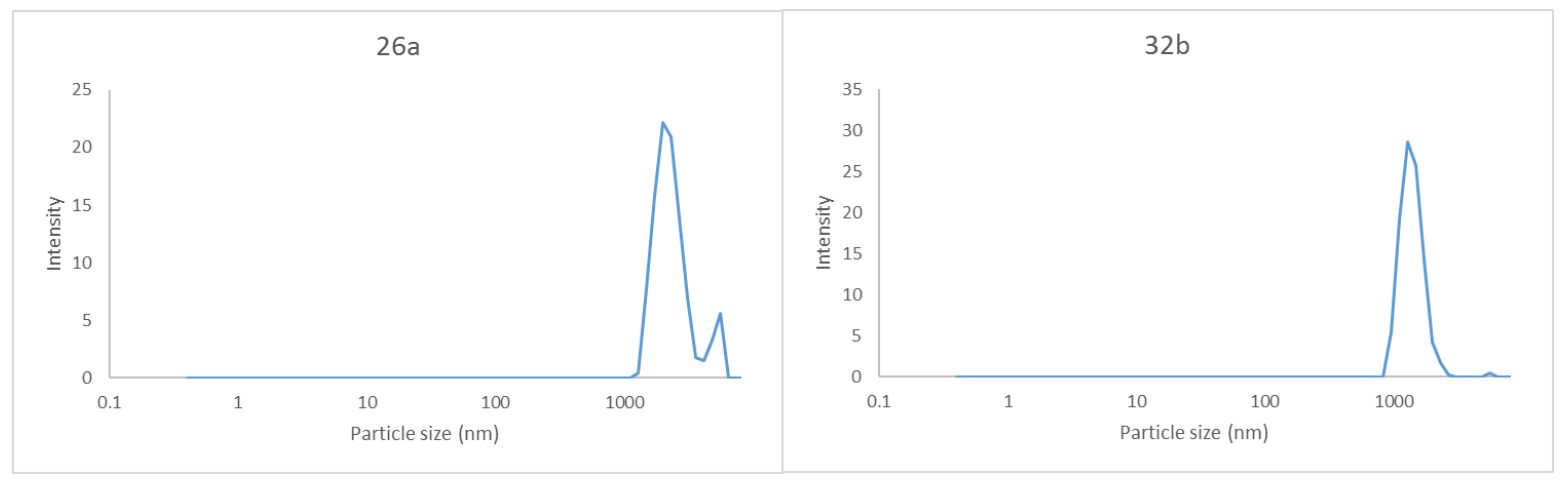

Figure 50: DLS data, EC-10 particles (left), CAB 381.01 particles (right), Zetasizer

The graphs above show the two samples have almost identical size distributions, and along with the similar morphologies, the change of polymer has had little effect on the formation of particles. It was decided in this case to also collect DLS data with the Zetasizer just to confirm whether discrete smooth 
particles (as seen in the SEM image) would give particle size data that better correlated between the techniques of SEM and the Zetasizer. Repeat analysis gave highly reproducible data. However, when the samples were run on the Mastersizer, larger particle sizes above $3 \mu \mathrm{m}$ were detected that appeared to be absent at the same level as in the Zetasizer data. To compare the data-sets for the phase inversion samples, a particle size distribution graph collected from sample 32a using the Mastersizer is shown in Figure 51. Sample 26a was not analysed using the Mastersizer.

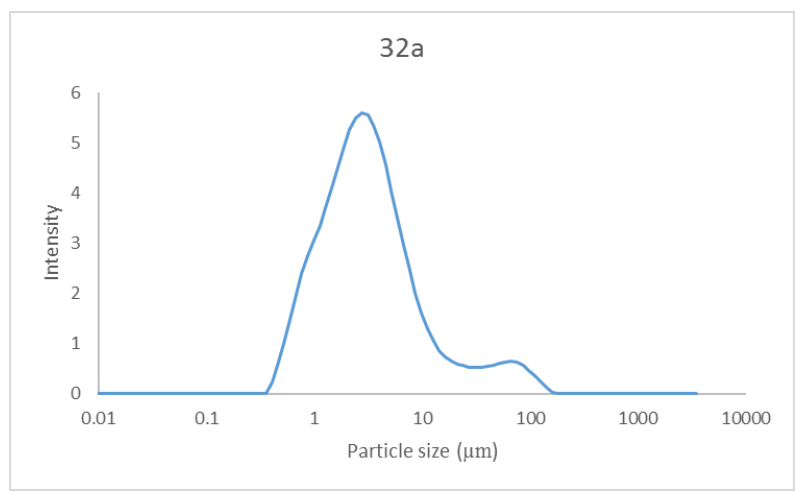

Figure 51: DLS data of CAB 381.01 particles using Mastersizer

The graph above shows the peak particle size is approximately $5 \mu \mathrm{m}$, but importantly, a population of smaller particles $<1$ um are present as a shoulder in the distribution. This is consistent with the observation of a distribution of small particles in the SEM image (Figure 49). Also DLS data details a small population of larger particles - again consistent with the observation of larger particles around $50 \mu \mathrm{m}$ which can be observed on the SEM. In these initial experiments, CAB 381.01 was used due to its low viscosity, and the polymer concentration was not varied. Given the consistent results obtained from this method and the robustness of the process this was the primary method used in this project. Further experiments were then carried out on the different commercially available CABs along with testing different polymer concentrations to observe their effects on the system.

\subsubsection{Polymer testing}

The polymers tested were all commercially available CABs; the difference between them is the degree of substitution of the different functional groups (hydroxyl, acetate and butyrate). This has the effect of changing the physical properties of the polymer including viscosity, solubility and glass transition temperature. Of importance to the phase inversion process is the viscosity of the organic phase. The effect viscosity has on the organic phases, due to the polymer, was determined by testing the different CABs (Table 6) while using the same concentrations. With increasing concentration of the polymer, the expected outcome would be an increase in particle size. This is because of the combination of the higher viscosity associated with higher polymer concentrations, and the slower diffusion of solvent 
through the organic phase, leading to larger particles being generated. Thus, the concentration was varied to observe the effect this had on the phase inversion system.

Table 6: Physical properties of commercially available cellulose acetate butyrate

\begin{tabular}{llllll}
\hline Polymer & Viscosity (poise) & Hydroxyl \% & Acetate \% & Butyrate wt \% & $\mathrm{Tg}_{\mathrm{g}}\left({ }^{\circ} \mathrm{C}\right)$ \\
\hline CAB 381.01 & 0.38 & 1.5 & 13 & 51 & 123 \\
CAB 553.04 & 1.14 & 4.8 & 2 & 46 & 136 \\
CAB 171.01 & 57.37 & 1.5 & 29.3 & 17 & 161 \\
CAB 500.5 & 19 & 1 & 4 & 51 & 96 \\
CAB 551.01 & 0.038 & 1.5 & 2 & 53 & 85 \\
\hline
\end{tabular}

Each of the four polymers were tested using four different concentrations; $25 \mathrm{mg} / \mathrm{mL}, 50 \mathrm{mg} / \mathrm{mL}$, $75 \mathrm{mg} / \mathrm{mL}$ and $100 \mathrm{mg} / \mathrm{mL}$. With concentrations higher than $75 \mathrm{mg} / \mathrm{mL}$, there was a small observed increase in average particle size. However, this was not the case for all the polymers and was not reproducible with subsequent experiments. Experiments with the polymer CAB 381.01 gave the best results; with the increasing polymer concentrations, higher average particle sizes and an increase in polydispersity were observed (Figure 51).

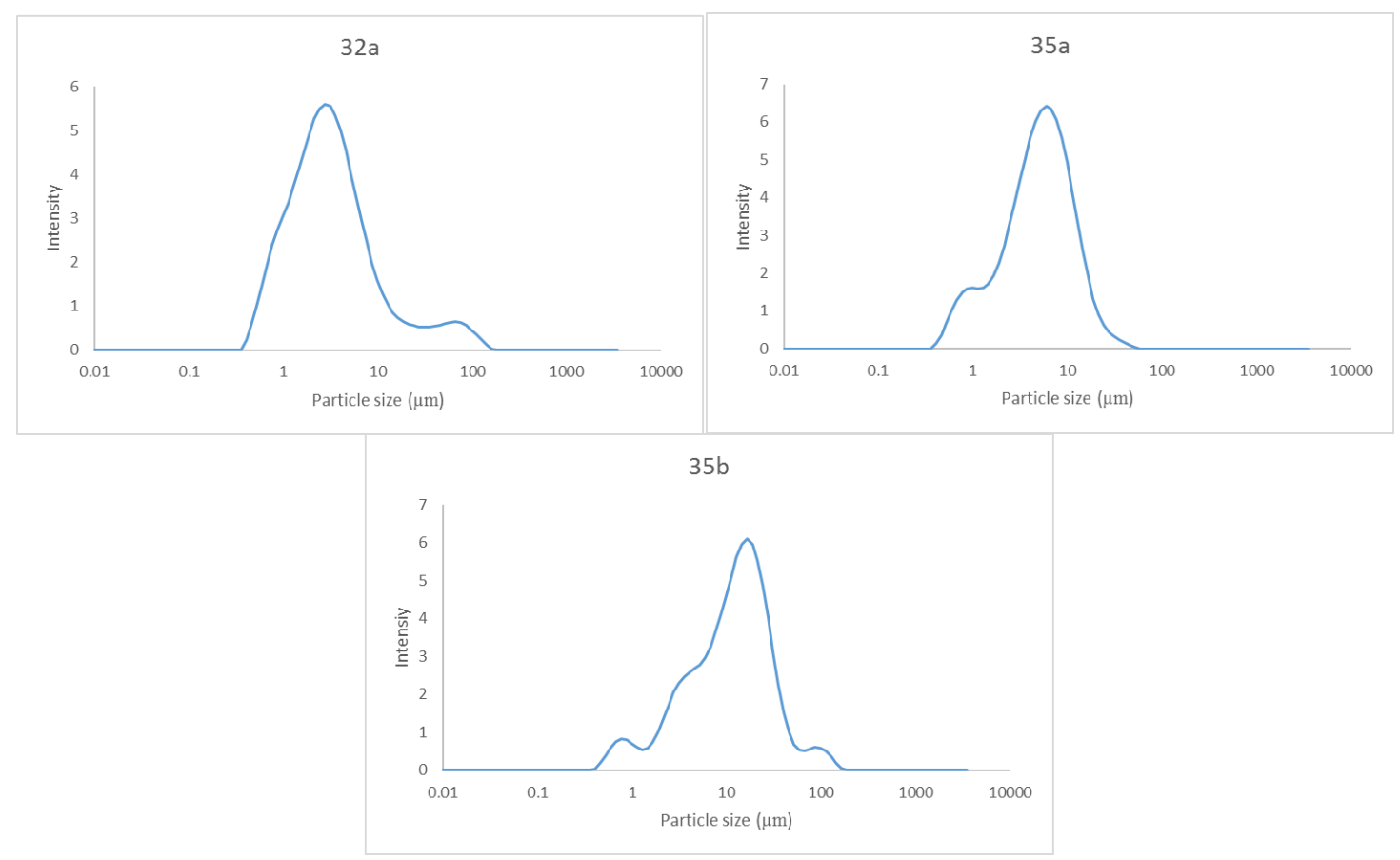

Figure 51: DLS data for CAB 381.01, 25mg/mL (top left), $50 \mathrm{mg} / \mathrm{mL}$ (top right), $75 \mathrm{mg} / \mathrm{mL}$ (bottom)

The bulk distribution moves to larger particle sizes with each increase in polymer concentration, as well as the overall distribution getting larger. The D-values (Table 7) clearly show this change in particle size, as the D90 values go from $12.7 \mu \mathrm{m}$ to $34.4 \mu \mathrm{m}$, as we move from sample $35 \mathrm{a}$ to $35 \mathrm{~b}$. Also, 
the D10 and D50 values increase in a similar amount for the same samples, this indicated the whole distribution has moved to higher particle sizes, not just a wider distribution. This data is consistent with how increases in viscosity affect the particle size distribution and average size. This pattern was also observed with some of the different polymers shown above, particularly $C A B 553.04$ and $C A B$ 551.01. However, the higher viscosity polymers, $C A B 171.01$ and $C A B 500.5$, gave inconsistent particle size distributions and morphologies. This was likely due to the high organic phase viscosities of the experiments, with these polymers leading to more localised mixing when the sheer was applied to the system (Figure 52).
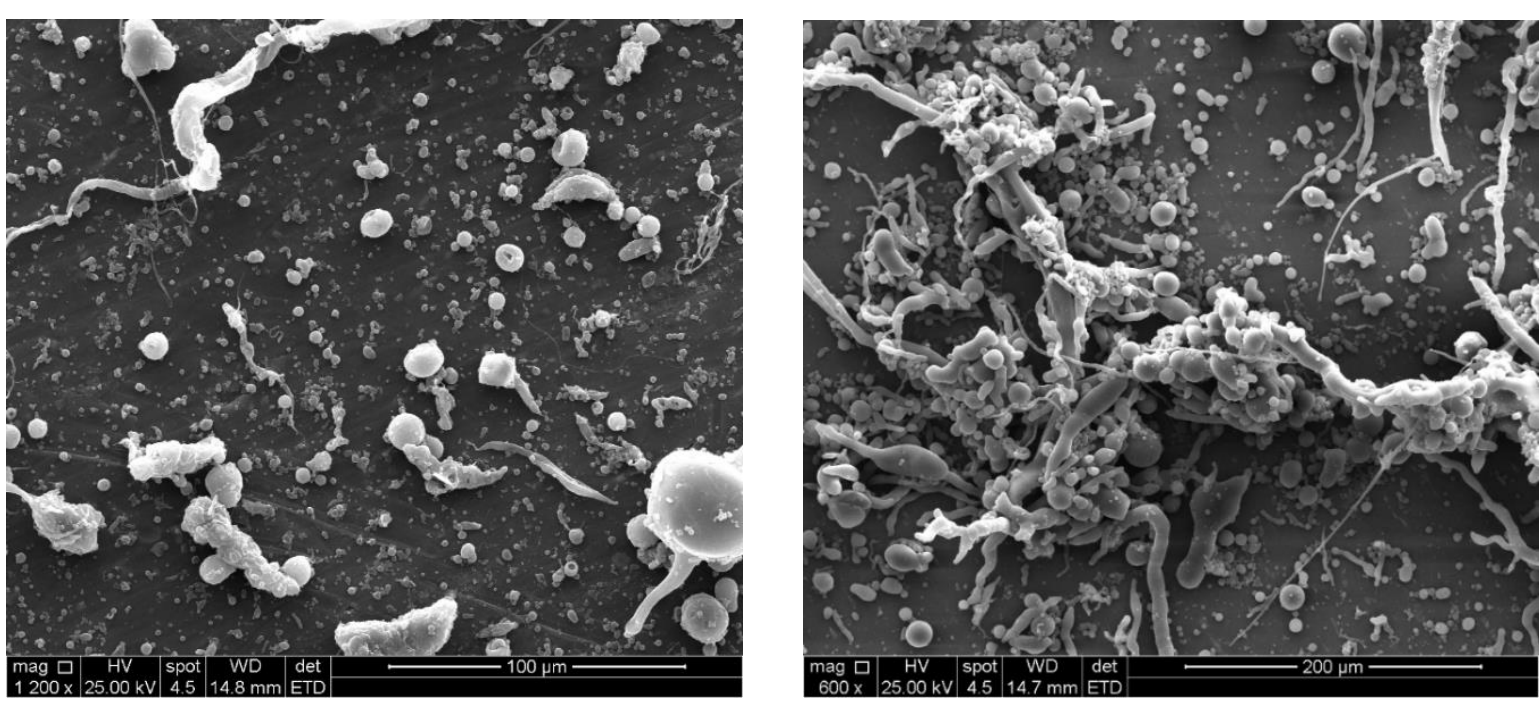

Figure 52: SEM images of sample 42d (CAB 171.01, left) and 41a (CAB 500.5, right)

The samples shown above are the second set of samples generated using these high viscosity polymers, which generated irregular structures with only some spherical particles. This demonstrated that these high viscosity polymers were unsuitable for reproducible particle manufacture. Polymer CAB 551.01 gave good results, however; the DLS data showed a much smaller change to the particle size distribution (Figure 53). 

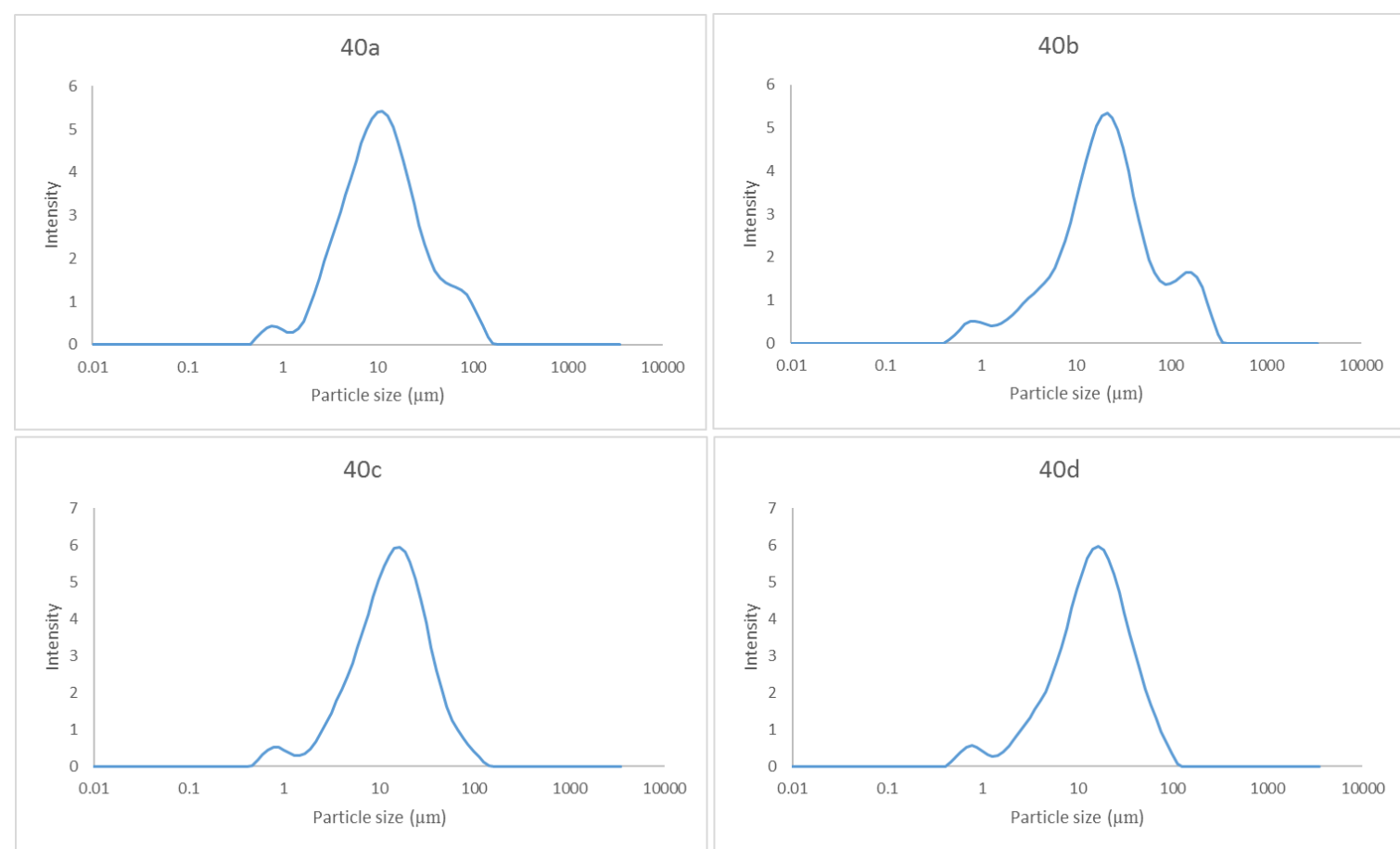

Figure 53: Mastersizer data for samples 40a-d

The D-values for samples 40a-d showed only small variations to the size distribution, with the D90 of $40 \mathrm{~b}$ giving the highest value of $120.1 \mu \mathrm{m}$, indicating this sample has larger particles on average. The graphed data displayed a shoulder on the main peak, indicating some Ostwald ripening may be occurring here (sample 40b, Figure 54). The higher concentration samples (40c and 40d) showed little difference in the D90 value $(41.7 \mu \mathrm{m}$ compared to $44.9 \mu \mathrm{m})$ and this was also consistent with the sample prepared with the lowest concentration of polymer (40a; $47.2 \mu \mathrm{m})$. This evidence showed there to be no significant change to the bulk size distribution with increasing concentration of polymer, which differs from the results attained by polymer $C A B$ 381.01. However, polymer $C A B$ 381.01 did yield an overall smaller particle range, indicated by their lower D10 values $(1.3 \mu \mathrm{m}$ and 2.6 $\mu \mathrm{m}$ respectively) than that produced by polymer CAB $551.01(3.15 \mu \mathrm{m}$ and $4.3 \mu \mathrm{m})$, which indicated a higher population of particles in the $1 \mu \mathrm{m}$ range for samples prepared using $C A B 381.01$. 


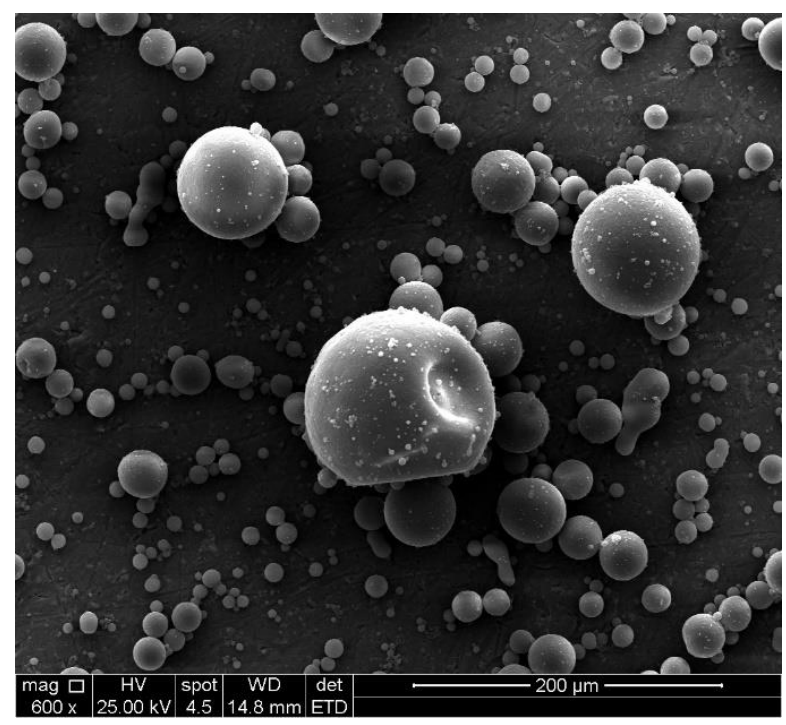

Figure 54: SEM image of sample 40b, demonstrating the larger particles present

The results of testing different polymers have demonstrated that the polymer concentration effected the particle size distribution. Two of the polymers (CAB 553.04 and CAB 500.5) proved to be too viscous for the project application and gave non-spherical particles as a result. The polymers $C A B$ 381.01 and $C A B 500.5$ showed the most consistent results, however only CAB 381.01 showed the trend of increasing particle size with higher polymer concentrations. Thus, CAB 381.01 was chosen as the lead polymer for continued examination due to its more reproducible behaviour, spherical particle formation and desirable particle size.

Table 7: D-values for samples produced using different CAB polymers

\begin{tabular}{llllll}
\hline $\begin{array}{l}\text { Sample } \\
\text { number }\end{array}$ & Polymer & $\begin{array}{l}\text { Polymer } \\
\text { concentration } \\
(\mathbf{m g} / \mathbf{m L})\end{array}$ & D10 $(\boldsymbol{\mu m})$ & D50 $(\boldsymbol{\mu m})$ & D90 $(\boldsymbol{\mu m})$ \\
\hline 32a & CAB 381.01 & 25 & N/A & N/A & N/A \\
\hline 35a & CAB 381.01 & 50 & 1.29 & 5.40 & 12.74 \\
\hline 35b & CAB 381.01 & 75 & 2.59 & 12.90 & 34.44 \\
\hline 40a & CAB 551.01 & 12.5 & 3.16 & 11.48 & 47.29 \\
\hline 40b & CAB 551.01 & 25 & 4.33 & 21.87 & 120.11 \\
\hline 40c & CAB 551.01 & 50 & 3.98 & 14.96 & 41.70 \\
\hline 40d & CAB 551.01 & 75 & 3.92 & 15.68 & 44.94 \\
\hline
\end{tabular}




\subsubsection{Particle size optimisation}

To optimise the particle size using the phase inversion method, two parameters of the method were changed; the addition rate of the aqueous phase and the surfactant concentration. ${ }^{57}$ It has been reported that increasing the time taken to add the aqueous phase along with higher surfactant concentrations leads to smaller particles and narrower size distributions. This was attributed to how the longer addition time changed the type of phase inversion occurring in the system from a catastrophic to a transitional phase inversion. As discussed in the introduction, catastrophic phase inversion will generate larger particles sizes and have wider size distributions, whereas the opposite is true in both cases for transitional phase inversion. It was reported ${ }^{57}$ that significant particle size distribution changes could be affected by changes in addition times from 60 to 105 seconds and by modifying the surfactant concentrations from $6 \mathrm{w} / \mathrm{w} \%$, to $12 \mathrm{w} / \mathrm{w} \%$.

The initial set of experiments carried out used addition times of 60, 90 and 105 seconds (sample 58a, $58 \mathrm{~b}, 58 \mathrm{c}$ respectively), and utilised the CAB 381.01 polymer at $25 \mathrm{mg} / \mathrm{mL}$, PVA concentration of $3 \mathrm{w} / \mathrm{w} \%$ and $\mathrm{CaCl}_{2}$ concentration of $20 \mathrm{w} / \mathrm{w} \%$ for all the samples. The particles were purified in the usual fashion by three rounds of centrifugation and washing with deionised water and finally were freeze dried to remove water. SEM images of the samples showed them to be very similar to previously made samples with no significant changes in morphology. The DLS data (Figure 57) corroborates the SEM data as the particles sizes are relatively unchanged.
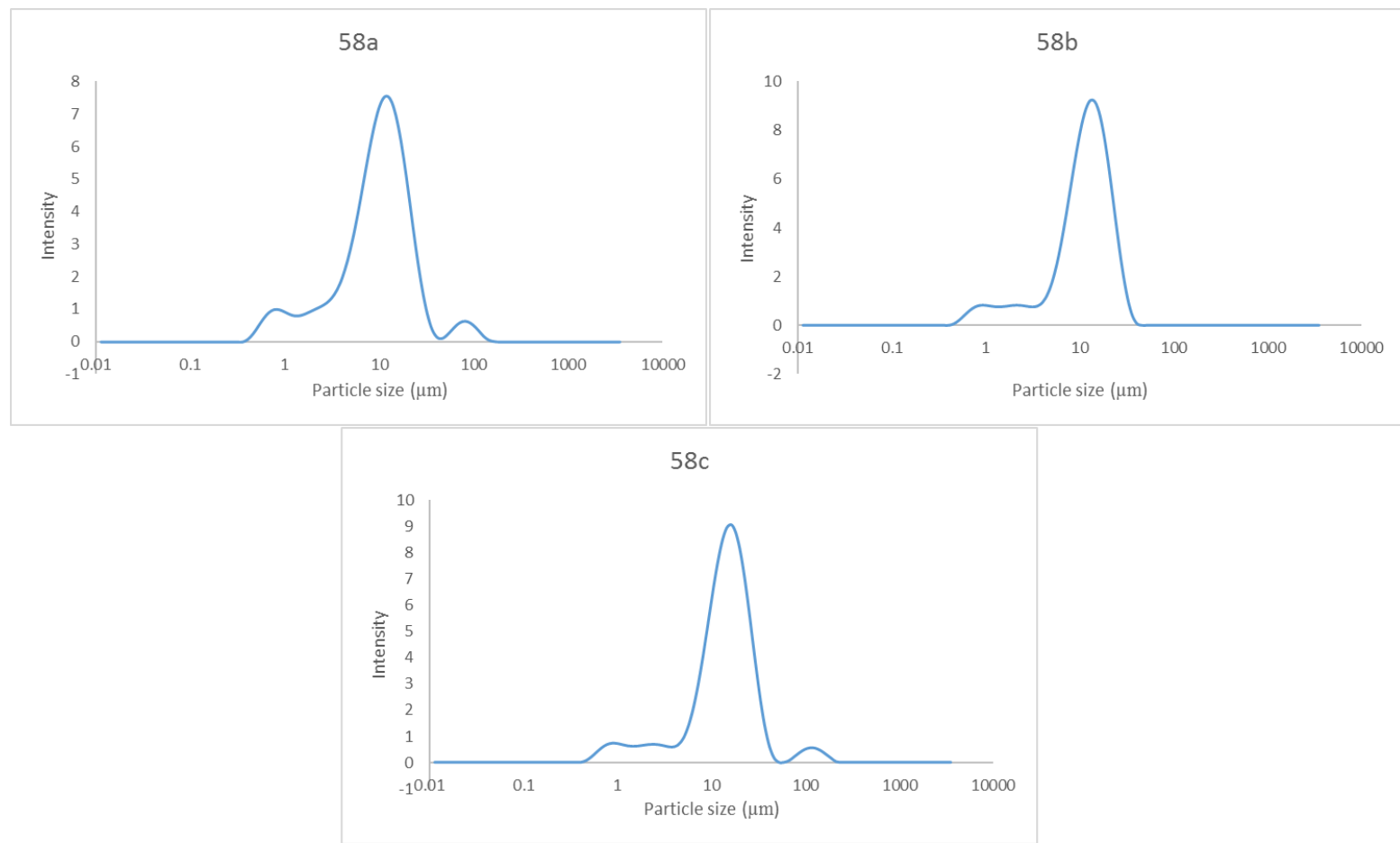

Figure 57: DLS data, varied addition times; 60s (top left), 90s (top right), 105s (bottom) 
The graphs above show that the size distribution has narrowed somewhat compared to previously prepared samples. The peak particle size is $12.7 \mu \mathrm{m}$, which is very close to the peak size of previous samples of around $13 \mu \mathrm{m}$. This shows that the only change to the particle size distribution has been the narrowing of the peak distribution (dispersity) but not the average particle size. This is exemplified by the D90 data (Table 8); sample 58a had a D90 of $24.4 \mu \mathrm{m}$, whereas sample $40 \mathrm{~d}$ had a D90 of $44.9 \mu \mathrm{m}$. This shows that sample 58a has far more of the bulk particles at smaller sizes to that of $40 \mathrm{~d}$. Although there is evidence for a bimodal distribution being generated in samples $58 \mathrm{a}$ and $58 \mathrm{c}$, both of which have higher D90s of $24.4 \mu \mathrm{m}$ and $30.4 \mu \mathrm{m}$ respectively. Thus, to achieve an even smaller average particle size, it was hypothesised that an increase in surfactant concentration would also be required.

Because the addition time of 60 seconds gave the best results, further experiments were carried out using this time in combination with increasing the surfactant concentration to $6 \mathrm{w} / \mathrm{w} \%, 9 \mathrm{w} / \mathrm{w} \%$ and $12 \mathrm{w} / \mathrm{w} \%$. The concentration of the cellulose polymer and calcium chloride was kept the same as the previous set of experiments. The SEM images taken of these samples directly after they were made showed there was significant coalescence occurring. The coalescence of these samples was later found to be due to insufficient purification, as the increased PVA concentration meant more centrifugation steps were required to achieve the same purity as previous samples. This was further demonstrated by the high yields attained from these samples of $75.4 \%, 71.2 \%$ and $93.9 \%$, which correspond to the samples using $6 w / w \%, 9 w / w \%$ and $12 w / w \%$ of PVA respectively. The high yields suggested that excess PVA was not being removed during the purification. To further this argument, the highest yield was attained from the sample with the highest PVA concentration. The excess PVA was removed from the samples using an extra round of centrifugation washing and freeze drying. After this second purification the particle size distribution was recorded (Figure 58), however no SEM was taken of these samples. 


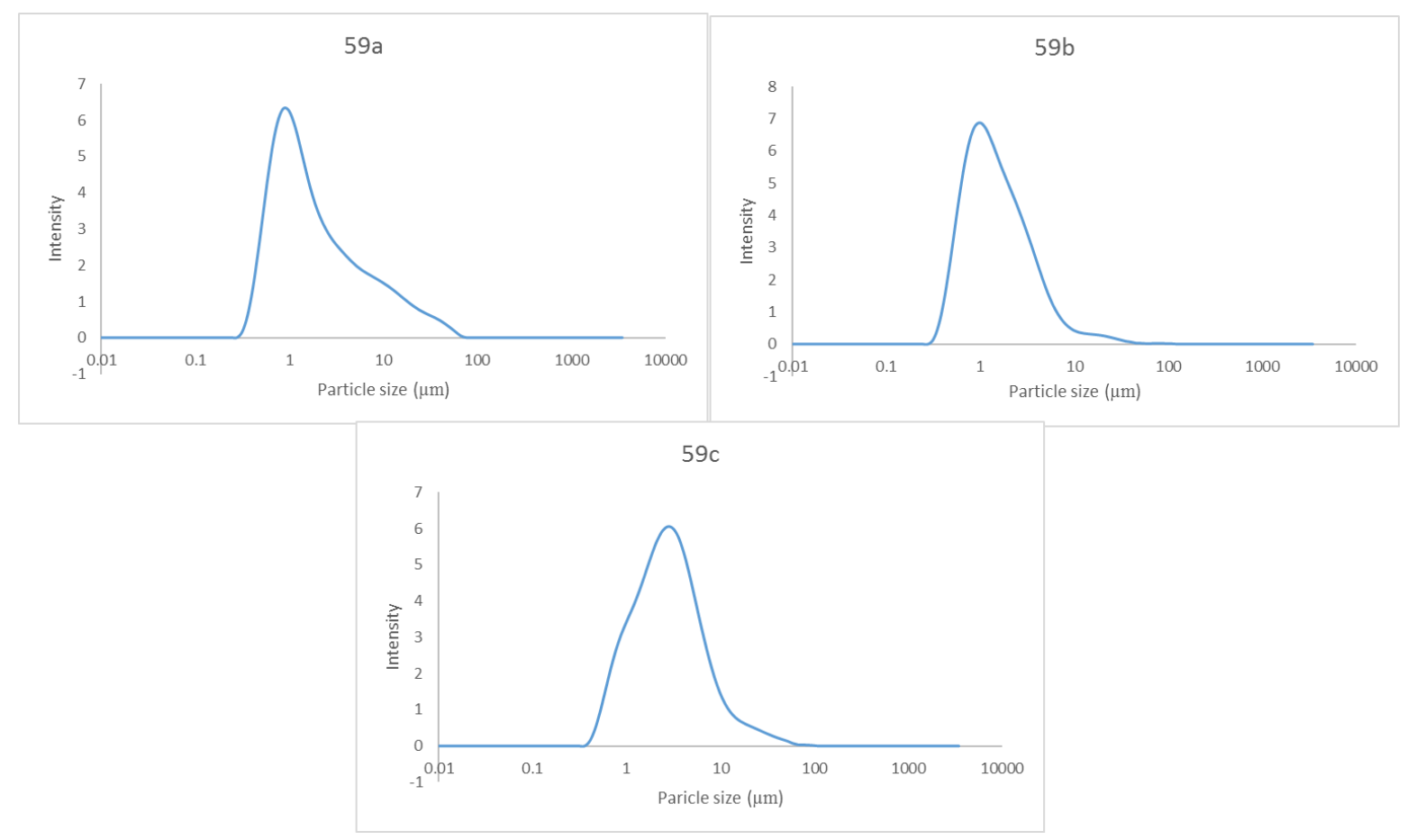

Figure 58: DLS data, varied PVA levels, $6 w / w \%$ (top left), $9 w / w \%$ (top right) and $12 w / w \%$ (bottom)

As can be observed in the above graphs, the particle size distribution has been reduced significantly, particularly in the first two samples where the peak maxima approaches $1 \mu \mathrm{m}$. The most monodisperse of the three concentrations tested was sample $59 \mathrm{~b}(9 \mathrm{w} / \mathrm{w} \%$ PVA). This decreased dispersity is apparent in the D90 values between the three samples; 59b has a D90 of $4.8 \mu \mathrm{m}$ while $59 \mathrm{a}$ is $12.1 \mu \mathrm{m}$. This indicated 59b has very few particles of the bulk sample above this value and, as such, has the narrowest distribution of the three. Also, the D10 and D50 values are similar between the three samples, with 59c having the highest of these values at 0.95 and $2.815 \mu \mathrm{m}$, respectively.

The success of this set of experiments led to further experiments being carried out using the addition time of 90 s with the PVA concentrations of $6 w / w \%$ and $9 w / w \%$. In addition, an assessment of salt $\left(\mathrm{CaCl}_{2}\right)$ concentration effects was completed as this had not been reported in the literature. Salt concentration was assessed at $30 \mathrm{w} / \mathrm{w} \%$ and $40 \mathrm{w} / \mathrm{w} \%$ (compared to $20 \% \mathrm{w} / \mathrm{w}$ in literature reports). The SEM images taken of samples (64a, 64b and 64c) showed the majority of particles had significantly decreased in size and also had a much smoother, more spherical shape (Figure 59). 

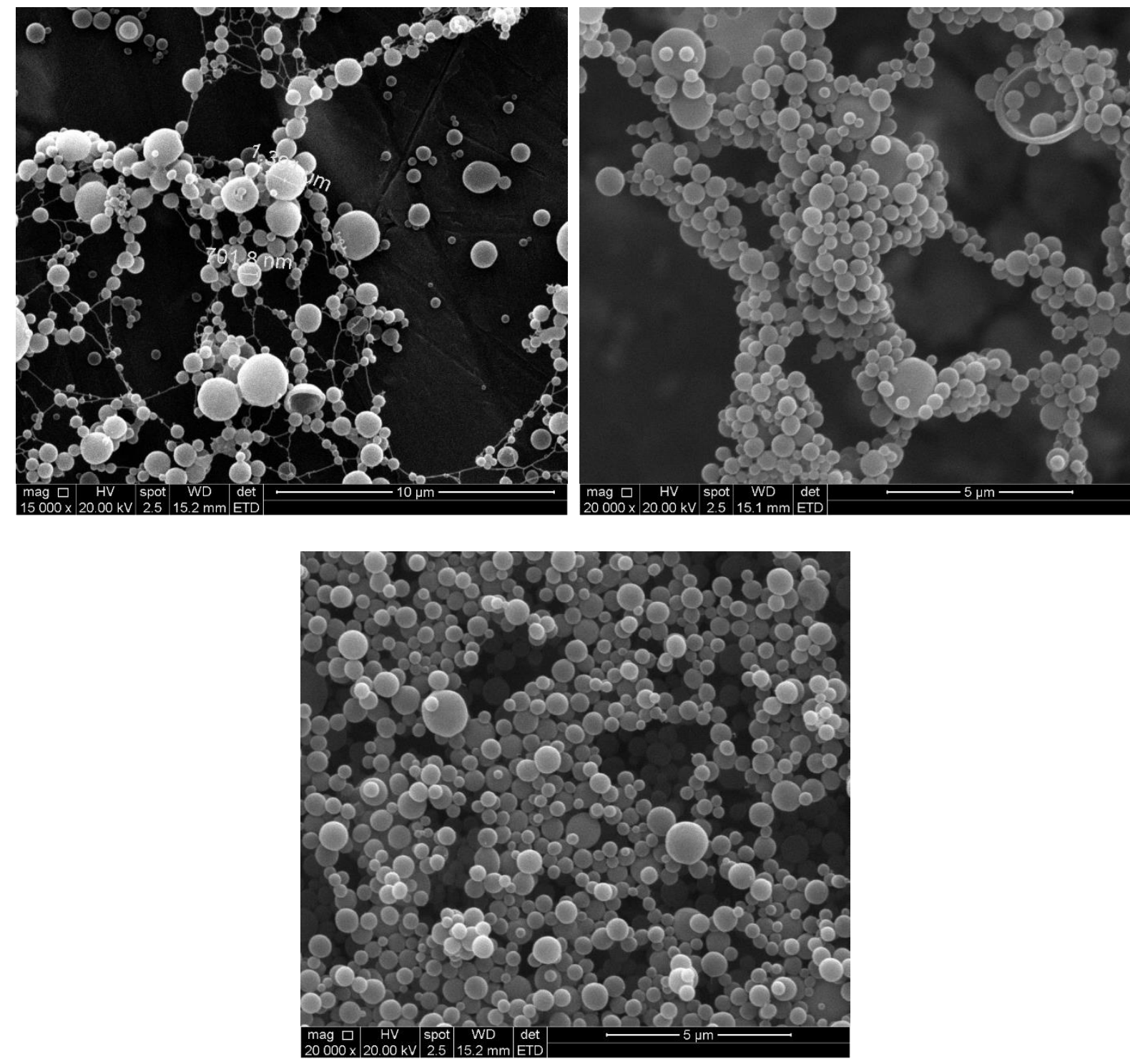

Figure 59: SEM images of samples; 6w/w\% PVA, 30w/w\% salt (top left), 9w/w\% PVA, 30w/w\% salt (top right) and $6 \mathrm{w} / \mathrm{w} \%$ PVA, 40w/w\% (bottom)

The SEM images above show the particles generated with varying salt and PVA concentrations. It was observed that there was a large population of particles below the $1 \mu \mathrm{m}$ size. However, there were some particles that were much larger in the $50 \mu \mathrm{m}$ range, with very little of the sample having sizes in between these two sizes. Each sample consistently showed this type of distribution, with some artefacts present. The DLS of these samples reflect the size distribution observed by SEM, with all the samples showing the presence of a bimodal distribution occurring (Figure 60). 


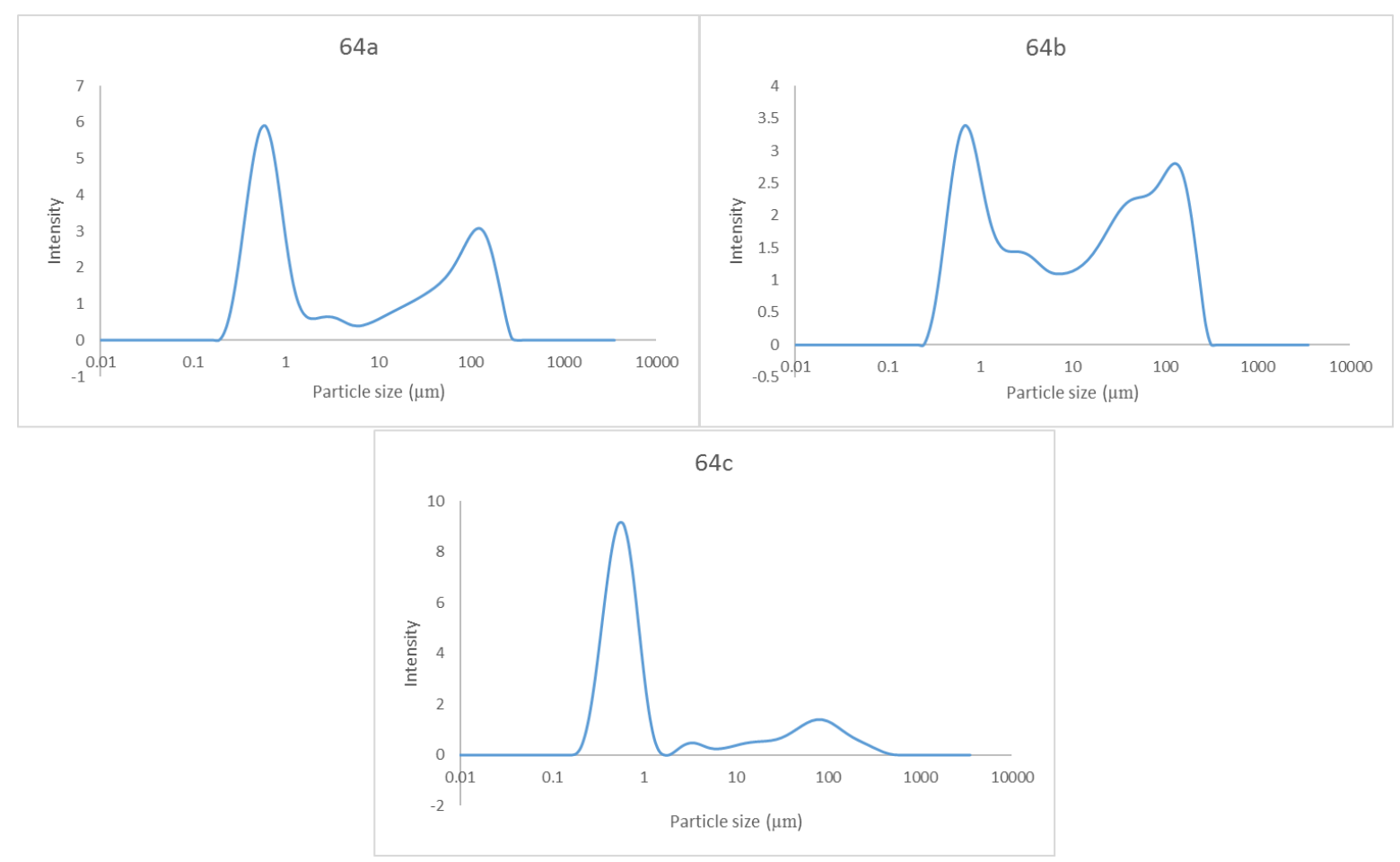

Figure 60: DLS data, salt and PVA content changes; 6w/w\% PVA, 30w/w\% salt (top left), 9w/w\% PVA, $30 \mathrm{w} / \mathrm{w} \%$ salt (top right) and 6w/w\% PVA, 40w/w\% (bottom)

The major peak for each sample was narrow, approximately centred on $600 \mathrm{~nm}$ for all three samples. A secondary distribution was present for particles of $\sim 100 \mu \mathrm{m}$, being most prominent in sample $64 \mathrm{~b}$ and least prominent in sample 64c; thus, higher PVA concentrations and higher salt concentrations could both be a contributor to the bimodal distribution.

The D90 values are affected to a great extent by this secondary population; sample 64b has a D90 of $130 \mu \mathrm{m}$, which reduces to $8.7 \mu \mathrm{m}$ in sample 59c. However, when comparing both the D10 and D50 values to the previous samples they are much lower, particularly for sample 64c. The lower D10 values of 0.37 to $0.63 \mu \mathrm{m}$ for the samples $64 a-c$ compared to samples 59a-c (Figure 58 ), show these samples have a higher population of particles below $400 \mathrm{~nm}$. This further demonstrates the change in the size distribution for these samples compared to the previous samples. It was concluded that the PVA concentration of $9 \mathrm{w} / \mathrm{w} \%$ was too high for this system and was causing coalescence to occur too frequently. In addition, purification of the particles was becoming much more difficult.

The bimodal distribution observed was hypothesised to be due to the consequence of both catastrophic and transitional phase inversion occurring at the same time. ${ }^{56}$ This phenomena was observed in our study for all addition times between 20 to 40 seconds (Figure 61). 


\section{This content is unavailable.}

\section{Please consult the print}

\section{version for access.}

Figure 61: Particle size distributions of the different addition times of aqueous phase in a phase inversion experiment $^{57}$

The difference in the addition time for which the bimodal distribution was observed is likely due to the different formulation being tested in the literature compared to the one used in this project. Also shown in the above figure are the optical micrographs reported of samples using addition times of 1200s (b) and 5s (c). These images clearly show the difference in particle sizes at the extreme ends of the addition times tested and this result is somewhat reflected in the samples 64a-c, where the primary distribution is narrow and the secondary distribution is broader, as shown above.

Table 8: D-values for particle size optimisation of phase inversion method

\begin{tabular}{lllllll}
\hline $\begin{array}{l}\text { Sample } \\
\text { number }\end{array}$ & $\begin{array}{l}\text { PVA } \\
\text { concentration } \\
\text { (w/w\%) }\end{array}$ & $\begin{array}{l}\text { Addition } \\
\text { time } \\
\text { (seconds) }\end{array}$ & $\begin{array}{l}\text { Salt } \\
\text { concentration } \\
(\mathbf{w} / \mathbf{w})\end{array}$ & D10 $(\boldsymbol{\mu m})$ & D50 $(\boldsymbol{\mu m})$ & D90 $(\boldsymbol{\mu m})$ \\
\hline $\mathbf{5 8 a}$ & 3 & 60 & 20 & 2.24 & 10.87 & 24.43 \\
\hline $\mathbf{5 8 b}$ & 3 & 90 & 20 & 3.11 & 12.59 & 23.53 \\
\hline $\mathbf{5 8 c}$ & 3 & 105 & 20 & 4.44 & 15.42 & 30.34 \\
\hline $\mathbf{5 9 a}$ & 6 & 60 & 20 & 0.63 & 1.55 & 12.11 \\
\hline $\mathbf{5 9 b}$ & 9 & 60 & 20 & 0.64 & 1.43 & 4.77 \\
\hline $\mathbf{5 9 c}$ & 12 & 60 & 20 & 0.95 & 2.81 & 8.71 \\
\hline $\mathbf{6 4 a}$ & 6 & 90 & 30 & 0.43 & 1.54 & 131.87 \\
\hline $\mathbf{6 4 b}$ & 9 & 90 & 30 & 0.63 & 10.29 & 130.28 \\
\hline $\mathbf{6 4 c}$ & 6 & 90 & 40 & 0.37 & 0.70 & 78.77 \\
\hline
\end{tabular}


The particle size optimisation of the phase inversion process was driven by discoveries made in the literature about the nature of phase inversion ${ }^{56}$ and how to manipulate the mechanism that predominates. ${ }^{57}$ It was found that the addition time of the aqueous is critical and a time of 90 seconds gave the most ideal particle size distributions. Also, the concentration of PVA and the calcium chloride was found to be a critical component in generating the ideal particle sizes. These were found to be $6 w / w \%$ of PVA and $40 w / w \%$ of calcium chloride in the organic and aqueous phases respectively. The next step was to use this optimised formulation to encapsulate three target fungicides.

\subsubsection{Model compound incorporation}

The next stage in developing the phase inversion method was to incorporate a model compound to test the robustness of the process and its ability to carry and deliver an active compound. A second goal was to develop a method suitable for the detection of the active ingredient in the particles produced. Bipyridine was used as the model compound because its structural features were not dissimilar to the insecticides that would be ultimately utilised, and it has a chromophore for ready detection.

The method was unchanged from that described in section 3.7.1, except that bipyridine was dissolved into the organic phase before the particles were formed. Concentrations of $0.5 \%, 1 \%$ and $1.5 \%$ (sample $47 \mathrm{a}, 47 \mathrm{~b}$ and $47 \mathrm{c}$ respectively), of bipyridine were used to assess the compound's effect on the particles (Figure 62). These images show that the morphology of the particles remained relatively unchanged with bipyridine incorporation at all concentrations. DLS data showed no difference in the particle size distribution compared with that of the blank samples (Table 9).

When comparing the D-values of previously prepared samples to those formulated with bipyridine they are very similar, except for the D90 values. On average the D90 values obtained for the blank particles were higher than that of the particles prepared with bipyridine; $41 \mu \mathrm{m}$ and $23 \mu \mathrm{m}$ respectively. This would indicate that the previous samples had higher populations of larger particles. This may be due the different $C A B$ polymers used in the previous experiments, leading to more phase inversion of the catastrophic nature of emulsion collapse resulting from higher viscosity organic phases. The next step was to use a spectroscopic method to quantify the bipyridine in the particles and the supernatant to see if it was being incorporated into the particles. The spectroscopic technique utilised for this was infrared spectroscopy. 

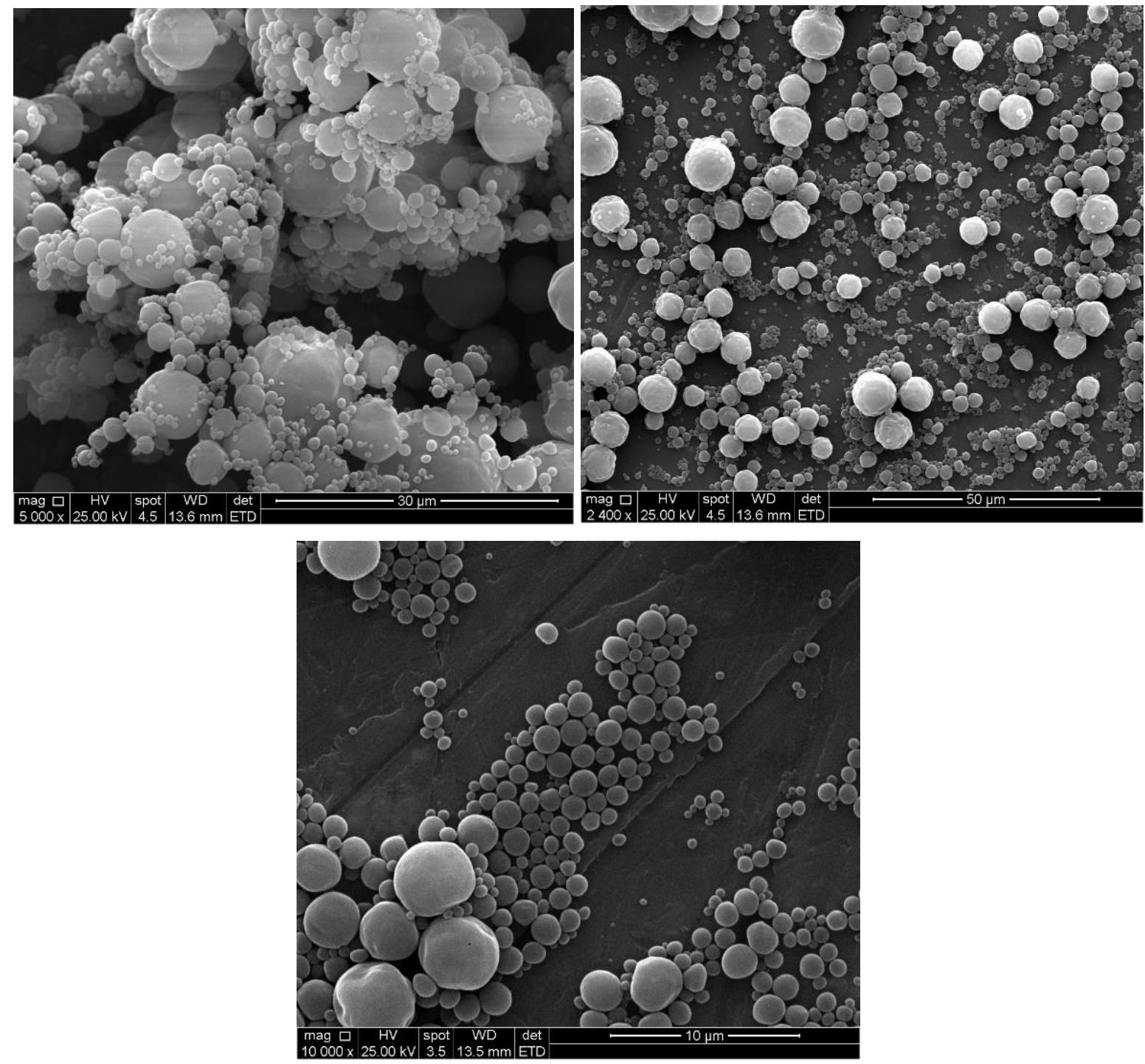

Figure 62: SEM images for samples 47a (top left), 47b (top right) and 47c (bottom)

The first step in using IR to analyse the particles containing bipyridine was to find the diagnostic peaks that would be used to identify the polymer and bipyridine (Figure 63). The spectra of these are shown below and the diagnostic peaks are highlighted. Each diagnostic peak was selected due to its isolation from other peaks as the spectra of the particles will contain both these compounds. For the $C A B$, the carbonyl peak at $1740 \mathrm{~cm}^{-1}$ was selected; given that bipyridine has no carbonyl functional groups this peak was isolated from any other peaks. For bipyridine the diagnostic peak selected was the strong peak at $753 \mathrm{~cm}^{-1}$, again due to its isolation from other noise and its intensity, given it resides in the fingerprint region of the spectra (tentatively attributed to the out of plane $\mathrm{C}-\mathrm{H}$ bend). The expectation was that with increasing concentration of bipyridine the diagnostic peak would increase in size relative to the peaks associated with the CAB. 

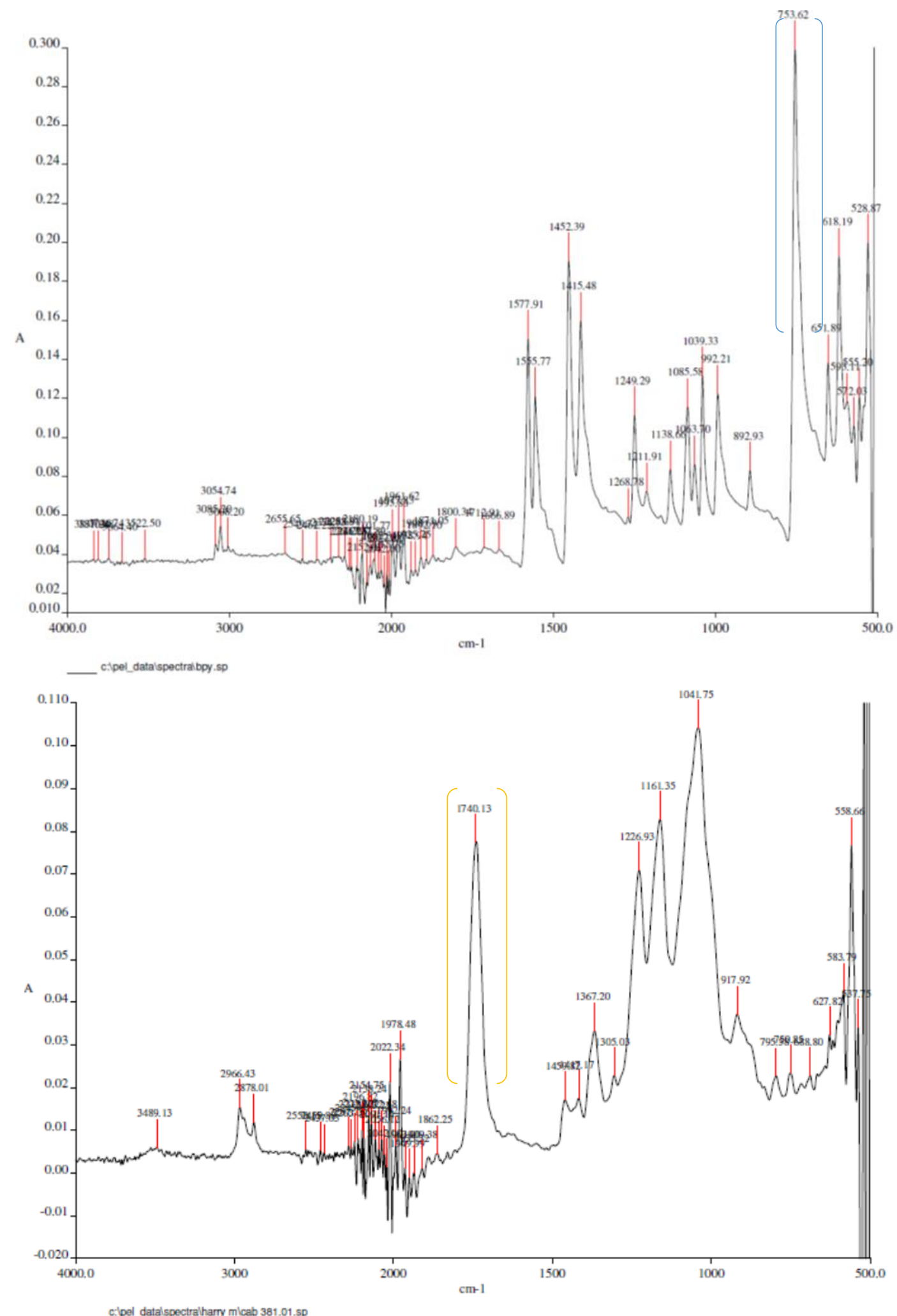

Figure 63: IR spectra of bipyridine (top) and CAB 381.01 (bottom)

In the next set of experiments, the concentration of bipyridine was increased to $5 \%, 8.4 \%$ and $12.3 \%$. The SEM images of these samples showed some changes to the previous three samples in morphology and in particle size distribution. It was observed in the SEM that some of the particles at these 
concentrations had collapsed or split in half. This phenomenon has been observed in many other samples generated by phase inversion and is likely an artefact of drying the samples before SEM, however these samples did show much higher occurrence of this than the blank samples. The SEM image shown below in Figure 64 shows this phenomenon.

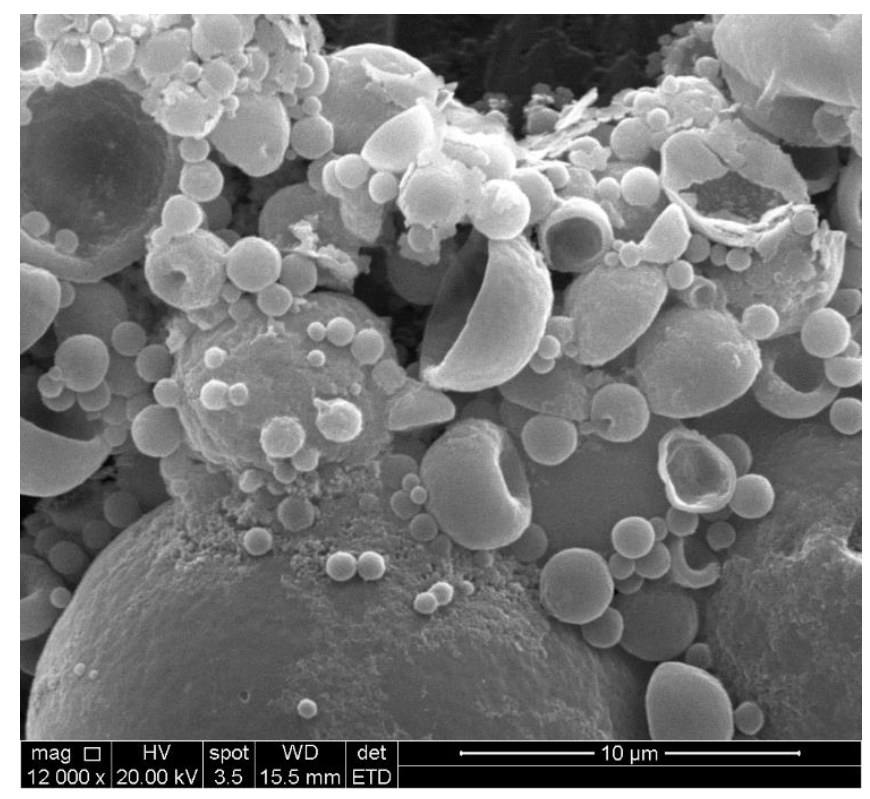

Figure 64: SEM image of sample 50b, showing particle degradation

In the above figure it can also be observed that the large particle towards the bottom of the image is rougher than other samples generated previously. These higher concentration bipyridine samples all share this morphology on the larger particles. This could potentially be caused by the bipyridine dropping out of solution at the interface before the $C A B$ during particle formation, thus leading to the rough layer observed on the larger particles. This rough layer is also more prominent at higher concentrations of bipyridine, which leads to the conclusion that the loading capacity of the particles has been reached at these higher concentrations. But yields obtained for each of the samples (Table 9), decreases with each successive experiment. This suggests that the majority of the bipyridine was not being incorporated into the particles in the samples with high bipyridine concentrations. Excess bipyridine may be forming this rough layer on the exterior of the particles. This may have also led to the increase in size of the diagnostic peaks for bipyridine in the IR spectra collected for these sample (shown in the appendix).

This model compound testing demonstrated that the formulation was able to successfully encapsulate an active compound, along with demonstrating that this active compound was incorporated into the particles using IR as a method of detection. However, given the difficulty in detecting bipyridine quantitatively with the IR method, this testing was ended here in favour of incorporating the target fungicides. 
Table 9: Data for model compound formulations

\begin{tabular}{llllll}
\hline $\begin{array}{l}\text { Sample } \\
\text { number }\end{array}$ & $\begin{array}{l}\text { Bipyridine concentration } \\
(\mathbf{m g} / \mathbf{m L})\end{array}$ & Yield (\%) & D10 $(\mu \mathrm{m})$ & D50 $(\mu \mathrm{m})$ & D90 $(\mu \mathrm{m})$ \\
\hline $\mathbf{4 7 a}$ & 5 & $44.74 \%$ & 1.97 & 8.66 & 20.25 \\
\hline $\mathbf{4 7 b}$ & 10 & $47.05 \%$ & 2.35 & 9.71 & 19.30 \\
\hline $\mathbf{4 7 c}$ & 15 & $40.67 \%$ & 2.02 & 7.96 & 17.45 \\
\hline $\mathbf{5 0 a}$ & 50 & $37.6 \%$ & 2.69 & 7.68 & 17.31 \\
\hline $\mathbf{5 0 b}$ & 84 & $35.46 \%$ & 2.73 & 8.53 & 23.42 \\
\hline $\mathbf{5 0 c}$ & 123 & $29.1 \%$ & 2.88 & 6.52 & 13.30 \\
\hline
\end{tabular}

\subsubsection{Fungicide incorporation}

The compounds targeted for incorporation into the cellulosic particles were three industry-standard fungicides; Triadimefon, Cyproconazole and Tebuconazole (Figure 65).<smiles>CC(C)(C)C(=O)C(Oc1ccc(Cl)cc1)n1cncn1</smiles>

Triadimefon

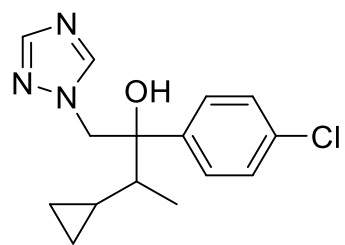

Cyproconazole<smiles>CC(C)(C)C(O)(CCc1ccc(Cl)cc1)Cn1cncn1</smiles>

Tebuconazole

Figure 65: Structures of the three fungicides used in this project

These fungicides all share the triazole moiety, which is a common functional group in fungicides. The solubility of these fungicides is ideal for the project application; all three have water solubility values between $32 \mathrm{mg} / \mathrm{L}$ and $64 \mathrm{mg} / \mathrm{L}$, and are soluble in common organic solvents such as dichloromethane, toluene, acetone and ethanol. They are also well researched and relatively safe for humans. ${ }^{80}$

Two experiments were initiated using 1:1 and 1:1.5 weight ratios of polymer to fungicide. The optimised procedure described in section 3.7.2 was used. The different mass-to-polymer ratios were selected to assess the loading capacity of the particles and if morphologies would change with a greater fungicide to polymer ratio. This was particularly relevant considering the effects observed when pigment or higher model-compounds were used (section 2.4.4). The particles that were generated appeared under SEM to be unchanged in morphology to the blank samples using the optimised method (Figure 66). 

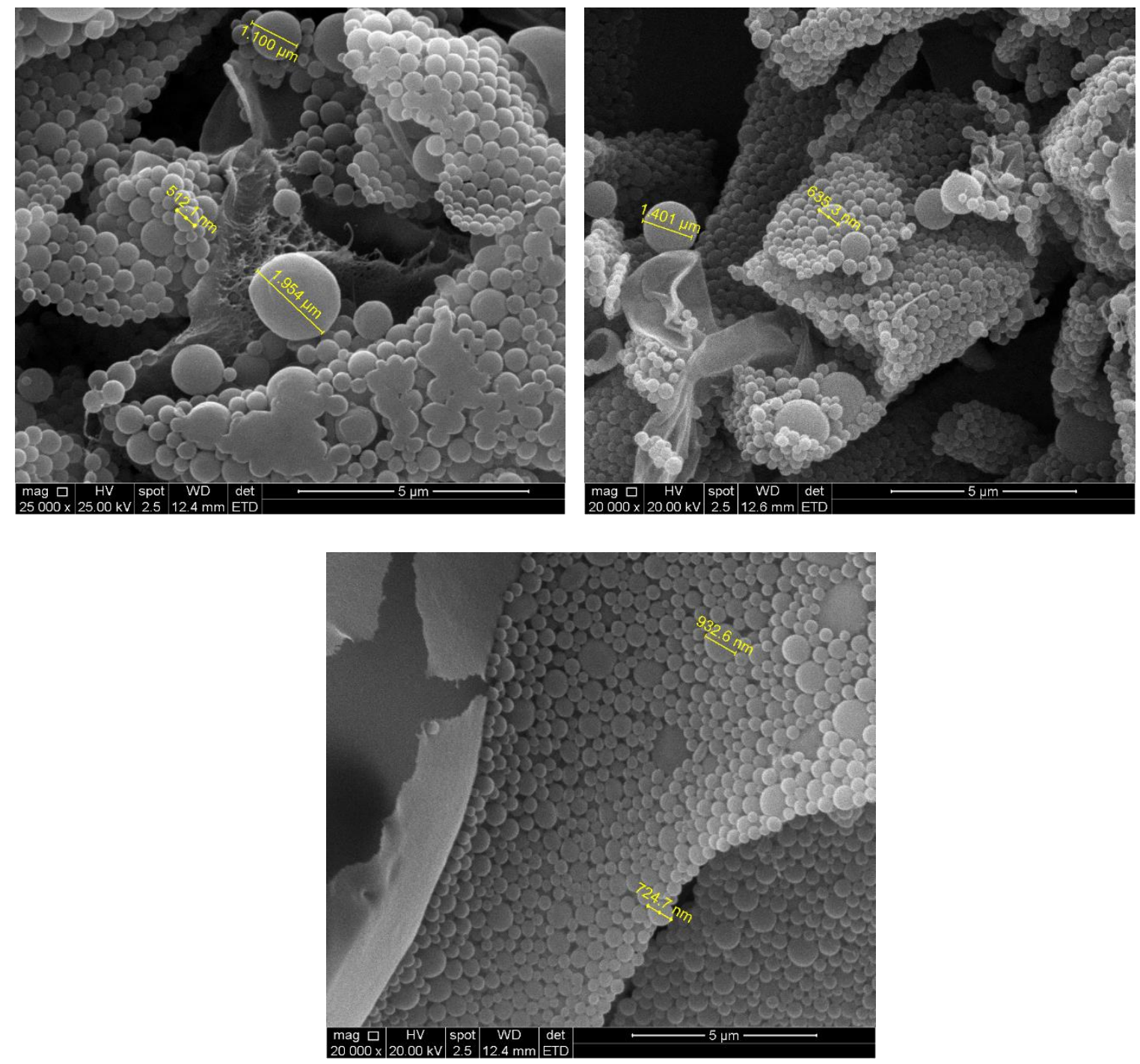

Figure 66: SEM images of samples 98a (top left), 98d (top right) and 98e (bottom)

The images show a consistent particle size has been obtained, between $700 \mathrm{~nm}$ to $1200 \mathrm{~nm}$ for the majority of the particles in each sample. There were anomalies observed in each sample, such as large particles and burst particles, however this should not impact the outcome of incorporating these particles into wood as only the smaller particles will penetrate the pores of the wood. The abnormal particles were observed in small numbers so they only represent the minority of the population of particles.

The DLS of these samples show the bimodal distribution that was found in the previous experiments. Some of the samples have a very small secondary distribution of larger particles compared to others, indicating a possible inconsistency in the production of these samples (Figure 67). 


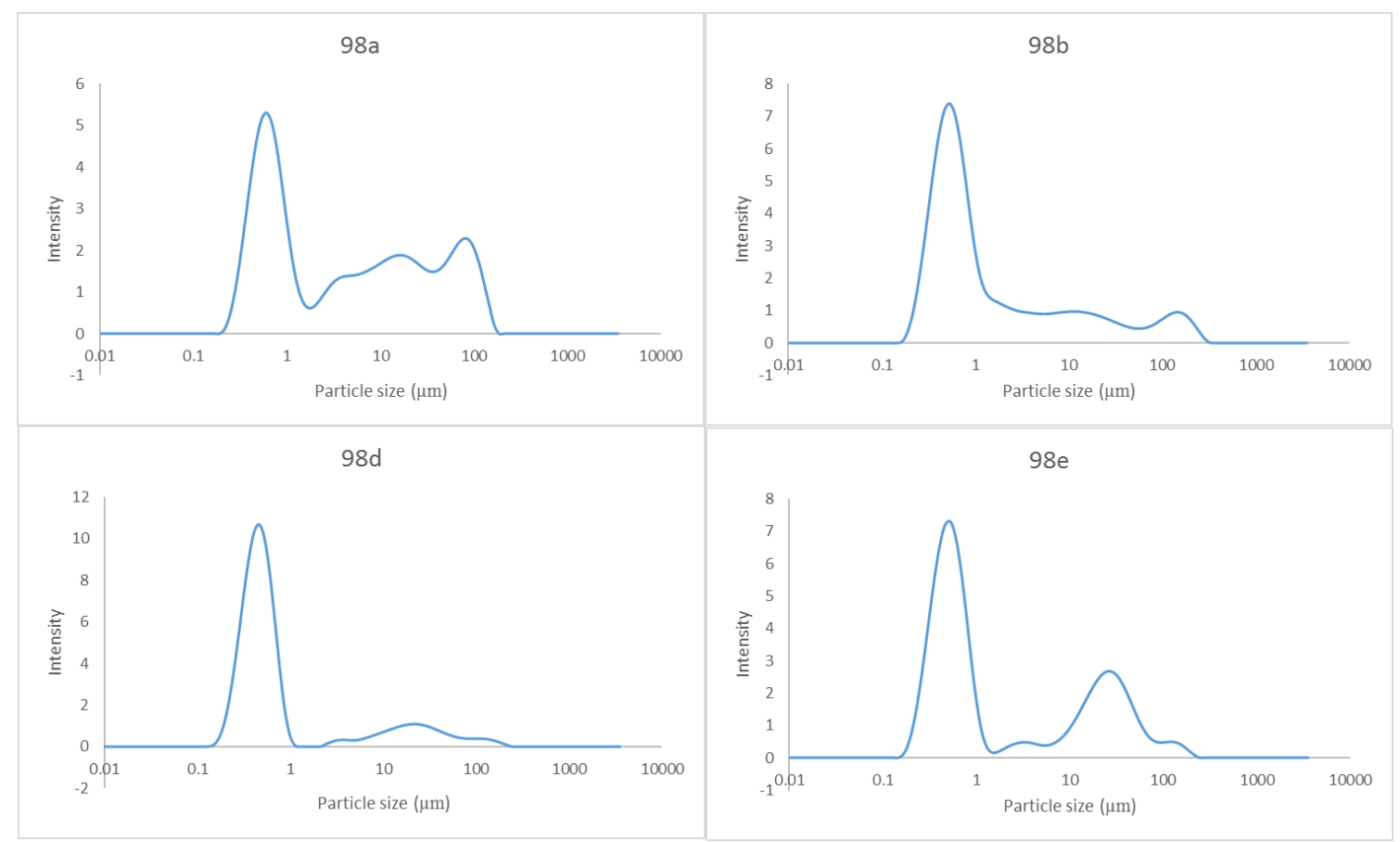

Figure 67: DLS for samples containing fungicide; 98a (Triadimefon), 98e (Tebuconazole) (1:1 mass ratio) and 98b (Triadimefon), 98d (Cyproconazole) (1:1.5 mass ratio)

The DLS graphs show the major distribution to have a peak value of $400 \mathrm{~nm}$ for each sample with a very similar width (dispersity) for this primary distribution. Samples 98b and 98d have the smallest secondary distribution. This is evidenced by the D90 values of 35 and $22 \mu \mathrm{m}$ respectively compared to 77 and $40 \mu \mathrm{m}$ for samples 98a and 98e respectively. Both the D10 and D50 values are relatively close between the four samples, with the only exception being that of the D50 for sample $98 \mathrm{a}$ of $3.5 \mu \mathrm{m}$ (compared to 0.76 to $0.5 \mu \mathrm{m}$ for the other three samples). The second distribution is much broader and far smaller in population to the primary and is also somewhat variable between samples, with sample 98a having the broadest and largest secondary distribution. Again, this is further evidence for the hypothesis that both transitional and catastrophic phase inversion are occurring together, although transitional is clearly occurring more than catastrophic in this set of experiments compared to the previous blank optimised experiments.

To confirm the inclusion of the fungicides in the samples, ${ }^{1} \mathrm{H}$ NMR was completed on samples $98 \mathrm{e}, 98 \mathrm{~d}$ and 98a, which each had one of the three fungicides incorporated (Figure 68). Each spectrum was collected by dissolving the particles (10mg approximately) in deuterated chloroform. Reference spectra were also collected for the CAB 551.01 and the three fungicides tested here (see appendix). 


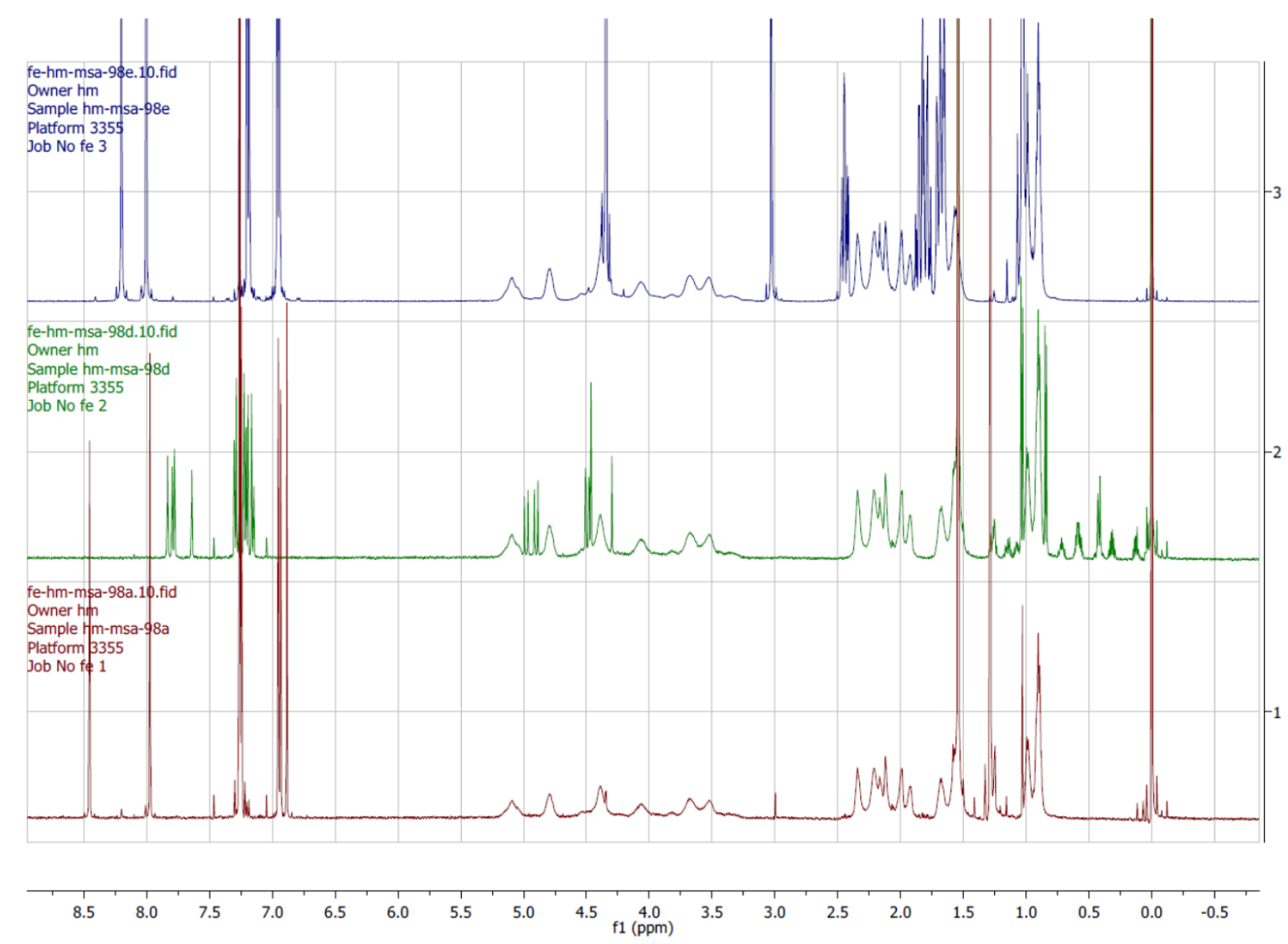

Figure 68: ${ }^{1} \mathrm{H}$ NMR $\left(\mathrm{CDCl}_{3}\right)$ spectra of samples; $98 \mathrm{e}$ (top, Tebuconazole), 98d (middle, Cyproconazole) and 98a (bottom, Triadimefon)

The stacked spectra above show that both CAB 551.01 and the fungicides are present in each sample. The broad peaks between $5 \mathrm{ppm}$ and $3.5 \mathrm{ppm}$ are indicative of the protons associated with the anhydroglucose unit of the CAB 551.01 polymer, while broad peaks at 2.2ppm correspond to the cellulose-acetate protons. The remaining sharper peaks are consistent with the fungicide peaks. These spectra prove that the fungicides are being incorporated into the particles. IR spectra were also completed on these samples however they were not diagnostic for fungicide incorporation.

The final step was to produce these fungicide-incorporated samples in bulk quantities for their subsequent biocide testing by our industrial partners Lonza. The samples were produced using the 1:1 weight ratio of cellulose to fungicide, however they were produced at a $5 \mathrm{~g}$ scale. The yields of these bulk scale experiments proved to be fairly low; an average of 35\% was achieved. The low yields were likely because the Ultra-Turrax used in this project was at its volume limit when carrying out these large-scale experiments. This would potentially lead to the sheer being applied to a more local area in the reaction vessel, similar to effects described for high viscosity solutions. However, the particles still form in the sizes as shown with the small-scale experiments (98a-d), only less of the dissolved fungicide and polymer form into particles. To produce enough material for the biocide testing, the $5 \mathrm{~g}$ scaled-up process was completed twice. DLS (Figure 69) and SEM analysis was done on the samples to check that a consistent product had been generated. 


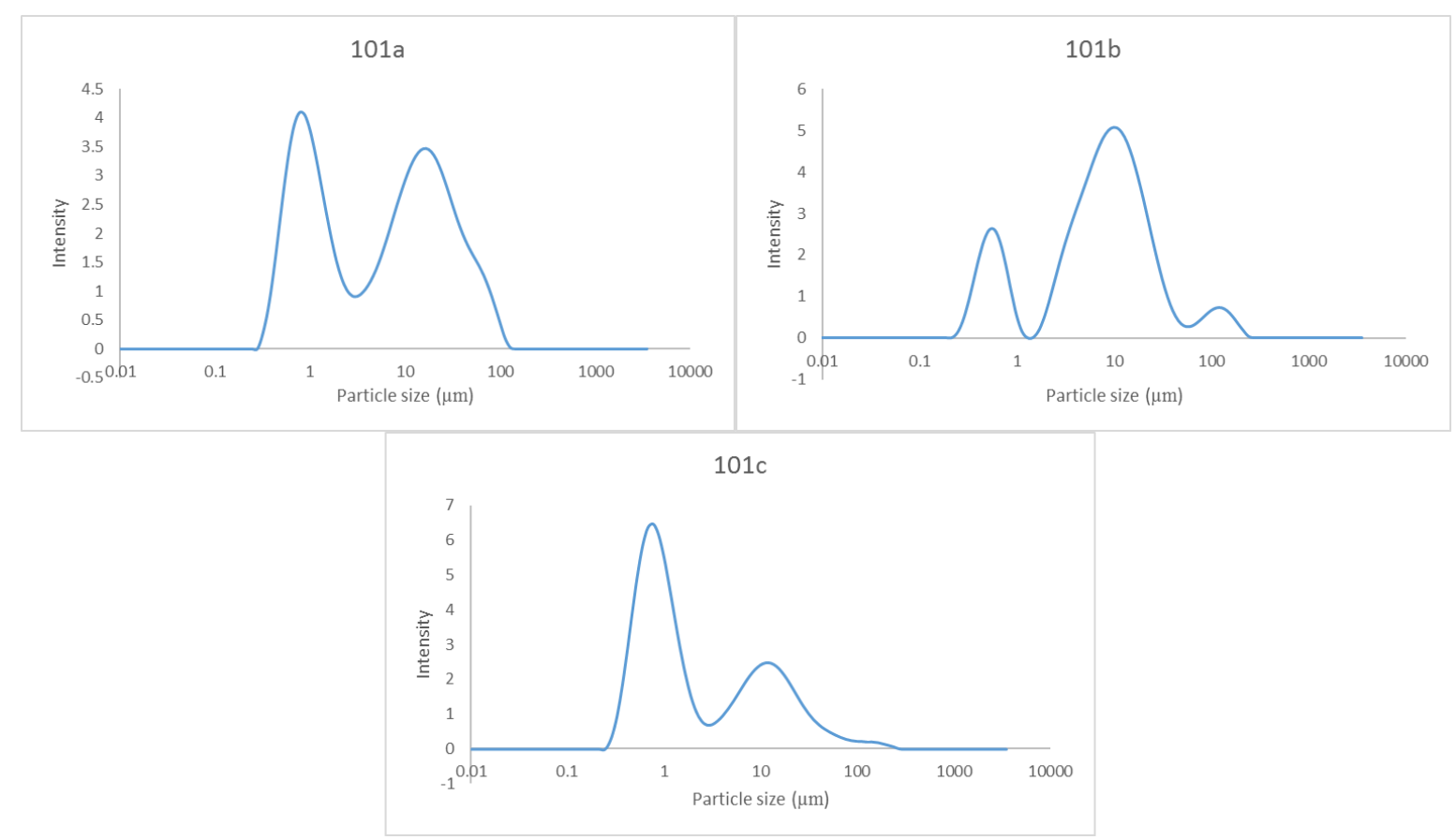

Figure 69: DLS data for scaled up samples containing each fungicide; 101a (top left, Triadimefon), 101b (top right, Cyproconazole), and 101c (bottom, Tebuconazole)

The DLS graphs of the bulk scale samples show a significant difference in particle size distribution to the smaller scale samples. The D50 values (Table 10) for the bulk samples shows this difference very clearly as they are significantly higher than that of the small-scale experiments. To compare, the D50 for the large-scale sample using Cyproconazole, $(101 \mathrm{~b})$ is $8.4 \mu \mathrm{m}$, whereas the D50 for the small-scale sample also using Cyproconazole (98b) is $0.76 \mu \mathrm{m}$. This difference in D50 values demonstrated that in the scale-up material a much greater proportion of larger particles had been generated.

The only sample of the bulk experiments that has a lower D50 was the sample using Tebuconazole (101c), which has a D50 of $1.3 \mu \mathrm{m}$. It is not known why sample 101c had a size distribution that was much closer to the small-scale samples, as all the large-scale samples were prepared in the same fashion. This may be simply due to the variation in the conditions under which the samples were made and/or whether the samples were left to stand for an extended period, which could affect agglomeration. The particle size distribution was attributed to aggregation of the particles while being analysed by the DLS. The SEM of the large-scale samples showed a very similar particle size distribution as what was observed with the small-scale samples. It could be concluded that more aggregation may have occurred (as detected by the Zetasizer) due to the extended period required to purify the larger batch, which may lead to more particles coming into contact. 

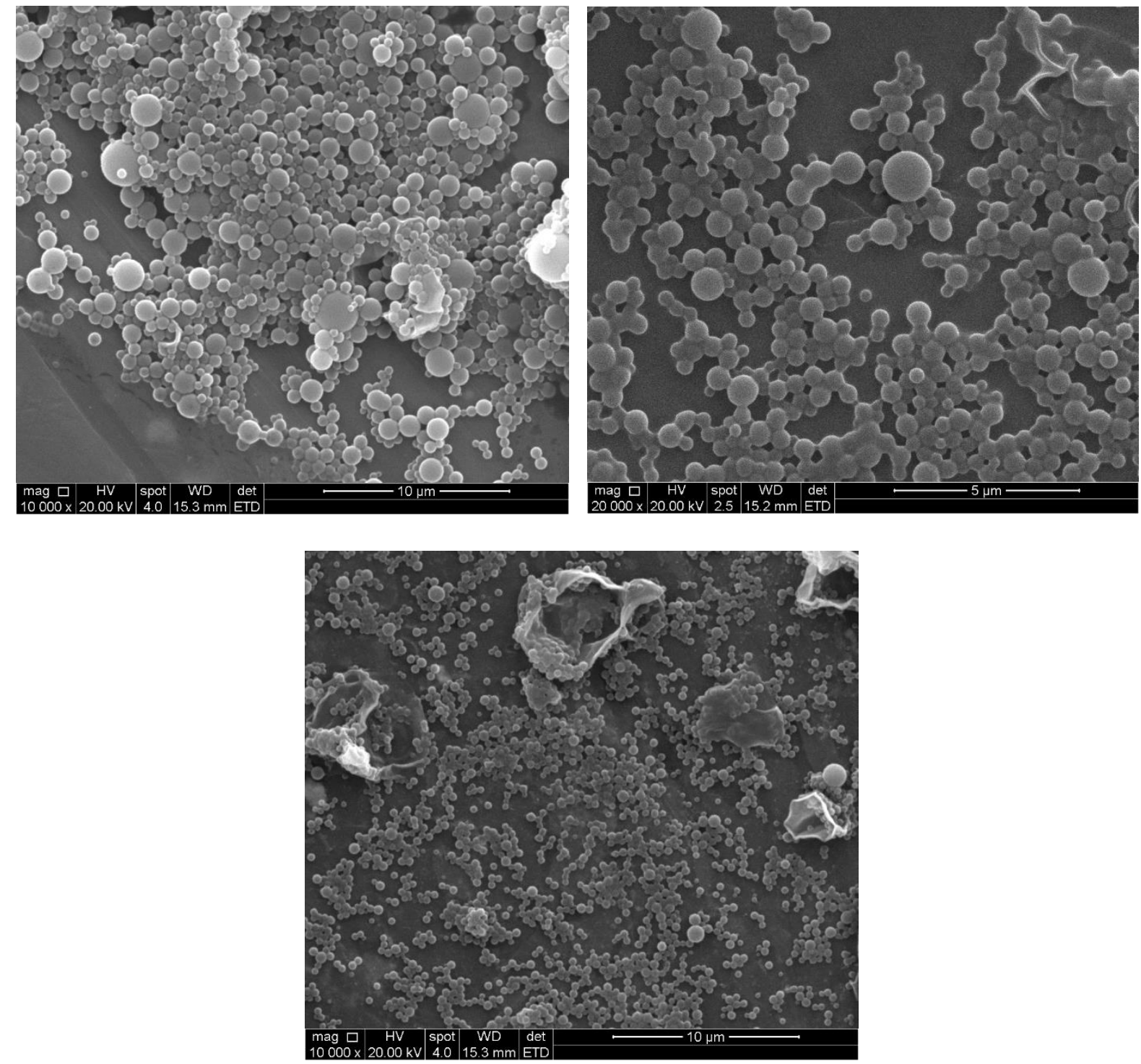

Figure 70: SEM images for samples; 101a (top left), 101b (top right) and 101c (bottom)

The SEM images demonstrate that samples produced in bulk scale were very similar to the smaller scale experiments in particle size and morphology (Figure 70). However, the DLS data showed conflicting data, with a much larger population of larger particles which is not reflected in the SEM images. As previously mentioned, aggregation of the particles could be occurring when they are analysed by the DLS. To see if this was true, a further set of DLS experiments were carried out, with the added step of sonicating the samples while dispersed in water for 2 hours before being analysed. Samples are normally sonicated before analysis as per the experimental procedure. However, the larger scale samples may experience greater aggregation than the smaller scale samples, thus requiring longer sonication times to break up aggregates. The long sonication time prior to DLS corroborates the hypothesis made previously (Figure 71). 

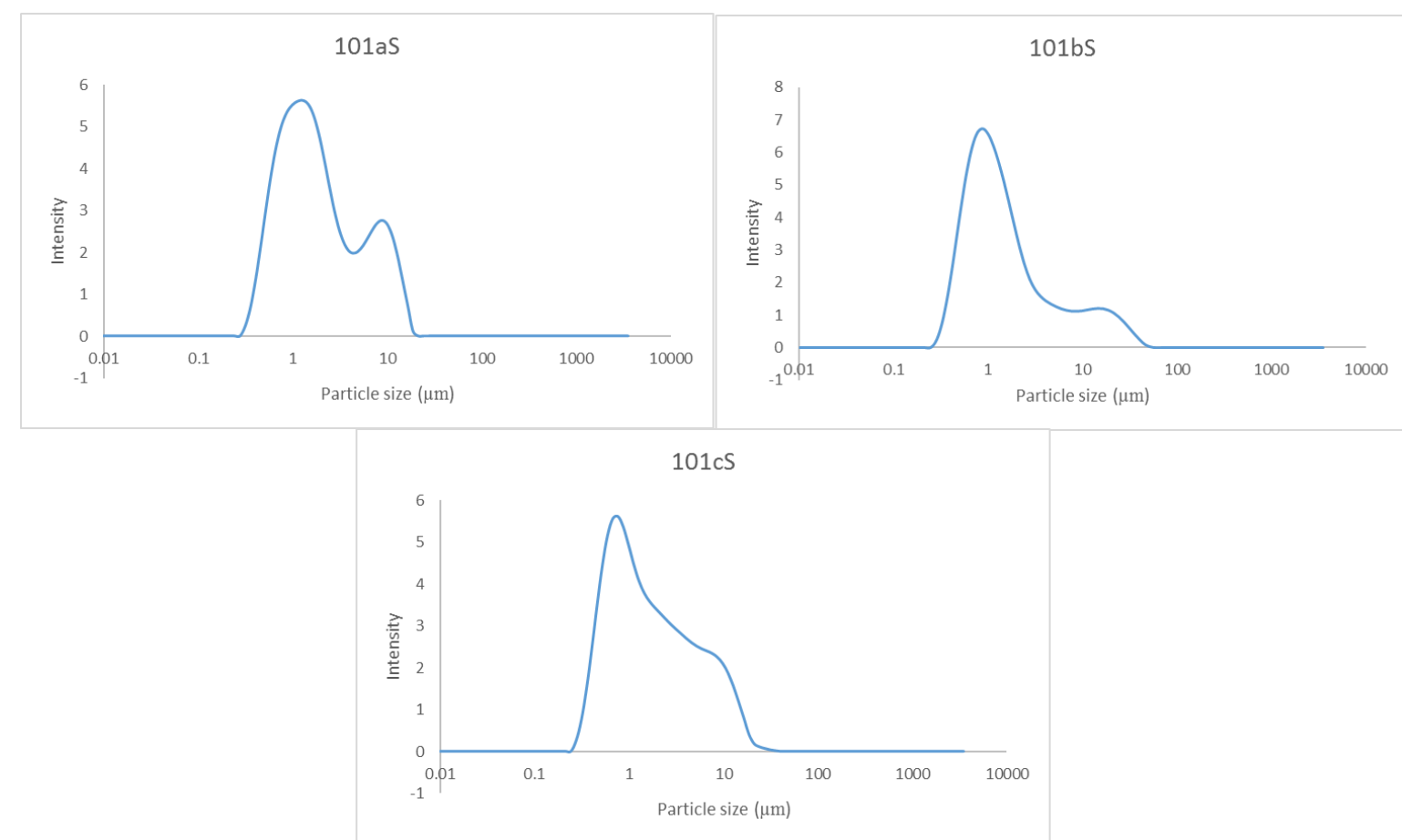

Figure 71: DLS data for scaled up samples sonicated for 2 hours prior to acquiring particle size data

The graphs above clearly show much better particle size distributions than those found previously. The D90 values of these sonicated samples compared to those from the non-sonicated samples clearly show a significant reduction in the secondary distribution size. The D90 for sample $101 \mathrm{bS}$ was $10.75 \mu \mathrm{m}$ compared to sample $101 \mathrm{~b}$, with a D90 of $29.9 \mu \mathrm{m}$. This shows a significant improvement in narrowing the particle size distribution, which is also evidenced by observing the difference in the graphs of these two samples. Also, the D10 and D50 values remain relatively unchanged, which further shows that high aggregation of the samples was occurring during these measurements. However, the distributions do differ slightly from that of the smaller scale experiments and this is likely due to the Ultra Turrax working on a much larger volume, resulting in the sheer becoming more localised. This may have led to more catastrophic phase inversion occurring. Care was taken not to exceed the capacity of the Ultra Turrax (which is $50 \mathrm{~mL}$ total volume) and the experiments were broken down into three separate particle forming procedures. 
Table 10: D-values for particle with fungicide incorporated into the formulation

\begin{tabular}{lllllll}
\hline $\begin{array}{l}\text { Sample } \\
\text { name }\end{array}$ & Fungicide & $\begin{array}{l}\text { Mass ratio } \\
(\mathbf{F : P})^{\mathbf{1}}\end{array}$ & $\mathbf{D 1 0}(\boldsymbol{\mu m})$ & D50 $(\boldsymbol{\mu m})$ & D90 $(\boldsymbol{\mu m})$ & Yield $(\%)$ \\
\hline $\mathbf{9 8 a}$ & Triadimefon & $1: 1$ & 0.46 & 3.55 & 77.81 & N/A \\
\hline $\mathbf{9 8 b}$ & Triadimefon & $1.5: 1$ & 0.35 & 0.76 & 35.57 & N/A \\
\hline $\mathbf{9 8 d}$ & Cyproconazole & $1.5: 1$ & 0.30 & 0.53 & 22.11 & N/A \\
\hline $\mathbf{9 8 e}$ & Tebuconazole & $1: 1$ & 0.34 & 0.75 & 40.62 & N/A \\
\hline $\mathbf{1 0 1 a}$ & Triadimefon & $1: 1$ & 0.66 & 7.18 & 41.88 & 36 \\
\hline $\mathbf{1 0 1 b}$ & Cyproconazole & $1: 1$ & 0.61 & 8.42 & 29.92 & 60.9 \\
\hline $\mathbf{1 0 1 c}$ & Tebuconazole & $1: 1$ & 0.53 & 1.31 & 22.80 & 30.3 \\
\hline $\mathbf{1 0 1 a \mathbf { S } ^ { 2 }}$ & Triadimefon & $1: 1$ & 0.64 & 1.69 & 9.39 & N/A \\
\hline $\mathbf{1 0 1} \mathbf{b S}^{\mathbf{2}}$ & Cyproconazole & $1: 1$ & 0.55 & 1.26 & 10.75 & N/A \\
\hline $\mathbf{1 0 1} \mathbf{C S}^{\mathbf{2}}$ & Tebuconazole & $1: 1$ & 0.54 & 1.52 & 8.56 & N/A \\
\hline
\end{tabular}

${ }^{1}$ (F=fungicide, $\mathrm{P}=$ =polymer), ${ }^{2}(\mathrm{~S}=$ sonicated for $\mathbf{2} \mathrm{hrs}$ prior to sampling)

Once the fungicides were successfully incorporated into the $C A B$ particles using the optimised phase inversion procedure, the final step was to analyse the encapsulation efficiency of these formulations, which was calculated using data acquired from gas chromatography (GC) of both the supernatant and the final particle formulations.

\subsubsection{Encapsulation efficiency}

Encapsulation efficiency of particle formulations were calculated by two different methods; measuring the amount of active compound in the supernatant and measuring the amount of active compound in the particles themselves. This was carried out using two different spectroscopic techniques; gas chromatography with flame ionisation detection (GC-FID) and quantitative ${ }^{1} \mathrm{H}$ NMR.

\subsubsection{1 ${ }^{1} \mathrm{H}$ NMR method}

${ }^{1} \mathrm{H}$ NMR was used to quantify the ratio of polymer to fungicide in the particles. This was achieved by dissolving known amounts (10mg) of the fungicide and the cellulose polymer in deuterated chloroform in a 1:1 mass ratio. ${ }^{1} \mathrm{H}$ NMR spectra were recorded and the peaks corresponding to the fungicide and the cellulose polymer were integrated. The ratio of these integrations (after accounting for the number of protons they represent) gave the ratio of the two components in a 1:1 mass ratio. The data collected from the ${ }^{1} \mathrm{H}$ NMR spectra is tabulated below in Table 11. 
Table 11: ${ }^{1} \mathrm{H}$ NMR analysis of polymer to fungicide mass ratio in bulk samples

\begin{tabular}{|c|c|c|c|c|}
\hline Sample & Fungicide & ${ }^{1}$ H NMR peak & Integration & $\begin{array}{l}\text { Calculated } \% \mathrm{~m} / \mathrm{m} \\
\text { of fungicide }\end{array}$ \\
\hline \multirow[t]{2}{*}{$101 a$} & Triadimefon & F: 7.97ppm & $F: 1.00$ & 54 \\
\hline & & P: 4.79ppm & P: 0.79 & \\
\hline \multirow[t]{2}{*}{ Test } & Triadimefon & F: 7.97ppm & $F: 1.00$ & 50 \\
\hline & & P: 4.79ppm & $P: 0.87$ & \\
\hline \multirow[t]{2}{*}{$101 b$} & Cyproconazole & F: 7.65ppm & $F: 1.00$ & 58 \\
\hline & & P: 4.79ppm & P: 2.09 & \\
\hline \multirow[t]{2}{*}{ Test } & Cyproconazole & F: 7.65ppm & $F: 1.00$ & 50 \\
\hline & & P: 4.78ppm & $P: 1.48$ & \\
\hline \multirow[t]{2}{*}{$101 c$} & Tebuconazole & F: $8.00 p p m$ & F: 1.00 & 55 \\
\hline & & P: 4.79ppm & P: 0.62 & \\
\hline \multirow[t]{2}{*}{ Test } & Tebuconazole & F: 8.00ppm & F: 1.00 & 50 \\
\hline & & P: 4.81ppm & $P: 0.84$ & \\
\hline
\end{tabular}

Particles were prepared using the phase inversion technique with a mass ratio of $1: 1$ fungicide to polymer. It was observed that the recovered and dried particles demonstrated a polymer to fungicide ratio as calculated using ${ }^{1} \mathrm{H}$ NMR that was close to $1: 1$ (Table 11). Between 20-29\% more of the fungicide use in the preparation was present in the particles produced giving an encapsulation efficiency of approximately $120 \%$ and an $\mathrm{m} / \mathrm{m}$ ratio of approximately $58 \%$. This clearly results from the greater insolubility of the active ingredient compared to the polymer in the solvent systems used. Thus the fungicide either precipitated before or after the polymer as the water concentration increased in the emulsion.

\subsubsection{GC-FID method}

GC-FID was used to quantify the exact amount of fungicide in the samples and in their respective supernatant mixtures. First, a standard curve of each fungicide was calculated using a known concentration of the fungicide, then a serial dilution was done using $1 \mathrm{~mL}$ of the known concentration and $9 \mathrm{~mL}$ of the internal standard mixture (dibutyl phthalate in acetone at $0.4 \mathrm{mg} / \mathrm{mL}$ ). The results of the serial dilutions on the GC-FID were graphed to give the standard curves (Figure 72). The dibutyl phthalate was used to provide a reference retention time for the fungicides in the GC-FID analysis. 
2000000 $R^{2}=1.00$

1800000

1600000

1400000

1200000

选 1000000

800000

600000

400000

200000

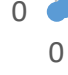

$0.05 \quad 0.1$

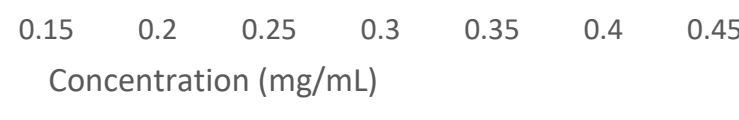

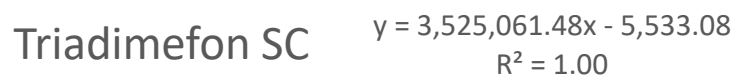

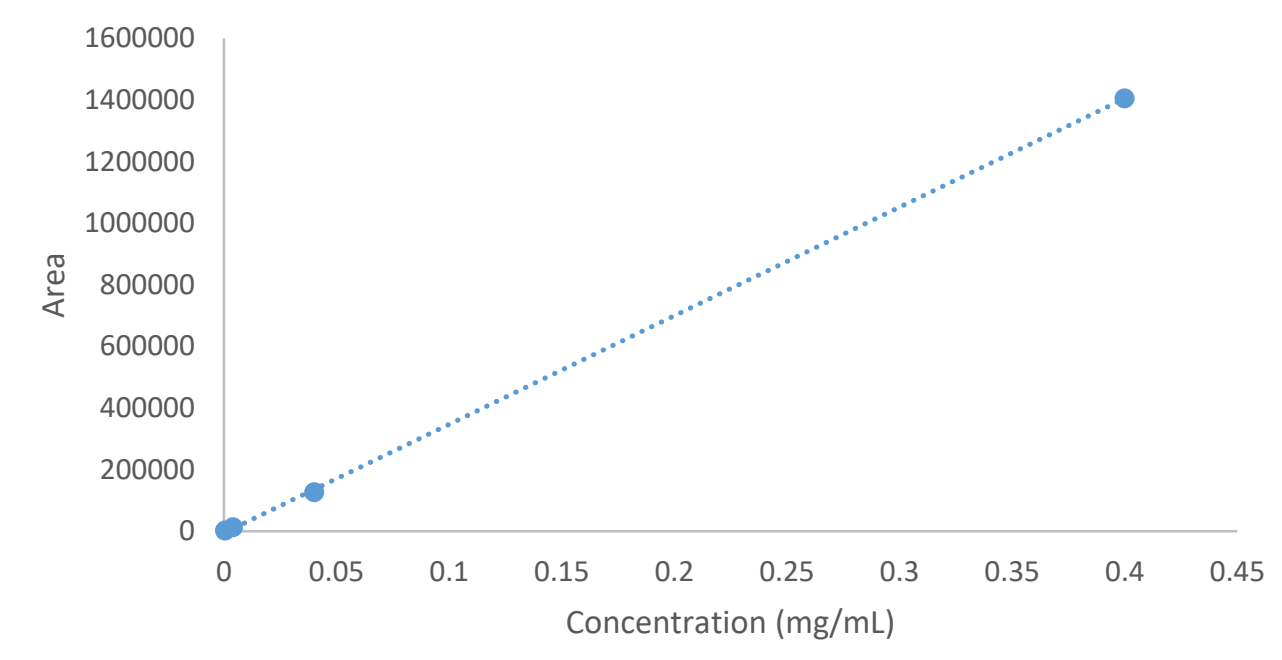

Figure 72: Standard curves for fungicides; Cyproconazole (top) and Triadimefon (bottom)

A $0.5 \mathrm{~mL}$ aliquot of the supernatants that were collected from the preparation of particles and was placed into a GC sample vial, then charged with $0.5 \mathrm{~mL}$ aliquot of the internal standard solution. The amount of fungicide present in the supernatant was calculated from the response factor of integrated areas of the peaks assigned to the fungicide. To test the particles themselves, dried purified particles of known mass $(10 \mathrm{mg})$ were suspended in acetone $(25 \mathrm{~mL}, 0.4 \mathrm{mg} / \mathrm{mL})$. A $0.5 \mathrm{~mL}$ aliquot of this solution was added to a GC sample vial along with a $0.5 \mathrm{~mL}$ aliquot of the internal standard solution and the GC-FID recorded. It was observed that the fungicide Tebuconazole was acetone insoluble, so this fungicide was not analysed for this data-set. The data collected for samples 101a and 101b are summarised in Table 12 and Table 13. 
Table 12: GC-FID data for samples 101b and 101a

\begin{tabular}{llll}
\hline Sample & Fungicide & Area & $\begin{array}{l}\text { Calculated } \\
\text { concentration }(\mathbf{m g} / \mathbf{m L})\end{array}$ \\
\hline 101b (supernatant) & Cyproconazole & 1736891 & 0.732 \\
\hline 101b (particles) & Cyproconazole & 250435 & 0.106 \\
\hline 101a (supernatant) & Triadimefon & 603632 & 0.346 \\
\hline 101a (particles) & Triadimefon & 204067 & 0.118 \\
\hline
\end{tabular}

Table 13: Calculated data encapsulation efficiency data for samples 101b and 101a

\begin{tabular}{llllll}
\hline Sample & $\begin{array}{l}\text { Mass of } \\
\text { fungicide } \\
\text { before reaction } \\
\text { (g) }\end{array}$ & $\begin{array}{l}\text { Mass of fungicide } \\
\text { in supernatant } \\
(\mathbf{m g})\end{array}$ & $\begin{array}{l}\text { Calculated } \\
\text { \%m/m of } \\
\text { fungicide in } \\
\text { particles }\end{array}$ & $\begin{array}{l}\text { Mass of } \\
\text { fungicide in } \\
\text { particles (mg) }\end{array}$ & $\begin{array}{l}\text { \% fungicide } \\
\text { incorporated }\end{array}$ \\
\hline $101 \mathrm{~b}$ & 2.5 & 330 & 26 & 792 & 32 \\
\hline $101 \mathrm{a}$ & 2.5 & 156 & 29 & 522 & 21 \\
\hline
\end{tabular}

The supernatant results show the mass of the fungicide in the total volume of supernatant $(450 \mathrm{~mL})$ collected from a single batch sample. To find this mass, the area value given by the GC-FID was substituted into the equation generated from the corresponding standard curve. This gave the concentration of the fungicide in the sample vial, of which there was $0.5 \mathrm{~mL}$ out of the total $1 \mathrm{~mL}$ volume, thus the concentration generated from the equation was doubled to account for this. This concentration was then multiplied by the total volume of supernatant collected, which was $450 \mathrm{~mL}$ for both samples and this gave the total mass of fungicide in the supernatant. The calculated mass of fungicide was found to be $330 \mathrm{mg}$ and $156 \mathrm{mg}$ for samples $101 \mathrm{~b}$ and $101 \mathrm{a}$ respectively.

To calculate the percentage of fungicide incorporated into the particles by GC-FID, the area value given by the GC-FID was substituted into the equation from the corresponding standard curve. This again gave the concentration of the fungicide in the sample vial, which again was doubled as only half the $1 \mathrm{~mL}$ solution in the GC vial was the particle solution. This concentration was then multiplied by the total volume of the volumetric flask of $25 \mathrm{~mL}$ to give the mass of fungicide in this solution. The volumetric flask was charged with particles $(10 \mathrm{mg})$ permitting $\% \mathrm{~m} / \mathrm{m}$ of fungicide to particle to be calculated. To find the percentage of fungicide incorporated into the particle, the mass in the supernatant was subtracted from that used to prepare the particles. The calculated $\% \mathrm{~m} / \mathrm{m}$ were found to be; $26 \% \mathrm{~m} / \mathrm{m}$ and $29 \% \mathrm{~m} / \mathrm{m}$ for samples $101 \mathrm{~b}$ and $101 \mathrm{a}$ respectively. 
The percentage of fungicide found to be in the particles by GC-FID was approximately $50 \%$ of the value calculated by ${ }^{1} \mathrm{H}$ NMR method. This discrepancy may have resulted from:

- insolubility of the fungicide in the solvent system used

- inefficient extraction of the fungicide from the particle

- inhomogeneity during the extraction processes.

The $\% \mathrm{~m} / \mathrm{m}$ of fungicide was also calculated by our industry partners Lonza and found to contain fungicide at; $50.8 \% \mathrm{~m} / \mathrm{m}, 41.1 \% \mathrm{~m} / \mathrm{m}$ and $44.8 \% \mathrm{~m} / \mathrm{m}$ for samples $101 \mathrm{a}, 101 \mathrm{~b}$ and $101 \mathrm{c}$, respectively. These values show the percentage of fungicide in the samples to be below $50 \%$ except for sample 101a. The numbers they found were much closer to the numbers generated by the ${ }^{1} \mathrm{H}$ NMR method. The ${ }^{1} \mathrm{H}$ NMR generated mass ratios put the fungicide at a percentage of $58 \%$ to $45 \%$. Given that all the $\% \mathrm{~m} / \mathrm{m}$ values differ between methods, and the aforementioned observation of the poor solubility of Tebuconazole in acetone, this strongly suggests the discrepancy is due to solubility issues.

\subsection{Biocide testing}

The biocide testing for the wood preservative that has been developed and produced on a small scale in this project was carried out by industrial partners Lonza. The testing that was done on the particle formulations followed the AWPC guidelines. Each of the formulations sent to Lonza had encapsulated one of the three fungicides that were used in this project. The tests were set up with the particle formulations included into plywood glue and then the small sections of plywood were glued together to form the test blocks (Figure 73).

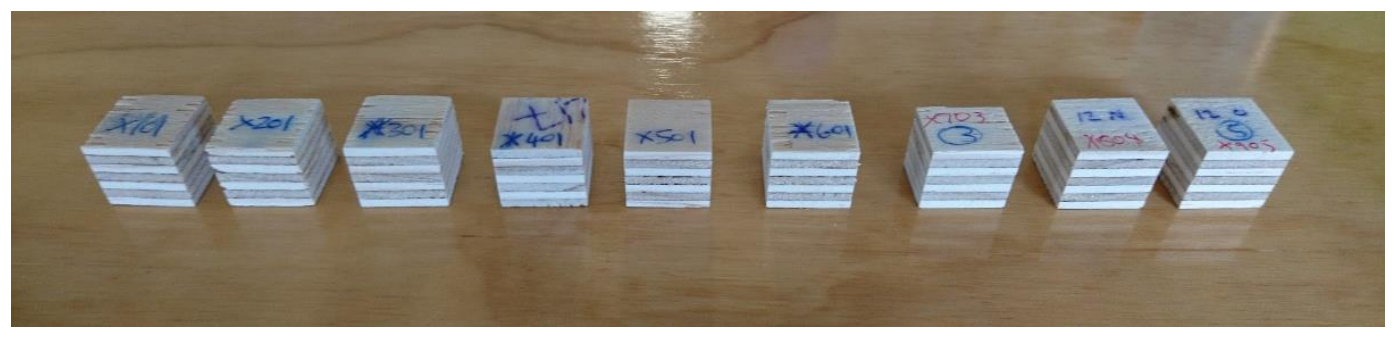

Figure 73: Plywood blocks containing particle formulations in glue

The next step was to vacuum dry the blocks to remove contaminants. Here the blocks were weighed; this gives the initial weight of the blocks that would later be compared to the weight after the fungi have degraded the wood. Once this was done, the blocks were placed into the jars that would later be inoculated with the various wood fungi. These jars were then placed into a constant humidity chamber (at approximately $75 \%$ humidity) to allow the fungi to grow (Figure 74). The test procedure required testing against various wood fungi; Coniophora puteana, Serpula lacrymans, Fomitopsis lilacino-gilva, Antrodia xantha, Tyromyces palustris (found in Japan) and Pycnoporus coccineus. 


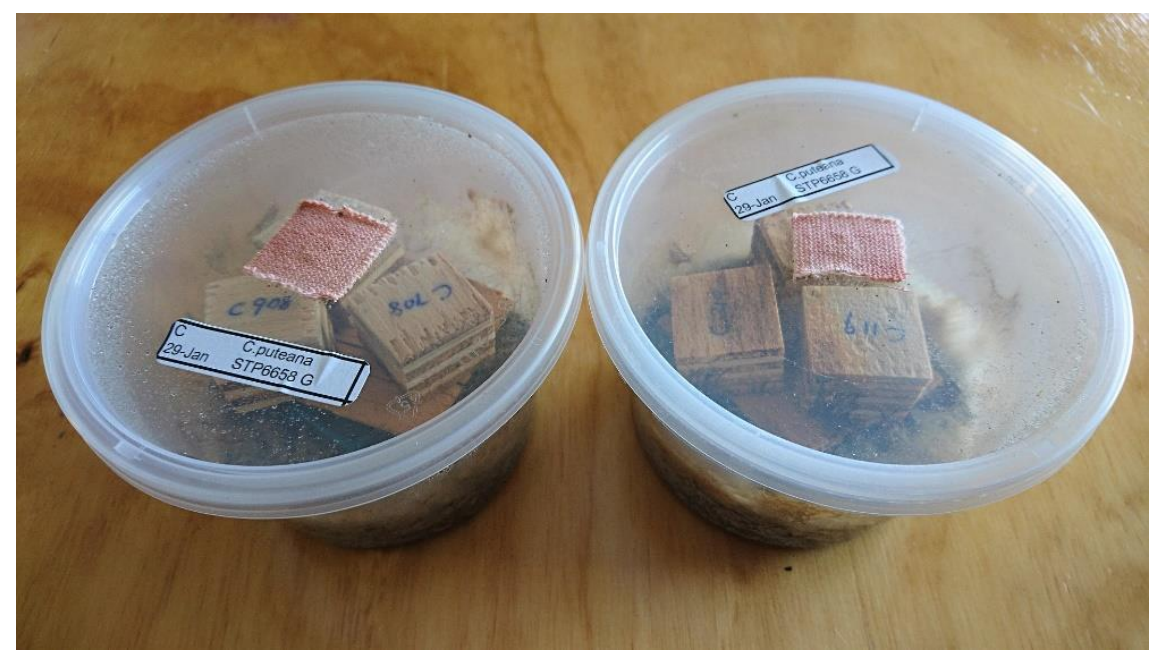

Figure 74: Jars inoculated with wood fungi Coniophora puteana, containing plywood blocks with encapsulated fungicide present in the plywood glue

The weight loss data for the samples analysed by Lonza will be assessed at the experiment completion and is ongoing.

\section{Conclusions}

A wood preserving formulation was developed with the intention of preventing wood degrading pests from proliferating without the potential for the formulation to become an environmental hazard. This was achieved by the encapsulation of known wood fungicidal agents by renewable cellulosic derivatives. The analysis of the current literature showed that the enzymes responsible for the degradation of wood by wood fungi could degrade cellulosic derivatives with relative efficiency, leading to the development of a particle forming technique that could encapsulate an active chemical agent. The methods that were trialled were: double emulsions, solvent diffusion by dialysis membrane, and phase inversion emulsification.

Phase inversion emulsification was found to be the most reproducible and generated particles that were hollow and had favourable smooth morphology. In the initial experiments, the particle size distribution obtained gave particle sizes above that of the target size of $1 \mu \mathrm{m}$. Thus, optimisation of the particle size was necessary and this was achieved by manipulation of key factors; addition time of the aqueous phase, surfactant concentration and salt concentration. These manipulations led to a bimodal distribution of particle sizes, with the majority of the particles below $1 \mu \mathrm{m}$. It was concluded 
that this bimodal distribution was due to both catastrophic and transitional phase inversion mechanisms occurring simultaneously.

With an appropriate particle size distribution and morphology achieved, the active chemical agents were incorporated; Triadimefon, Cyproconazole and Tebuconazole. On incorporation of these fungicides into the formulation it was found to have a limited effect on the particle size distribution and morphology. Once the particles loaded with active ingredient were prepared, the ratio of polymer to fungicide in the particles was analysed using two methods and compared to our industry collaborators analyses which was consistent with a high level of fungicide incorporation.

Finally, understanding the fungicide loaded particles were sent to industrial partners Lonza for biocide analysis. Variance in the level of fungicide present in the particles was observed for the different techniques used and would form the basis for future study. However, all data supports the incorporation of fungicide at $>25 \% \mathrm{w} / \mathrm{w}$ in the particles so produced.

Future work will be directed by the results from the fungicidal testing as to the efficacy of the cellulosic derived particles. Additional experiments into the encapsulation efficiency of the formulation along with testing the incorporation of insecticide compounds would be advantageous. Finally, the rate and nature of the decomposition of the cellulosics and their release characteristics would greatly benefit the future application of this technology.

\section{Experimental}

\subsection{Methods}

\subsubsection{Scanning electron microscopy}

The scanning electron microscopy experiments were carried out on a FEI Quanta 450 SEM. All samples were prepared by evaporation of a dispersion of particles in water onto an SEM stub, then coated with $\mathrm{Pd} / \mathrm{Au}$ before analysis. SEM conditions were the same for all samples; working distance $15 \mathrm{~mm}$, electron accelerating voltage of $20 \mathrm{kV}$ and the secondary electron detector was the primary detector used for all samples.

\subsubsection{Dynamic light scattering}

The particle size analysis was carried out on a Mastersizer 3000 dynamic light scattering machine. Deionised water was used as the dispersion media, and each sample was sonicated for 3 minutes, to 
eliminate sedimentation before analysis. Obscuration percentage for each sample was kept between $4-8 \%$ for each run and the refractive index of 1.41 was used to calculated particle sizes. Test time was 10 seconds with 10 second pauses in between tests. Between samples the lines were washed 3 times to ensure no contamination. Data was reported as both the average particle size (peak at the highest intensity) and as a range collected from the graphical output. Each sample was measured using three runs and the data acquired from these runs was averaged to give the distribution curves. Both sizing machines find the particle size using the same dynamic light scattering method. Where the Mastersizer 3000 used the refractive index of 1.47 and the Zetasizer used the refractive index of 1.32. Light scattering is detected at certain angles and the correlation of this light scattering with time is used to determine the diffusion coefficient of suspended particles. From this, given the viscosity of the solvent, the size of the scattering particles can be accurately determined.

\subsubsection{Infrared spectroscopy}

Infrared spectra (IR) were recorded on a Perkin Elmer Spectrum One instrument in a frequency range from $4000 \mathrm{~cm}^{-1}$ to $50 \mathrm{~cm}^{-1}$ using ATR (diamond) technology.

\subsubsection{Nuclear magnetic resonance spectroscopy}

Nuclear magnetic resonance (NMR) spectra were recorded on a Bruker Avance III $500 \mathrm{MHz}$ spectrometer. Each sample $(15 \mathrm{mg})$ was dissolved in the appropriate deuterated solvent $(0.7 \mathrm{~mL})$ in a $5 \mathrm{~mm}$ NMR tube. The spectra were collected at $25{ }^{\circ} \mathrm{C}$ and 1-dimensional ${ }^{1} \mathrm{H}$ and ${ }^{13} \mathrm{C}$ NMR were measured. Spectra were referenced to residual; $\mathrm{CHCl}_{3}{ }^{1} \mathrm{H}(7.26 \mathrm{ppm})$, DMSO ${ }^{1} \mathrm{H}(2.50 \mathrm{ppm}), \mathrm{HDO}{ }^{1} \mathrm{H}$ (4.79ppm) and the centre of $\mathrm{CDCl}_{3}\left({ }^{13} \mathrm{C} ; 77.00 \mathrm{ppm}\right)$. Polymer samples had broad peaks, these are shown in the experimental as $b=$ broad in ${ }^{1} \mathrm{H}$ NMR peak descriptions.

\subsubsection{Gas chromatography}

The gas chromatography experiments were carried out on an Agilent 6890N Network Gas Chromatograph. The flame ionisation detector was used for each experiment and the method and operating procedures are shown in the relevant experimental.

\subsubsection{Differential scanning calorimetry}

Glass transitions were determined using a Mettler Tolededo DSC1 model STAR equipped with an autosampler. Samples (0.2-2 $\mathrm{mg}$ ) were sealed in (punctured) aluminium crucibles and the heat flow measured with respect to a reference (unpunctured) crucible. The operating conditions were a temperature ramp from $-30^{\circ} \mathrm{C}$ to $180^{\circ} \mathrm{C}$ at $10^{\circ} \mathrm{C} / \mathrm{min}$ under a constant stream of dry nitrogen. Samples were cooled at the same rate and this cycle repeated twice more. Glass transitions were defined as 
the midpoint between the two inflection points observed in the glass-to rubber transition of the second and third increasing-temperature runs.

\subsection{Interfacial polymerisation}

The experiments using interfacial polymerisation were conducted using two different processes; the first was blank particle formulations that did not have any additive incorporated and the second with additives incorporated. The glass transition temperatures $\left(T_{\mathrm{g}}\right)$ were calculated using differential scanning calorimetry (DSC) (shown in Table 3 of the results discussion for clarity). Yields were not calculated for these experiments as the physical and morphological data was the primary goal.

\subsubsection{Blank particles}

To a sample vial $(15 \mathrm{~mL})$ was added a pre-prepared aqueous phase that contained PVA and sodium lignosulfonate $(5 \mathrm{w} / \mathrm{v} \%, 1 \mathrm{w} / \mathrm{v} \%, 8 \mathrm{~mL})$. The organic phase was prepared in a separate sample vial $(15 \mathrm{~mL})$, comprising polymeric diisocyanate $(0.06 \mathrm{~mL})$ and aromatic $200(2 \mathrm{~mL})$. The organic phase was added to the aqueous phase and shaken vigorously to form an oil in water emulsion. The reaction vessel was then heated to $60^{\circ} \mathrm{C}$ in a water bath, and sheer introduced (T8 Ultra turrax at max RPM of $30,000 \mathrm{rpm}$ for $2-5 \mathrm{~min})$. A solution of DETA $\left(0.4 \mathrm{~mL}\right.$ in $\left.2 \mathrm{~mL} \mathrm{of} \mathrm{H}_{2} \mathrm{O}\right)$ was added dropwise and the reaction was left for 2-5min under sheer. A precipitate formed, and the particles were separated by centrifugation, then filtered on a cellulose acetate filter paper $(0.2 \mu \mathrm{m}$ cut off) supported on a glass frit and washed with water $(10 \mathrm{~mL})$ to purify. This generated microparticles $12 \mathrm{~b}$.

\subsubsection{Particles with cellulose derivative}

To a sample vial $(15 \mathrm{~mL})$ was added a pre-prepared aqueous phase containing PVA and sodium lignosulfonate $(5 \mathrm{w} / \mathrm{v} \%, 1 \mathrm{w} / \mathrm{v} \%, 8 \mathrm{~mL})$. Cellulose derivative* $(0.114 \mathrm{~g})$ was dissolved into this solution. The organic phase was prepared in a separate sample vial $(15 \mathrm{~mL})$, to which was added polymeric diisocyanate $(0.06 \mathrm{~mL})$ and aromatic $200(2 \mathrm{~mL})$. The organic phase was added to the aqueous phase and shaken vigorously to form an oil in water emulsion. The reaction vessel was then heated to $60^{\circ} \mathrm{C}$ in a water bath, and sheer introduced (T8 Ultra turrax at max RPM of 30,000rpm for 2-5min). A solution of DETA $\left(0.4 \mathrm{~mL}\right.$ in $2 \mathrm{~mL}$ of $\left.\mathrm{H}_{2} \mathrm{O}\right)$ was added dropwise and reaction was left for $2-5 \mathrm{~min}$ and sheer. A precipitate formed, and the particles were separated by centrifugation, then filtered on a cellulose acetate filter paper $(0.2 \mu \mathrm{m}$ cut off) on a glass frit and washed with water to purify. This generated microparticles $18 \mathrm{a}$ and $16 \mathrm{~b} .{ }^{*}$ Cellulose acetate (water soluble) and methyl cellulose 


\subsection{Cellulose acetate deacetylation}

The synthesis of water soluble cellulose acetates was carried out using two different selective deacetylation methods as described below. The products were analysed by ${ }^{1} \mathrm{H}$ NMR and DSC, which were used to find the DS and $\mathrm{T}_{\mathrm{g}}$ of the product respectively.

\subsubsection{Acid treatment}

Cellulose acetate $(2 \mathrm{~g}, 398-3$ and $320-\mathrm{S})$ was dissolved in a mixture of methanol $(5.2 \mathrm{~mL})$ and acetic acid $(14.8 \mathrm{~mL})$ in a round bottom flask and stirred for an extended period to effect dissolution (8 hours). Once dissolved, $\mathrm{H}_{2} \mathrm{SO}_{4}(3 \mathrm{M}, 0.208 \mathrm{~mL})$ was added to the reaction vessel and the resulting solution was heated (reflux, 18hrs). The solution was cooled to RT, then sodium acetate $\left(2.45 \mathrm{~g}\right.$ in $20 \mathrm{~mL} \mathrm{H}_{2} \mathrm{O}$ ) was added to the reaction mixture, then a further portion of water $(30 \mathrm{~mL})$ was added and the solution became clear. The resulting mixture was then co-evaporated (with ethanol) to dryness to yield water soluble cellulose acetate. The white solid was then dissolved and dialysed (3000 MW cut off membrane) to purify, then freeze dried overnight. ${ }^{1} \mathrm{H} N M R\left(\mathrm{D}_{2} \mathrm{O}, 500 \mathrm{MHz}\right): \delta 4.62(\mathrm{~s}), 4.51(\mathrm{sb}), 4.28$ (sb), $3.96(\mathrm{mb}), 3.81(\mathrm{sb}), 3.63(\mathrm{mb}) 3.34(\mathrm{sb}), 2.7(\mathrm{~s}), 2.12(\mathrm{~m}), 1.91(\mathrm{~s})$; DSC (Tg): 97º; yield: 79\%; DS: 0.56

\subsection{2 lodine methanol treatment}

Cellulose acetate $(100 \mathrm{mg}, 398-3$ and $320-S)$ was dissolved in DMSO $(1 \mathrm{~mL})$, and with $\mathrm{I}_{2}$ dissolved in methanol (250mg in $5 \mathrm{~mL}$ ). Both solutions were added together in round bottom flask and heated $\left(80^{\circ} \mathrm{C}\right.$ for 24 hours). The reaction was quenched with a solution of $\mathrm{Na}_{2} \mathrm{~S}_{2} \mathrm{O}_{3}$ (1.5g in $20 \mathrm{~mL}$ water), causing a precipitate. The solid was freeze dried to purify to yield water soluble depolymerised cellulose acetate. ${ }^{1} \mathrm{H}$ NMR ( $\left.\mathrm{D}_{2} \mathrm{O}, 500 \mathrm{MHz}\right): \delta 7.70(\mathrm{~m}), 5.11(\mathrm{sb}), 4.72(\mathrm{sb}), 4.54(\mathrm{sb}), 4.35(\mathrm{sb}), 4.07$ (sb), $3.81(\mathrm{~m}), 2.11(\mathrm{sb}), 2.05(\mathrm{sb}), 1.99(\mathrm{sb}), 1.92(\mathrm{sb}) ;$ yield: 61\%

\subsection{Dialysis method}

The particle forming process using solvent diffusion by dialysis was conducted by varying two parameters of the formulation. These were the polymer concentration and the dye concentration, both of which were calculated as relative to the volume of organic solvent used. The dye used in all the experiments was Sudan black B, and samples 14a and 14b were formulated without the inclusion of the dye. Physical data associated with the different samples made is shown in Table 14.

General procedure:

Cellulose acetate (40mg, CA 398-3) and the Sudan black B (10mg) was dissolved in DMAc (10mL). The resulting solution was loaded into a cellulose dialysis membrane (3500 MWCO) and placed in a beaker 
$(500 \mathrm{~mL})$ of DI water $(300 \mathrm{~mL})$. The water was stirred and exchanged every 3 hours over 15 hours. The resulting suspension was centrifuged $(4000 \mathrm{rpm}, 10 \mathrm{~min}$ ) three times and washed with DI water (in triplicate). The suspension was then freeze dried to purify.

Table 14: Experimental data for formulations using the dialysis method

\begin{tabular}{|c|c|c|c|c|c|c|}
\hline $\begin{array}{l}\text { Sample } \\
\text { number }\end{array}$ & Polymer & $\begin{array}{l}\text { Dye } \\
\text { concentration } \\
(\mathrm{mg} / \mathrm{mL})\end{array}$ & $\begin{array}{l}\text { Polymer } \\
\text { concentration } \\
(\mathrm{mg} / \mathrm{mL})\end{array}$ & $\begin{array}{l}\text { Particle size } \\
\text { Range }(\mu \mathrm{m})\end{array}$ & Morphology & Observations \\
\hline $6 a$ & CA-398-3 & N/A & 4 & $0.35-1$ & $\begin{array}{l}\text { Small non- } \\
\text { spherical } \\
\text { particles, } \\
\text { appear to be } \\
\text { solid }\end{array}$ & \\
\hline $12 a$ & CA-398-3 & 1 & 2 & $0.4-0.85$ & $\begin{array}{l}\text { Small non- } \\
\text { spherical } \\
\text { particles, } \\
\text { appear to be } \\
\text { solid }\end{array}$ & \\
\hline $12 b$ & CA-398-3 & 1 & 4 & $0.45-1.1$ & $\begin{array}{l}\text { Small non- } \\
\text { spherical } \\
\text { particles, } \\
\text { appear to be } \\
\text { solid }\end{array}$ & \\
\hline $14 a$ & CA-398-3 & N/A & 6 & $0.4-0.75$ & $\begin{array}{l}\text { Small non- } \\
\text { spherical } \\
\text { particles, } \\
\text { appear to be } \\
\text { solid }\end{array}$ & $\begin{array}{l}\text { DLS shows evidence } \\
\text { of agglomeration in } \\
\text { dispersion with water }\end{array}$ \\
\hline $14 b$ & CA-398-3 & N/A & 8 & $0.4-1.9$ & $\begin{array}{l}\text { Small non- } \\
\text { spherical } \\
\text { particles, } \\
\text { appear to be } \\
\text { solid }\end{array}$ & \\
\hline $16 a$ & CA-320-S & 2 & 2 & $0.5-1.9$ & $\begin{array}{l}\text { Small } \\
\text { spherical } \\
\text { particles with } \\
\text { a smooth } \\
\text { surface }\end{array}$ & $\begin{array}{l}\text { Particles appear to be } \\
\text { coalescing }\end{array}$ \\
\hline $17 a$ & CA-320-S & 2 & 12 & $0.7-1.4$ & $\begin{array}{l}\text { Small } \\
\text { spherical } \\
\text { particles with } \\
\text { a smooth } \\
\text { surface }\end{array}$ & \\
\hline $22 a$ & CA-398-3 & 2 & 12 & $0.4-0.8$ & $\begin{array}{l}\text { Particle size } \\
\text { more } \\
\text { polydispersed } \\
\text { than } 14 a\end{array}$ & $\begin{array}{l}\text { SEM shows evidence } \\
\text { of unknown particle } \\
\text { with different } \\
\text { morphology }\end{array}$ \\
\hline $22 b$ & CA-398-3 & 2 & 16 & $0.35-0.93$ & $\begin{array}{l}\text { Particle size } \\
\text { more } \\
\text { polydispersed } \\
\text { than } 14 a\end{array}$ & \\
\hline
\end{tabular}




\subsection{Double emulsions}

The particle forming experiments using double emulsion technique were carried out using two different methods. Method 1 (shown below) is a method developed for encapsulation of two active compounds by a standard double emulsion process. Method 2 (shown below) is a method that was adapted from a method that used microfluidics to form double emulsion particles. Method 1 did not generate the desired particles. Method 2 also proved to be unsuitable for the project therefore much of the physical data was not acquired after initial viewing of the particles by SEM. The results are shown in Table 15, reproduced below and this data was acquired for particles produced by method 2 only.

\subsubsection{Method 1}

General procedure:

Ethyl cellulose (300mg) was dissolved in 1:1 DMA:ACN (10mL total), and the resulting solution was added to water $(4 \mathrm{~mL})$ under stirring $(450 \mathrm{rpm}, 15 \mathrm{~min})$. A solution of liquid paraffin $(10 \mathrm{~mL})$ with Surfynol $104 \mathrm{E}(0.15 \mathrm{~mL})$ was prepared, and the primary emulsion was added to this solution under stirring (750rpm, 2 hours). Hexane $(10 \mathrm{~mL})$ was added to the solution to precipitate the droplets. The solution was then filtered and washed with hexane. No particles were recovered.

\subsubsection{Method 2}

General procedure:

Ethyl cellulose $(1047 \mathrm{mg})$ and Surfynol $104 \mathrm{E}(0.4 \mathrm{~mL})$ was dissolved in ethyl acetate $(20 \mathrm{~mL})$. A solution of DI water $(20 \mathrm{~mL})$, ethyl acetate $(0.8 \mathrm{~mL})$ and of Termul $203(0.2 \mathrm{~mL})$ was added to the ethyl acetate solution under stirring $(750 \mathrm{rpm}, 15 \mathrm{~min})$. This primary emulsion was then added to a solution of DI water $(20 \mathrm{~mL})$, ethyl acetate $(0.8 \mathrm{~mL})$ and Termul $203(0.2 \mathrm{~mL})$ under stirring (1000rpm, 1 hour). A solution of ethanol:water ( $20 \mathrm{~mL}, 1: 4$ solution) was added to precipitate the droplets. The solution was filtered and washed with 1:4 ethanol:water $(10 \mathrm{~mL})$.

Table 15: Physical data for particles generated by the double emulsion method 2

\begin{tabular}{llll}
\hline Sample number & Polymer & $\begin{array}{l}\text { Polymer } \\
(\mathrm{mg} / \mathrm{mL})\end{array}$ & $\begin{array}{l}\text { concentration } \\
(\mu \mathrm{m})\end{array}$ \\
\hline $\mathbf{8 a}$ & EC-10 & 52.35 & $2.5-5.7$ \\
$\mathbf{1 1 a}$ & EC-10 & 52.35 & $2.7-6.1$ \\
\hline
\end{tabular}




\section{6 'Ouzo effect'}

The particle forming experiments utilising the process known as the 'Ouzo effect' were carried out using two different methods, differing only in the choice of solvent for the organic phase and scale. Both methods were adapted from previous literature works and the dye Sudan black B was used as a surrogate active compound. Method 1 was abandoned after multiple attempts to form particles, as all the experiments using this method resulted in forming a gel. Method 2 was also abandoned due to the polymer forming large globules during the sheer process.

\subsubsection{Method 1}

General procedure:

Ethyl cellulose $(400 \mathrm{mg})$ and Sudan black B $(30 \mathrm{mg})$ were dissolved in ethanol $(20 \mathrm{~mL})$. An aqueous solution ( $40 \mathrm{~mL}, 5 \% \mathrm{w} / \mathrm{v}$ PVA) was prepared and the ethanol solution was injected into the PVA solution under sheer (T8 Ultra Turrax, 10,000rpm). All experiments that were carried out with this method, the cellulose formed a gel and further purification was not carried out as a result.

\subsubsection{Method 2}

General procedure:

Ethyl cellulose $(830 \mathrm{mg})$ was dissolved in ethyl acetate $(10 \mathrm{~mL})$, then this solution was added to a solution of DI water (40mL) and PVA ( $800 \mathrm{mg})$ under sheer (T8 Ultra Turrax at 10,000rpm). All attempts to form particles using this method were stopped here as the polymer precipitated out as large globules and coagulated before an emulsion could be established and further purification was not completed.

\subsection{Emulsion inversion}

The particle forming experiments using a phase inversion process were adapted from a method designed to incorporate a sunscreen active compound. The general procedure (shown below) describes the literature procedure adapted for this project. The optimised procedure (shown below) describes the process that was developed over this project, and this method generated the particles that comply with the demands of this project. The last method described the process used to incorporate fungicide into the particles and was used in a scaled-up process. Three fungicides were used in these experiments; Triadimefon, Cyproconazole and Tebuconazole. The physical data acquired for the samples generated without fungicide incorporated, as described in the general and optimised methods are shown in Table 16. The physical data acquired for the samples generated with fungicide incorporated are shown in Table 17. 


\subsubsection{General procedure}

Cellulose acetate butyrate $(250 \mathrm{mg})$ was dissolved in acetone $(10 \mathrm{~mL})$. A solution of DI water $(20 \mathrm{~mL})$, $3 \% \mathrm{w} / \mathrm{w}$ PVA $(600 \mathrm{mg})$ and $20 \% \mathrm{w} / \mathrm{w} \mathrm{CaCl}_{2}(4 \mathrm{~g})$ was prepared and this solution was added to the acetone solution under sheer (T8 Ultra Turrax, 10,000rpm). Additional DI water $(60 \mathrm{~mL})$ was then added to the resulting emulsion and the solution centrifuged $(4000 \mathrm{rpm}, 10 \mathrm{~min}$ ) washed with DI water $(20 \mathrm{~mL})$. The washing was repeated a further two times. The washed suspension was then freeze dried overnight to purify. IR (ATR, $\mathrm{cm}^{-1}$ ): 2965 (C-H), 1741 (C=O), 1044 (C-O); yield: 71\%

\subsubsection{Optimised procedure}

Cellulose acetate butyrate $(250 \mathrm{mg})$ was dissolved in acetone $(5 \mathrm{~mL})$. A solution of DI water $(10 \mathrm{~mL})$, $6 \% \mathrm{w} / \mathrm{w}$ PVA $(600 \mathrm{mg})$ and $40 \% \mathrm{w} / \mathrm{w} \mathrm{CaCl}_{2}(4 \mathrm{~g})$ was prepared and this solution was added to the acetone solution over a period of 90 seconds under sheer (T8 Ultra Turrax, 10,000rpm). Additional DI water $(30 \mathrm{~mL}$ ) was then added to the resulting emulsion and this solution was centrifuged (at 4000rpm for $10 \mathrm{~min}$ ) washed with DI water $(20 \mathrm{~mL})$. The washing was repeated a further two times. The washed suspension was then freeze dried overnight to purify. IR (ATR, cm ${ }^{-1}$ ): 2965 (C-H), 1741 (C=O), 1044 (C0); yield: $68 \%$

\subsubsection{Fungicide incorporated procedure (bulk)}

Cellulose acetate butyrate $(5 \mathrm{~g})$ and fungicide ( $5 \mathrm{~g}$, Triadimefon / Cyproconazole / Tebuconazole) was dissolved in acetone $(100 \mathrm{~mL})$. A solution of DI water $(200 \mathrm{~mL}), 6 \% \mathrm{w} / \mathrm{w}$ PVA $(6 \mathrm{~g})$ and $40 \% \mathrm{w} / \mathrm{w} \mathrm{CaCl}_{2}$ $(20 \mathrm{~g})$ was prepared and this solution was added to the acetone solution over a period of 90 seconds under sheer (T8 Ultra Turrax, 10,000rpm). Additional DI water $(200 \mathrm{~mL})$ was then added to the resulting emulsion and this solution was centrifuged (at 4000rpm for $10 \mathrm{~min}$ ) washed with DI water. The washing was repeated a further two times. The washed suspension was then freeze dried overnight to purify. IR (ATR, cm ${ }^{-1}$ ): 3406 (O-H), 2970 (C-H), 1739 (C=O), 1491 (C=C), 1071 (C-O); yield: 45\%.

Table 16: Experimental data for formulations using the phase inversion method (without fungicide)

\begin{tabular}{|c|c|c|c|c|c|}
\hline $\begin{array}{l}\text { Sample } \\
\text { number }\end{array}$ & Polymer & $\begin{array}{l}\text { Concentration } \\
(\mathrm{mg} / \mathrm{mL})\end{array}$ & $\begin{array}{l}\text { Particle size } \\
\text { range ( } \mu \mathrm{m})\end{array}$ & Morphology & Observations \\
\hline $26 a$ & $\mathrm{EC}-10$ & 25 & $1.2-5.5$ & $\begin{array}{l}\text { Smooth surface, } \\
\text { polydisperse mixture }\end{array}$ & $\begin{array}{l}\text { SEM shows evidence of } \\
\text { the particles being hollow }\end{array}$ \\
\hline $27 a$ & $\mathrm{EC}-10$ & 25 & $1.5-4$ & $\begin{array}{l}\text { Rougher surface, larger } \\
\text { polydispersity }\end{array}$ & \\
\hline $32 a$ & CAB-381.01 & 25 & $1-5.5$ & $\begin{array}{l}\text { Smooth surface, some } \\
\text { particles collapsed }\end{array}$ & \\
\hline $32 b$ & CAB-551.01 & 25 & $0.8-2.7$ & Smooth particle surface & $\begin{array}{l}\text { Some evidence of } \\
\text { agglomeration }\end{array}$ \\
\hline $34 a$ & CAB-381.01 & 25 & $0.9-3$ & Rough surface & Agglomeration observed \\
\hline $35 a$ & CAB-381.01 & 50 & $0.6-2.6$ & $\begin{array}{l}\text { Smooth surface, some } \\
\text { particles collapsed }\end{array}$ & \\
\hline $35 b$ & CAB-381.01 & 75 & $0.8-3$ & $\begin{array}{l}\text { Smooth surface, } \\
\text { presence of much larger } \\
\text { particles }\end{array}$ & $\begin{array}{l}\text { Small particles surround } \\
\text { much larger ones }\end{array}$ \\
\hline
\end{tabular}




\begin{tabular}{|c|c|c|c|c|c|}
\hline $35 c$ & CAB-381.01 & 100 & $0.7-3$ & $\begin{array}{l}\text { Smooth surface, } \\
\text { presence of much larger } \\
\text { particles }\end{array}$ & $\begin{array}{l}\text { Wall thickness can be } \\
\text { observed where particles } \\
\text { have collapsed }\end{array}$ \\
\hline $36 a$ & CAB-381.01 & 25 & $0.5-4$ & $\begin{array}{l}\text { Smooth surface, more } \\
\text { monodisperse }\end{array}$ & \\
\hline $36 b$ & CAB-381.01 & 25 & $1-5.5$ & $\begin{array}{l}\text { Surface is rougher than } \\
\text { other PVA samples }\end{array}$ & $\begin{array}{l}\text { Presence of very small } \\
\text { particles }\end{array}$ \\
\hline $36 c$ & CAB-381.01 & 25 & $0.2-5.5$ & $\begin{array}{l}\text { Surface is rougher than } \\
\text { other PVA samples }\end{array}$ & $\begin{array}{l}\text { Presence of very small } \\
\text { particles }\end{array}$ \\
\hline $37 a$ & CAB-500.5 & 25 & $0.7-51.8$ & $\begin{array}{l}\text { Most particles have a } \\
\text { smooth surface, while } \\
\text { some particles have a } \\
\text { edged surfaces }\end{array}$ & \\
\hline $37 b$ & CAB-553.04 & 25 & $0.75-24$ & $\begin{array}{l}\text { Particles had very } \\
\text { smooth surface, with } \\
\text { some variation in } \\
\text { particle size }\end{array}$ & $\begin{array}{l}\text { Presence of some } \\
\text { particles that have broken }\end{array}$ \\
\hline $37 c$ & CAB-171.1 & 25 & $0.7-111$ & $\begin{array}{l}\text { Particles show a dimpled } \\
\text { surface, most of the } \\
\text { smaller particles had a } \\
\text { smooth surface }\end{array}$ & \\
\hline $38 a$ & CAB-381.01 & 15 & $0.6-98.1$ & $\begin{array}{l}\text { Most particles have a } \\
\text { smooth surface, while } \\
\text { some particles have a } \\
\text { dimpled surface }\end{array}$ & $\begin{array}{l}\text { Small particles surround } \\
\text { much larger ones }\end{array}$ \\
\hline $38 b$ & CAB-381.01 & 8 & $0.87-36.7$ & $\begin{array}{l}\text { Most particles have a } \\
\text { smooth surface, while } \\
\text { some particles have a } \\
\text { dimpled surface }\end{array}$ & \\
\hline $40 a$ & CAB-551.01 & 25 & $0.8-76$ & $\begin{array}{l}\text { Larger particles show a } \\
\text { rough surface, while } \\
\text { smaller particles are } \\
\text { smoother }\end{array}$ & $\begin{array}{l}\text { Presence of some very } \\
\text { large particles }\end{array}$ \\
\hline $40 b$ & CAB-551.01 & 50 & $0.8-185$ & $\begin{array}{l}\text { Larger particles show a } \\
\text { rough surface, while } \\
\text { smaller particles are } \\
\text { smoother }\end{array}$ & $\begin{array}{l}\text { Larger particles appear to } \\
\text { be deforming under } \\
\text { electron beam }\end{array}$ \\
\hline 40c & CAB-551.01 & 75 & $1.1-66$ & $\begin{array}{l}\text { Larger particles show a } \\
\text { rough surface, while } \\
\text { smaller particles are } \\
\text { smoother }\end{array}$ & $\begin{array}{l}\text { Appears to be a lot of } \\
\text { larger particle sizes in this } \\
\text { sample }\end{array}$ \\
\hline $40 d$ & CAB-551.01 & 100 & $1.1-67$ & $\begin{array}{l}\text { Similar morphology to } \\
\text { previous HM- } 40 \\
\text { samples, with a greater } \\
\text { number of larger } \\
\text { particles }\end{array}$ & \\
\hline $41 a$ & CAB-500.5 & 50 & $\mathrm{~N} / \mathrm{A}$ & $\begin{array}{l}\text { Sample has presence of } \\
\text { rods and stretched } \\
\text { particles, small } \\
\text { population of spherical } \\
\text { particles present }\end{array}$ & $\begin{array}{l}\text { Likely due to variation in } \\
\text { emulsion forming } \\
\text { conditions }\end{array}$ \\
\hline $41 b$ & CAB-500.5 & 75 & $\mathrm{~N} / \mathrm{A}$ & $\begin{array}{l}\text { Sample has presence of } \\
\text { rods and stretched } \\
\text { particles, small } \\
\text { population of spherical } \\
\text { particles present }\end{array}$ & \\
\hline 41c & CAB-500.5 & 100 & $\mathrm{~N} / \mathrm{A}$ & $\begin{array}{l}\text { Sample has presence of } \\
\text { rods and stretched } \\
\text { particles, small } \\
\text { population of spherical } \\
\text { particles present }\end{array}$ & \\
\hline $46 a$ & $\begin{array}{l}\text { CAB- } \\
381.01 / \text { Chitosa } \\
n\end{array}$ & 25 & $0.54-45.1$ & $\begin{array}{l}\text { Larger particles show } \\
\text { dimpling, with the } \\
\text { majority of particles } \\
\text { showing a smooth } \\
\text { surface }\end{array}$ & $\begin{array}{l}\text { Coalescence present on } \\
\text { some of the larger } \\
\text { particles }\end{array}$ \\
\hline $47 a$ & CAB-381.01 & 25 & $0.87-31$ & $\begin{array}{l}\text { Particles show a smooth } \\
\text { surface with some } \\
\text { showing dimpling }\end{array}$ & $\begin{array}{l}\text { Sample appears to have } \\
\text { regular particle sizes } \\
\text { present }\end{array}$ \\
\hline $47 b$ & CAB-381.01 & 25 & $0.98-24.5$ & $\begin{array}{l}\text { Sample show many } \\
\text { deformed particles }\end{array}$ & \\
\hline
\end{tabular}




\begin{tabular}{|c|c|c|c|c|c|}
\hline & & & & $\begin{array}{l}\text { present, common } \\
\text { morphology is smooth } \\
\text { with the smaller } \\
\text { particles }\end{array}$ & \\
\hline $47 c$ & CAB-381.01 & 25 & $0.87-24.1$ & $\begin{array}{l}\text { Majority of smaller } \\
\text { particles have a smooth } \\
\text { surface, larger particles } \\
\text { are dimpled }\end{array}$ & $\begin{array}{l}\text { Presence of many large } \\
\text { particles }\end{array}$ \\
\hline $48 a$ & $\begin{array}{l}\text { CAB- } \\
\text { 381.01/Chitosa } \\
n\end{array}$ & 25 & $\mathrm{~N} / \mathrm{A}$ & $\begin{array}{l}\text { Particle surface appears } \\
\text { rougher than in previous } \\
\text { samples, with limited } \\
\text { dimpling }\end{array}$ & \\
\hline $48 b$ & $\begin{array}{l}\text { CAB- } \\
381.01 / \text { Chitosa } \\
n\end{array}$ & 25 & $\mathrm{~N} / \mathrm{A}$ & $\begin{array}{l}\text { Particles appear smooth } \\
\text { with some non-spherical } \\
\text { particles }\end{array}$ & $\begin{array}{l}\text { Presence of some very } \\
\text { large particles }\end{array}$ \\
\hline $48 c$ & $\begin{array}{l}\text { CAB- } \\
\text { 381.01/Chitosa } \\
n\end{array}$ & 25 & $\mathrm{~N} / \mathrm{A}$ & $\begin{array}{l}\text { Particles appear smooth } \\
\text { with some non-spherical } \\
\text { particles, evidence of } \\
\text { excess PVA/polymer } \\
\text { that has precipitated out }\end{array}$ & $\begin{array}{l}\text { Presence of some very } \\
\text { large particles }\end{array}$ \\
\hline $50 a$ & CAB-381.01 & 60 & $0.83-31.5$ & $\begin{array}{l}\text { Most particles show a } \\
\text { smooth surface, with } \\
\text { some showing an } \\
\text { irregular surface } \\
\text { morphology }\end{array}$ & $\begin{array}{l}\text { Large number of particles } \\
\text { have holes on the surface }\end{array}$ \\
\hline $50 \mathrm{~b}$ & CAB-381.01 & 60 & $0.76-21.3$ & $\begin{array}{l}\text { Some surfaces are } \\
\text { rough, could be excess } \\
\text { bipyridine on surface, } \\
\text { majority are smooth }\end{array}$ & $\begin{array}{l}\text { Large number of particles } \\
\text { have holes on the surface }\end{array}$ \\
\hline $50 c$ & CAB-381.01 & 60 & $\mathrm{~N} / \mathrm{A}$ & $\begin{array}{l}\text { Some surfaces are } \\
\text { rough, could be excess } \\
\text { bipyridine on surface, } \\
\text { majority are smooth }\end{array}$ & $\begin{array}{l}\text { Some evidence of } \\
\text { agglomeration }\end{array}$ \\
\hline $58 a$ & CAB-381.01 & 25 & $0.875-35.3$ & $\begin{array}{l}\text { Particles have a smooth } \\
\text { surface, some particles } \\
\text { are coalescing }\end{array}$ & $\begin{array}{l}\text { Larger number of smaller } \\
\text { particles and less larger } \\
\text { ones in this sample }\end{array}$ \\
\hline $58 b$ & CAB-381.01 & 25 & $0.767-36.1$ & $\begin{array}{l}\text { Most particles have a } \\
\text { smooth surface, some } \\
\text { have a rougher surface, } \\
\text { coalescence present in } \\
\text { areas }\end{array}$ & $\begin{array}{l}\text { Again, larger number of } \\
\text { smaller particles, } \\
\text { evidence of particle size } \\
\text { control being exhibited }\end{array}$ \\
\hline $58 c$ & CAB-381.01 & 25 & $0.871-45.1$ & $\begin{array}{l}\text { Smaller particles have a } \\
\text { smooth surface, while } \\
\text { larger ones have a } \\
\text { dented surface }\end{array}$ & $\begin{array}{l}\text { Presence of larger } \\
\text { particles than previous } \\
\text { experiments, these are } \\
\text { surrounded by smaller } \\
\text { particles, evidence of } \\
\text { Ostwald ripening } \\
\text { occurring }\end{array}$ \\
\hline $59 a$ & CAB-381.01 & 25 & $0.45-24.5$ & $\begin{array}{l}\text { The small particles are } \\
\text { smooth and spherical, } \\
\text { some larger particles are } \\
\text { collapsed hollow } \\
\text { particles }\end{array}$ & $\begin{array}{l}\text { This series of samples had } \\
\text { great particle size } \\
\text { distribution, and this is } \\
\text { confirmed in the SEM } \\
\text { photos }\end{array}$ \\
\hline $59 b$ & CAB-381.01 & 25 & $0.35-9.8$ & $\begin{array}{l}\text { Similar morphology as } \\
\text { the previous sample }\end{array}$ & $\begin{array}{l}\text { This sample has better } \\
\text { distribution than the } \\
\text { previous sample, where } \\
\text { the majority of the } \\
\text { particles are } 1 \mu \mathrm{m}\end{array}$ \\
\hline $59 c$ & CAB-381.01 & 25 & $0.59-12.8$ & $\begin{array}{l}\text { Similar morphology as } \\
\text { the previous samples, } \\
\text { here there is more } \\
\text { polydispersity, and } \\
\text { larger particles have } \\
\text { collapsed }\end{array}$ & $\begin{array}{l}\text { This sample has more } \\
\text { polydispersity than the } \\
\text { previous two samples, } \\
\text { also coalescence is } \\
\text { present throughout } \\
\text { sample }\end{array}$ \\
\hline $62 a$ & CAB-381.01 & 50 & $0.46-24.9$ & $\begin{array}{l}\text { Particles have a smooth } \\
\text { surface, with some burst } \\
\text { particles, high } \\
\text { prevalence of } \\
\text { coalescence in the } \\
\text { sample }\end{array}$ & $\begin{array}{l}\text { This sample has } \\
\text { somewhat two } \\
\text { populations of particle } \\
\text { sizes, where the majority } \\
\text { are in the } 10-\mu \mathrm{m} \text { size }\end{array}$ \\
\hline
\end{tabular}




\begin{tabular}{|c|c|c|c|c|c|}
\hline $62 c$ & CAB-381.01 & 50 & $0.52-35.1$ & $\begin{array}{l}\text { Majority of smaller } \\
\text { particles are smooth and } \\
\text { spherical; many larger } \\
\text { particles are collapsed } \\
\text { and high prevalence of } \\
\text { coalescence in this } \\
\text { sample }\end{array}$ & $\begin{array}{l}\text { This sample again has two } \\
\text { different population of } \\
\text { particle sizes, the smaller } \\
\text { particles are the minority } \\
\text { as the particles above } 10 \\
\mu \mathrm{m} \text { are most prevalent }\end{array}$ \\
\hline 64a & CAB-381.01 & 50 & $\begin{array}{l}(0.45-1.25),(58- \\
200)\end{array}$ & $\begin{array}{l}\text { Particles of 500nm are } \\
\text { present in high number } \\
\text { and are smooth in } \\
\text { appearance, there is } \\
\text { presence of small rod } \\
\text { like structures, which } \\
\text { most likely is PVA }\end{array}$ & $\begin{array}{l}\text { This sample has a } \\
\text { significant bimodal } \\
\text { distribution of particle } \\
\text { sizes, the smaller particles } \\
\text { show good morphology, } \\
\text { where the larger ones are } \\
\text { much rougher in } \\
\text { appearance }\end{array}$ \\
\hline $64 b$ & CAB-381.01 & 50 & $\begin{array}{l}(0.46-1.27),(40- \\
211)\end{array}$ & $\begin{array}{l}\text { Smaller particle sizes } \\
\text { here show a smooth and } \\
\text { spherical morphology, } \\
\text { and appear to exhibit no } \\
\text { coalescence }\end{array}$ & $\begin{array}{l}\text { This sample shows a } \\
\text { significant bimodal } \\
\text { distribution of particle } \\
\text { sizes, in this sample this is } \\
\text { more significant that } \\
\text { sample 64a, shown by } \\
\text { DLS data }\end{array}$ \\
\hline $64 c$ & CAB-381.01 & 50 & $\begin{array}{l}(0.4-0.76),(45- \\
185)\end{array}$ & $\begin{array}{l}\text { Very small particles are } \\
\text { present in this sample, } \\
\text { and show good smooth } \\
\text { morphologies with } \\
\text { limited coalescence }\end{array}$ & $\begin{array}{l}\text { This sample shows a } \\
\text { bimodal distribution of } \\
\text { particle sizes, here the } \\
\text { majority of the bulk is at } \\
\text { the smaller size range, } \\
\text { particularly compared to } \\
\text { the previous two samples } \\
\text { in the series }\end{array}$ \\
\hline
\end{tabular}

Table 17: Experimental data for formulations using the phase inversion method (with fungicide)

\begin{tabular}{|c|c|c|c|c|c|c|}
\hline $\begin{array}{l}\text { Sample } \\
\text { number }\end{array}$ & Fungicide & $\begin{array}{l}\text { Particle } \\
\text { size range } \\
(\mu \mathrm{m})\end{array}$ & $\begin{array}{l}\text { Polymer } \\
\text { concentration } \\
(\mathrm{mg} / \mathrm{mL})\end{array}$ & $\begin{array}{l}\text { Fungicide } \\
\text { concentration } \\
(\mathrm{mg} / \mathrm{mL})\end{array}$ & Morphology & Observations \\
\hline $101 a$ & Triadimefon & $\begin{array}{l}(0.3-2.13) \\
(4.58-16.8)\end{array}$ & 50 & 50 & $\begin{array}{l}\text { Particle sizes are } \\
\text { fairly uniform } \\
\text { throughout } \\
\text { sample, the } \\
\text { majority of particle } \\
\text { have a smooth and } \\
\text { spherical } \\
\text { morphology }\end{array}$ & $\begin{array}{l}\text { A bimodal } \\
\text { distribution of } \\
\text { particle sizes is } \\
\text { observed in the } \\
\text { DLS. The majority } \\
\text { of the sample is at } \\
\text { the smaller size } \\
\text { range, which is the } \\
\text { target size. }\end{array}$ \\
\hline $101 b$ & Cyproconazole & $\begin{array}{l}(0.243- \\
1.87),(5.2- \\
35.1)\end{array}$ & 50 & 50 & $\begin{array}{l}\text { Particle sizes are } \\
\text { fairly uniform } \\
\text { throughout } \\
\text { sample, the } \\
\text { majority of particle } \\
\text { have a smooth and } \\
\text { spherical } \\
\text { morphology }\end{array}$ & $\begin{array}{l}\text { A bimodal } \\
\text { distribution of } \\
\text { particle sizes is } \\
\text { observed in the } \\
\text { DLS. The majority } \\
\text { of the sample is at } \\
\text { the smaller size } \\
\text { range, which is the } \\
\text { target size. }\end{array}$ \\
\hline $101 c$ & Tebuconazole & $\begin{array}{l}(0.314- \\
16.42)\end{array}$ & 50 & 50 & $\begin{array}{l}\text { Particle sizes are } \\
\text { fairly uniform } \\
\text { throughout } \\
\text { sample, the } \\
\text { majority of particle } \\
\text { have a smooth and } \\
\text { spherical } \\
\text { morphology }\end{array}$ & $\begin{array}{l}\text { This sample does } \\
\text { not show the } \\
\text { bimodal } \\
\text { distribution of the } \\
\text { previous two } \\
\text { samples, only a } \\
\text { shoulder off the } \\
\text { main distribution. }\end{array}$ \\
\hline
\end{tabular}




\subsection{Dye labelling}

Experiments were conducted to synthesise a cellulose polymer functionalised with a visible dye. This consisted of reacting a diisocyanate with a dye that had an appropriate free functional group to react with the isocyanate. The goal was to then react the functionalised dye with a cellulose acetate butyrate. The reaction with the cellulose proved the most difficult to characterise and purify. Experiment details have been provided for reference and (even if physical data could not be recorded) in developing future directions for dye functionalisation of cellulosics.

\subsubsection{Phenyl isocyanate with benzyl amine}

A round bottom flask was purged with argon. Benzyl amine $(0.1 \mathrm{~mL})$ was added to DMSO $(1 \mathrm{~mL})$. The solution was stirred and heated $\left(55^{\circ} \mathrm{C}\right)$, then phenyl isocyanate $(0.1 \mathrm{~mL}, 1$ equiv) was then added dropwise and the reaction stirred (12hrs). The resulting solution was then precipitated into DI water (approx. $10 \mathrm{~mL}$ ) under stirring and the solid was collected by vacuum filtration and washed with water. The resulting solid was then freeze dried; ${ }^{1} \mathrm{H}_{\mathrm{NMR}}\left(\mathrm{D}^{6}\right.$-DMSO, 500Mhz): $\delta 8.52(\mathrm{~s}, 1 \mathrm{H}), 7.39(\mathrm{~m}), 7.32$ $(\mathrm{m}), 7.22(\mathrm{~m}), 6.89(\mathrm{tt}, 1 \mathrm{hz}, 7 \mathrm{hz}), 6.59(\mathrm{t}, 7 \mathrm{hz}), 4.29(\mathrm{~d}, 7 \mathrm{hz})$; IR (ATR, $\left.\mathrm{cm}^{-1}\right)$ : $3230(\mathrm{C}-\mathrm{H}), 1631(\mathrm{C}=0)$; yield: $76 \%$.

\subsubsection{Phenyl isocyanate with CAB 553.04}

CAB $553.04(100 \mathrm{mg})$ and DABCO $(10 \mathrm{mg})$ were added to a round bottom flask and purged with argon. DMSO $(1 \mathrm{~mL})$ was added and the solution stirred to dissolve the polymer. Once the polymer was in solution the flask was heated $\left(55^{\circ} \mathrm{C}\right)$ and phenyl isocyanate $(0.1 \mathrm{~mL})$ was added dropwise under stirring. The reaction was allowed to proceed over $16 \mathrm{hrs}$. The resulting solution was precipitated into DI water with stirring, and the solid was filtered washed $\left(\mathrm{H}_{2} \mathrm{O}, 10 \mathrm{~mL}\right)$ and freeze dried; ${ }^{1} \mathrm{H}$ NMR $\left(D^{6}-\mathrm{DMSO}\right.$, 500Mhz): $\delta 8.52$ (s, 1H), $7.39(\mathrm{~m}), 7.32(\mathrm{~m}), 7.22(\mathrm{~m}), 6.98(\mathrm{tt}, 2 \mathrm{hz}, 7 \mathrm{hz}), 1.51(\mathrm{~b}), 0.82(\mathrm{~b}) ;$ IR (ATR, cm 1): $3292(\mathrm{C}-\mathrm{H}), 1690$ (C=O), 1047 (C-O).

\subsubsection{IPDI with benzyl alcohol}

DABCO (10mg) in DMSO (1 $\mathrm{mL})$ was added to a round bottom flask and purged with argon, IPDI $(0.388 \mathrm{~mL})$ was added to the flask. The solution was stirred and heated to $55^{\circ} \mathrm{C}$, benzyl alcohol $(0.192 \mathrm{~mL})$ was added dropwise to the solution and heated for a further $16 \mathrm{hrs}$. The reaction was monitored by TLC and no change from starting material was observed after $24 \mathrm{hrs}$ of reaction time, no further purification was carried out.

\subsubsection{IPDI with benzyl amine}

DABCO (10mg) was added to a round bottom flask and purged with argon. IPDI $(0.388 \mathrm{~mL})$ was added to the flask. The solution was heated to $55^{\circ} \mathrm{C}$ and with stirring, benzyl amine $(0.2 \mathrm{~mL})$ was added 
dropwise. A precipitate formed within 10 seconds. The resulting solid was recovered by evaporation of excess benzylamine; ${ }^{1} \mathrm{H}$ NMR $\left(\mathrm{CDCl}_{3}, 500 \mathrm{Mhz}\right): \delta 7.33(\mathrm{~m}), 7.24,(\mathrm{~m}), 7.20(\mathrm{~m}), 5.59(\mathrm{t}, 6 \mathrm{hz}), 5.47(\mathrm{t}$, $6 \mathrm{hz}), 5.22(\mathrm{~m}), 5.07(\mathrm{~m}), 4.94(\mathrm{~d}, 8 \mathrm{hz}), 4.77(\mathrm{t}, 6 \mathrm{hz}), 4.68(\mathrm{t}, 7 \mathrm{hz}), 4.32(\mathrm{~m}), 4.21(\mathrm{~m}), 3.93(\mathrm{~m}), 3.83(\mathrm{~m})$, $3.65(\mathrm{tt}, 4 \mathrm{hz}, 12 \mathrm{hz}), 3.52(\mathrm{~m}), 3.29(\mathrm{~m}), 3.05(\mathrm{~s}), 2.97(\mathrm{~s}), 2.91-2.77(\mathrm{~m}), 1.24-.73(\mathrm{~m}), 0.69(\mathrm{~s}), 0.58(\mathrm{t}$, 13hz); IR (ATR, $\left.\mathrm{cm}^{-1}\right)$ : $3326(\mathrm{O}-\mathrm{H}), 2918(\mathrm{C}-\mathrm{H}), 2255$ (N=C=O); yield: 71\%.

\subsubsection{IPDI with CAB 553.04 (DABCO)}

DABCO (10mg) was added to a round bottom flask and purged with argon, IPDI (0.1 mL) was charged into the flask and stirred to form a homogenous solution. A homogenous solution of CAB 553.04 (100mg) in DMSO $(1 \mathrm{~mL})$ was added dropwise to the reaction vessel, before heating $\left(50^{\circ} \mathrm{C}\right.$ for $\left.15 \mathrm{hrs}\right)$. No physical changes were observed after the $15 \mathrm{hrs}$, the polymer was precipitated out with DI water and NMR showed no reaction had occurred.

\subsubsection{IPDI with CAB 553.04 (TEA)}

A round bottom flask was charged with CAB $553.04(100 \mathrm{mg})$ in THF $(1 \mathrm{~mL})$, the reaction flask was purged with argon and heated $\left(55^{\circ} \mathrm{C}\right)$. TEA $(0.5 \mathrm{~mL})$ was added, followed by the dropwise addition of IPDI $(0.3 \mathrm{~mL})$ and the reaction was allowed to proceed over $16 \mathrm{hrs}$. The resulting polymer was precipitated into DI water (approx. 10mL) and the solid was collected by filtration, then freeze dried. The product was insoluble in common solvents, no NMR was taken. IR (ATR, cm-1): 3349 (O-H), 2954 (C-H), $2253(\mathrm{~N}=\mathrm{C}=\mathrm{O}), 1746(\mathrm{C}=\mathrm{O})$, yield $41 \%$

\subsubsection{Diacid chloride with benzyl alcohol}

A round bottom flask was charged with benzyl alcohol $(0.2 \mathrm{~mL})$, pyridine $(0.08 \mathrm{~mL})$ and DMAP $(20 \mathrm{mg})$ in THF ( $2 \mathrm{~mL})$. The reaction flask was purged with argon and set cooled in ice bath. Glutaryl dichloride $(0.92 \mathrm{~mL})$ was added dropwise under stirring, and a white precipitate formed instantly. The reaction was stirred for a further $12 \mathrm{hrs}$, then the solid was filtered and the filtrate collected. No further purification was carried out as the reaction was found to have not proceeded.

\subsubsection{Diacid chloride with CAB 553.04}

A round bottom flask was charged with DMAP $(15 \mathrm{mg})$, CAB $553.04(100 \mathrm{mg})$, and pyridine $(0.08 \mathrm{~mL})$ in THF ( $2 \mathrm{~mL})$. The reaction flask was then purged with argon and glutaryl dichloride $(0.92 \mathrm{~mL})$ was added dropwise under stirring. A white precipitate was formed instantly. The reaction was stirred for a further $12 \mathrm{hrs}$. No further purification was carried out as a complex insoluble intractable solid resulted.

\subsubsection{IPDI with hydroxy-naphthoquinone}

A round bottom flask was charged with hydroxy-naphthoquinone $(100 \mathrm{mg})$ and DABCO $(10 \mathrm{mg})$ in THF $(2 \mathrm{~mL})$, the reaction flask was then purged with argon and heated $\left(60^{\circ} \mathrm{C}\right)$. IPDI $(0.372 \mathrm{~mL})$ was added 
dropwise under stirring, and the reaction was allowed to proceed for $16 \mathrm{hrs}$. The solvent was removed by rotary evaporation to form the functionalised dye 12 . An intractable gum was generated, thus no yields were found. ${ }^{1} \mathrm{H}$ NMR $\left(\mathrm{CDCl}_{3}, 500 \mathrm{Mhz}\right): \delta 8.33(\mathrm{~m}), 8.13(\mathrm{~m}), 8.01(\mathrm{~m}), 7.70(\mathrm{~m}), 7.63(\mathrm{~m})$, 7.57$7.49(\mathrm{~m}), 7.45(\mathrm{~m}), 5.45(\mathrm{t}), 5.30(\mathrm{t}), 5.17(\mathrm{t}), 5.04(\mathrm{~d}), 3.83(\mathrm{~m}), 3.69(\mathrm{q}), 3.43(\mathrm{~s}), 3.29-3.09(\mathrm{~m}), 2.96$ $(\mathrm{m}), 2.74(\mathrm{q}), 1.23(\mathrm{t}), 1.11(\mathrm{t})$

\subsubsection{IPDI with Primuline}

A round bottom flask was charged with Primuline $(50 \mathrm{mg})$ and DABCO $(10 \mathrm{mg})$ in DMSO $(1 \mathrm{~mL})$. The reaction flask was then purged with argon and heated to $60^{\circ} \mathrm{C}$. IPDI $(0.15 \mathrm{~mL}, 10$ equiv)) was added dropwise with stirring (16hrs). An intractable gum was generated, thus no yields were attained. ${ }^{1} \mathrm{H}$ NMR (D6-DMSO, 500Mhz): $\delta 7.80$ (d), 7.56 (d), 7.37 (d), 7.31 (d), $5.78(\mathrm{~m}), 3.67(\mathrm{~m}), 2.68(\mathrm{~s}), 2.40(\mathrm{~s})$, $0.94(\mathrm{~m})$; IR (ATR, cm ${ }^{-1}$ ): $3362(\mathrm{O}-\mathrm{H}), 2919$ (C-H), 2259 ( $\left.\mathrm{N}=\mathrm{C}=\mathrm{O}\right), 1637$ (C=O)

\subsubsection{CAB 553.04 with IPDI-Primuline}

A homogenous solution of CAB $553.04(100 \mathrm{mg})$ in DMSO $(1 \mathrm{~mL})$ was added to a flask containing IPDIPrimuline. The flask was purged with argon and heated to $55^{\circ} \mathrm{C}$ for $12 \mathrm{hrs}$. The resulting polymer was precipitated into DI water under stirring and the solid was freeze dried to purify. The Product could not be re-dissolved due to cross-linking. Therefore, no NMR or physical data was acquired for this product.

\subsubsection{CAB 553.04 with IPDI- hydroxyl-naphthoquinone}

A homogenous solution of CAB $553.04(100 \mathrm{mg})$ in THF $(1 \mathrm{~mL})$ was added to flask containing IPDIhydroxyl-naphthoquinone. The flask was purged with argon and heated to $55^{\circ} \mathrm{C}$ for $12 \mathrm{hrs}$. The resulting polymer was precipitated into DI water under stirring and the solid was freeze dried to purify. The Product could not be re-dissolved due to cross-linking. Therefore, no NMR or physical data was acquired for this product.

\subsection{GC analysis}

All GC analysis was carried out using Flame Ionisation Detection (FID). Experimental details for GC operation are shown in Table 18.

Table 18: SOP for formulations with Cyproconazole and Triadimefon incorporated

Parameter Value




\begin{tabular}{ll}
\hline Initial temperature, hold time & $150^{\circ} \mathrm{C}, 1 \mathrm{~min}$ \\
Final temperature, hold time & $280^{\circ} \mathrm{C}, 5 \mathrm{~min}$ \\
Rate $\left({ }^{\circ} \mathrm{C} / \mathrm{min}\right)$ & $15^{\circ} \mathrm{C}$ \\
\hline Initial carrier gas velocity & $27 \mathrm{~cm} / \mathrm{sec}$ \\
Split ratio & $1: 20$ \\
Injection volume & $1 \mu \mathrm{L}$ \\
Run time & $15 \mathrm{~min}$ \\
\hline
\end{tabular}

\subsubsection{Standard solutions}

\subsubsection{Internal standard}

Dibutyl phthalate $(100 \mathrm{mg})$ was added to a $100 \mathrm{~mL}$ volumetric flask and acetone was added up to the mark with stirring. A $1 \mathrm{~mL}$ aliquot of this solution was added to a GC sample vial for analysis.

\subsubsection{Standards}

Tradimefon (10mg) was added to a $25 \mathrm{~mL}$ volumetric flask and the internal standard solution was added up to the mark with stirring. Cyproconazole $(10 \mathrm{mg})$ was added to a $25 \mathrm{~mL}$ volumetric flask and the internal standard solution was added up to the mark with stirring. A $1 \mathrm{~mL}$ aliquot of these solutions was each added to a GC sample vial for analysis

\subsubsection{Standard curves}

A serial dilution was carried out on each of the standard solutions, by taking a $1 \mathrm{~mL}$ aliquot of the standard solution and then adding a $9 \mathrm{~mL}$ aliquot of internal standard solution. This process was carried out three times and the subsequent GC-FID was carried out. The standard curve was generated using the relative area of the peaks associated with the fungicides (shown in Figure 72).

\subsubsection{Supernatant analysis}

A $0.5 \mathrm{~mL}$ aliquot of the first supernatant wash from the particle production was added to a GC sample vial, along with $0.5 \mathrm{~mL}$ aliquot of internal standard solution was added and mixed to form a homogenous solution for GC analysis.

\subsubsection{Particle analysis}

Particles formulated with fungicides (10mg) was added to a $25 \mathrm{~mL}$ volumetric flask and acetone was added up to the mark with stirring. A $0.5 \mathrm{~mL}$ aliquot of this solution was added to a GC sample vial. A $0.5 \mathrm{~mL}$ aliquot of internal standard solution was added to the same GC sample vial and mixed to form a homogenous solution for GC analysis. 


\section{References}

1. O'SULLIVAN, A. C., Cellulose: the structure slowly unravels. Cellulose 1997, 4 (3), 173-207.

2. Gierer, J., Chemical aspects of kraft pulping. Wood Science and Technology 1980, 14 (4), 241266.

3. Jarmin, R. S., Learning by doing and competition in the early rayon industry. The RAND journal of Economics 1994, 441-454.

4. Gümüskaya, E.; Usta, M.; Kirci, H., The effects of various pulping conditions on crystalline structure of cellulose in cotton linters. Polymer degradation and stability 2003, 81 (3), 559-564.

5. Nada, A.; Dawy, M.; Salama, A., Dielectric properties and ac-conductivity of cellulose polyethylene glycol blends. Materials chemistry and Physics 2004, 84 (2-3), 205-215.

6. Schœnbein, P., II. On the discovery of gun-cotton. Philosophical Magazine Series 3 1847, 31 (205), 7-12.

7. Samios, E.; Dart, R.; Dawkins, J., Preparation, characterization and biodegradation studies on cellulose acetates with varying degrees of substitution. Polymer 1997, 38 (12), 3045-3054.

8. Williamson, J.; Graham, J.; Allman, D., The modification of cigarette smoke by filter tips. Beiträge zur Tabakforschung/Contributions to Tobacco Research 1965, 3 (3), 233-242.

9. Lonsdale, H.; Merten, U.; Riley, R., Transport properties of cellulose acetate osmotic membranes. Journal of Applied Polymer Science 1965, 9 (4), 1341-1362.

10. Fundueanu, G.; Constantin, M.; Esposito, E.; Cortesi, R.; Nastruzzi, C.; Menegatti, E., Cellulose acetate butyrate microcapsules containing dextran ion-exchange resins as self-propelled drug release system. Biomaterials 2005, 26 (20), 4337-4347.

11. Grunert, M.; Winter, W. T., Nanocomposites of cellulose acetate butyrate reinforced with cellulose nanocrystals. Journal of Polymers and the Environment 2002, 10 (1-2), 27-30.

12. Qiu, X.; Hu, S., "Smart" materials based on cellulose: a review of the preparations, properties, and applications. Materials 2013, 6 (3), 738-781.

13. Koch, W., Properties and Uses of Ethylcellulose. Industrial \& Engineering Chemistry 1937, 29 (6), 687-690.

14. Floury, J.; Desrumaux, A.; Axelos, M. A. V.; Legrand, J., Effect of high pressure homogenisation on methylcellulose as food emulsifier. Journal of Food Engineering 2003, 58 (3), 227-238.

15. Donbrow, M.; Samuelov, Y., Zero order drug delivery from double-layered porous films: release rate profiles from ethyl cellulose, hydroxypropyl cellulose and polyethylene glycol mixtures. Journal of Pharmacy and pharmacology 1980, 32 (1), 463-470.

16. Pillai, O.; Panchagnula, R., Polymers in drug delivery. Current opinion in chemical biology 2001, $5(4), 447-451$.

17. El-Sakhawy, M.; Kamel, S.; Salama, A.; Sarhan, H.-A., Carboxymethyl Cellulose Acetate Butyrate: A Review of the Preparations, Properties, and Applications. Journal of Drug Delivery 2014, 2014, 6.

18. Goodell, B.; Qian, Y.; Jellison, J., Fungal Decay of Wood: Soft Rot-Brown Rot-White Rot. In Development of Commercial Wood Preservatives, American Chemical Society: 2008; Vol. 982, pp 9-31. 19. Bari, E.; Nazarnezhad, N.; Kazemi, S. M.; Tajick Ghanbary, M. A.; Mohebby, B.; Schmidt, O.; Clausen, C. A., Comparison between degradation capabilities of the white rot fungi Pleurotus ostreatus and Trametes versicolor in beech wood. International Biodeterioration \& Biodegradation 2015, 104, 231-237.

20. Su, N.-Y.; Scheffrahn, R. H., Termites as Pests of Buildings. In Termites: Evolution, Sociality, Symbioses, Ecology, Abe, T.; Bignell, D. E.; Higashi, M., Eds. Springer Netherlands: Dordrecht, 2000; pp 437-453.

21. Warnecke, F.; Luginbühl, P.; Ivanova, N.; Ghassemian, M.; Richardson, T. H.; Stege, J. T.; Cayouette, M.; McHardy, A. C.; Djordjevic, G.; Aboushadi, N., Metagenomic and functional analysis of hindgut microbiota of a wood-feeding higher termite. Nature 2007, 450 (7169), 560-565. 
22. Brune, A., Symbiotic digestion of lignocellulose in termite guts. Nature Reviews Microbiology 2014, 12, 168.

23. Keilich, G.; Bailey, P.; Liese, W., Enzymatic degradation of cellulose, cellulose derivatives and hemicelluloses in relation to the fungal decay of wood. Wood Science and Technology 1970, 4 (4), 273283.

24. Bhattacharjee, S. S.; Perlin, A. S., Enzymatic degradation of carboxymethylcellulose and other cellulose derivatives. Journal of Polymer Science Part C: Polymer Symposia 1971, 36 (1), 509-521.

25. Puls, J.; Wilson, S. A.; Hölter, D., Degradation of Cellulose Acetate-Based Materials: A Review. Journal of Polymers and the Environment 2011, 19 (1), 152-165.

26. Ooshima, H.; Burns, D.; O. Converse, A., Adsorption of cellulase from Trichoderma-Reesei on cellulose and lignacious residue in wood pretreated by dilute sulfuric-acid with explosive decompression. 1990; Vol. 36, p 446-452.

27. Sakai, K.; Yamauchi, T.; Nakasu, F.; Ohe, T., Biodegradation of Cellulose Acetate by Neisseria sicca. Bioscience, Biotechnology, and Biochemistry 1996, 60 (10), 1617-1622.

28. Plötze, M.; Niemz, P., Porosity and pore size distribution of different wood types as determined by mercury intrusion porosimetry. European Journal of Wood and Wood Products 2011, 69 (4), 649-657.

29. Ellerby, L. M.; Nishida, C. R., Encapsulation of proteins in transparent porous silicate glasses prepared by the sol-gel method. Science 1992, 255 (5048), 1113.

30. F. Gibbs, S. K., Inteaz Alli, Catherine N. Mulligan, Bernard, Encapsulation in the food industry: a review. International journal of food sciences and nutrition 1999, 50 (3), 213-224.

31. Gabizon, A.; Catane, R.; Uziely, B.; Kaufman, B.; Safra, T.; Cohen, R.; Martin, F.; Huang, A.; Barenholz, Y., Prolonged circulation time and enhanced accumulation in malignant exudates of doxorubicin encapsulated in polyethylene-glycol coated liposomes. Cancer research 1994, 54 (4), $987-$ 992.

32. Iqbal, M.; Zafar, N.; Fessi, H.; Elaissari, A., Double emulsion solvent evaporation techniques used for drug encapsulation. International Journal of Pharmaceutics 2015, 496 (2), 173-190.

33. Yahaya khan, M.; Abdul Karim, Z. A.; Hagos, F.; Rashid A Aziz, A.; Tan, I., Current Trends in Water-in-Diesel Emulsion as a Fuel. 2014; Vol. 2014, p 527472.

34. Wittbecker, E. L.; Morgan, P. W., Interfacial polycondensation. I. Journal of Polymer Science 1959, 40 (137), 289-297.

35. Raaijmakers, M. J. T.; Benes, N. E., Current trends in interfacial polymerization chemistry. Progress in Polymer Science 2016, 63 (Supplement C), 86-142.

36. Latnikova, A.; Yildirim, A., Thermally induced release from polymeric microparticles with liquid core: the mechanism. Soft Matter 2015, 11 (10), 2008-2017.

37. Scher, H. B.; Rodson, M.; Lee, K. S., Microencapsulation of pesticides by interfacial polymerization utilizing isocyanate or aminoplast chemistry. Pest Management Science 1998, 54 (4), 394-400.

38. Florence, A. T.; Whitehill, D., Some features of breakdown in water-in-oil-in-water multiple emulsions. Journal of Colloid and Interface Science 1981, 79 (1), 243-256.

39. Morais, J. M.; Santos, O. D. H.; Friberg, S. E., Some Fundamentals of the One-Step Formation of Double Emulsions. Journal of Dispersion Science and Technology 2010, 31 (8), 1019-1026.

40. Jiao, J.; Rhodes, D. G.; Burgess, D. J., Multiple emulsion stability: pressure balance and interfacial film strength. Journal of colloid and interface science 2002, 250 (2), 444-450.

41. Sappidi, S.; Thadkala, K.; Kota, J.; Aukunuru, J., Preparation and Characterization of Ethyl Cellulose Microspheres Encapsulating Metformin Hydrochloride and Glipizide. Schol. Res. Lib 2014, 6 (4), 213-226.

42. Whitesides, G. M., The origins and the future of microfluidics. Nature 2006, 442 (7101), 368-

373.

43. https://courses.lumenlearning.com/chemistryformajors/chapter/properties-of-liquids/. 
44. Zhao, C.-X., Multiphase flow microfluidics for the production of single or multiple emulsions for drug delivery. Advanced Drug Delivery Reviews 2013, 65 (11-12), 1420-1446.

45. Liu, L.; Yang, J.-P.; Ju, X.-J.; Xie, R.; Yang, L.; Liang, B.; Chu, L.-Y., Microfluidic preparation of monodisperse ethyl cellulose hollow microcapsules with non-toxic solvent. Journal of Colloid and Interface Science 2009, 336 (1), 100-106.

46. Chu, L. Y.; Utada, A. S.; Shah, R. K.; Kim, J. W.; Weitz, D. A., Controllable monodisperse multiple emulsions. Angewandte Chemie International Edition 2007, 46 (47), 8970-8974.

47. Vitale, S. A.; Katz, J. L., Liquid Droplet Dispersions Formed by Homogeneous Liquid-Liquid Nucleation: "The Ouzo Effect". Langmuir 2003, 19 (10), 4105-4110.

48. Hornig, S.; Heinze, T., Efficient Approach To Design Stable Water-Dispersible Nanoparticles of Hydrophobic Cellulose Esters. Biomacromolecules 2008, 9 (5), 1487-1492.

49. Wondraczek, H.; Petzold-Welcke, K.; Fardim, P.; Heinze, T., Nanoparticles from conventional cellulose esters: evaluation of preparation methods. Cellulose 2013, 20 (2), 751-760.

50. Shekunov, B. Y.; York, P., Crystallization processes in pharmaceutical technology and drug delivery design. Journal of Crystal Growth 2000, 211 (1-4), 122-136.

51. Lepeltier, E.; Bourgaux, C.; Couvreur, P., Nanoprecipitation and the "Ouzo effect": Application to drug delivery devices. Advanced Drug Delivery Reviews 2014, 71, 86-97.

52. Perazzo, A.; Preziosi, V.; Guido, S., Phase inversion emulsification: Current understanding and applications. Advances in Colloid and Interface Science 2015, 222, 581-599.

53. Cao, Y.; You, B.; Wu, L., Facile Fabrication of Hollow Polymer Microspheres through the PhaseInversion Method. Langmuir 2010, 26 (9), 6115-6118.

54. Fernandez, P.; André, V.; Rieger, J.; Kühnle, A., Nano-emulsion formation by emulsion phase inversion. Colloids and Surfaces A: Physicochemical and Engineering Aspects 2004, 251 (1), 53-58.

55. Komaiko, J.; McClements, D., Formation of Food-Grade Nanoemulsions Using Low-Energy Preparation Methods: A Review of Available Methods. 2016; Vol. 15, p n/a-n/a.

56. Sajjadi, S., Nanoemulsion Formation by Phase Inversion Emulsification: On the Nature of Inversion. Langmuir 2006, 22 (13), 5597-5603.

57. Jahanzad, F.; Josephides, D.; Mansourian, A.; Sajjadi, S., Dynamics of Transitional Phase Inversion Emulsification: Effect of Addition Time on the Type of Inversion and Drop Size. Industrial \& Engineering Chemistry Research 2010, 49 (16), 7631-7637.

58. Duong-Ly, K. C.; Gabelli, S. B., Chapter Seven - Salting out of Proteins Using Ammonium Sulfate Precipitation. In Methods in Enzymology, Lorsch, J., Ed. Academic Press: 2014; Vol. 541, pp 85-94.

59. Fonseca, A.; Ferreira, P.; Cordeiro, R.; Mendonça, P.; Góis, J.; Gil, H.; Coelho, J., Drug Delivery Systems for Predictive Medicine: Polymers as Tools for Advanced Applications. 2013.

60. Perugini, P.; Simeoni, S.; Scalia, S.; Genta, I.; Modena, T.; Conti, B.; Pavanetto, F., Effect of nanoparticle encapsulation on the photostability of the sunscreen agent, 2-ethylhexyl-pmethoxycinnamate. International Journal of Pharmaceutics 2002, 246 (1-2), 37-45.

61. Schultz, T. P.; Nicholas, D. D.; Preston, A. F., A brief review of the past, present and future of wood preservation. Pest management science 2007, 63 (8), 784-788.

62. Colley, R. H., The Evaluation of Wood Preservatives Part I. Bell Labs Technical Journal 1953, 32 (1), 120-169.

63. Weis, P.; Weis, J. S.; Greenberg, A.; Nosker, T. J., Toxicity of construction materials in the marine environment: A comparison of chromated-copper-arsenate-treated wood and recycled plastic. Archives of Environmental Contamination and Toxicology 1992, 22 (1), 99-106.

64. Hirata, T.; Inoue, M.; Fukui, Y., Pyrolysis and combustion toxicity of wood treated with CCA. Wood Science and Technology 1992, 27 (1), 35-47.

65. Brown, C. J.; Eaton, R. A., Toxicity of Chromated Copper Arsenate (CCA)-Treated Wood to NonTarget Marine Fouling Communities in Langstone Harbour, Portsmouth, UK. Marine Pollution Bulletin 2001, 42 (4), 310-318.

66. Hingston, J. A.; Collins, C. D.; Murphy, R. J.; Lester, J. N., Leaching of chromated copper arsenate wood preservatives: a review. Environmental Pollution 2001, 111 (1), 53-66. 
67. Grace, J. K.; Byrne, A.; Morris, P. I.; Tsunoda, K., Performance of borate-treated lumber after 8 years in an above-ground termite field test in Hawaii. International Research Group on Wood Preservation. Stockholm, Sweden. IRG/WP 2006, 06-30390.

68. Obanda, D. N.; Shupe, T. F.; Barnes, H. M., Reducing leaching of boron-based wood preservatives - A review of research. Bioresource Technology 2008, 99 (15), 7312-7322.

69. Association, A. W. P., AWPA book of standards. American Wood Protection Association: 2008. 70. Limited, S. A. I., AS 1604.1: 2012. Specification for Preservative Treatment. Part 1: Sawn and Round Timber. Standards Australia: 2012.

71. Tyagi, V.; Kaushik, S. C.; Tyagi, S.; Akiyama, T., Renewable and Sustainable Energy Reviews. 2018.

72. Heinze, T.; Liebert, T., Unconventional methods in cellulose functionalization. Progress in Polymer Science 2001, 26 (9), 1689-1762.

73. Ren, B.; Cai, L.; Zhang, L.-R.; Yang, Z.-J.; Zhang, L.-H., Selective deacetylation using iodinemethanol reagent in fully acetylated nucleosides. Tetrahedron letters 2005, 46 (47), 8083-8086.

74. Gomez-Bujedo, S.; Fleury, E.; Vignon, M. R., Preparation of cellouronic acids and partially acetylated cellouronic acids by TEMPO/ $\mathrm{NaClO}$ oxidation of water-soluble cellulose acetate. Biomacromolecules 2004, 5 (2), 565-571.

75. Cho, J.-S.; Kwon, A.; Cho, C.-G., Microencapsulation of octadecane as a phase-change material by interfacial polymerization in an emulsion system. Colloid and polymer science 2002, 280 (3), 260 266.

76. Taylor, P., Ostwald ripening in emulsions. Advances in Colloid and Interface Science 1998, 75 (2), 107-163.

77. Shakesheff, K. M.; Evora, C.; Soriano, I.; Langer, R., The Adsorption of Poly(vinyl alcohol) to Biodegradable Microparticles Studied by X-Ray Photoelectron Spectroscopy (XPS). Journal of Colloid and Interface Science 1997, 185 (2), 538-547.

78. Schulze, P.; Gericke, M.; Scholz, F.; Wondraczek, H.; Miethe, P.; Heinze, T., Incorporation of Hydrophobic Dyes within Cellulose Acetate and Acetate Phthalate Based Nanoparticles. Macromolecular Chemistry and Physics 2016, 217 (16), 1823-1833.

79. Mohamed, A., FORMULATION, CHARACTERIZATION AND IN VIVO APPLICATION OF ORAL INSULIN NANOTECHNOLOGY USING DIFFERENT BIODEGRADABLE POLYMERS: ADVANCED DRUG DELIVERY SYSTEM. 2018.

80. Di Renzo, F.; Bacchetta, R.; Bizzo, A.; Giavini, E.; Menegola, E., Is the amphibian X. laevis WEC a good alternative method to rodent WEC teratogenicity assay? The example of the three triazole derivative fungicides Triadimefon, Tebuconazole, Cyproconazole. Reproductive Toxicology 2011, 32 (2), 220-226.

\section{Appendix}

\subsection{Dynamic light scattering (DLS) graphs}

The DLS graphs shown here are representative of the particle formulations generated in the project for reference. 


\subsubsection{Zetasizer}

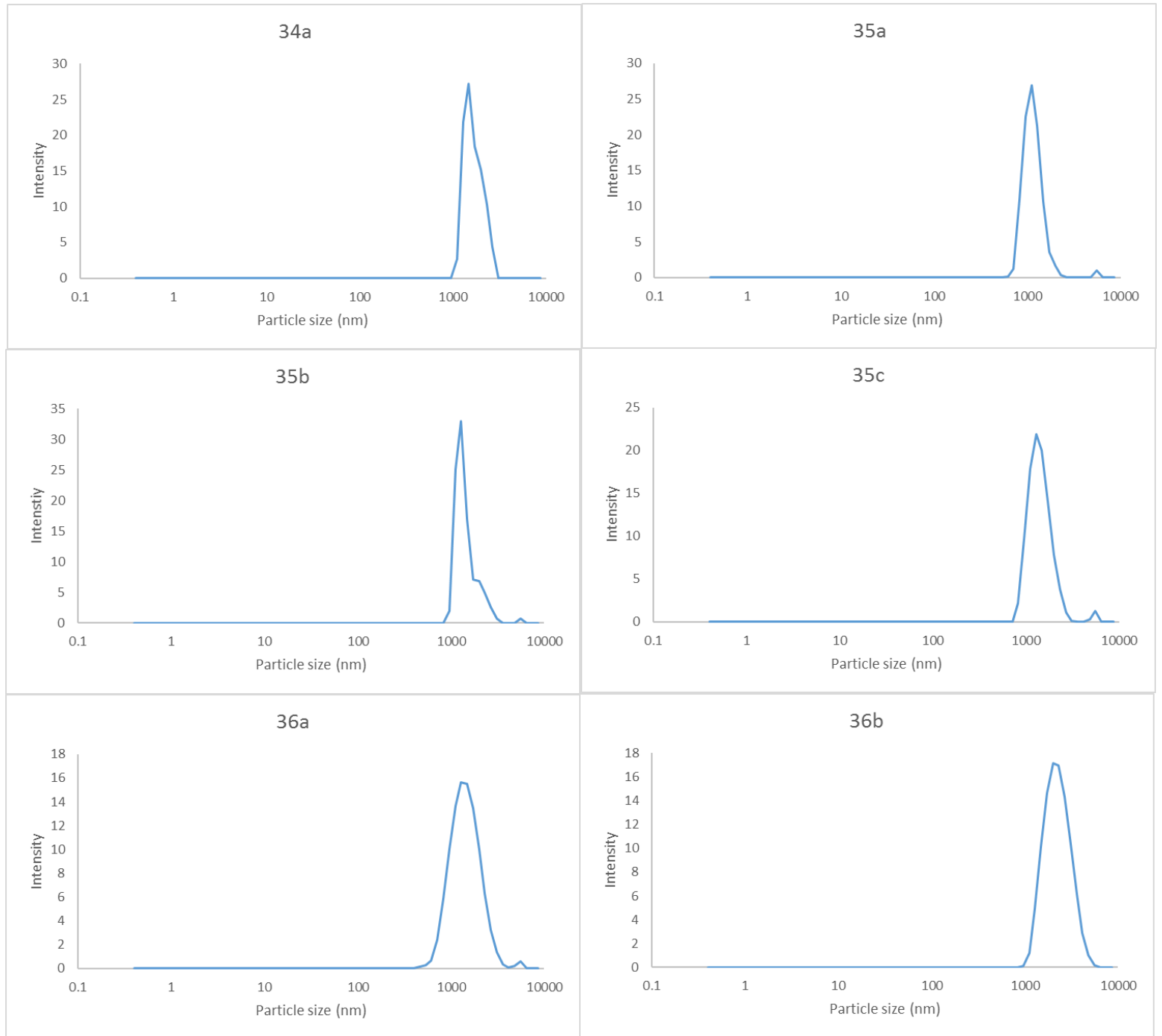




\subsubsection{Mastersizer 3000}
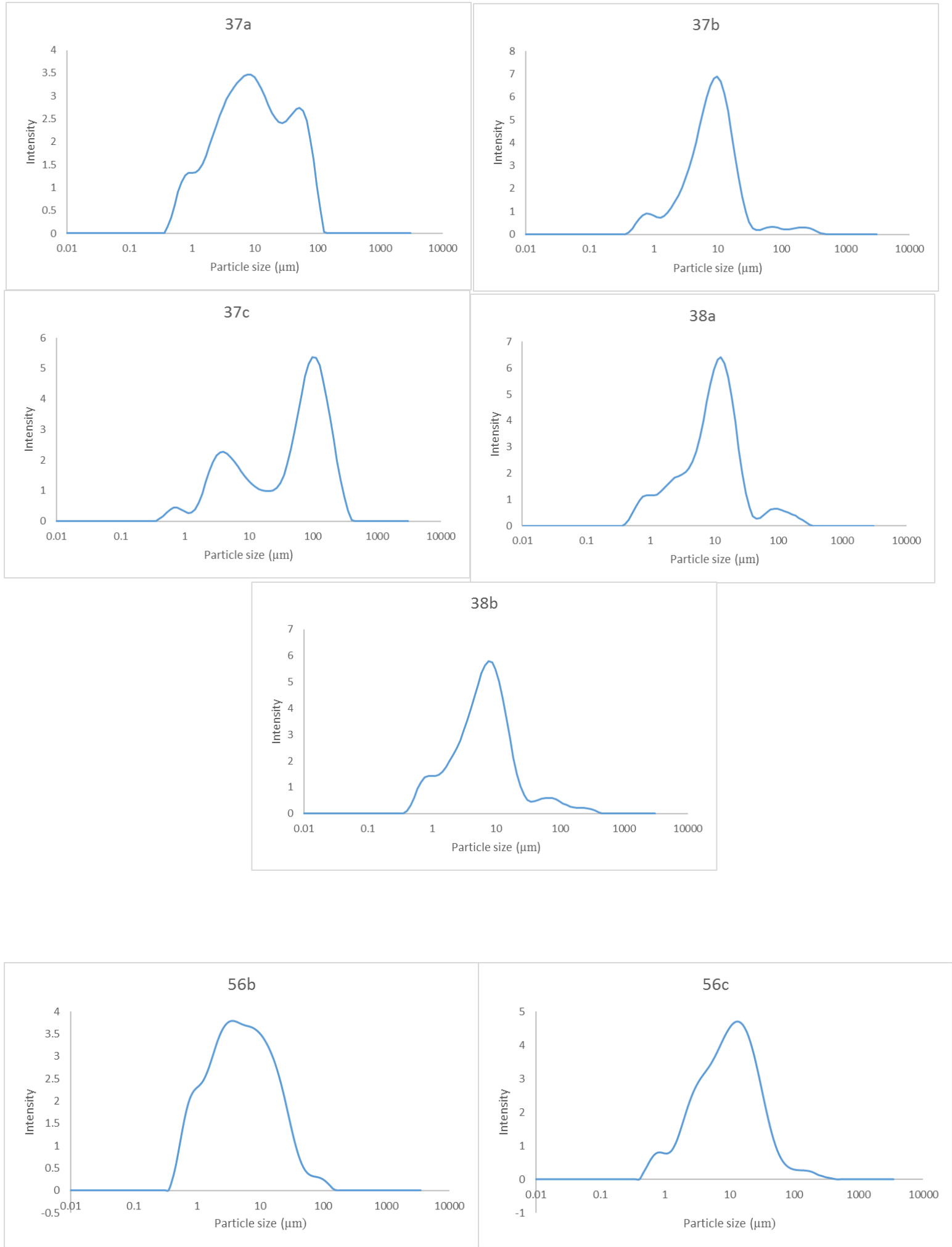

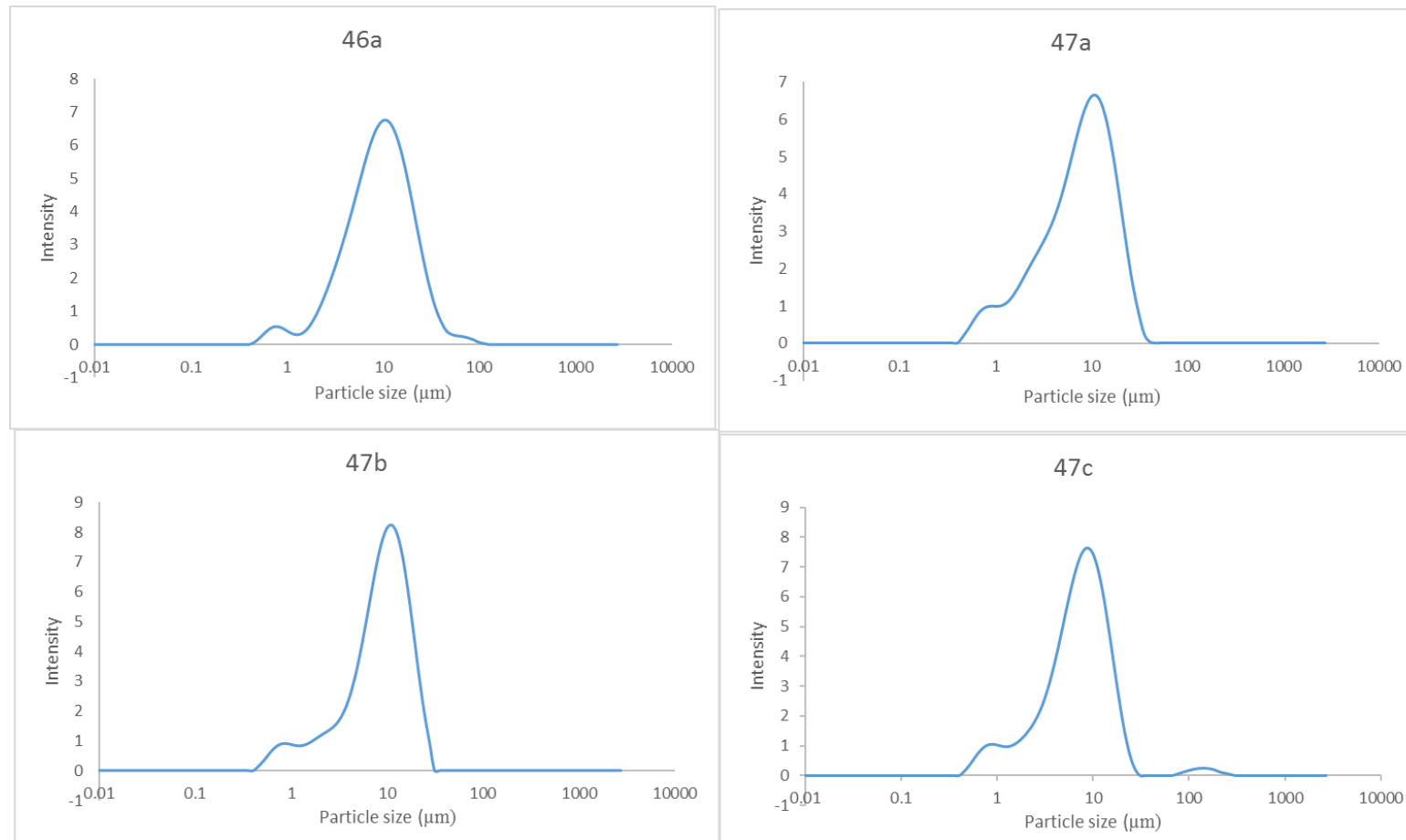

$47 c$
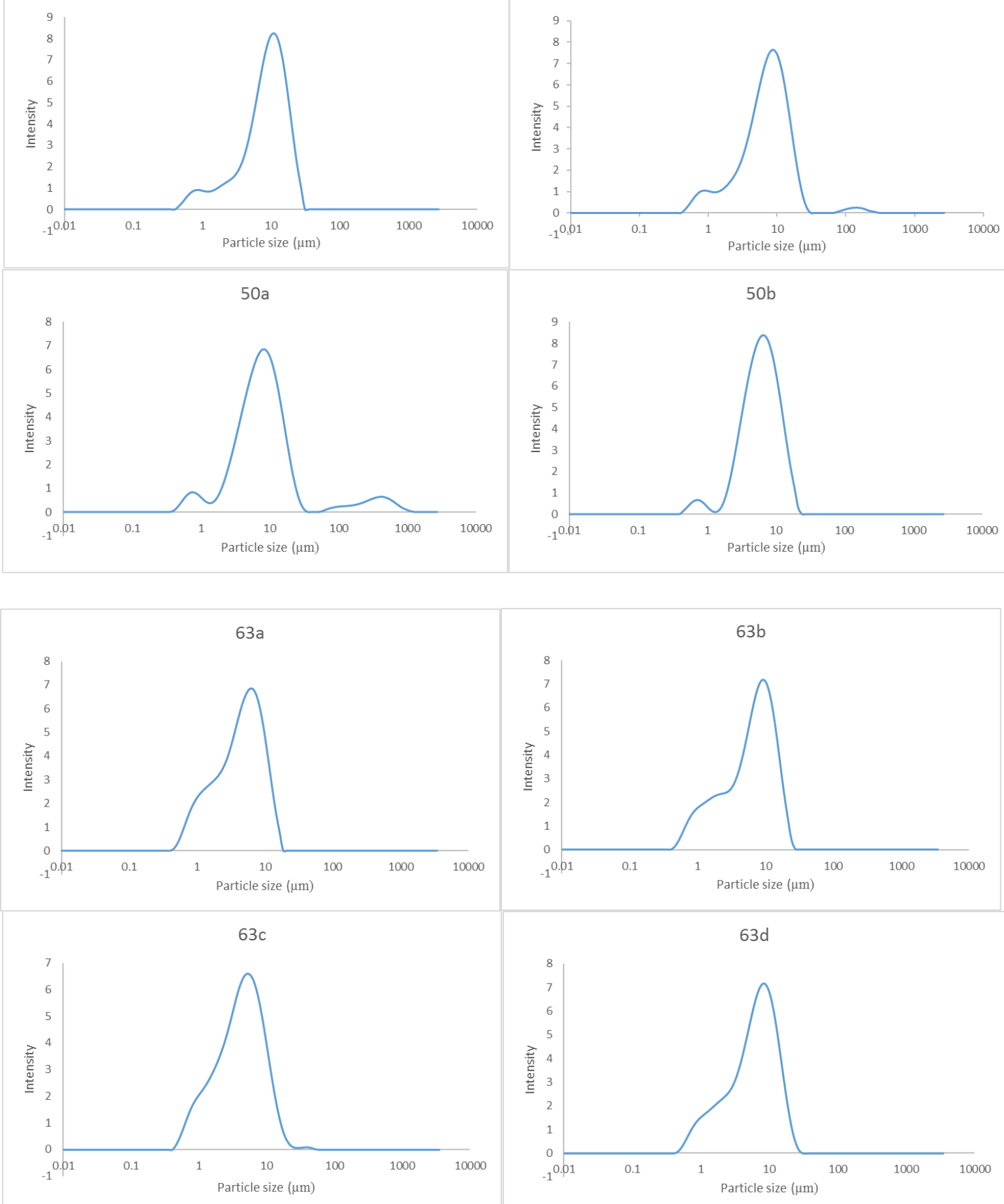


\section{$6.2{ }^{1} \mathrm{H}$ nuclear magnetic resonance (NMR) spectra}

The ${ }^{1} \mathrm{H}$ NMR spectra shown here are for reference in the elucidation of particle composition of the particle formulation generated by phase inversion in this project.

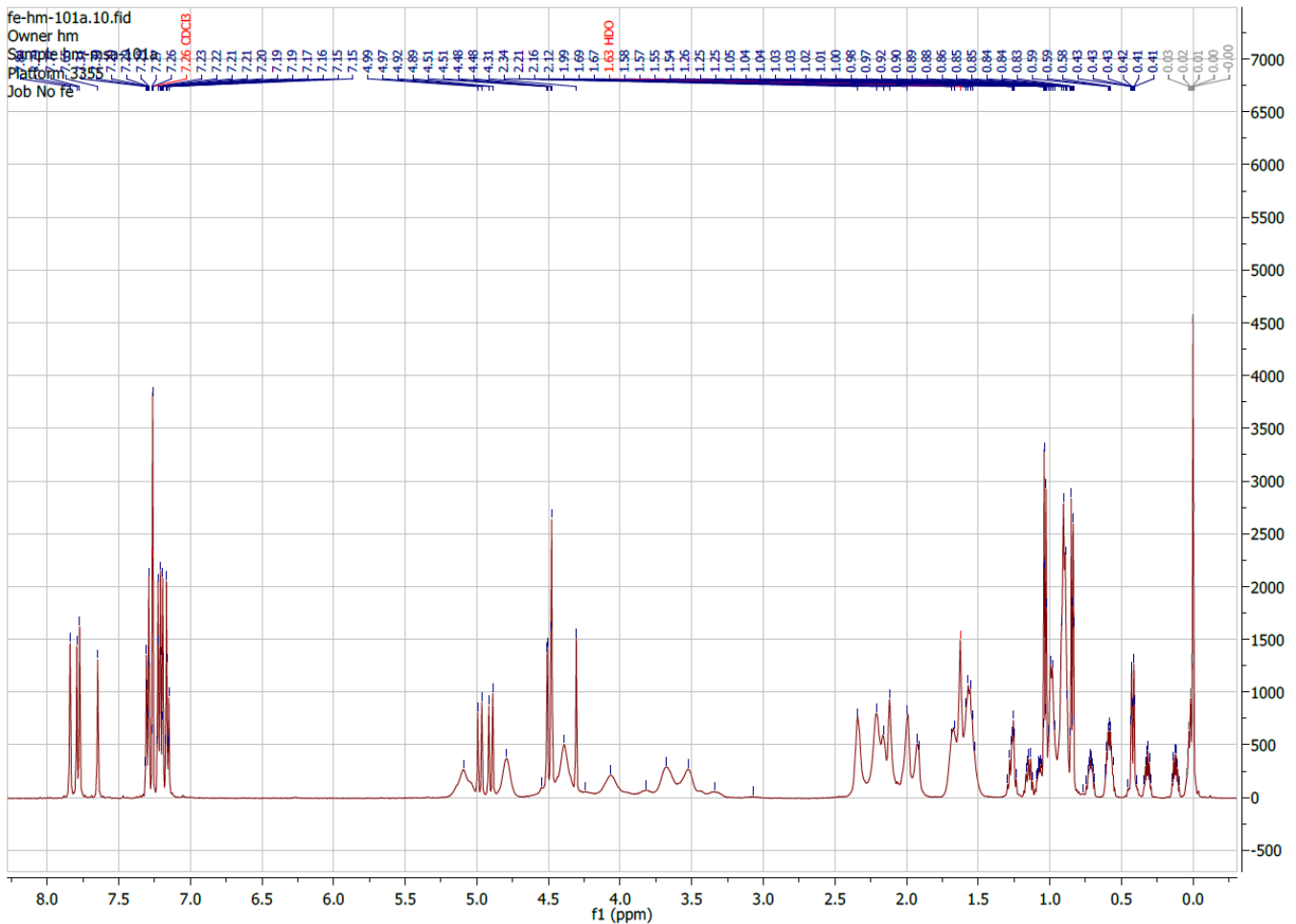

Sample 101a

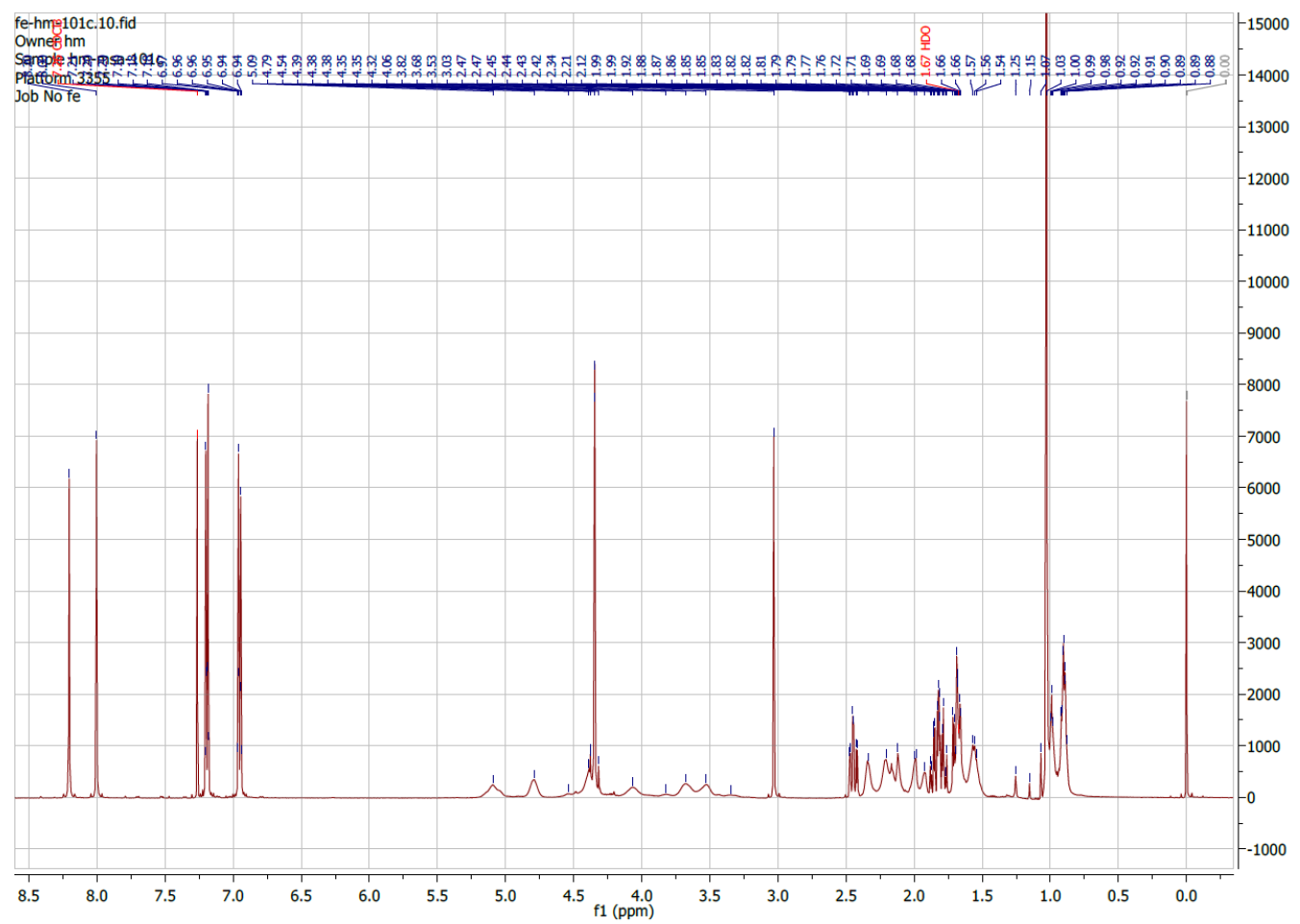

Sample 101c 


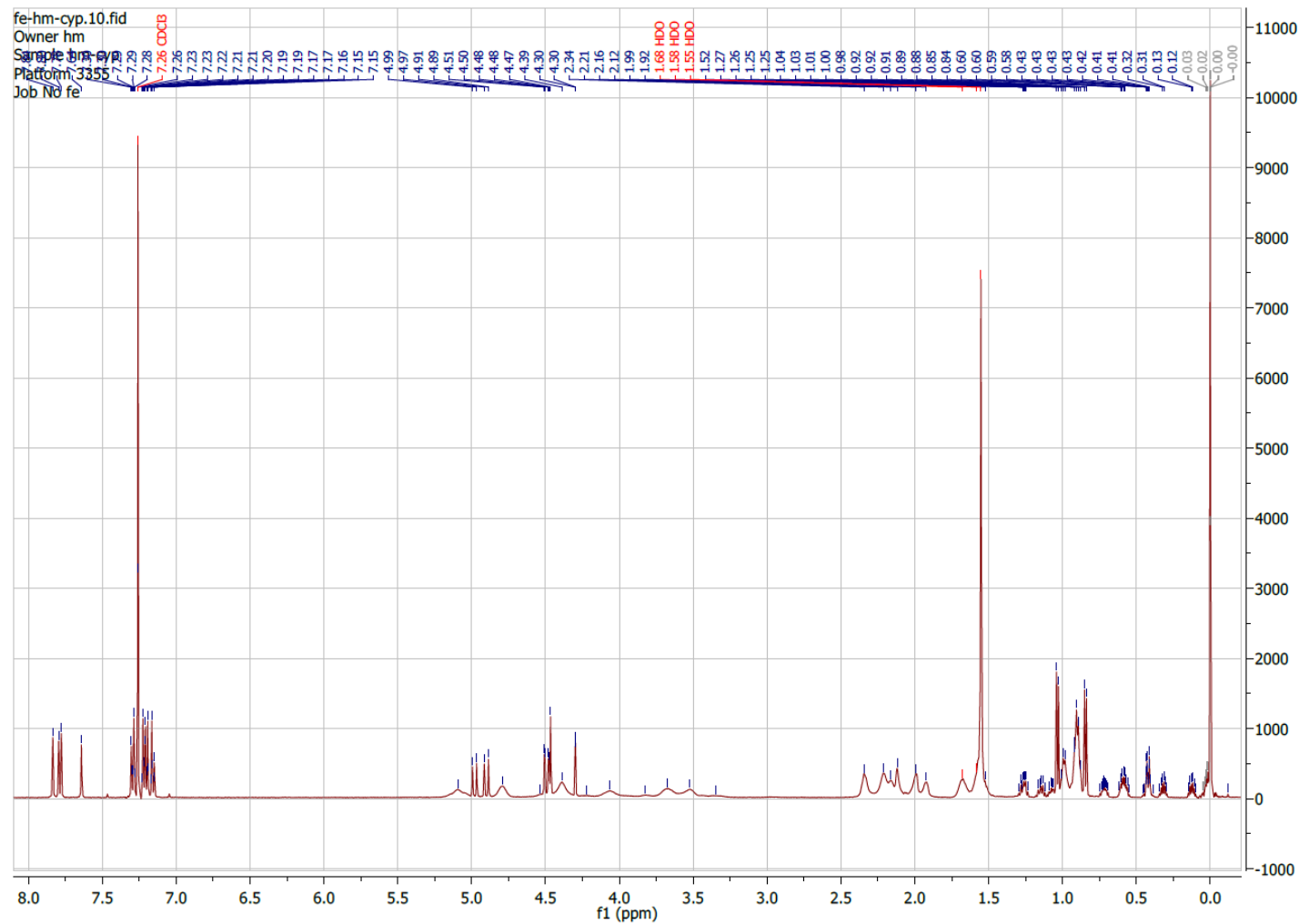

\section{Cyproconazole}

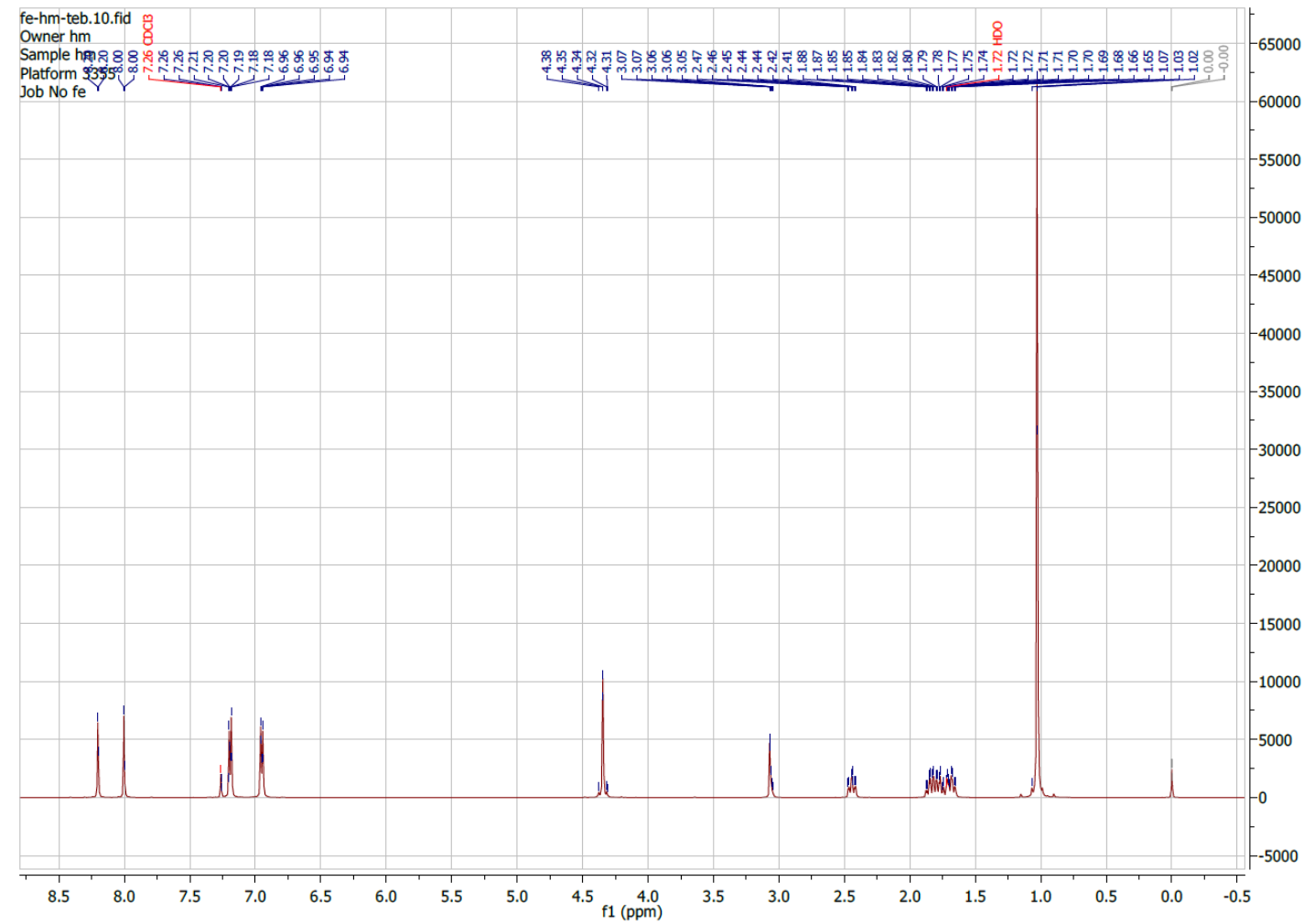

Tebuconazole 


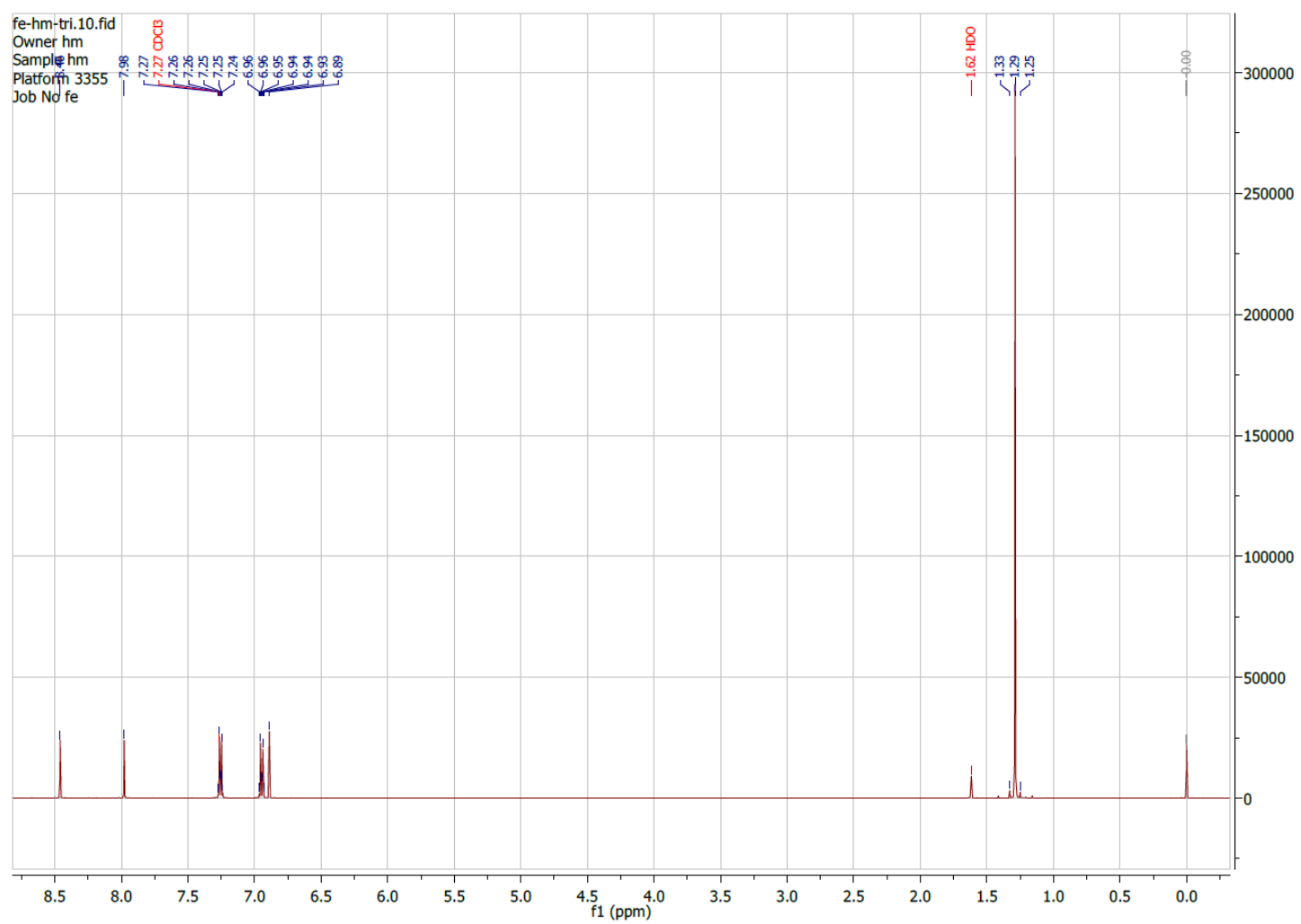

Triadimefon

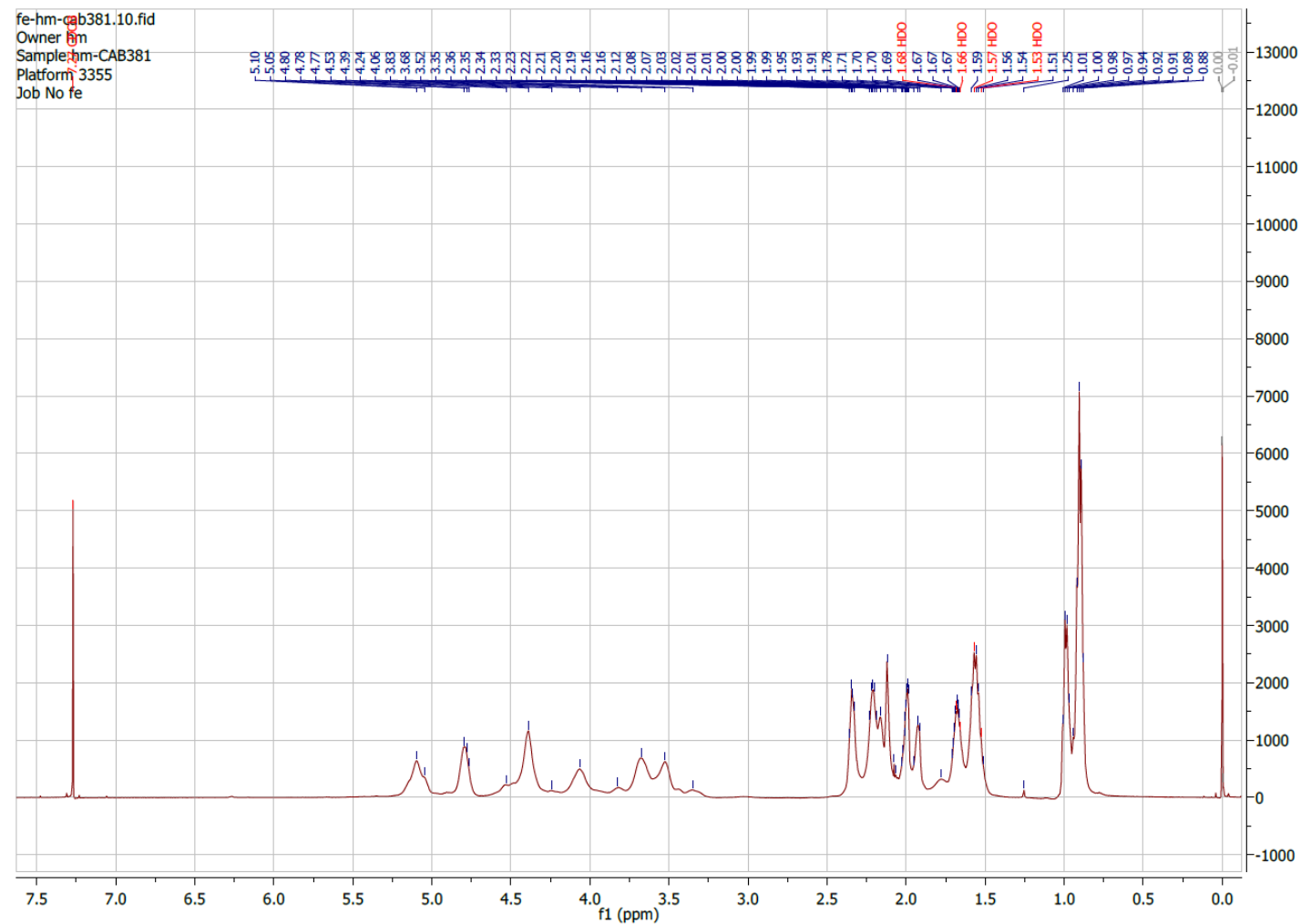

CAB 381.01 


\subsection{Infrared spectroscopy (IR)}

The IR spectra shown here are for reference to the model compound incorporation that was done to test as a method of detection in the particles generated by phase inversion.

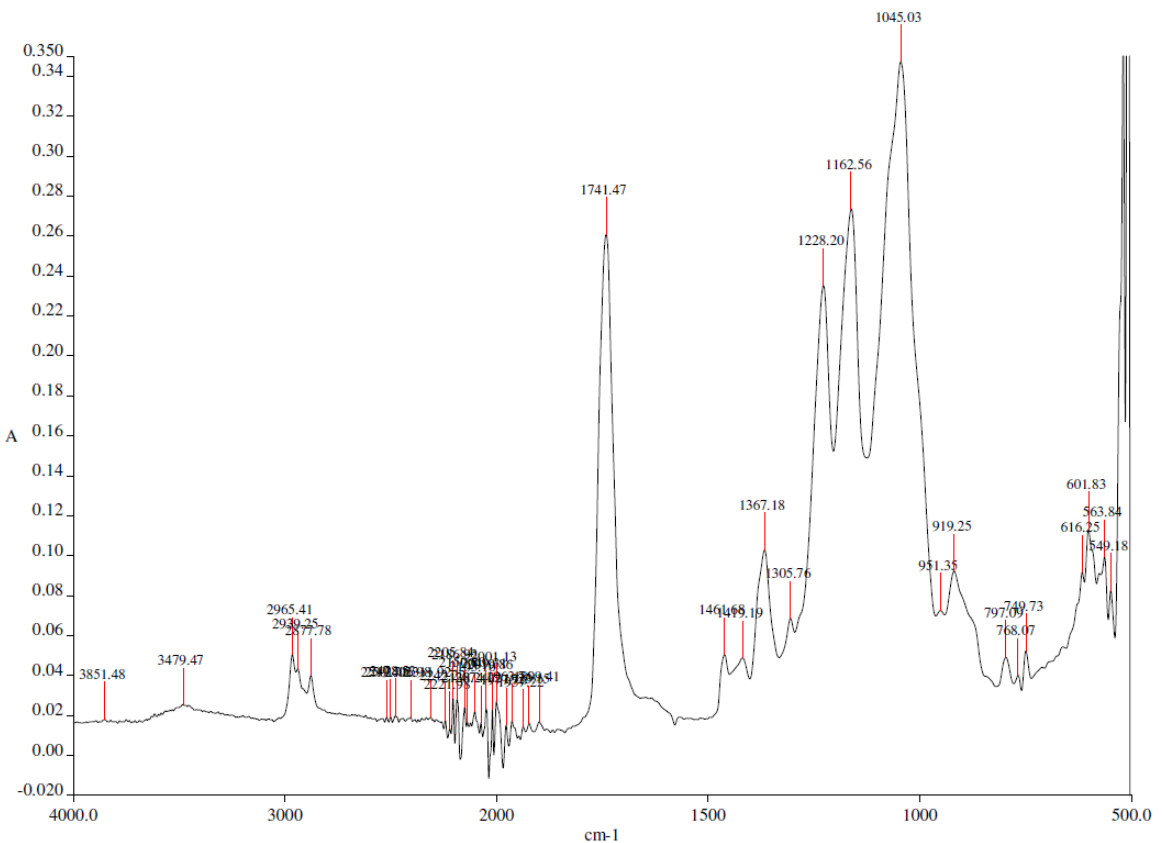

Sample 47a

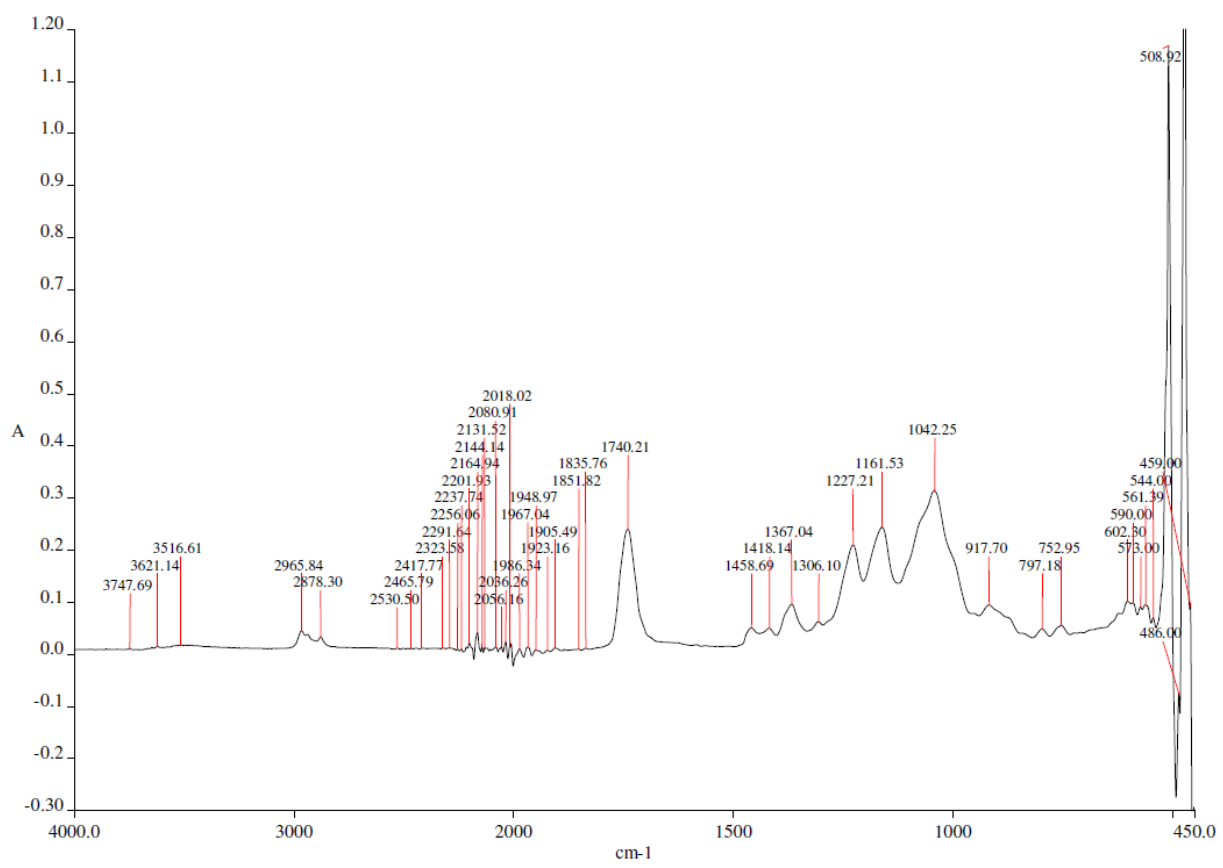

Sample 47b 


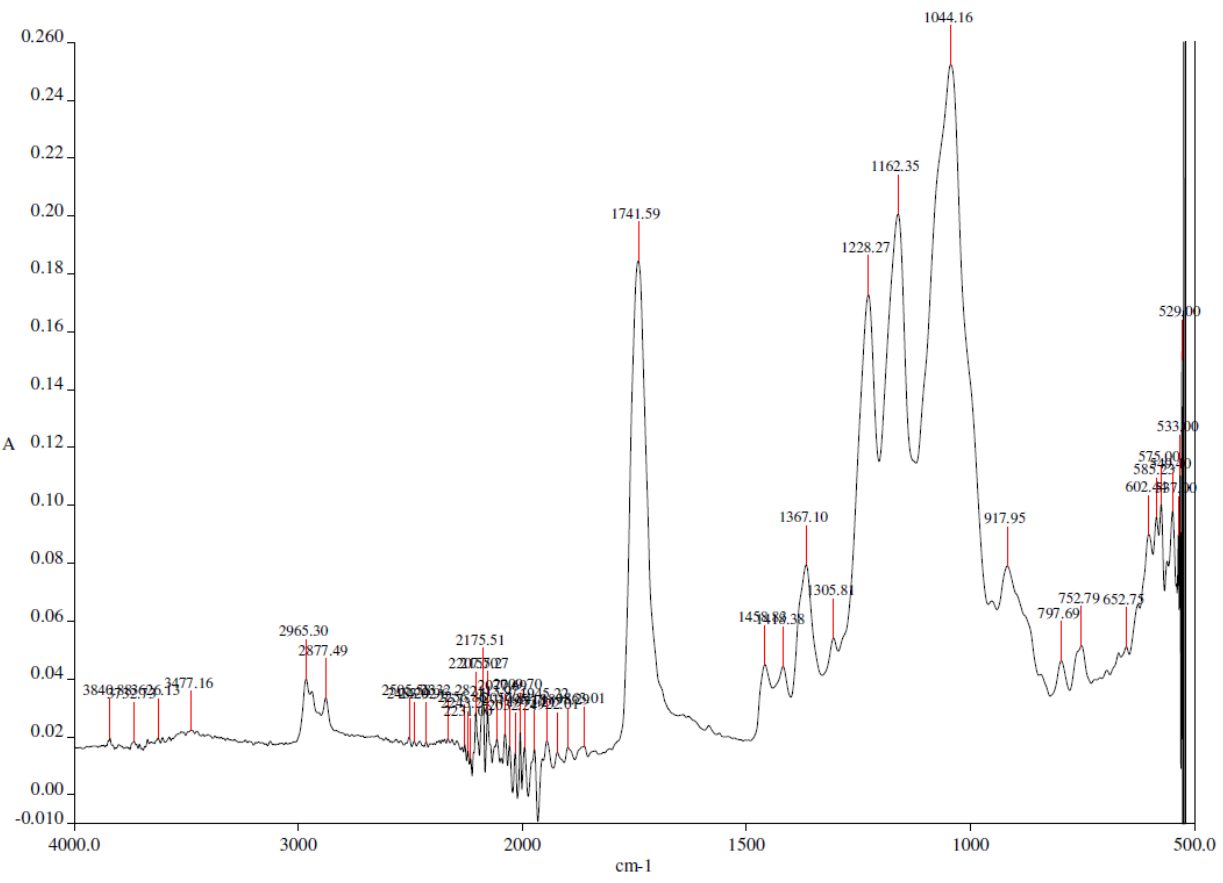

Sample 47c

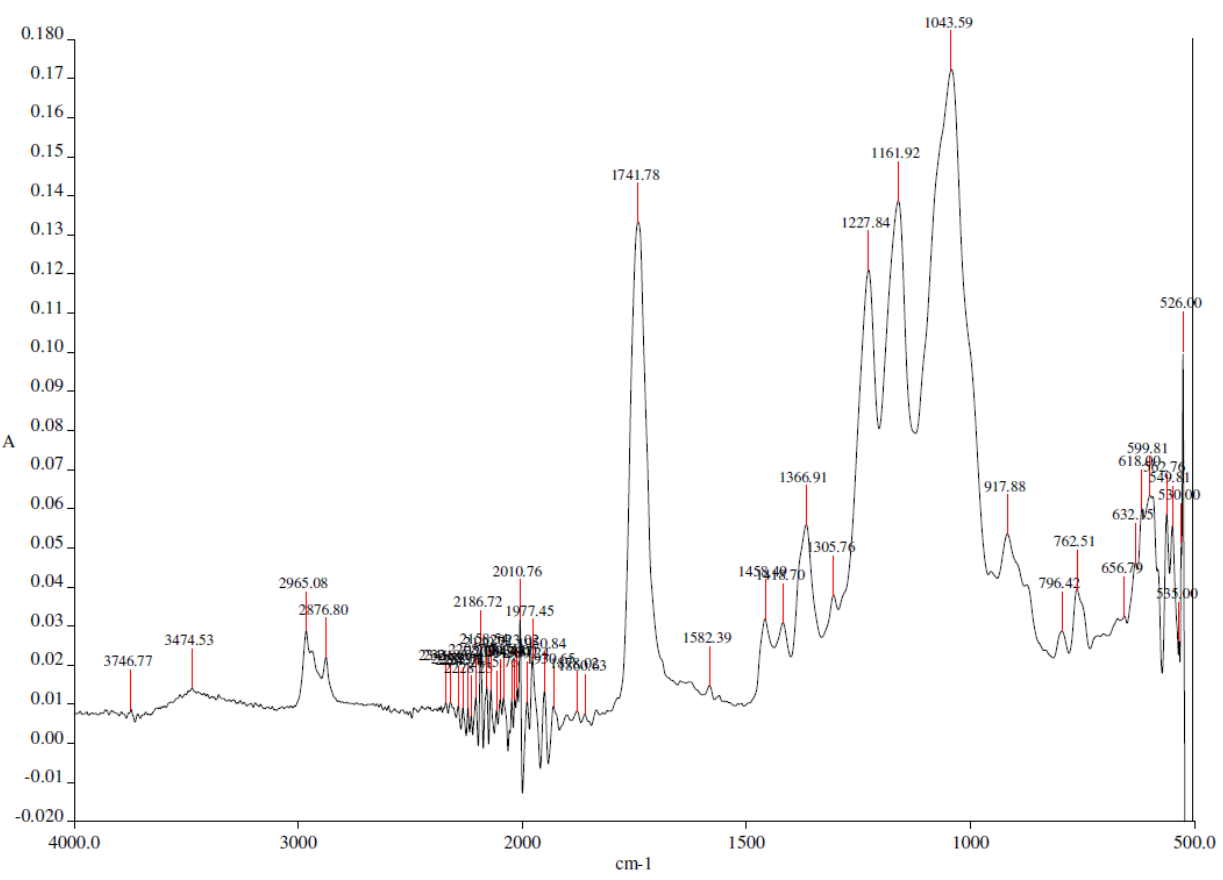

Sample 50a 


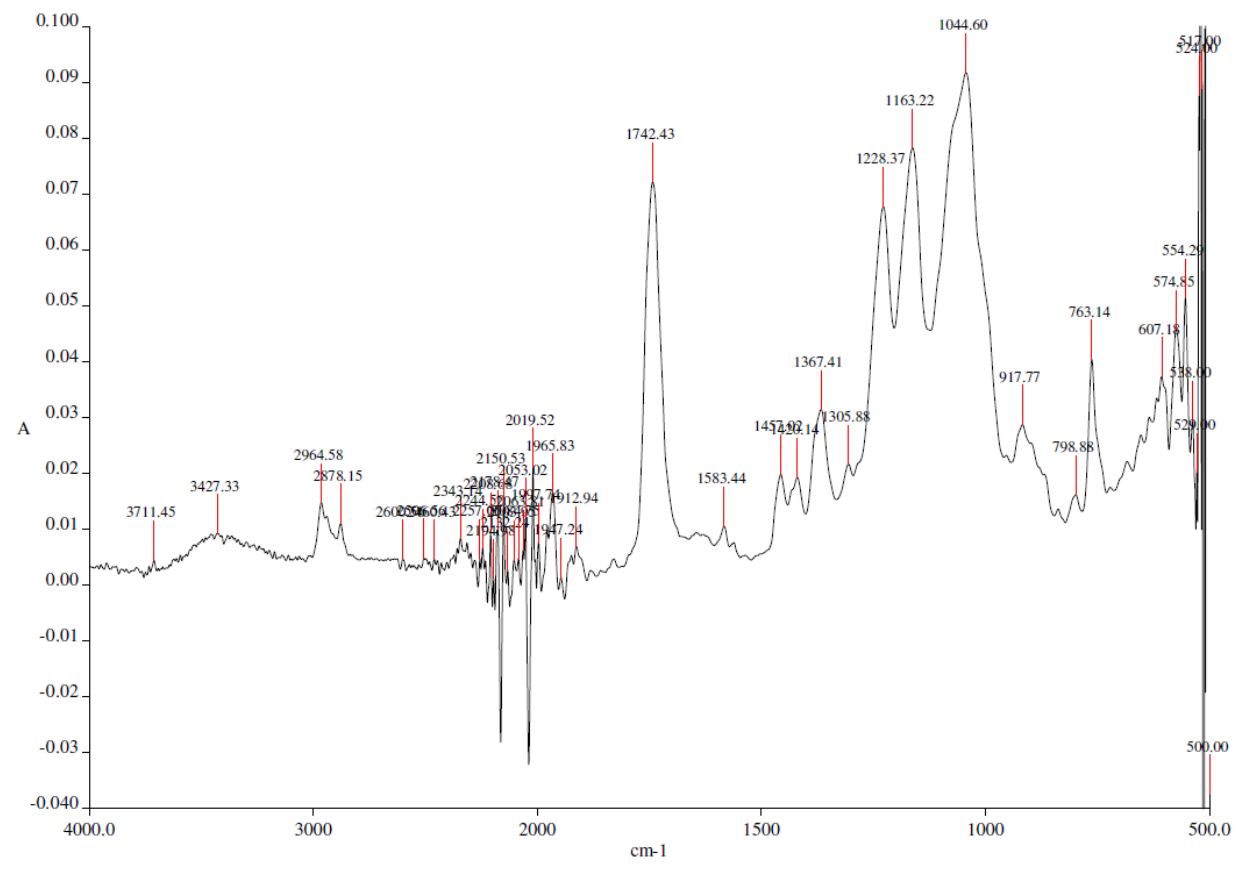

Sample 50b

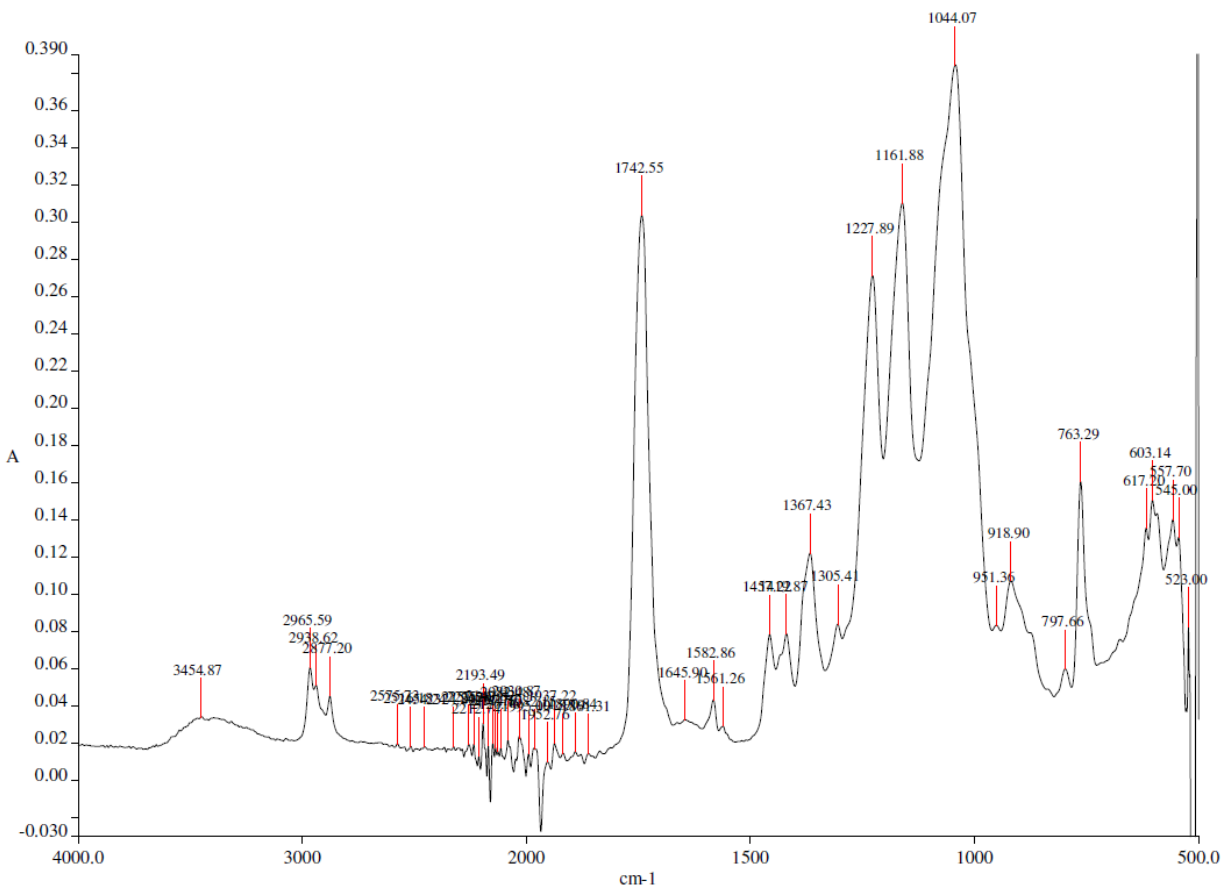

Sample 50c 


\subsection{Dynamic scanning calorimetry (DSC) graphs}

The DSC graphs are for reference for particle formulations generated by interfacial polymerisation with cellulosic derivatives as additives.

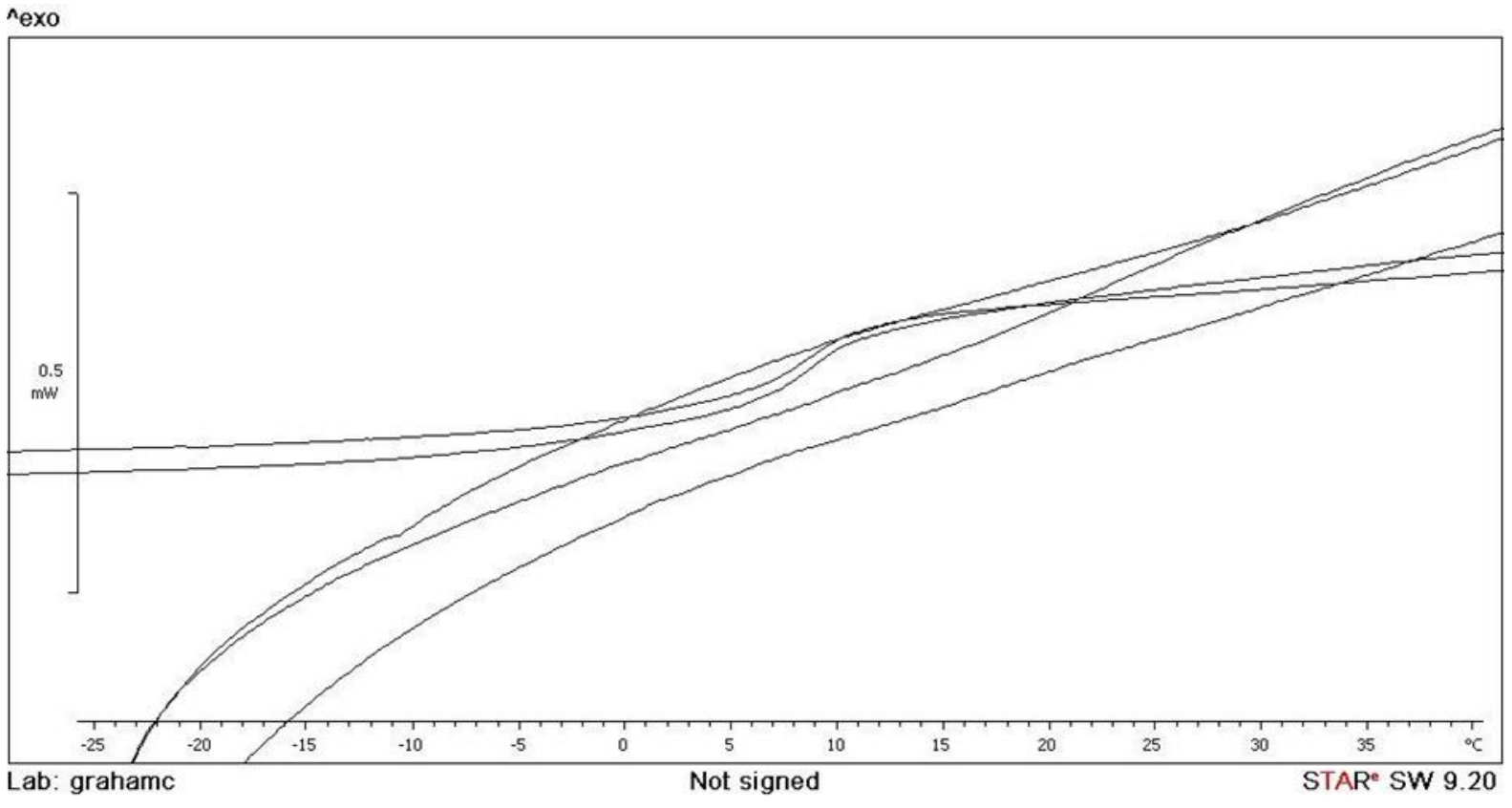

\section{Sample 12a}

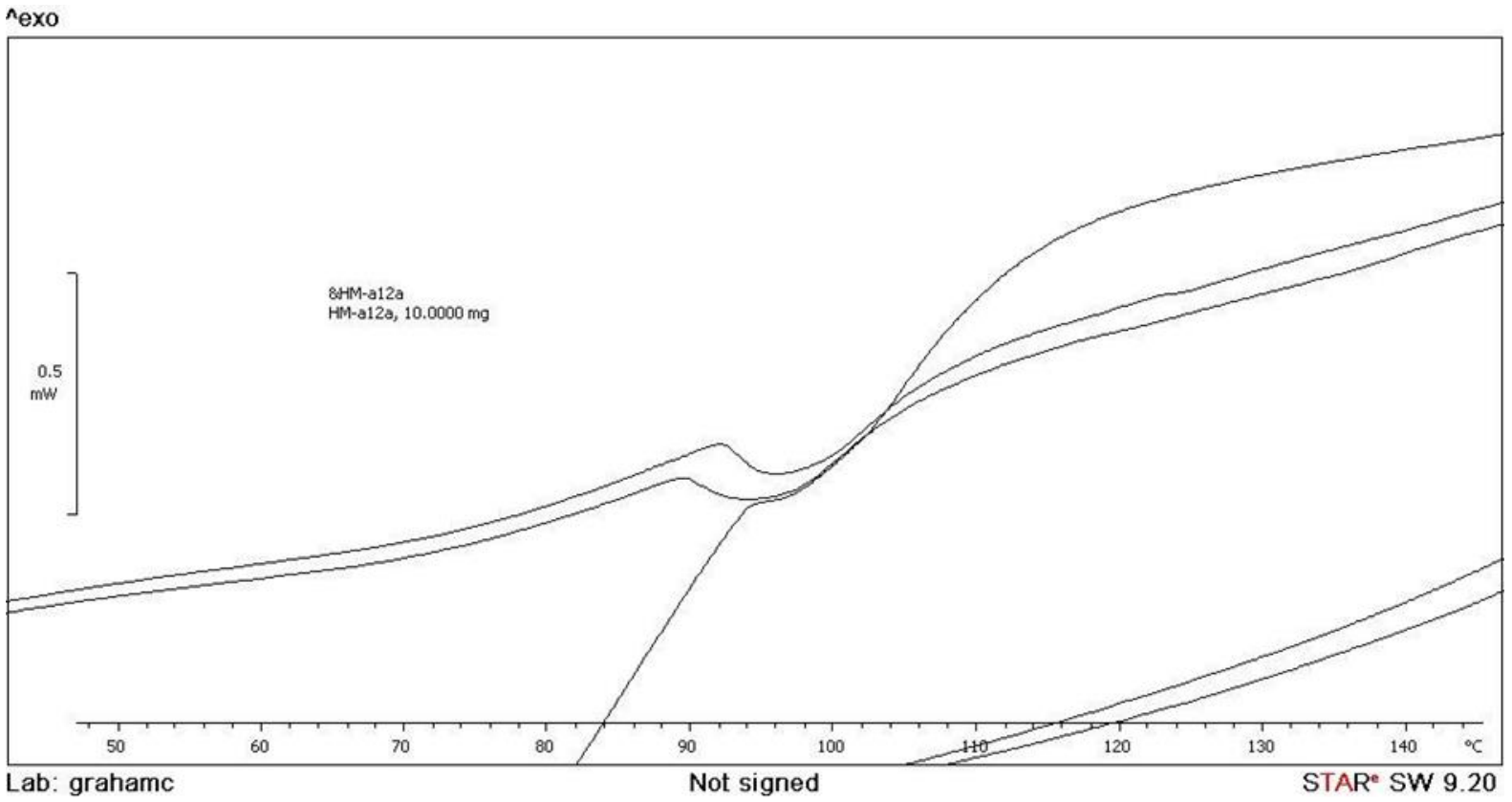

Sample 18a 